\title{
COMPORTAMIENTO SÍSMICO DE ESTRUCTURAS PORTICADAS DE HORMIGÓN ARMADO REACONDICIONADAS SÍSMICAMENTE CON MUROS DE FÁBRICA DE LADRILLO
}
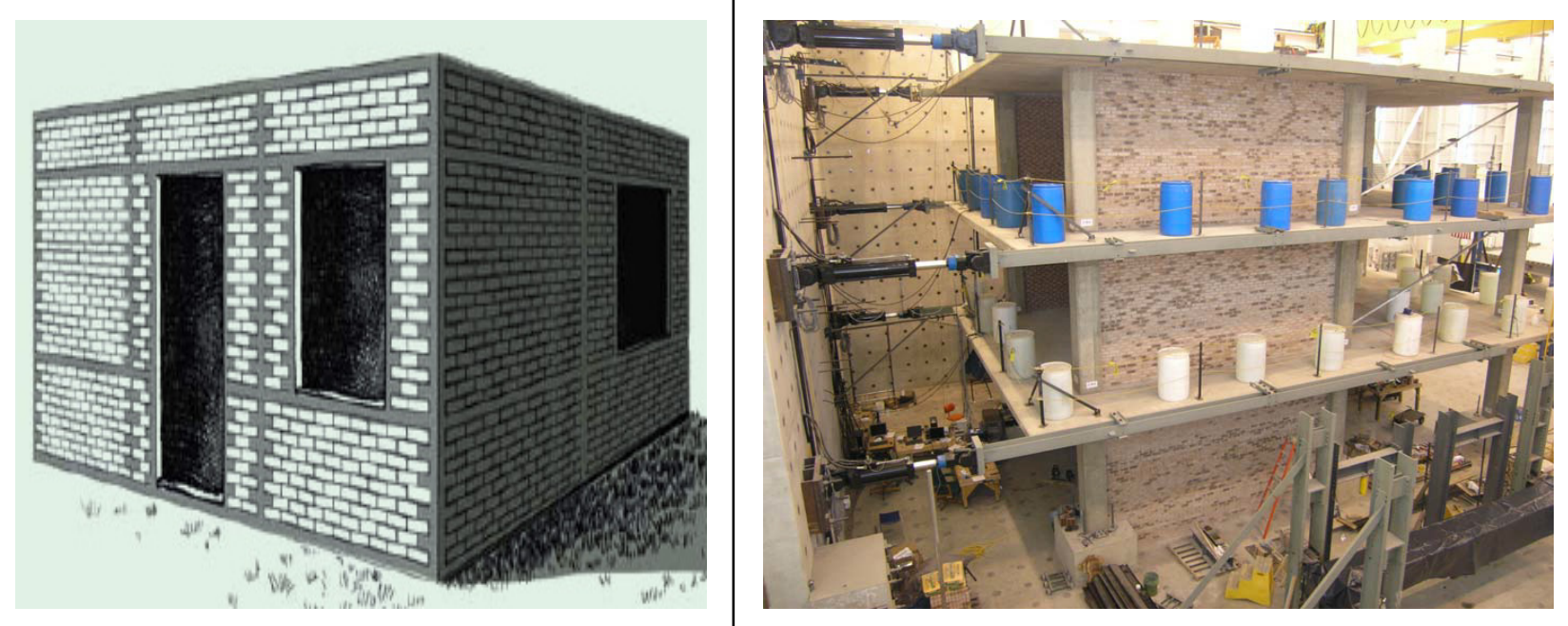

TRABAJO FIN DE MÁSTER MÁSTER DE ESTRUCTURAS

AlumNa: ANA LUISA RAMÍREZ MÁRQUEZ

TUTOR: AMADEO BENAVENT-CLIMENT

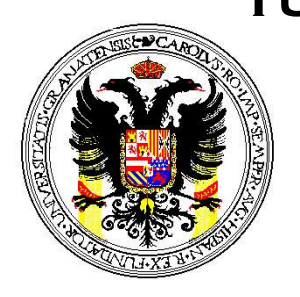

UNIVERSIDAD DE GRANADA

DEPARTAMENTO DE MECÁNICA DE ESTRUCTURAS E INGENIERÍA HIDRÁULICA 


\section{INDICE}

1. OBJETIVOS Y METODOLOGÍA EMPLEADA

2. ANTECEDENTES. BIBLIOGRAFÍA. ESTADO DEL ARTE 8

3. DEFINICIÓN DE PROTOTIPOS DE ESCUELAS 13

4. DIMENSIONADO DE PROTOTIPOS EN TRICALC 17

5. DESARROLLO DE MODELOS NUMERICOS DE LOS PROTOTIPOS PARA SU ANÁLSIS DINÁMICO CON EL PROGRAMA IDARC $\quad 19$

6. SELECCIÓN DE ACELEROGRAMAS Y CRITERIO DE ESCALADO 34

7. CONFIGURACIONES DE MUROS EN LOS PÓRTICOS 42

8. CARACTERIZACIÓN DEL DAÑO EN LA ESTRUCTURA Y DEL AREA DE MUROS. RESULTADOS DE LOS CÁLCULOS DINÁMICOS DIRECTOS 65

9. CONCLUSIONES 70

10. LIMITACIONES DE LA INVESTIGACIÓN Y TRABAJO FUTURO 76

$\begin{array}{ll}\text { BIBLIOGRAFÍA } & 77\end{array}$

$\begin{array}{ll}\text { ANEXO 1: Planos de Estructura } & 79\end{array}$ 


\section{OBJETIVOS Y METODOLOGÍA EMPLEADA}

\section{Introducción y Objetivos}

Los muros de fábrica de ladrillo se emplean en todo el mundo formando parte de cerramientos exteriores o como particiones interiores. Estudios de campo e inspecciones de estructuras que han sufrido terremotos severos en el pasado han puesto de manifiesto que en determinadas condiciones (continuidad, ausencia de aberturas etc.), estos muros (normalmente considerados elementos no estructurales) pueden ayudar a reducir la vulnerabilidad de las estructuras. Hay también evidencias de un comportamiento sísmico negativo de estos muros, bien por provocar torsiones debidas a efectos de rigidización que desplazan el centro de torsión del centro de masas, bien por inducir concentraciones de daño en las plantas que no disponían de muros, bien por salir violentamente expulsados del plano del pórtico en los primeros ciclos de carga que impone el terremoto, por provocar efectos "columna corta", entre otras causas. Aunque existen estudios sobre cómo influyen los muros de cerramiento o particiones en la respuesta sísmica de estructuras porticadas, son escasas las investigaciones centradas en emplear muros de fábrica de ladrillo como solución para reacondicionar sísmicamente estructuras existentes, antes o después de haber experimentado un terremoto severo. Este trabajo se centra en la aplicación de los muros de fábrica de ladrillo para reacondicionar estructuras porticadas de hormigón armado (HA) de baja o media altura (hasta 2-3 plantas).

Investigaciones previas han puesto de manifiesto que el comportamiento bajo cargas cíclicas de los muros de fábrica de ladrillo enmarcados en pórticos de HA es menos favorable que el de otras soluciones más avanzadas como por ejemplo la adición de disipadores de energía. Los muros presentan unas curvas fuerza-desplazamiento lateral con un fuerte efecto de "pinzamiento" (pinching effect), y una capacidad de deformación plástica limitada, lo cual conduce a una capacidad límite última de disipación de energía mucho menor que, por ejemplo, los disipadores basados en la plastificación de elementos metálicos o los de tipo viscoso o viscoelástico. Sin embargo, entre las ventajas de emplear muros de fábrica de ladrillo para reacondicionar sísmicamente estructuras existentes de HA de poca altura están el considerable aumento de resistencia lateral que aportan, y su bajo coste económico. Esta segunda razón unida a su reducido nivel tecnológico las convierte en una solución muy adecuada para su empleo masivo, especialmente cuando los recursos económicos son limitados o en países en vías de desarrollo.

Un ejemplo claro y muy reciente de la necesidad de soluciones de reacondicionamiento sísmico económicas y de bajo nivel tecnológico es Haití, donde el terremoto de 2010 ha dejado miles de estructuras porticadas de HA con daños estructurales importantes (entre ellas muchos colegios) que requieren una reparación a muy corto plazo y con un coste reducido. Esta investigación puede contribuir también al conocimiento del comportamiento sísmico de la tabiquería en los edificios, tanto en España como en otros muchos países donde se emplean soluciones constructivas similares a las de nuestro país para resolver los cerramientos exteriores y las particiones interiores. Conviene resaltar que la participación de estos elementos no se tiene en cuenta normalmente a la hora de realizar el proyecto sísmico de una 
estructura, pero su influencia en la respuesta puede llegar a ser muy significativa (tanto en sentido positivo como negativo).

En este contexto, en este trabajo se investiga el empleo de muros de fábrica de ladrillo como solución de bajo coste para el reacondicionamiento sísmico de estructuras de hormigón armado de hasta tres plantas de altura. El trabajo se centra y aplica al caso concreto de los edificios de uso educativo que fueron seriamente dañados en Haití durante el reciente terremoto sufrido por este país. El objetivo es proponer disposiciones cualitativas (distribución en planta) y cuantitativa (área de muros en relación al área total en planta del edificio) adecuadas para garantizar un determinado nivel de comportamiento de la estructura bajo la acción del terremoto de proyecto.

\section{Metodología}

La metodología a utilizar está basada en el uso de cálculos dinámicos directos, que nos permiten hacer una evaluación paso a paso de la respuesta de la estructura durante el terremoto de proyecto, y cuantificar parámetros importantes de la respuesta como el desplazamiento máximo entre plantas, índices de daño etc.

Se trata de evaluar una selección de edificios representativos de Haití destinados a escuelas, para ello se aborda su concepción original, las consecuencias que ha podido tener el terremoto de Febrero de 2010 en el comportamiento frente al sismo de sus elementos estructurales y la mejora de su comportamiento frente al sismo añadiendo muros de ladrillo a su estructura, inicialmente formada por pórticos desnudos. Se pretende que las estructuras reacondicionadas sísmicamente con muros de fábrica de ladrillo respondan adecuadamente para el nivel de peligrosidad sísmica del país. Para llegar a este objetivo se procede en diversos apartados que serán descritos a lo largo de esta memoria.

En primer lugar se hace una clasificación de prototipos. Para ello se ha utilizado como base la documentación de campo obtenida por un equipo de investigación de la Universidad de Purdue [REF1], donde encontramos información sobre edificios en Haití, clasificados por usos, y en los que se distinguen los elementos estructurales que los conforman y sus dimensiones. Centrándonos en los edificios destinados a escuelas, se ha hecho una clasificación de aquellos cuyas dimensiones y distribución en planta son representativos del resto del conjunto. Puesto que estos edificios tienen de manera indiferente 2 o 3 alturas, se plantea estudiar cada uno de los prototipos para ambos casos. El proceso se detalla en el capítulo 3.

Una vez definida la geometría de los prototipos, luces de vigas, de crujías, alturas de plantas y dimensiones de las secciones de vigas y columnas se ha procedido al dimensionado de las secciones de hormigón armado con el programa Tricalc. Dada la ausencia de normativa para la construcción en Haití, se ha establecido una combinación de cargas que incluye solo cargas gravitatorias para una situación persistente según el CTE. Pensamos que esta combinación de cargas es la que se ha empleado en Haití para proyectar las escuelas que sufrieron daños en el último terremoto. El proceso se detalla en el capítulo 4. 
Una vez definida completamente la estructura (geometrías y armado) se procede a codificarla (modelizarla) para que pueda ser analizada con el programa de cálculo dinámico IDARC, con el cual se realizan los cálculos dinámicos directos.

IDARC permite dos modos de entrada de datos de secciones, bien a partir de la geometría y armado, o bien introduciendo directamente los diagramas momento-curvatura de las secciones y el diagrama de interacción momento-axil.

En este trabajo se ha empleado la segunda opción. Para ello, se ha analizado cada una de las secciones para obtener los gráficos momento-curvatura con el programa RESPONSE2000, desarrollado por la Universidad de Toronto, que permite obtener la capacidad resistente de secciones de pilares y vigas sometidas a momento, axil o cortante. En nuestro caso partiremos de que en el programa IDARC no se consideran los esfuerzos axiles en las vigas y por tanto la obtención del diagrama momento curvatura para una sección es suficiente. Por su parte, en los pilares sí se tiene en cuenta el axil que reciben, por lo que los diagramas a obtener son tanto el diagrama momento-curvatura como el diagrama de interacción axil momento para conocer la capacidad última de la sección. La hipótesis de partida de este programa es que las secciones planas permanecen planas. Se detalla el proceso en el capítulo 5 .

En cuanto a los muros de ladrillo el modelo numérico que utiliza el programa IDARC para el cálculo de propiedades mecánicas es el de Saneinejad y Hobbs (1995), desarrollado partiendo de que los muros de ladrillo están confinados por pórticos de acero. En el caso de las estructuras objeto de este estudio, los muros de ladrillo van a estar confinados por pórticos de hormigón armado. Es por esta razón que en este trabajo se ha preferido modelizar las propiedades mecánicas y el comportamiento cíclico de los muros utilizado valores experimentales obtenidos de los ensayos que tuvieron lugar en la Universidad de Purdue (2008) [REF.1].

Una vez modelizados los prototipos de estructuras a investigar, se ha procedido a la elección de acelerogramas y a definir criterios de escalado. El criterio de escalado elegido ha consistido en multiplicar el acelerograma por un factor de escala que haga que la aceleración máxima del suelo (Peak Ground Acceleration, PGA) sea igual a un valor predeterminado que se ha estimado a partir de la información sobre peligrosidad sísmica mundial publicada por el GSHAP y teniendo en cuenta el tipo de suelo e importancia de la estructura según criterios de la NCSE-02. Se detalla este proceso en el capítulo 6.

Los pórticos de hormigón armado (que representan a las estructuras existentes) con diferentes configuraciones de muros de fábrica de ladrillo se han analizado con la versión IDARC 2D V6.1 del programa IDARC. Este proceso se muestra a partir del capítulo 7.

\section{Cálculos dinámicos directos}

El estudio de la respuesta de cada edificio ante el sismo se evalúa mediante la realización de análisis dinámicos directos no lineales. El sistema de ecuaciones diferenciales que gobierna la respuesta de la estructura representa la presencia de los muros de ladrillo por el método de la pseudo-fuerza, y se resuelve mediante iteraciones por el método de Newmark. El sistema de ecuaciones de equilibrio en forma incremental se puede escribir como sigue: 
$[M]\{\Delta \ddot{u}\}+[C]\{\Delta \dot{u}\}+\left[K_{t}\right]\{\Delta u\}=-[M]\left(\left\{L_{h}\right\} \Delta \ddot{x}_{g h}+\left\{L_{v}\right\} \Delta \ddot{x}_{g v}\right)-\left\{\Delta P_{I W}\right\}+c_{c o r r}\left\{\Delta F_{e r r}\right\}$

Donde:

[M] es la matriz de masas generalizada de la estructura

[C] es la matriz de amortiguamiento de la estructura

$\left[K_{t}\right]$ es la matriz de rigidez secante

$\{\Delta u\},\{\Delta \dot{u}\}$ y $\{\Delta \ddot{u}\}$ Son los vectores de incremento de desplazamiento, velocidad y aceleración $\left\{L_{h}\right\}$ y $\left\{L_{v}\right\}$ son los vectores asignados a las aceleraciones del suelo horizontal y vertical respectivamente

$\Delta \ddot{x}_{g h}, \Delta \ddot{x}_{g v}$ son los incrementos de aceleración vertical y horizontal

$\left\{\Delta P_{I W}\right\}$ Son las fuerzas restauradoras correspondientes a los muros de ladrillo

$c_{c o r r}$ Es un coeficiente de correlación (normalmente igual a 1)

$\left\{\Delta F_{e r r}\right\}$ Es el vector de fuerzas que hay que añadir para garantizar el equilibrio en la estructura

Para obtener la solución de este sistema IDARC utilizan el algoritmo de Newmark-Beta

$$
\begin{aligned}
& \{\dot{u}\}_{t+\Delta t}=\{\dot{u}\}_{t}+\Delta t\left[(1-\gamma)\{\ddot{u}\}_{t}+\gamma\{\ddot{u}\}_{t+\Delta t}\right] \\
& \{u\}_{t+\Delta t}=\{u\}_{t}+\Delta t\{\ddot{u}\}_{t}+(\Delta t)^{2}\left[(0.5-\beta)\{\ddot{u}\}_{t}+\beta\{\ddot{u}\}_{t+\Delta t}\right.
\end{aligned}
$$

Donde $\beta$ y $\gamma$ son los parámetros del método de Newmark-Beta. Para eliminar el amortiguamiento artificial en los cálculos hay que tomar $\gamma=1 / 2$. En función del valor que se tome para $\beta$ el método de Newmark-Beta da lugar a dos variantes. Una variante consiste en hacer $\beta=1 / 4$ y con ello se asume que la aceleración dentro del intervalo de tiempo de un paso al siguiente es constante (método de la aceleración media constante). Esta versión del algoritmo es incondicionalmente estable. La otra variante consiste en hacer $\beta=1 / 6$ y con ello se asume que la variación de la aceleración dentro del intervalo es lineal (método de la aceleración lineal). La segunda versión del algoritmo es más eficiente que la primera pero es condicionalmente estable. Para evitar problemas de estabilidad con el método de la aceleración lineal el incremento de tiempo de un paso a otro debe ser inferior a Tn/1.8 siendo Tn el periodo del modo de vibración más alto del sistema.

Reordenando las ecuaciones anteriores obtenemos:

$$
\begin{aligned}
& \{\dot{u}\}_{t+\Delta t}=\left(1-\frac{\gamma}{2 \beta}\right) \Delta t\{\dot{u}\}_{t}-\frac{\gamma}{\beta}\{\dot{u}\}_{t}+\frac{\gamma}{\beta \Delta t}\{\Delta u\}_{t+\Delta t} \\
& \{\Delta \ddot{u}\}_{t+\Delta t}=\frac{1}{\gamma \Delta t}\{\dot{u}\}_{t+\Delta t}-\frac{1}{\gamma}\{\ddot{u}\}_{t}
\end{aligned}
$$

Sustituyendo estas expresiones en las ecuaciones de equilibrio se obtiene:

$$
\left[K_{D}\right]\{\Delta u\}_{t+\Delta t}=\left\{\Delta F_{D}\right\}
$$


Donde $\left[K_{D}\right]$ y $\left\{\Delta F_{D}\right\}$ son la rigidez dinámica equivalente y el vector de cargas

$\left[K_{D}\right]=\frac{1}{\beta(\Delta t)^{2}}[M]+\frac{\gamma}{\beta \Delta t}[C]+\left[K_{t}\right]$

$\left\{\Delta F_{D}\right\}=-[M]\left(\left\{L_{h}\right\} \Delta \ddot{x}_{g h}+\left\{L_{v}\right\} \Delta \ddot{x}_{g v}\right)-\left\{\Delta P_{I W}\right\}+c_{c o r r}\left\{\Delta F_{e r r}\right\}+\left(\frac{1}{2 \beta}[M]+\left(\frac{\gamma}{2 \beta}-\right.\right.$

1) $\Delta t[C])\{\ddot{u}\}_{t}+\left(\frac{1}{\beta \Delta t}[M]+\frac{\gamma}{\beta}[C]\right)\{\dot{u}\}_{t}$

El incremento de desplazamientos se obtiene al resolver el sistema 1.5. La velocidad y las aceleraciones se pueden calcular sustituyendo a partir de aquí en las ecuaciones 1.3 y 1.4 .

La resolución del sistema de ecuaciones se lleva a cabo en pasos incrementales, considerando que las propiedades de la estructura no cambian dentro de cada paso del análisis. En el momento en que la rigidez de algún elemento cambia, la nueva configuración no satisface el equilibrio. Para solventar esta diferencia y conseguir que el error sea mínimo, se lleva a cabo un procedimiento de compensación aplicando una fuerza de corrección en el paso en cuestión.

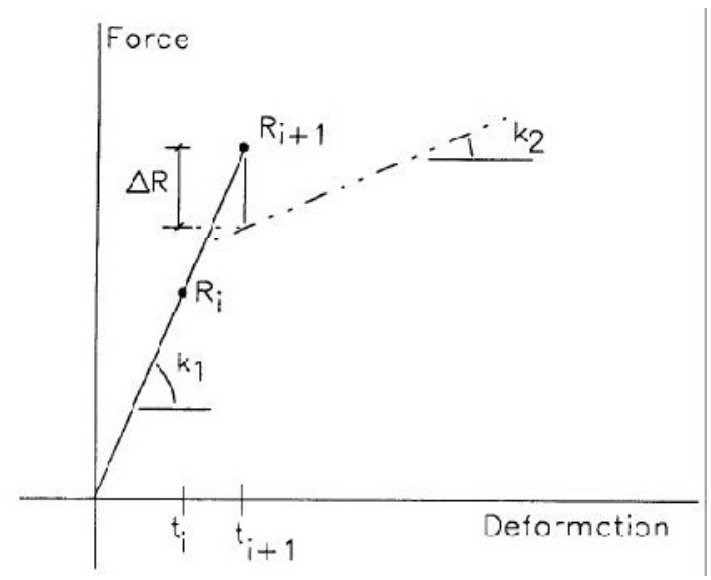

Figura 1

Así al final del paso $t+\Delta t$ la diferencia entre la fuerza restauradora calculada usando el modelo histerético $(\{R\})$, y la fuerza restauradora considerando que no hubiese cambios en la rigidez en ese paso concreto del análisis $\left(\left\{R^{\prime}\right\}\right)$ nos permite calcular la fuerza de desequilibrio:

$\left\{\Delta F_{\text {err }}\right\}=(\{R\})-\left(\left\{R^{\prime}\right\}\right)$

Esta fuerza correctiva se aplica al siguiente paso del análisis y permite la corrección para el cálculo de momentos, cortantes y rigideces en el modelo.

Este procedimiento permite que el cálculo dinámico no tenga un coste de tiempo prohibitivo...

Por su parte para el cálculo de la matriz de amortiguamiento de la estructura se considera que es una combinación lineal de la matriz de masas y de la matriz de rigidez (amortiguamiento de Rayleigh) para los dos primeros modos de vibración:

$$
[C]=\alpha_{M}[M]+\alpha_{K}[K]
$$

Siendo:

$$
\begin{aligned}
& \alpha_{M}=\frac{2 \varepsilon_{i} w_{i} w_{j}^{2}-2 \varepsilon_{j} w_{j} w_{i}^{2}}{w_{j}^{2}-w_{i}^{2}} \\
& \alpha_{K}=\frac{2 \varepsilon_{j} w_{j}-2 \varepsilon_{i} w_{i}}{w_{j}^{2}-w_{i}^{2}}
\end{aligned}
$$


Cuando el porcentaje de amortiguamiento es el mismo en ambos modos considerados $\left(\varepsilon_{i}=\varepsilon_{j}=\varepsilon\right)$, la expresión queda simplificada a:

$$
\begin{aligned}
& \alpha_{M}=\frac{2 \varepsilon w_{i} w_{j}}{w_{j}+w_{i}} \\
& \alpha_{K}=\frac{2 \varepsilon}{w_{j}+w_{i}}
\end{aligned}
$$

Este procedimiento se lleva a cabo elemento por elemento y en la estructura global.

El comportamiento de cada elemento queda representado por macromodelos para vigas y columnas que computan en la matriz de cada elemento. A continuación se ensamblan todas y cada una de las matrices de rigidez de cada elemento dando lugar a la matriz global de la estructura. El vector de cargas se calcula según el método de análisis escogido (cálculo dinámico directo) descrito anteriormente. Aunque se ha generado la matriz de rigidez global, en el análisis la matriz de rigidez de cada elemento es comparada con la obtenida tras aplicar el modelo histerético:

$$
\left\{\Delta F_{\text {err }}\right\}=(\{R\})-\left(\left\{R^{\prime}\right\}\right)
$$

En el caso en que haya habido cambios en al menos un elemento de la estructura, la matriz de rigidez global se vuelve a construir para reflejar estos cambios. Finalmente se aplica el vector de fuerzas obtenido anteriormente:

$$
\begin{aligned}
& \left\{\Delta F_{D}\right\}=-[M]\left(\left\{L_{h}\right\} \Delta \ddot{x}_{g h}+\left\{L_{v}\right\} \Delta \ddot{x}_{g v}\right)-\left\{\Delta P_{I W}\right\}+c_{c o r r}\left\{\Delta F_{e r r}\right\}+\left(\frac{1}{2 \beta}[M]+\right. \\
& \left.\left(\frac{\gamma}{2 \beta}-1\right) \Delta t[C]\right)\{\ddot{u}\}_{t}+\left(\frac{1}{\beta \Delta t}[M]+\frac{\gamma}{\beta}[C]\right)\{\dot{u}\}_{t}
\end{aligned}
$$

y finalmente se resuelve el sistema:

$$
\left[K_{D}\right]\{\Delta u\}_{t+\Delta t}=\left\{\Delta F_{D}\right\}
$$

para conocer el incremento de desplazamientos.

Finalizado un ciclo de carga o descarga se calcula el índice de daño de Park y Ang que permite evaluar el daño en cada elemento para cada ciclo como la suma de dos términos, el desplazamiento o giro del elemento con respecto al desplazamiento último y la energía histerética disipada con respecto a la energía histerética última que puede disipar.

Finalizado este paso se comienza el nuevo ciclo de carga o descarga. 


\section{ANTECEDENTES. BIBLIOGRAFÍA. ESTADO DEL ARTE}

Los muros de ladrillo forman parte de los edificios como elementos separadores entre las diferentes estancias de viviendas, oficinas, hospitales o escuelas. Formados por pequeños bloques de barro unidos entre sí por cemento, dan lugar a un panel que al quedar confinado por un pórtico de hormigón armado, puede variar su rigidez y resistencia lateral hasta aumentarlas en un $500 \%$ y un $100 \%$ respectivamente [REF. 1]. Sin embargo, este hecho no es considerado en las normativas sismorresistentes en el acondicionamiento sísmico de una estructura.

Estos elementos no son tenidos en cuenta en el cálculo por los proyectistas ni se les presta por ello demasiada atención durante su construcción, hecho que genera incertidumbre en el comportamiento frente al sismo de los edificios. Así se considera que el contenido de frecuencias de la respuesta está dominado por las de la estructura de hormigón armado, sin embargo, esto no es del todo cierto en el sentido de que la mayoría de la energía es disipada por los muros de ladrillo y el daño en vigas y columnas se reduce con la presencia de muros de ladrillo [REF.2], más concretamente diferentes ensayos revelan una disminución del $50 \%$ del periodo con respecto al valor original [REF. 3], [REF. 4], [REF. 5], pese a ello, las formas de los primeros modos de vibración no varían significativamente [REF. 4]. Por otra parte, el no considerar la presencia de los muros de ladrillo tiene como consecuencia que su ejecución no sea en todos los casos la apropiada, concretamente, no suele prestarse suficiente atención a la necesidad de que el confinamiento del muro se realice con especial cuidado en los bordes del mismo.

La presencia de los muros de ladrillo interaccionando con el pórtico de hormigón armado predeterminan el mecanismo inicial y el de colapso frente a acciones sísmicas. Ante cargas pequeñas o bajas el muro de ladrillo permanece en contacto con el pórtico, sin embargo, ante cargas laterales elevadas el muro queda despegado en su borde, con lo que se pierde la unión con el pórtico, y dada su baja resistencia a tracción, su aportación al equilibrio se limita a esfuerzos de compresión. Es por ello que el muro de ladrillo es representado en la literatura como dos barras diagonales con rigidez y resistencia a compresión equivalente a la del muro de ladrillo [REF.2].

El efecto de la presencia de muros de ladrillo mencionado anteriormente (aumento de la resistencia lateral en un $100 \%$ y de la rigidez lateral un $500 \%$ ) se ilustra en la figura que muestra los resultados de unos ensayos realizados recientemente en la Universidad de Purdue [REF.1]: 


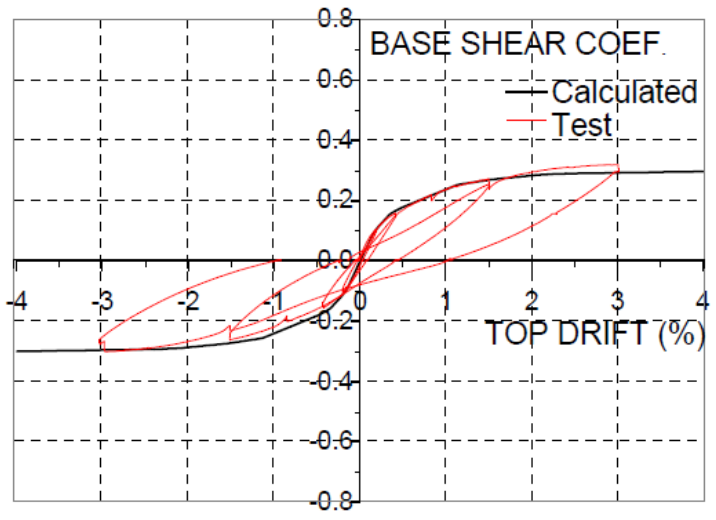

(a) Structure without walls

Figura 2 (a)

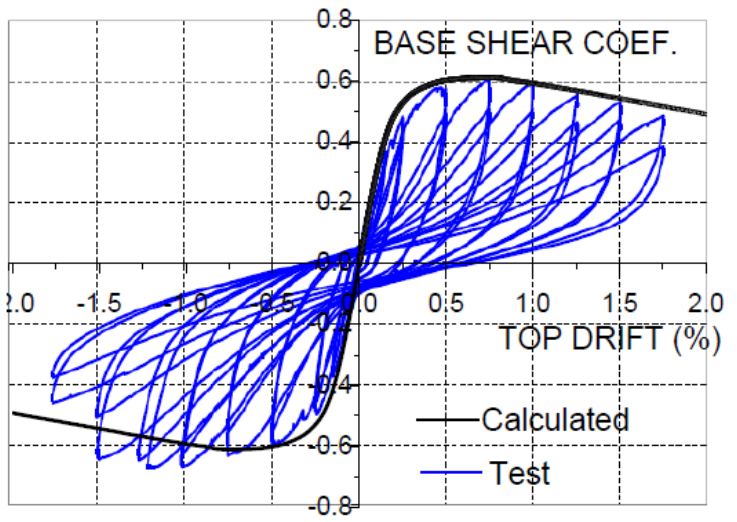

(b) Structure with walls

Figura 2 (b)

El la figura 2 (a) se muestran en rojo los ciclos histeréticos carga-deformación lateral obtenidos sometiendo una estructura de tres plantas a escala 1:1 formada por losas planas y pilares de hormigón armado, a cargas cíclicas. La curva en negro de la fig. 2 (a) es la predicción de la envolvente (curva de capacidad) obtenida numéricamente con el programa IDARC. La fig. 2 (b) muestra en azul los ciclos histeréticos carga-deformación lateral de la misma estructura anterior después de haberla reforzado con muros de fábrica de ladrillo.

Los resultados de ensayos recientes publicados en las referencias [REF. 1], [REF. 2], permiten relacionar el nivel de daño en el edificio con desplazamiento máximo entre plantas expresado como porcentaje de la altura de la planta. Esta relación se muestra en la Tabla 1

Tabla 1

\begin{tabular}{|c|c|}
\hline Desplazamiento máximo entreplantas & Nivel de daño \\
\hline $0.2-0.4 \%$ & Daños menores (puede seguir en servicio) \\
\hline $1.2-3.6 \%$ & Cercano al estado de colapso \\
\hline
\end{tabular}

También se puede relacionar el daño en el muro de ladrillo con el desplazamiento máximo entre plantas:

Tabla 2

\begin{tabular}{|l|l|}
\hline Desplazamiento máximo entre plantas & Daño en el muro \\
\hline $0.2 \%$ & Disminución de la rigidez \\
\hline $1 \%$ & Fisuras de $9.5 \mathrm{~mm}$ \\
\hline $1.75 \%$ & Fallo del muro \\
\hline
\end{tabular}

Además se han definido en la literatura, [REF.3], a partir de ensayos experimentales con un edificio de 6 plantas y 3 vanos, los estados de daño en el edificio que se resumen en la Tabla 3: 
Tabla 3

\begin{tabular}{|c|c|c|c|c|c|c|c|c|c|c|c|c|}
\hline \multirow{2}{*}{$\begin{array}{l}\text { Nümers } \\
\text { del especimen }\end{array}$} & \multicolumn{12}{|c|}{ Drift (comc porcentaje de la altura entre plantas) } \\
\hline & 1 & II & III & IV & $v$ & $\mathrm{VI}$ & VII & VIII & IX & $x$ & $\mathrm{XI}$ & XII \\
\hline 2 & ND & ND & ND & ND & ND & ND & ND & ND & 1,19 & 2,98 & ND & 3,7 \\
\hline 3 & 0,21 & 0,21 & 1,16 & 0,71 & ND & ND & ND & ND & ND & ND & ND & 3,2 \\
\hline 8 & $C, 2$ & 0,91 & 1,82 & 1,98 & ND & 1,49 & 1,59 & 1,98 & ND & ND & 2,48 & 3,1 \\
\hline 9 & 0,33 & 0,48 & 1,98 & 0,66 & ND & 82 & 2,07 & ND & ND & ND & 2,81 & 2,9 \\
\hline 12 & 0,17 & 0,55 & 1,02 & 0,95 & ND & 0,66 & 0,66 & ND & $\mathrm{ND}$ & ND & 1,65 & 1,7 \\
\hline
\end{tabular}

Donde:

I: grietas importantes en el muro de ladrillo o deslizamiento de la diagonal

II: Carga lateral máxima

III: Reducción del $80 \%$ de la carga lateral máxima

IV: grietas importantes de cortante en la columna

V: rotura a cortante en la unión viga-columna

$\mathrm{VI}$ : deslizamiento en las juntas de mortero

VII: aplastamiento en la parte interior del muro de ladrillo

VIII: aplastamiento en la esquina del muro de ladrillo

IX: plastificación de la armadura de refuerzo en la columna

X: plastificación de la armadura de refuerzo en la viga

$\mathrm{XI}$ : aplastamiento del hormigón de las columnas

XII: desplazamiento lateral máximo impuesto al espécimen

Se ha mantenido el nombre de los especímenes para mayor correlación con la fuente original. La abreviatura ND significa que no hay datos al respecto, porque no se ha alcanzado tal nivel de daño o porque no se ha podido medir.

Se han seleccionado de los doce pórticos analizados por el autor de la referencia [REF.3] aquellos que corresponden a pórticos desnudos, a los que tras ser sometidos a varios ciclos de carga y descarga, se les ha añadido muros de ladrillo para su estudio, por su relevancia con el trabajo que se desarrolla.

En términos energéticos, la presencia de muros de ladrillo permite que la cantidad de energía disipada por la estructura sea mucho mayor tal y como se muestra en los resultados que se muestran en las figuras 3 y 4 [REF. 4]. Esto ocurre pese al colapso progresivo o total de los muros de ladrillo en las dos primeras plantas: 


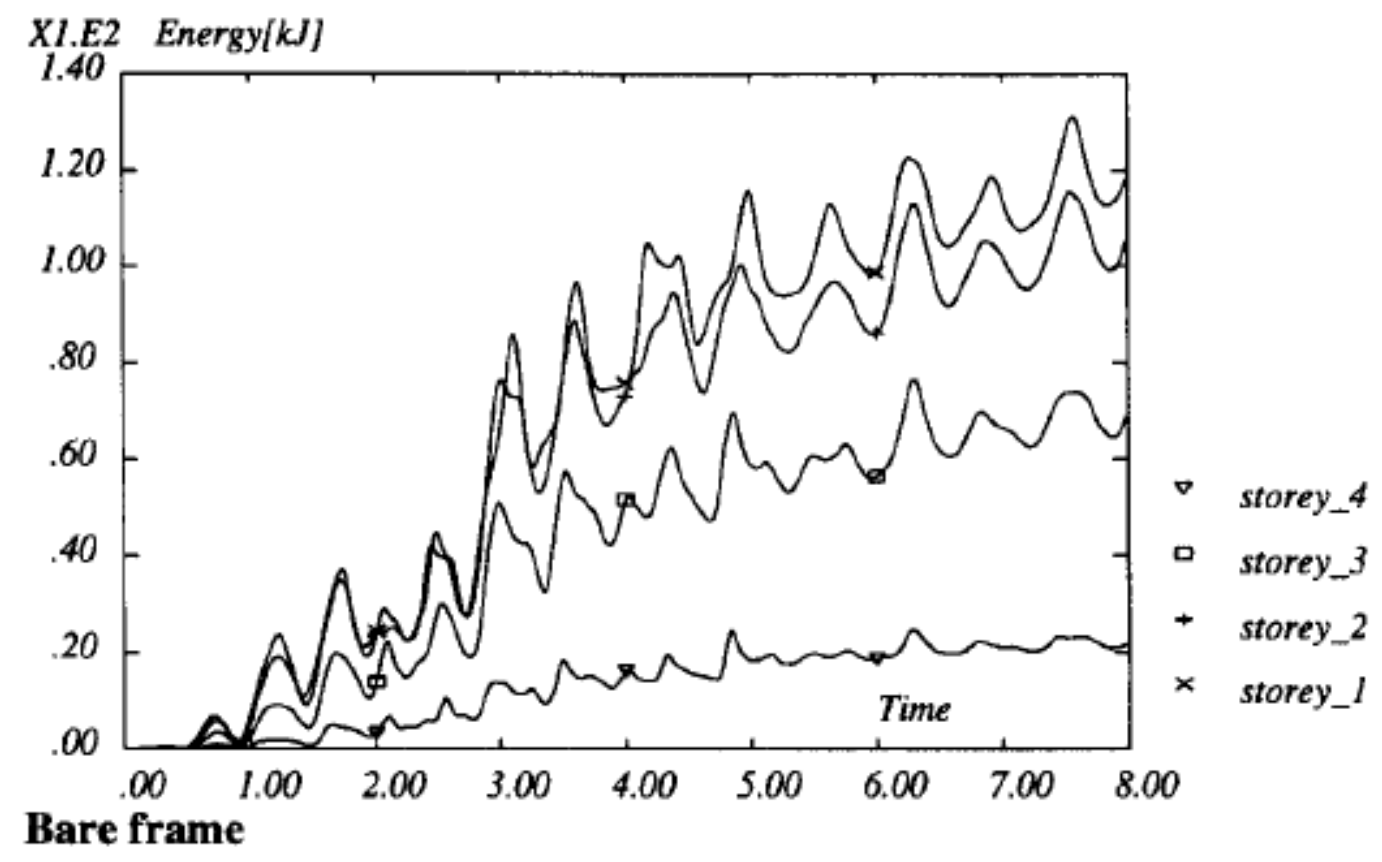

Figura 3

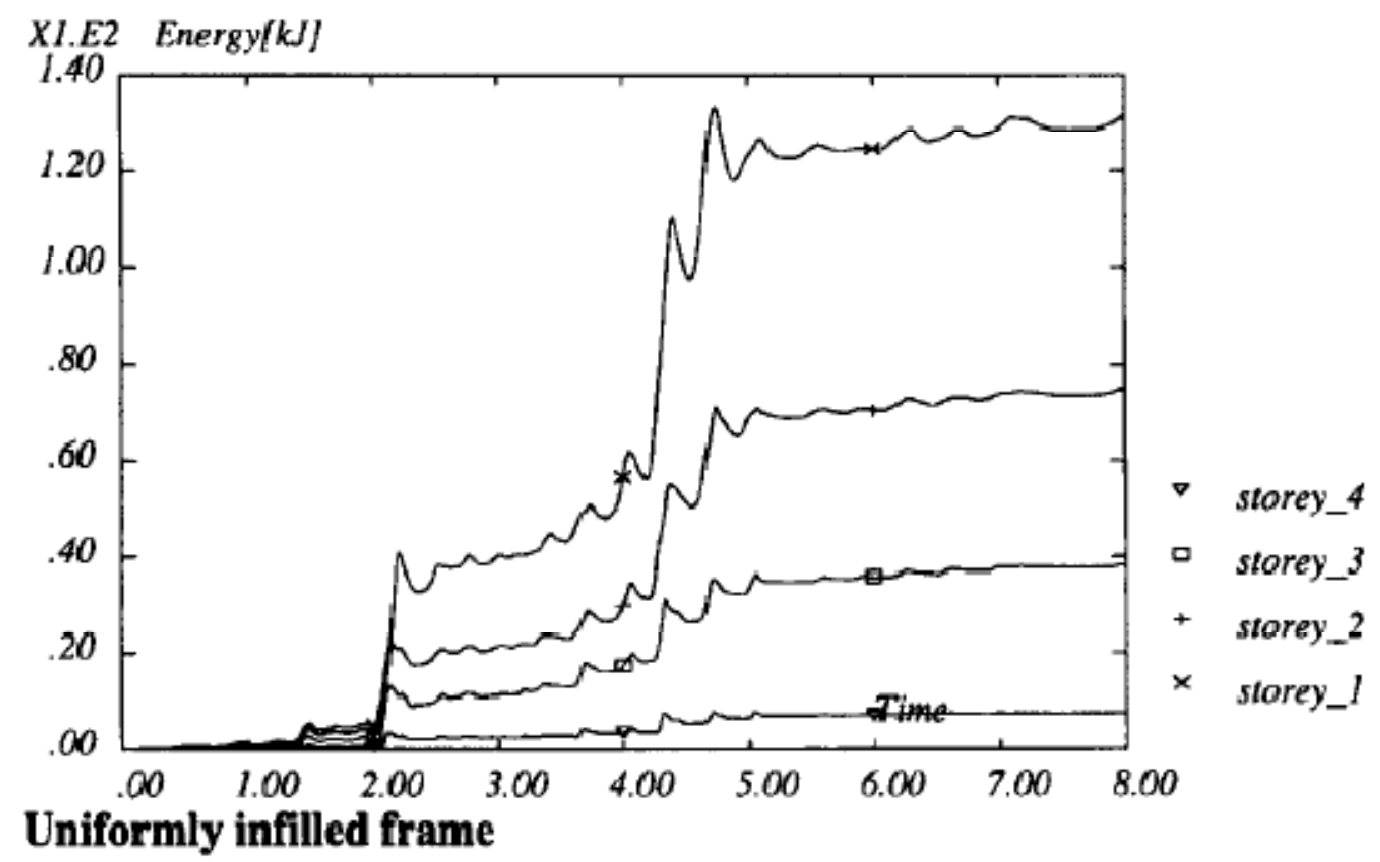

Figura 4

En las figuras 3 y 4 se muestra la energía total absorbida, como suma de la energía elástica y la energía histerética. Se observa en los gráficos anteriores que: (i) la energía capaz de disipar el pórtico aumenta cuando éste está reforzado con muros de ladrillo y (ii) se reduce la contribución a la disipación de energía de las plantas superiores, aunque ésta aún es importante.

Por último cabe hablar de la modelización de los muros de ladrillo confinados en un pórtico de hormigón armado. Como se ha mencionado anteriormente, los muros de ladrillo son 
idealizados normalmente como dos barras diagonales cuya contribución a la estructura es sólo ante esfuerzos de compresión.

El comportamiento histerético de las barras que representan a los muros de ladrillo confinados en un pórtico de hormigón armado se puede representar mediante el modelo de Wen-Bouc [REF. 6]. En este modelo la relación entre la fuerza lateral y el desplazamiento tiene dos componentes, las condiciones iniciales o elásticas y la componente histerética. Este modelo permite relacionar la fuerza y el desplazamiento. El comportamiento no lineal de los muros de ladrillo está dominado por tres fenómenos:

- Pérdida de rigidez

- Pérdida de resistencia

- Efecto pinzamiento

Estos efectos son introducidos en la formulación del modelo de Wen-Bouc con parámetros de control. La pérdida de rigidez es función de la ductilidad alcanzada.

La pérdida de resistencia está relacionada con la ductilidad y con la energía disipada en los ciclos anteriores de carga y descarga, todo ello resumido en el índice de daño propuesto por Reinhorn and Valles [REF. 7].

El efecto pinzamiento, debido a la apertura y cierre sucesivo de las grietas del muro, se idealiza con dos elementos en serie, el primero es un elemento con degradación suave y el segundo un muelle con rigidez 0 o infinita [REF.9]:

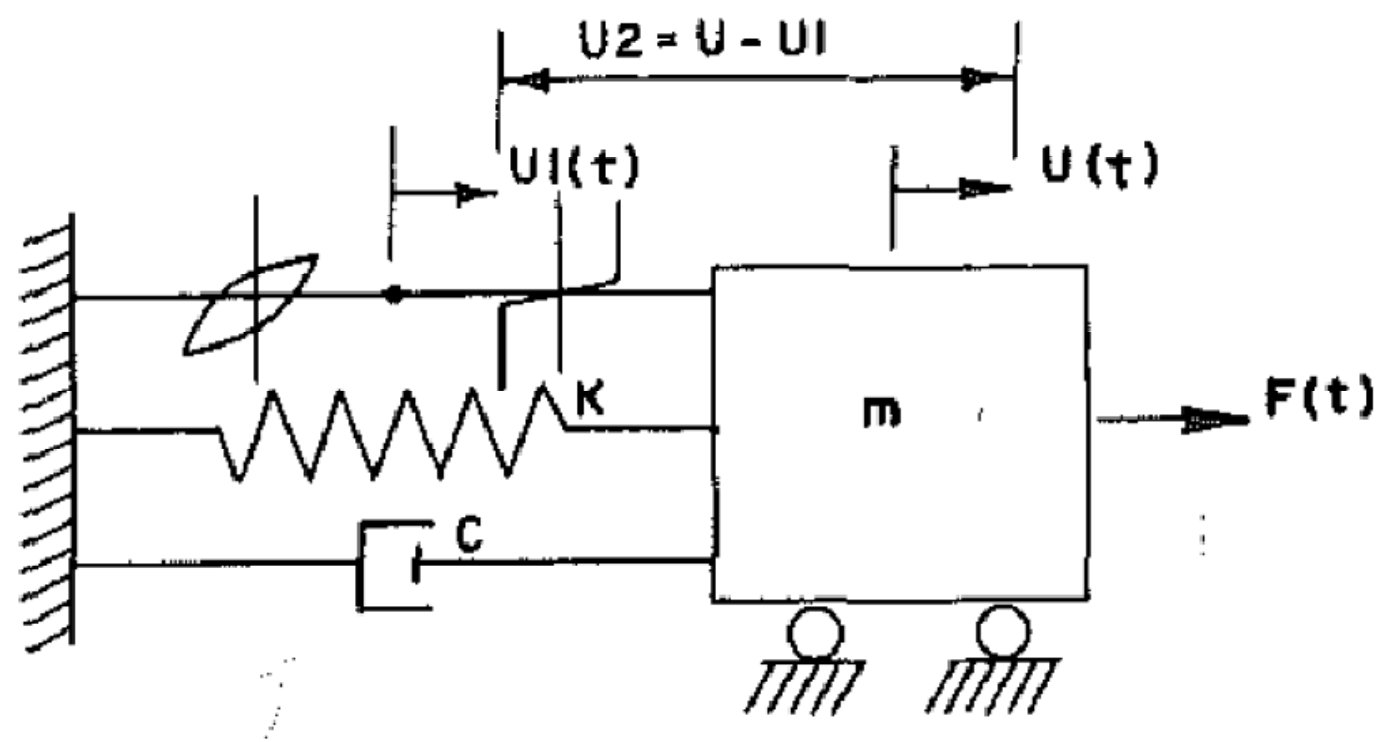

Figura 5

Esta modelización es ampliamente desarrollada en el capítulo 5. 


\section{DEFINICIÓN DE PROTOTIPOS DE ESCUELAS}

El estudio que se desarrolla pretende hacer una evaluación sobre la posibilidad de reacondicionamiento sísmico en edificios porticados de hormigón armado utilizando muros de ladrillo. El estudio se centra y aplica a edificios que resultaron dañados durante el reciente terremoto de Haití de febrero de 2010. La investigación parte de la información recopilada en un trabajo de campo en dicho país llevada a cabo pocas semanas después del terremoto, por un equipo de investigación de la Universidad de Purdue, liderado por el profesor Santiago Pujol [REF 1]. El profesor Santiago Pujol forma parte también desde 2005 del equipo de investigación de proyectos financiados por el Ministerio de Ciencia e Innovación BIA2005 00591, BIA2008 00050 y BIA2011 26816 que dirige el profesor Amadeo Benavent.

La información recopilada por el Prof. Santiago Pujol clasificada de alrededor de cien edificios inspeccionados, y se detalla para cada uno de ellos:

- Esquema en planta del edificio

- Número de plantas

- Tipo de estructura y dimensiones de los elementos estructurales

- Uso del edificio

A partir de esta clasificación, se han tomado en un primer lugar los edificios destinados a escuelas. El interés en este tipo de estructuras se debe a las siguientes razones: (i) corresponden a edificios cuyo reacondicionamiento sísmico es prioritario al ser estar destinados a escuelas; (ii) sus dimensiones y sus elementos constructivos son parecidos entre sí, y por lo tanto incluibles en una única tipología; (iii) son edificios de dos o tres plantas, para los cuales el reacondicionamiento sísmico con muros de fábrica de ladrillo parece, en principio, mucho más viable que para estructuras de más plantas. Por otra parte la rehabilitación sísmica en estos edificios tiene mucho sentido dado que están destinados a la educación que es la base de toda sociedad y en lo único en lo que pueden depositar sus esperanzas de desarrollo futuro. Encontramos un total de 21 edificios con estructura porticada de hormigón armado destinados a escuelas.

Para este tipo de edificios observamos además que los elementos estructurales son pórticos de vigas de canto $(30 \times 50 \mathrm{~cm})$ con forjado normalmente unidireccional, salvo el caso de luces de más de 7 metros, y pilares de sección cuadrada $(30 \times 30 \mathrm{~cm})$. La altura de planta de $3.05 \mathrm{~m}$.

Para desarrollar los prototipos de edificios representativos de escuelas en Haití, en primer lugar se analizó la distribución en planta, el número de vanos en cada dirección horizontal y luces de los edificios seleccionados. Esta información se resume en la Tabla 4: 
Tabla 4

\begin{tabular}{|c|c|c|c|c|c|c|c|c|}
\hline & Vanos X & Luz vanos $X \mathrm{~mm}$ & Luz vanos X mm (2) & Luz vanos X mm (3) & Vanos Y & Luz vanos $\mathrm{Y} \mathrm{mm}$ & Luz vanos Y mm (2) & NNe Plantas \\
\hline A001 & 1 & 7620 & & & 8 & 7620 & & 2 \\
\hline A002 & 8 & 4495,8 & 7239 & & 2 & 6096 & 4826 & 1 \\
\hline$A 003.1^{*}$ & 3 & 5029,2 & & & 4 & 3505,2 & 1524 & 3 \\
\hline$A 003.2^{*}$ & 4 & 3048 & & & 4 & 7188,2 & 914 & 2 \\
\hline A004 & 4 & 3911,6 & 2921 & & 5 & 3530 & 7112 & 2 \\
\hline B001 & 3 & 3302 & 1828 & 5359 & 10 & 7645 & & 2 \\
\hline B003 & 1 & 7543,8 & & & 5 & 7493 & & 4 \\
\hline B004 & 2 & 2794 & & & 5 & 3733 & 2997 & 2 \\
\hline B008 & $3 y 2$ & 2947 & 5207 & & 4 & 5359 & 5918 & 3 \\
\hline B009 & 4 & 1955 & & & 5 & 1711,96 & & 2 \\
\hline B010 & 3 & 3268 & & & 3 & 4318 & & 3 \\
\hline B011 & 5 & 2870 & 3429 & & 5 & 3810 & 4470 & $2 y 3$ \\
\hline B017 & 2 & 5003 & & & 4 & 3035 & & 2 \\
\hline B018 & 1 & 4242 & & & 4 & 3146 & & 2 \\
\hline Co01 & 1 & 7925 & & & 8 & 8128 & & 2 \\
\hline $\begin{array}{c}\mathrm{CO} \\
\end{array}$ & 4 & 6502 & & & 2 & 5359,4 & & 2 \\
\hline $\begin{array}{l}032 \\
\end{array}$ & 2 & 1752 & 4191 & & 5 & 3322 & & 1 \\
\hline 5 & 5 & 3658 & & & 1 & 4877 & & 3 \\
\hline 6 & 6 & 5029 & & & 2 & 3964 & 4420 & 2 \\
\hline 41 & 5 & 4419 & & & 2 & 4541 & & 3 \\
\hline
\end{tabular}

En la tabla 4, se han utilizado diferentes colores para diferenciar geometrías distintas, concretamente:

- El color amarillo muestra edificios con estructura extremadamente alargada en una de las direcciones, se trata de una disposición no recomendada por muchas normas sísmicas; de ahí la necesidad de reacondicionamiento sísmico y el interés en su estudio en esta investigación.

- El color verde sigue mostrando estructuras alargadas pero menos que las señaladas en amarillo. Las luces de los vanos son muy similares.

- El color rosa corresponde a la disposición geométrica más repetida y consta de dos vanos en una dirección y entre 4 y 6 vanos en la dirección perpendicular.

- El color azul muestra el tipo de disposición más cercana al cuadrado en planta

Los edificios objeto de estudio quedan englobados en estas cuatro categorías. A continuación, sintetizando información de luces y crujías, se han definido cuatro prototipos de organización de elementos estructurales en planta, que representan las categorías anteriores y que se resumen en la Tabla 5

Tabla 5 Tipologías de organización de elementos estructurales en planta

\begin{tabular}{|c|c|c|c|c|}
\hline Nombre & № Vanos X & Luz X(m) & № Vanos Y & Luz Y(m) \\
\hline H01 & 1 & 7 & 8 & 7 \\
\hline H02 & 1 & 4 & 5 & 5 \\
\hline H03 & 2 & 5 & 4 & 5 \\
\hline
\end{tabular}

Se observa en la Tabla 5 que no se han tenido en cuenta diferentes luces en una misma dirección por no ser frecuente en los edificios analizados, además cuando se da este caso, la diferencia es mínima. 
Puesto que el número de plantas de estos edificios varía entre dos y tres, cada uno de estos prototipos de la Tabla 5 ha sido analizado para ambos casos, generando finalmente ocho prototipos para el estudio numérico que se ilustran en las Fig. 6 a 9 y se resumen en la Tabla 6 .
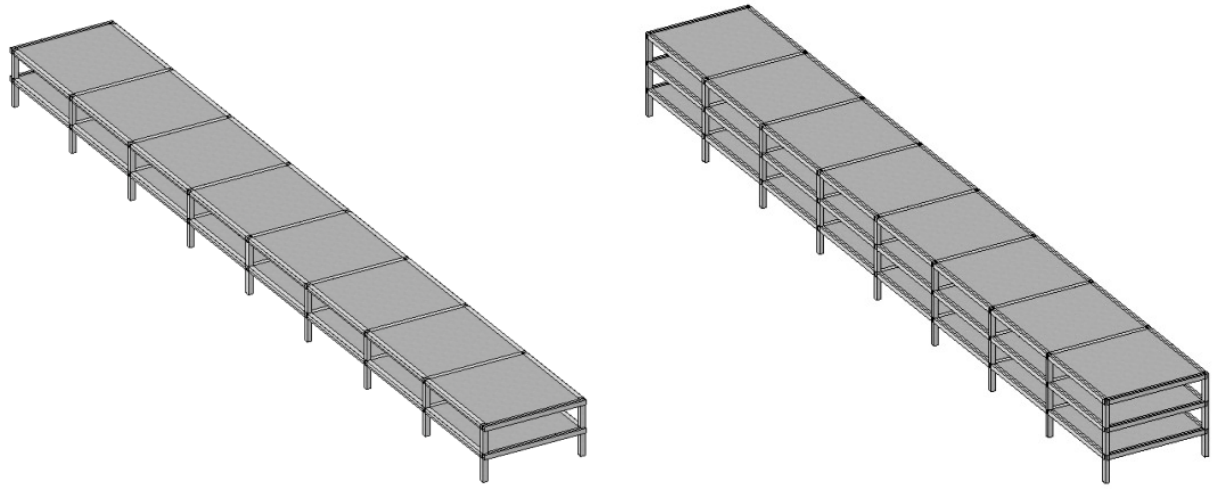

Figura 6. H01-1 y H01-2
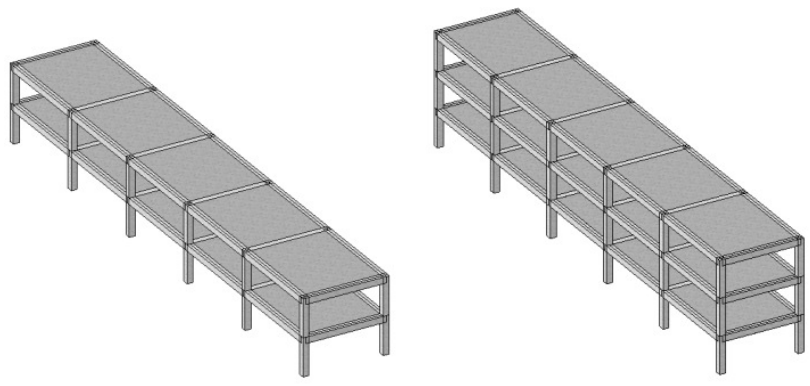

Figura 7. H02-1 y H02-2
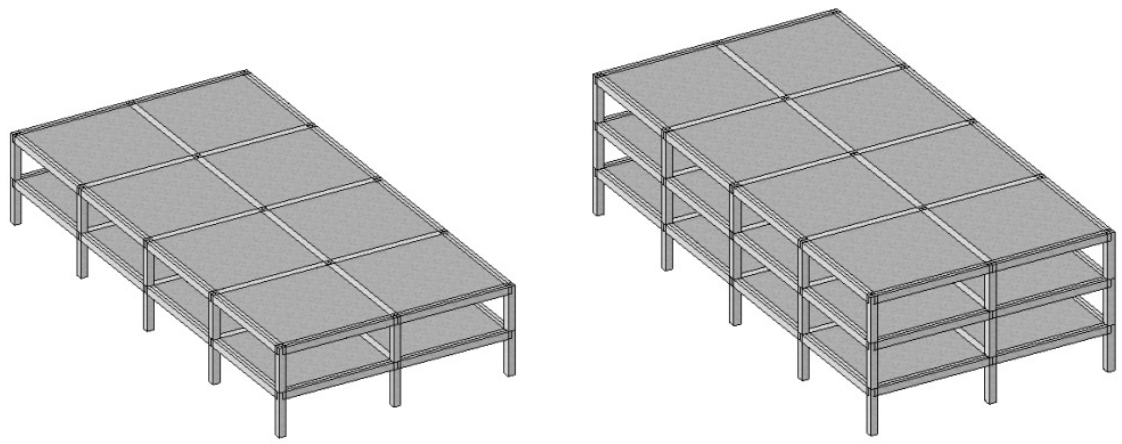

Figura 8. H03-1 y H03-2 

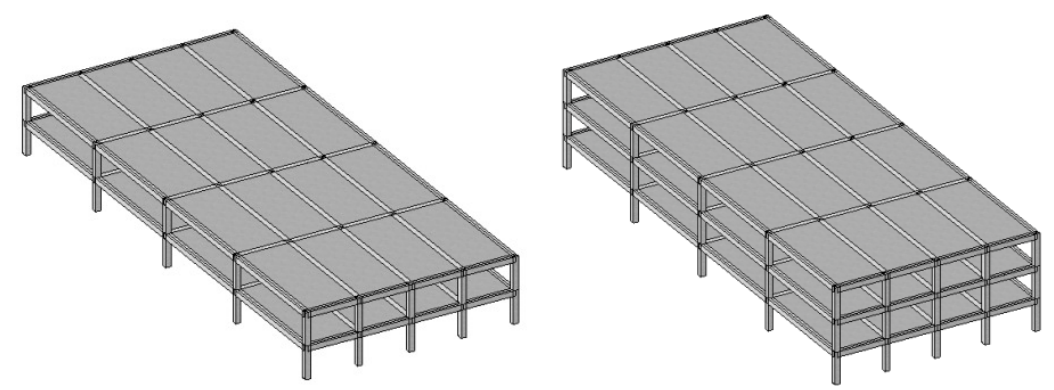

Figura 9. H04-1 y H04-2

Tabla 6. Prototipos de estructuras analizadas

\begin{tabular}{|c|c|c|c|c|c|}
\hline Nombre & № Vanos X & Luz X $(m)$ & № Vanos $Y$ & Luz Y $(m)$ & No. plantas \\
\hline H01-1 & & 7 & 8 & 7 & 2 \\
\hline H01-2 & 1 & 7 & 8 & 7 & 3 \\
\hline H02-1 & 1 & 4 & 5 & 5 & 2 \\
\hline H03-1 & 1 & 4 & 5 & 5 & 3 \\
\hline H03-2 & 2 & 5 & 4 & 5 & 2 \\
\hline H04-1 & 4 & 5 & 4 & 5 & 3 \\
\hline H04-2 & 4 & 3 & 4 & 7 & 2 \\
\hline
\end{tabular}

Se han nombrado las estructuras como sigue:

- Primer carácter inicial del proyecto en estudio: H - Haití

- El segundo carácter y primer número hace referencia al modelo de disposición en planta

- El tercer carácter, tras el guión, segundo número, hace referencia a la disposición en vertical:
○ 1: Dos alturas
○ 2: Tres alturas

- Se seguirá este orden para la denominación de los modelos tras introducir los muros de ladrillo, añadiendo a continuación una $\mathrm{M}$ y el número de muros que ha sido necesario introducir:

- MO: sin muros

- M1: relleno de muros en pórtico central

- M2: M1 más relleno de muros en pórticos extremos

- M3: M2con relleno de muros en pórticos centrales manteniendo la simetría. 


\section{DIMENSIONADO DE PROTOTIPOS EN TRICALC}

El predimensionamiento de la estructura para su posterior análisis frente a sismo se realiza con el programa Tricalc.

Tricalc permite el análisis de los pórticos de la estructura a partir del análisis de nudos y barras. Así, cada viga, pilar, o nervio de un forjado queda representado como una barra y las uniones entre éstas son los nudos, que se consideran infinitamente rígidos.

El programa realiza el cálculo de las solicitaciones por métodos matriciales, más concretamente por el método de la rigidez. El método consiste en la determinación, a partir de un sistema de ecuaciones lineales, de los desplazamientos y giros de todos los nudos y nodos de la estructura, frente a las distintas hipótesis de carga. Posteriormente se calculan los esfuerzos en todos los puntos de las barras y de los elementos finitos a partir de los desplazamientos y giros obtenidos en los nudos y en los nodos. Por último el programa comprueba el equilibrio local y global de la estructura.

La sección adoptada para las vigas es de $30 \mathrm{~cm} \times 50 \mathrm{~cm}$ de acuerdo a la información de campo consultada. La sección de los pilares es de $30 \mathrm{~cm} \times 30 \mathrm{~cm}$ en base a la misma fuente y las viguetas de los forjados unidireccionales están orientadas en la dirección del sismo en estudio.

Se ha establecido un coeficiente de empotramiento de 0.3 en los pilares ubicados en las esquinas de la planta superior, mientras que el resto de los nudos se les supone un coeficiente de empotramiento de 1 . El edificio está empotrado en su base, simulando la condición externa de la cimentación.

\section{Características de los materiales}

Se consideran los siguientes propiedades mecánicas para los materiales: Hormigón HA-25, $f_{c k}=25 \mathrm{MPa}, \gamma_{\mathrm{c}}=1.5$, nivel de control Normal

Acero B $400 \mathrm{~S}, \mathrm{f}_{\mathrm{yk}}=400 \mathrm{MPa}, \mathrm{\gamma}_{\mathrm{s}}=1.15$, Nivel de control Normal

\section{Cálculo de las acciones}

Para el cálculo de las acciones, en ausencia de normativa en Haití, se ha tomado la combinación de cargas gravitatorias del CTE para una situación persistente. No se han considerado cargas sísmicas porque los edificios en Haití se proyectaban sólo para cargas gravitatorias. Teniendo en cuenta que la única acción variable que se va a considerar es la sobrecarga la combinación de cargas queda como sigue:

$$
\sum \gamma_{G, j} \cdot G_{k, j}+\gamma_{Q, 1} \cdot Q_{k, 1}
$$

Siendo $\gamma_{G, j}=1.35$ (desfavorable); 0.8(favorable)

$$
\gamma_{Q, 1}=1.5 \text { (desfavorable); } 0(\text { favorable })
$$

Como ya se ha indicado anteriormente las cargas horizontales correspondientes al sismo y al viento no son consideradas en el proyecto de estas estructuras. 
El valor de las cargas es el siguiente:

$\mathrm{G}_{\mathrm{k}, \mathrm{j}}$ es el peso propio de los elementos estructurales (vigas, columnas y forjados) y las acciones permanentes.

Para la carga permanente se toma un valor de $2 \mathrm{KN} / \mathrm{m}^{2}$ correspondiente al falso techo y al solado.

$\mathrm{Q}_{k, 1}$ es la sobrecarga de uso para el caso de plantas distintas de la superior y la sobrecarga correspondiente a la cubierta para la planta superior.

Según el CTE, para las plantas intermedias se considera una sobrecarga de uso tipo C1, correspondiente a zonas con mesas y sillas, de valor $3 \mathrm{KN} / \mathrm{m}^{2}$. Para la planta superior se considera una cubierta accesible sólo para tareas de conservación y con inclinación inferior a 20 grados, lo que corresponde a una sobrecarga de $1 \mathrm{KN} / \mathrm{m}^{2}$.

Con esta información y la definición geométrica de las estructuras, mediante el programa Tricalc se obtuvo el armado de las barras, necesario para desarrollar los modelos numéricos que posteriormente se analizarán con el programa Idarc.

En el anexo a este trabajo llamado "Planos de estructura" se puede encontrar el dimensionado y armado completo de cada una de las estructuras prototipo investigadas. 


\section{DESARROLLO DE MODELOS NUMERICOS DE LOS PROTOTIPOS PARA SU ANÁLSIS DINÁMICO CON EL PROGRAMA IDARC}

El programa IDARC [REF. 18, sección 3.1] permite el estudio de la respuesta no lineal de estructuras de hormigón armado en 2D de varias plantas Como hipótesis de partida, se considera que los diafragmas horizontales son infinitamente rígidos en su plano, lo que implica que los desplazamientos horizontales de las vigas de una misma planta de todos los pórticos son iguales. Esta hipótesis nos permite ir más allá en el modelado, y puesto que los pórticos están unidos rígidamente, se pueden representar en un mismo plano vertical unidos entre sí por vigas biarticuladas e infinitamente rígidas, tal como se ilustra en la Fig. 10. No se consideran por tanto los posibles efectos de torsión.

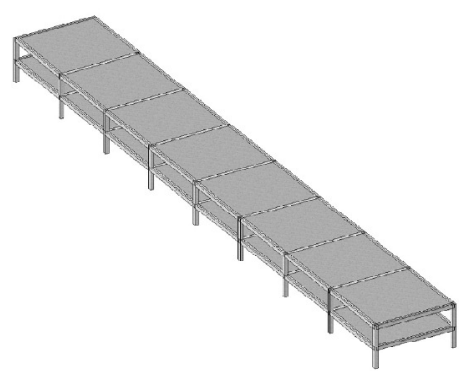

\begin{tabular}{|l|l|l|l|l|l|l|l|l|l|l|l|l|l|l|l|l|}
\hline & & & & & & & & & & & & & & & & \\
\hline & & & & & & & & & & & & & & & &
\end{tabular}

Figura 10. Idealización de la estructura 3D mediante una serie de pórticos planos paralelos unidos por barras ficticias biarticuladas de rigidez infinita (barras en color rosa)

El arranque de los pilares son empotramientos prefectos y existe simetría del edificio en la dirección del sismo. Se desprecia la contribución de los muros de ladrillo perpendiculares a la dirección del sismo.

En primer lugar se va a proceder a la descripción de los elementos estructurales (vigas y pilares) de los pórticos que integran los prototipos. Como ya se ha comentado en capítulos anteriores, para la realización de un cálculo dinámico en IDARC es necesario en primer lugar determinar la matriz de rigidez local de cada elemento. A continuación esta matriz se ensambla para obtener la matriz global de la estructura. Cuando un determinado paso k es llevado a cabo, se comprueba si la respuesta al sistema de fuerzas y desplazamientos para cada elemento es la misma calculada con la rigidez del paso anterior o con la rigidez que prescribe la ley histerética en ese momento. Si existe diferencia en estos valores se entiende que ha cambiado la matriz de rigidez del elemento y se reajusta a valores coherentes para el siguiente paso. Este reajuste es incorporado a la matriz de rigidez global. Finalmente se forma el sistema de ecuaciones que gobiernan el movimiento y los desplazamientos en cada paso.

Para la resolución de las ecuaciones del movimiento se aplica el método de pseudo-fuerzas y se resuelve integrando por el método de Runge-Kutta. Así los elementos estructurales como vigas y columnas computan como fuerzas internas en el sistema mientras que los muros de ladrillo computan como fuerzas externas. 


\section{Obtención de la matriz de rigidez para vigas y columnas}

La matriz de rigidez para elementos viga y columna se diferencia a efectos de cálculo solamente en el hecho de que se desprecia el esfuerzo axil en las vigas. .

Para desarrollar la matriz de rigidez en vigas y columnas se utiliza el mismo macromodelo, que se ilustra en la Fig. 11 y 12 :

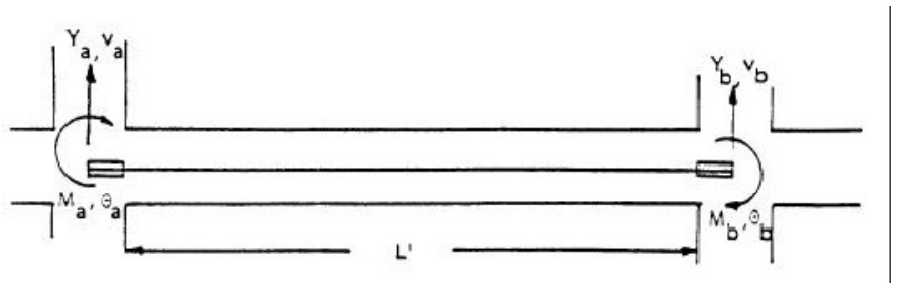

Figura 11

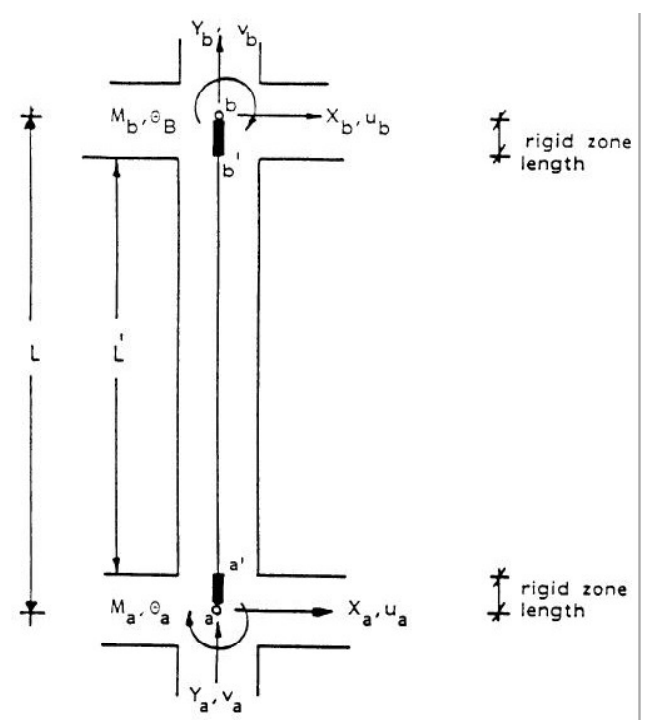

Figura 12

La relación entre el momento y la curvatura en cada extremo viene dada por:

$$
\left\{\frac{M_{a}^{\prime}}{M_{b}^{\prime}}\right\}=\left[K^{\prime}\right]\left\{\frac{\theta_{a}^{\prime}}{\theta_{b}^{\prime}}\right\}
$$

Donde $M_{a}^{\prime}$ y $M_{b}^{\prime}$ son los momentos en las caras a y $\mathrm{b}$ del elemento estructural, $\theta_{a}^{\prime}$ y $\theta_{b}^{\prime}$ son los giros en las caras a y $b, y\left[K^{\prime}\right]$ es la matriz de rigidez a esfuerzo flector y cortante básica del elemento, calculada según el modelo de "plasticidad dispersa" (spread plasticity model):

$$
\left[K^{\prime}\right]=\left[\begin{array}{ll}
k_{a a} & k_{a b} \\
k_{b a} & k_{b b}
\end{array}\right]
$$

donde:

$$
k_{a a}=\frac{12 E I_{0} E I_{a} E I_{b}}{D_{e t} L}\left(f_{b b}^{\prime} G A_{z} L^{2}+12 E I_{0} E I_{a} E I_{b}\right)
$$




$$
\begin{gathered}
k_{a b}=k_{b a}=-\frac{12 E I_{0} E I_{a} E I_{b}}{D_{e t} L}\left(f_{a b}^{\prime} G A_{z} L^{2}+12 E I_{0} E I_{a} E I_{b}\right) \\
k_{b b}=\frac{12 E I_{0} E I_{a} E I_{b}}{D_{e t} L}\left(f_{a a}^{\prime} G A_{z} L^{2}+12 E I_{0} E I_{a} E I_{b}\right)
\end{gathered}
$$

y $\mathrm{El}_{0}$ es la rigidez a rotación elástica, $\mathrm{El}_{\mathrm{a}}$ y $\mathrm{El}_{\mathrm{b}}$ la rigidez a rotación tangente en los extremos del elemento, $\mathrm{GA}_{z}$ es la rigidez a cortante, $\mathrm{L}$ la longitud referida al esquema anterior.

Además las vigas y columnas, incluyen una zona rígida que simula el incremento de rigidez en el nudo. Por relaciones geométricas se puede obtener el momento en los nodos rígidos mediante la siguiente transformación:

$$
\begin{aligned}
& \left\{\begin{array}{l}
M_{a} \\
M_{b}
\end{array}\right\}=[\tilde{L}]\left\{\begin{array}{l}
M_{a}^{\prime} \\
M_{b}^{\prime}
\end{array}\right\} \\
& \left\{\begin{array}{l}
\theta_{a} \\
\theta_{b}
\end{array}\right\}=[\tilde{L}]^{\prime}\left\{\begin{array}{l}
\theta_{a}^{\prime} \\
\theta_{b}^{\prime}
\end{array}\right\}
\end{aligned}
$$

Siendo:

$$
\left[L^{\prime}\right]=\frac{1}{1-\lambda_{a}-\lambda_{b}}\left[\begin{array}{cc}
1-\lambda_{b} & \lambda_{a} \\
\lambda_{b} & 1-\lambda_{a}
\end{array}\right]
$$

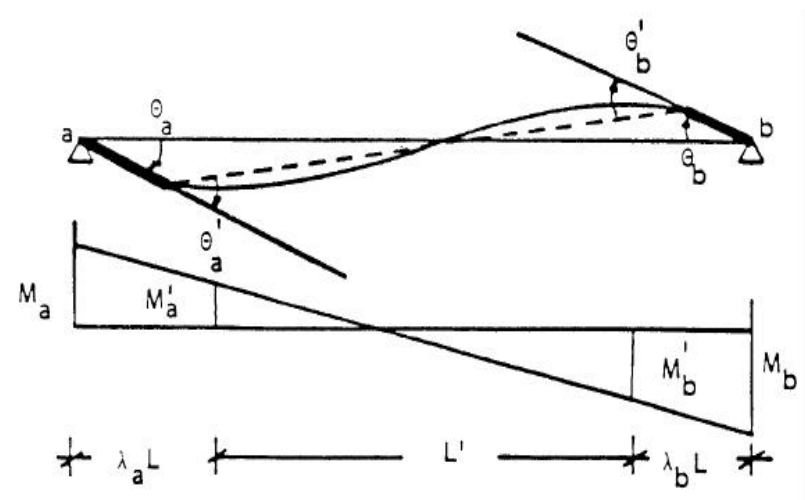

Figura 13

Donde $\lambda_{\mathrm{a}}$ y $\lambda_{\mathrm{b}}$ son la proporción de zona rígida con respecto a la longitud total del elemento en cada una de las caras $a \mathrm{y} b$.

Combinando estas ecuaciones, la ecuación básica que relaciona momentos y giros en los nodos del elemento viene dada por:

$$
\left\{\begin{array}{l}
M_{a} \\
M_{b}
\end{array}\right\}=\left[K_{s}\right]\left\{\begin{array}{l}
\theta_{a} \\
\theta_{b}
\end{array}\right\}
$$

Siendo

$$
\left[K_{S}\right]=[\tilde{L}]\left[K^{\prime}\right][\tilde{L}]^{t}
$$

\section{Comportamiento histerético de los elementos}

Por comportamiento histerético se entiende la relación entre la fuerza o momento aplicado y el efecto que produce (momento o giro) en un elemento estructural bajo ciclos de carga y descarga. El comportamiento histerético en elementos de hormigón armado está gobernado por tres efectos: degradación de rigidez, degradación de resistencia y pinzamiento. Estos tres efectos están controlados por los parámetros HC, HBR y HS que se ilustran en la Fig. 14. 


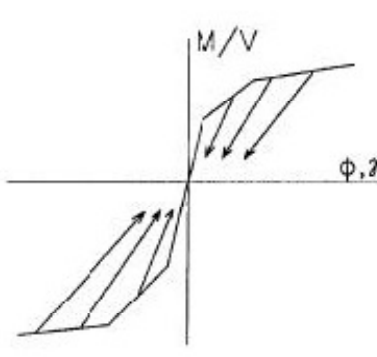

$H C=0.5$

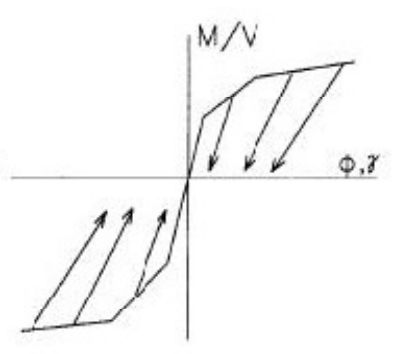

$H C=2.0$

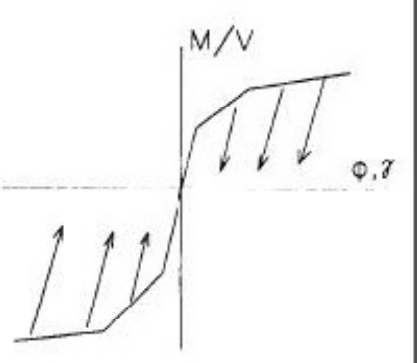

$H C=15.0$

Stiffness Degrading Parame:er

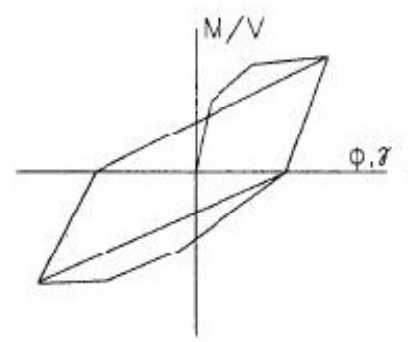

$\mathrm{HBE}=\mathrm{HBO}=0.0$

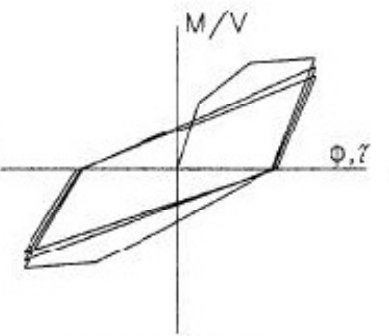

$\mathrm{HBE}=\mathrm{HBD}=0.12$

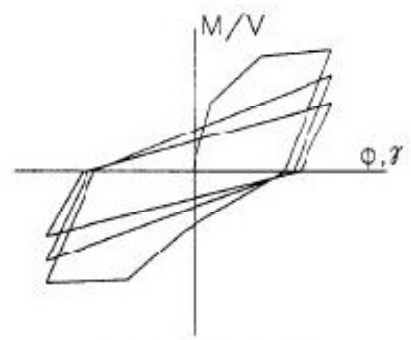

$H B E=H B D=0.40$

Strength Deterioration Parameter

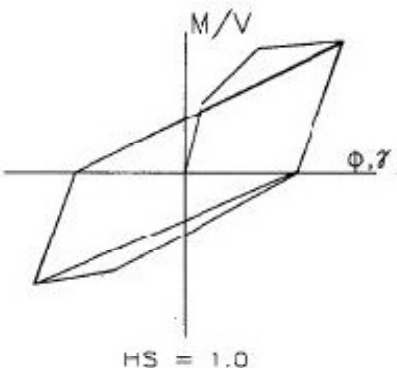

$H S=1.0$

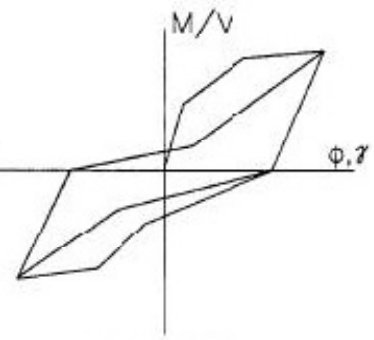

$H S=0.5$

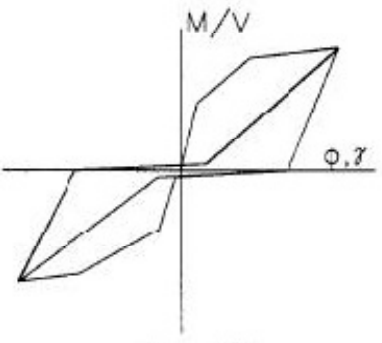

$H S=0.1$

Slip Control Porometer

Figura 14

El control de estos parámetros nos lleva a un gráfico que se muestra en la figura 15:

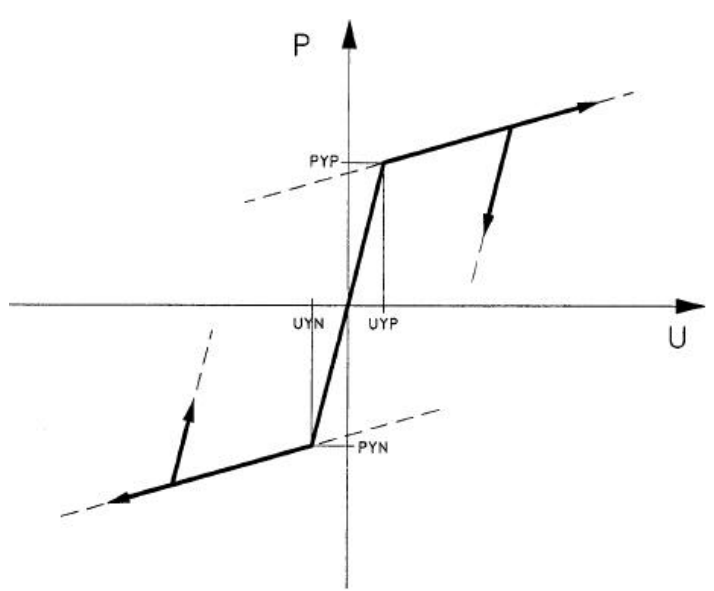

Figura 15 
Con respecto a los muros de fábrica de ladrillo, el comportamiento histerético se idealiza con el modelo "Smooth Hysteretic Model", el cual permite combinar el modelo de Bouc-Wen con parámetros que representen la degradación de la rigidez y la resistencia, así como el conocido efecto pinzamiento.

\section{Desarrollo del Smooth Hysteretic Model para los muros de mampostería}

Para el desarrollo teórico del modelo histerético del muro de mampostería, partimos del modelo de Wen-Bouc que será modificado para representar los efectos que tienen lugar en los muros de mampostería a causa de:

- Pérdida de rigidez

- Pérdida de resistencia

- Efecto pinzamiento

La Fig. 16 muestra la gráficamente el comportamiento del modelo "Smooth Hysteretic Model"

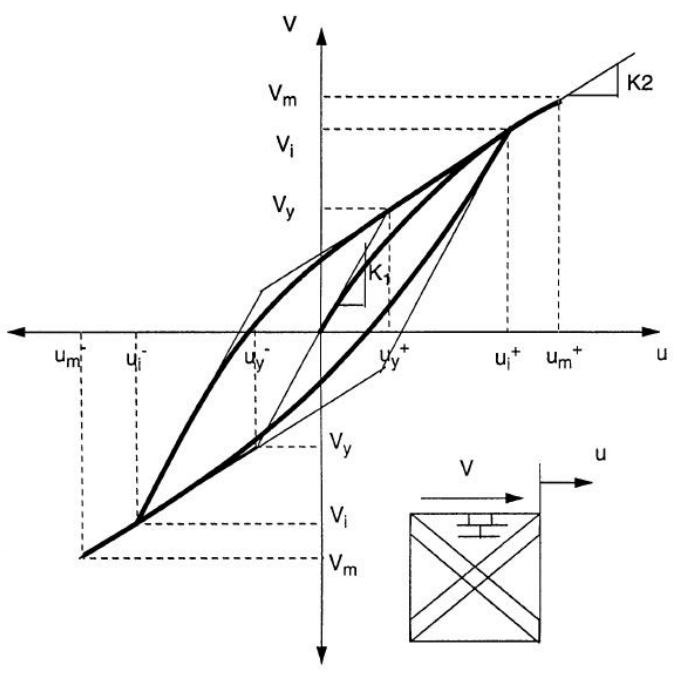

Figura 16

El modelo histerético que representa el comportamiento de cada muro de ladrillo tras los ciclos de carga y descarga parte de la ecuación que relaciona la fuerza y el desplazamiento y se define como sigue:

$V_{i}=V_{y}\left[\alpha \cdot \mu_{i}+(1-\alpha) Z_{i}\right]$

El subíndice i denotará a lo largo de todo el desarrollo valores instantáneos.

V esfuerzo

$V_{y}$ esfuerzo de rotura

$\alpha$ es el cociente entre la rigidez tras la rotura y la rigidez inicial

$\mu_{i}=\frac{u_{i}}{u_{y}}$ desplazamiento instantáneo normalizado con respecto al desplazamiento horizontal de rotura 
$\mathrm{Z}_{\mathrm{i}}$ es la componente histerética que viene dada por:

$$
\dot{Z}_{i}=\dot{\mu}_{i}\left[A-\left|Z_{i}\right|^{n}\left(\beta \cdot \operatorname{sgn}\left(\dot{\mu}_{i} \cdot Z_{i}\right)+\gamma\right)\right]
$$

Siendo: $\operatorname{sgn}\left(\dot{\mu}_{i} \cdot Z_{i}\right)=1$ si $\dot{\mu}_{i} \cdot Z_{i}>0$

Y $\operatorname{sgn}\left(\dot{\mu}_{i} \cdot Z_{i}\right)=-1$ si $\dot{\mu}_{i} \cdot Z_{i}<0$

Eliminando la derivada temporal y teniendo en cuenta que

$$
\operatorname{sgn}\left(\dot{\mu}_{i} \cdot Z_{i}\right)=\operatorname{sgn}\left(d \mu_{i} \cdot Z_{i}\right)
$$

Llegamos a la expresión:

$$
d Z_{i}=d \mu_{i}\left[A-\left|Z_{i}\right|^{n}\left(\beta \cdot \operatorname{sgn}\left(d \mu_{i} \cdot Z_{i}\right)+\gamma\right)\right]
$$

En esta expresión, $A, \beta$ y $\gamma$ son los denominados parámetros de control que permitirán ajustar la forma de la curva histerética, $n$ controla la velocidad de transición desde el estado elástico al de rotura, por ello con un alto valor de $\mathrm{n}$ obtendremos una curva bilineal mientras que valores bajos de $n$ nos permitirán una transición más suave.

\section{Pérdida de rigidez en los muros de mampostería:}

Una característica importante en los muros de ladrillo es la pérdida de rigidez que tiene lugar tras la rotura. El deterioro de la rigidez que ocurre tras los ciclos en los que el muro de ladrillo ha pasado al estado plástico puede expresarse en función de la ductilidad alcanzada, así se puede definir un parámetro que afecte directamente a la ecuación de partida:

$$
d Z_{i}=d \mu_{i} \frac{\left[A-\left|Z_{i}\right|^{n}\left(\beta \cdot \operatorname{sgn}\left(d \mu_{i} \cdot Z_{i}\right)+\gamma\right)\right]}{\eta_{i}}
$$

Siendo $\eta_{i}$ el parámetro de control de pérdida de rigidez que se define por:

$$
\eta_{i}=1+s_{k}\left(\frac{\mu_{\max }^{p}+\mu_{i}}{2}\right)
$$

Donde $s_{k}$ es un parámetro de control que determina la velocidad con la que se degrada la rigidez del muro en función de la ductilidad instantánea $\mu_{\mathrm{i}}$ y la máxima ductilidad alcanza antes del comienzo del ciclo de carga o descarga en que se reajusta la rigidez. Un valor de $s_{\mathrm{k}}$ igual a cero implicaría que no existe degradación de rigidez.

Pérdida de resistencia en los muros de mampostería:

Los muros de mampostería sufren también pérdida de resistencia al ser sometidos a ciclos de carga en rango inelástico. Esta pérdida de resistencia se puede introducir en el modelo variando la carga de rotura según el ciclo en que nos encontremos:

$$
V_{y}^{k}=s_{\beta} V_{y}^{0}
$$


Siendo $V_{y}^{k}$ Carga de rotura reducida para el ciclo $\mathrm{k}$

$V_{y}^{0}$ la carga de rotura inicial

$\mathrm{s}_{\beta}$ indica la cantidad de deterioro de la carga de rotura en el ciclo $\mathrm{k}$ con respecto a la original $\mathrm{y}$ tiene que ver con el daño acumulado por el muro de ladrillo, es por ello que se puede definir en función de un índice de daño como sigue:

$$
\mathrm{S}_{\beta}=1-\mathrm{DI}
$$

Este índice de daño es el propuesto por Reinhorn and Valles y es función de la ductilidad alcanzada y la energía disipada en el ciclo en cuestión:

$$
D I=\frac{\mu_{\max }^{p}-1}{\mu_{c}-1} \cdot \frac{1}{\left(1-\frac{{ }^{s} p 1 \int d E_{h}}{4 E_{h y}}\right)^{S_{p 2}}}
$$

Donde $\mu_{\max }^{p}$ es la máxima ductilidad alcanzada en la respuesta

$\mu_{\mathrm{c}}$ es la capacidad dúctil del muro de ladrillo

$\mathrm{S}_{\mathrm{p} 1}$ y $\mathrm{S}_{\mathrm{p} 2}$ controlan la velocidad de deterioro de la resistencia

$\int d E_{h}$ representa la energía disipada en el ciclo anterior al ciclo de carga actual

$E_{h y}$ es la capacidad de energía monotónica (energía disipada antes de la rotura)

$E_{h y}=V_{i} \cdot u_{i}\left(\mu_{c}-1\right)$

Por tanto, el índice de daño DI puede expresarse también como:

$$
D I=\frac{\mu_{\max }^{p}-1}{\mu_{c}-1} \cdot \frac{1}{\left(1-0.25 s_{p 1} \int\left(\frac{V}{V_{y}}\right) \frac{d \mu}{\mu_{c}-1}\right)^{S p 2}}
$$

El índice anterior refleja el ablandamiento acumulado debido a los numerosos ciclos en rango inelástico sin inversión de la carga así como la degradación de la resistencia debida a ciclos repetidos con pequeñas o moderadas deformaciones inelásticas.

\section{Efecto pinzamiento en los muros de mampostería}

El efecto pinzamiento tiene como origen físico la apertura y cierre de las grietas de un elemento que se encuentra sometido a ciclos de carga y descarga.

En 1985 Baber and Noori propusieron un modelo de degradación general para incorporar el efecto pinzamiento en la respuesta de un sistema de un grado de libertad. El modelo consiste en posicionar en serie un elemento de degradación suave (smooth degrading element) desarrollado por Wen-Bouc y un elemento slip-lock dependiente del tiempo (equivalente a un muelle con endurecimiento y comportamiento no lineal): 


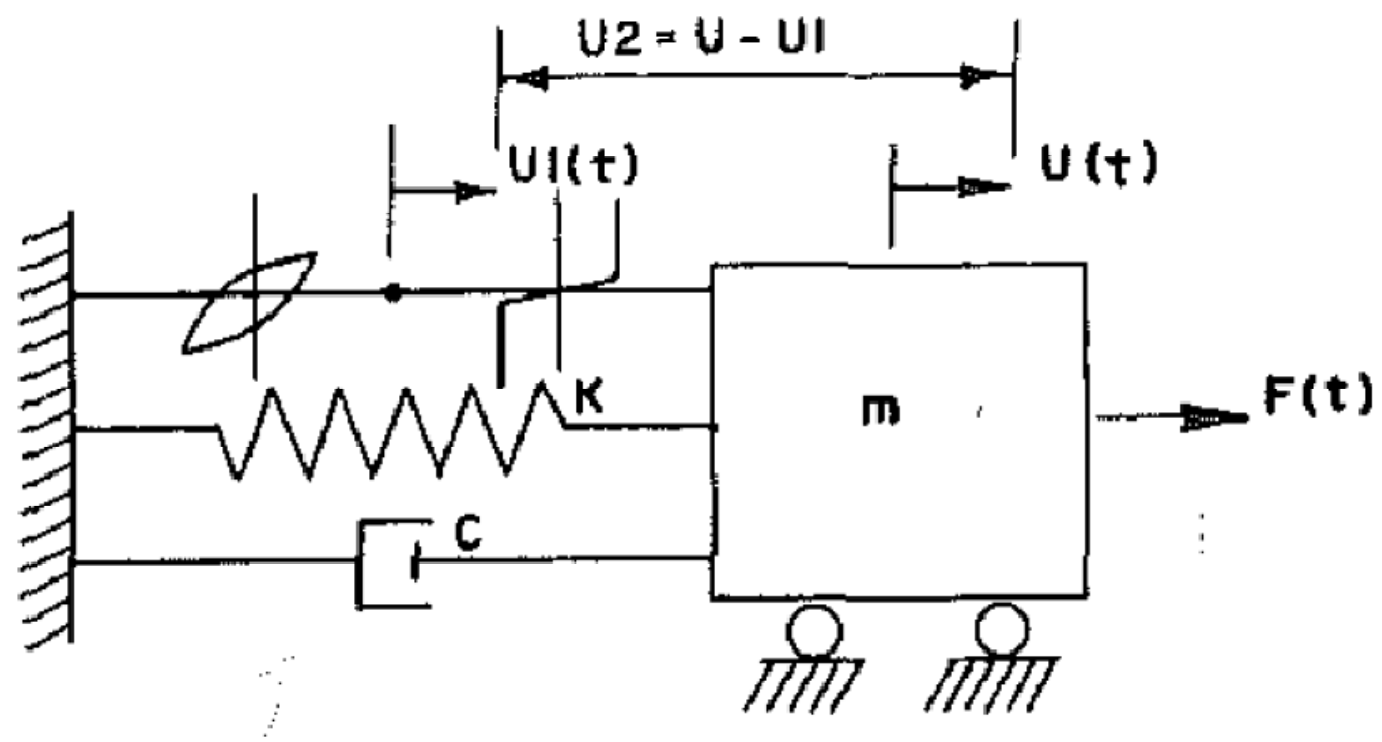

Figura 17 Modelo Baber \& Noori
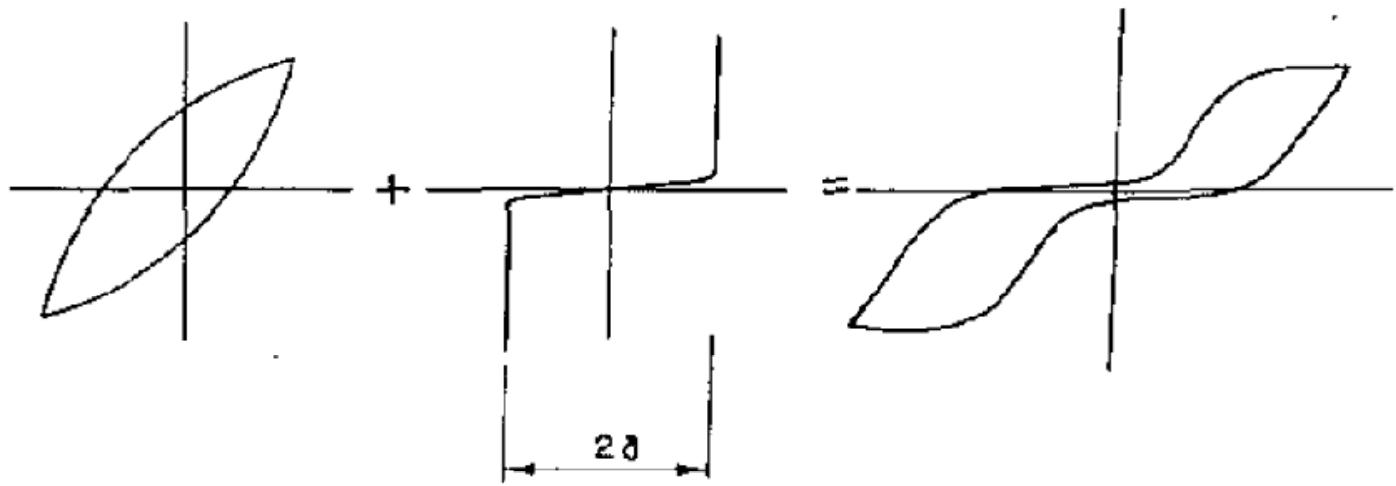

Figura 18

Este elemento se define por su rigidez que es prácticamente cero en la llamada zona "slip" (de longitud 2a) y tiende a infinito en la zona "locking".

La ecuación diferencial que relaciona la contribución del elemento slip-lock a la componente histerética $Z$ se escribe en términos de velocidad y se resuelve simultáneamente a las ecuaciones del movimiento para un sistema de un grado de libertad obteniendo la respuesta de un sistema degradado dinámicamente (en función del tiempo) por efecto pinzamiento.

La adaptación de la ley histerética al programa IDARC se establece partiendo de que la ley histerética que simula el comportamiento del elemento slip-lock es independiente de la historia de desplazamientos y puede escribirse para cualquier sistema de ecuaciones diferenciales. La formulación de la que se parte establece que el elemento slip-lock se encuentra ubicado en serie con el elemento degrading smooth, tal y como se muestra figura 18. El desplazamiento normalizado del elemento histerético pinzamiento smooth $\mu$ es la suma 
del desplazamiento normalizado para el elemento histerético smooth $\mu_{1}$ y el elemento sliplock $\mu_{2}$. En formato incremental, la relación se puede expresar como:

$$
d \mu=d \mu_{1}+d \mu_{2}
$$

Puesto que el elemento de degradación smooth está basado en el modelo de Wen-Bouc, la relación que establece su comportamiento histerético se puede escribir como en (5.4):

$$
d Z=d \mu_{1} \cdot \frac{\left[A-|Z|^{n}\left(\beta \operatorname{sgn}\left(d \mu_{1} Z\right)+\gamma\right)\right]}{\eta}
$$

Para definir la componente del desplazamiento $\mu_{2}$ en el elemento slip-lock se establece la siguiente relación:

$$
\begin{aligned}
& d \mu_{2}=a f(Z) d Z \\
& f(Z)=\exp \left(-\frac{Z}{Z_{s}}\right)^{2}
\end{aligned}
$$

Donde $Z_{s}$ representa una gama de valores de $Z$, en torno al valor $Z=0$, para los que ocurre el efecto slip. La variación de $f(Z)$ con respecto a $Z$ describe una gráfica similar a la de una distribución normal tal y como se muestra en la figura 19:

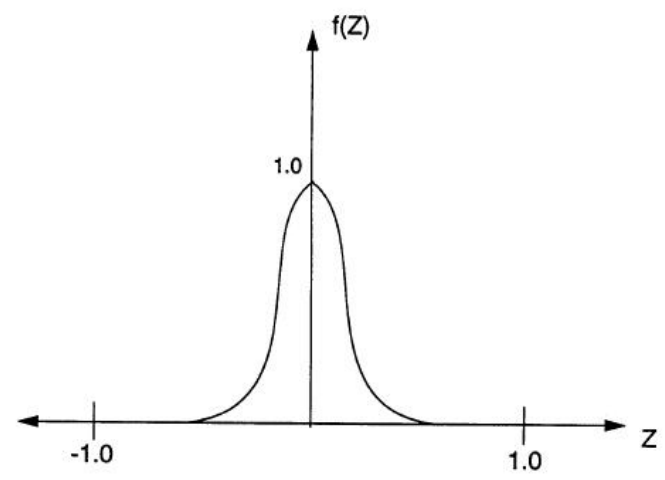

Figura 19

Sustituyendo (5.11) y (5.12) en (5.4)

Llegamos a la expresión

$$
\frac{d Z}{d \mu}=\frac{A-|Z|^{n}(\beta \operatorname{sgn}(d \mu Z)+\gamma)}{\eta\left[1+\operatorname{aexp}\left(-\frac{Z}{Z_{S}}\right)^{2}\left(A-|Z|^{n}(\beta \operatorname{sgn}(d \mu Z)-\gamma)\right]\right.}
$$

En la aplicación a este caso, se asume que la longitud de efecto slip es función de la ductilidad alcanzada:

$$
a=R_{s}\left(\mu^{r}-1\right)
$$

Donde $R_{s}$ es un parámetro de control que permite variar la longitud de efecto slip en función del tamaño de apertura de la grieta o de la armadura de refuerzo (reinforcing steel), y $\mu^{r}$ es el desplazamiento normalizado alcanzado en el ciclo de descarga previo al actual ciclo de carga o 
descarga. El efecto de variar los parámetros de control en el elemento slip-lock en la curva histerética producida por efecto pinzamiento se muestra en la Figura 20:
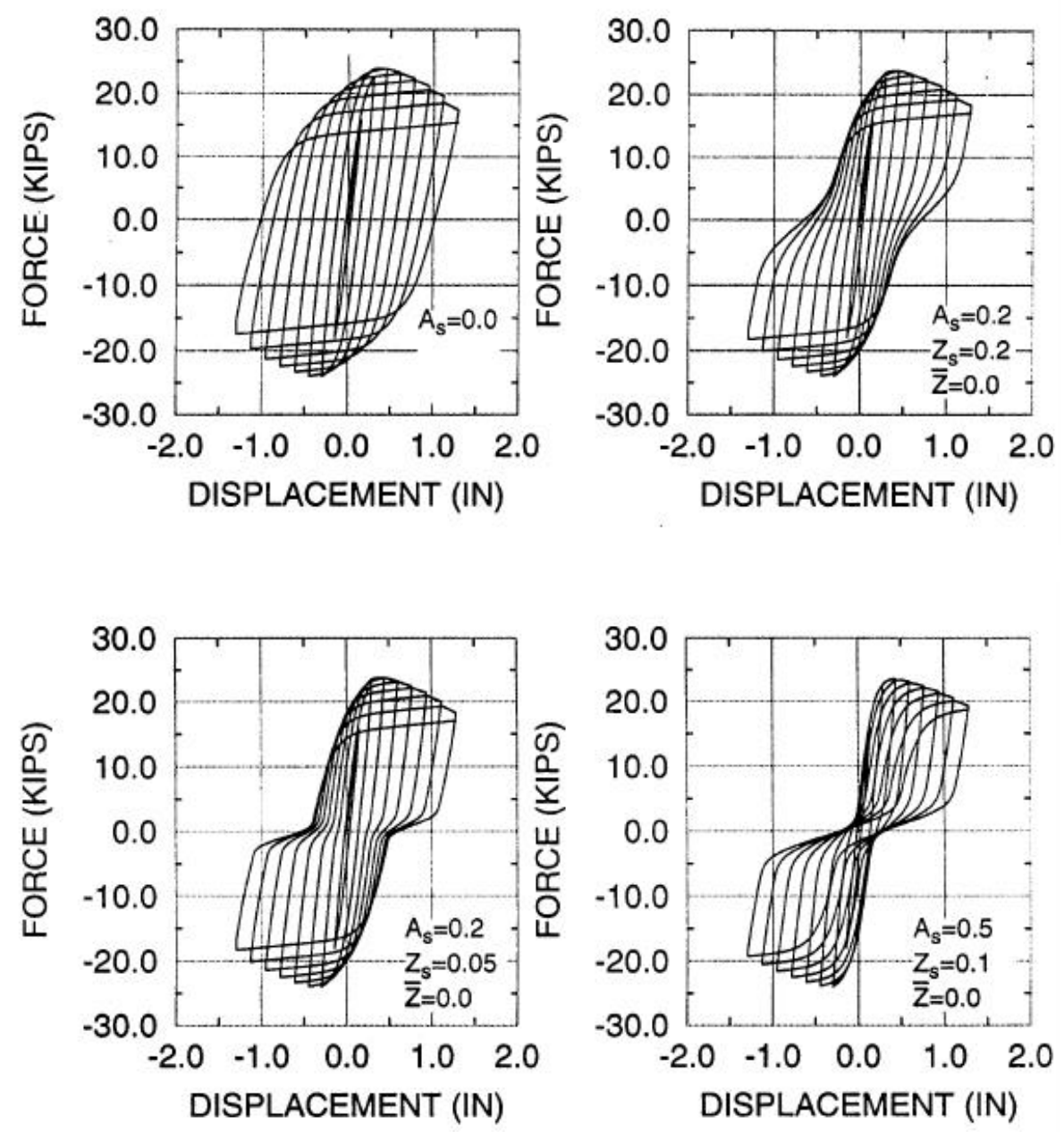

\begin{tabular}{|lcl|}
\hline \multicolumn{3}{|c|}{ Constant Parameters } \\
$A=1.0$ & $\alpha=0.01$ & $s_{k}=0.1$ \\
$\beta=0.1$ & $V_{y}=25$ Kips & $s_{p 1}=0.8$ \\
$\gamma=0.9$ & $K_{0}=125$ K/in & $s_{p 2}=1.0$ \\
$n=2$ & & $\mu_{c}=25$ \\
\hline
\end{tabular}

Figura 20

El parámetro $Z_{s}$, que controla lo acusado del efecto slip, se considera independiente de la historia de respuesta. El efecto slip ocurre cuando $Z$ es igual a $Z_{s} y$ es simétrico con respecto a $Z=0$. Para permitir que la zona efectiva de slip o deslizamiento sea simétrica con respecto a cualquier $Z=\bar{Z}$, el valor de $Z$ utilizado puede ser desplazado por el de $\bar{Z}$ :

$$
\frac{d Z}{d \mu}=\frac{A-|Z|^{n}(\beta \operatorname{sgn}(d \mu Z)+\gamma)}{\eta\left[1+\operatorname{aexp}\left(-\frac{(Z-\bar{Z})^{2}}{Z_{S}^{2}}\right)\left(A-|Z|^{n}(\beta \operatorname{sgn}(d \mu Z)-\gamma)\right)\right]}
$$

Las ecuaciones:

$$
\eta_{i}=1+s_{k}\left(\frac{\mu_{\max }^{p}+\mu_{i}}{2}\right)
$$




$$
\begin{gathered}
\mathrm{a}=\mathrm{R}_{\mathrm{s}}\left(\mu^{\mathrm{r}}-1\right) \\
\frac{d Z}{d \mu}=\frac{A-|Z|^{n}(\beta \operatorname{sgn}(d \mu Z)+\gamma)}{\eta\left[1+\operatorname{aexp}\left(-\frac{(Z-\bar{Z})^{2}}{Z_{s}^{2}}\right)\left(A-|Z|^{n}(\beta \operatorname{sgn}(d \mu Z)-\gamma)\right)\right]}
\end{gathered}
$$

proporcionan un modelo de Wen-Bouc modificado para elementos histeréticos con efecto pinzamiento, sometidos a cargas dinámicas o cuasi-estáticas. Para análisis dinámicos, la última ecuación puede escribirse como:

$$
\dot{Z}=\dot{\mu} \frac{A-|Z|^{n}(\beta \operatorname{sgn}(d \mu Z)+\gamma)}{\eta\left[1+a \cdot \exp \left(-\frac{(Z-\bar{Z})^{2}}{Z_{S}^{2}}\right)\left(A-|Z|^{n}(\beta \operatorname{sgn}(d \dot{\mu} Z)-\gamma)\right)\right]}
$$

La solución a esta ecuación diferencial puede expresarse de la siguiente forma reducida:

$$
\dot{F}(u)=f(F, u, \dot{u})
$$

Para la resolución de este tipo de ecuación diferencial se procede a la integración incremental usando el método semi-ímplicito Runge-Kutta. El incremento $\Delta \mathrm{F}$ viene dado por:

$\Delta \mathrm{F}_{\mathrm{k}}=\mathrm{F}_{\mathrm{k}+1}-\mathrm{F}_{\mathrm{k}}=\mathrm{R}_{1} \mathrm{k}_{\mathrm{k}}+\mathrm{R}_{2} \mathrm{I}_{\mathrm{k}}$

Donde el subíndice $k$ indica el paso $k$. las cantidades $k_{k} y l_{k}$ se determinan a partir de:

$$
\begin{aligned}
& k_{k}=\left[1-a_{1} \Delta x \frac{\partial f\left(F_{k}\right)}{\partial F}\right]^{-1} f\left(F_{k}\right) \Delta x \\
& l_{k}=\left[1-a_{2} \Delta x \frac{\partial f\left(F_{k}+c_{1} k_{k}\right)}{\partial F}\right]^{-1} f\left(F_{k}+b_{1} k_{k}\right) \Delta x
\end{aligned}
$$

Finalmente concluir que el comportamiento histerético de los muros de mampostería sería la suma de los tres comportamientos descritos con anterioridad, el modelo de Wen-Bouc, la degradación de rigidez y resistencia y el efecto pinzamiento. El esquema de este comportamiento se muestra en la Figura 21: 


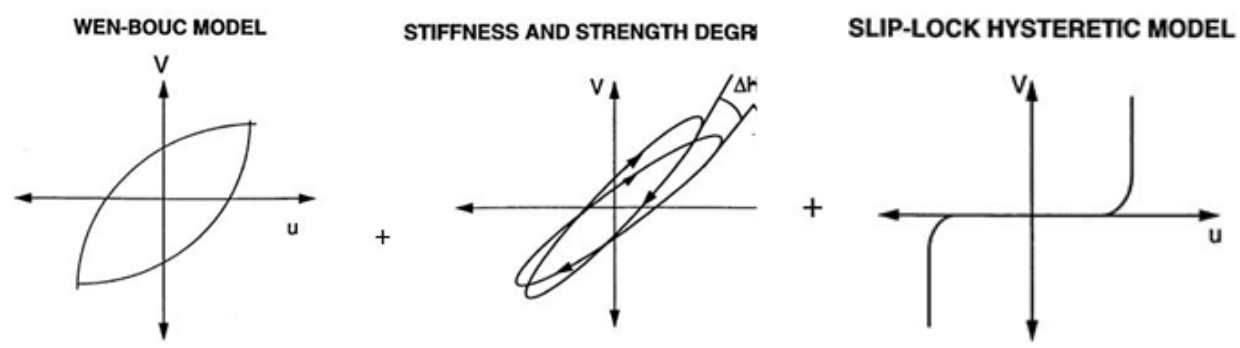

INTEGRATED MODEL IN IDARG

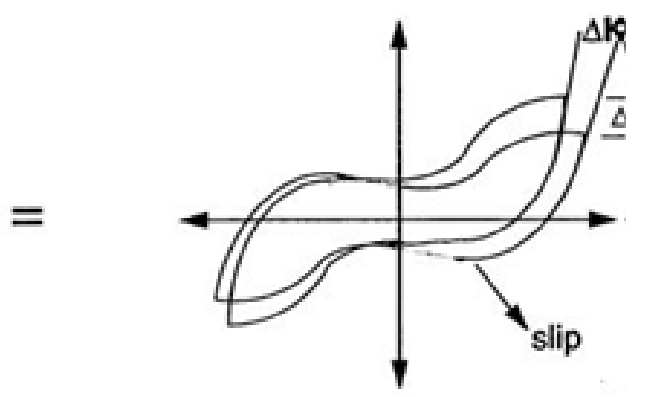

Figura 21

\section{Implementación de los elementos en el programa}

El programa Idarc permite la definición de las características mecánicas de los elementos de dos modos, (i) a través de la definición geométrica de la sección y las leyes constitutivas de los materiales, o (ii) introduciendo directamente la relación momento-curvatura, diagrama de interacción axil-momento y para el caso de los muros de fábrica de ladrillo, la rigidez inicial elástica y la resistencia última. En este estudio el modo que se ha utilizado para idealizar los pórticos es el segundo. Para el caso de vigas y columnas, la relación momento-curvatura de las secciones se ha calculado con el programa Response2000 mientras que para los muros de fábrica se han utilizado datos empíricos [REF1]

Así, las vigas han sido definidas por los siguientes parámetros:

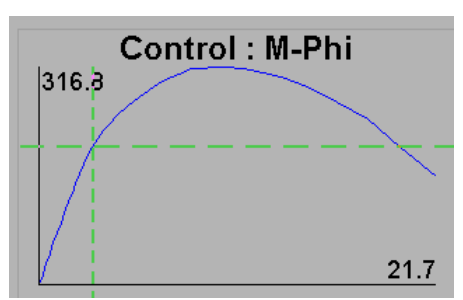

Momento de fisuración

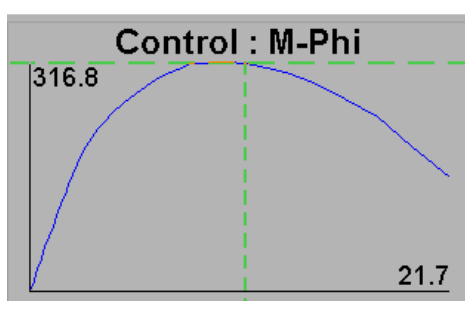

Momento y curvatura de rotura

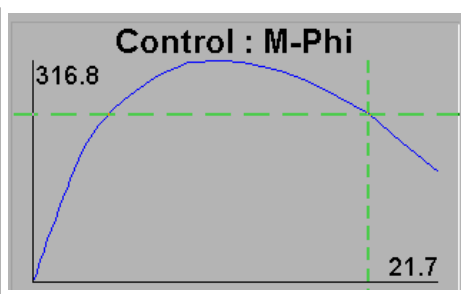

Curvatura última

La curvatura última se considera la correspondiente al 75\% del momento de rotura.

Se considera el mismo comportamiento para las cargas aplicadas de signo positivo como para las de signo negativo, el siguiente gráfico muestra el modelo trilineal con el que se han idealizado las relaciones momento-curvatura bajo cargas monótonas en el caso de vigas: 


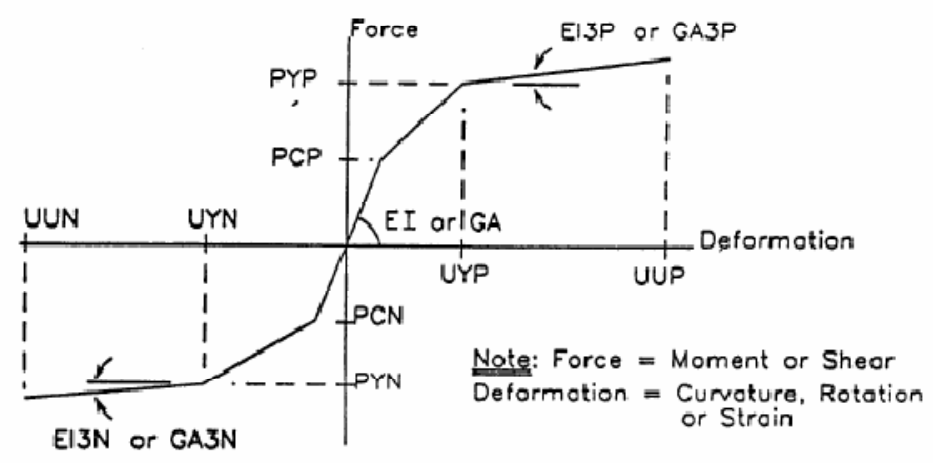

Figura 22

Para el caso de los pilares se añaden también los siguientes datos para modelizar el diagrama de interacción Axil-Momento:
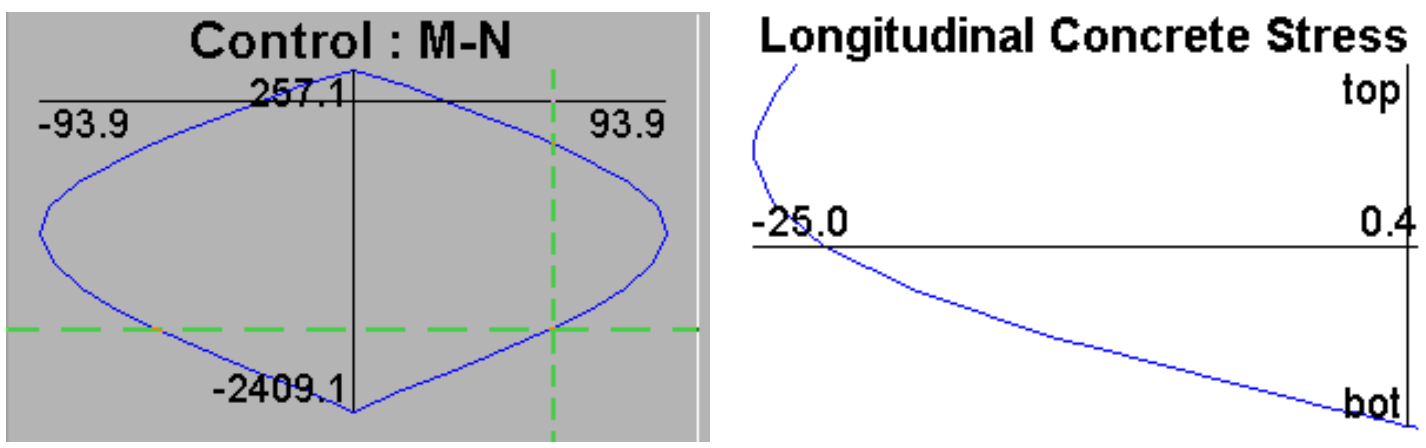

Figura 23. Axial Normal Yield

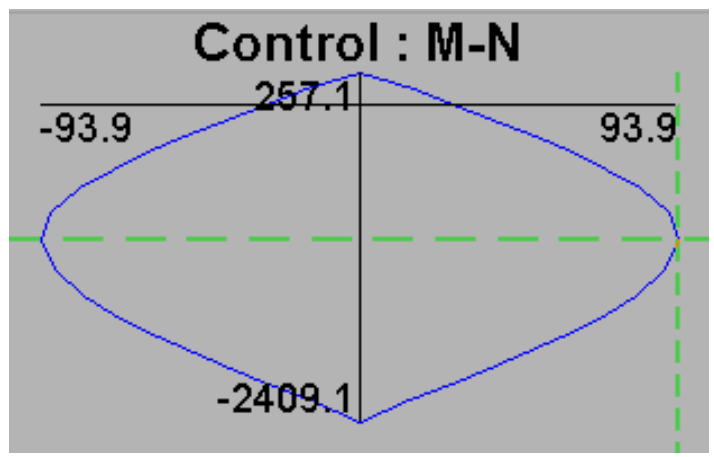

Figura 24Axial Normal Balance

A partir de los cuales, Idarc idealiza el diagrama de interacción momento-axil como se indica en la figura 25: 


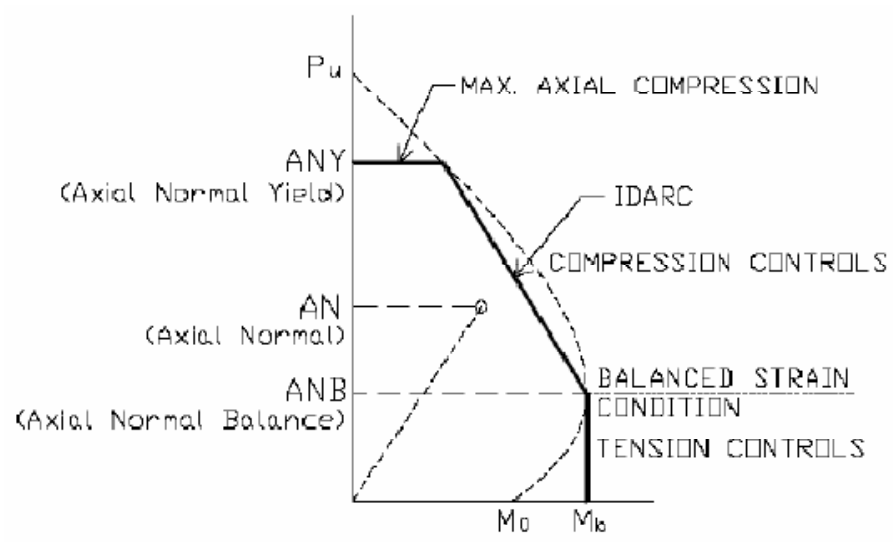

Figure P-M interaction diagram

Figura 25

En el tramo horizontal del diagrama de la figura 25 la sección no está sometida en ningún momento a esfuerzos de tracción, en el tramo vertical los esfuerzos que predominan son los de tracción y en el tramo intermedio, la compresión es el esfuerzo predominante.

Todos estos valores han sido calculados para cada uno de los elementos vigas y columnas e introducidos en el programa. Además se ha creado otro elemento ficticio, la viga biarticulada, cuyos valores de momento de fisuración y momento último son del orden de 1000 veces superiores a los introducidos para el resto de las vigas.

Por su parte para los elementos de mampostería, se han utilizado datos empíricos [REF 1], se observa en la Figura 26 los ensayos realizados en la Universidad de Purdue a partir de los cuales se obtuvieron los datos para calibrar el modelo en IDARC:

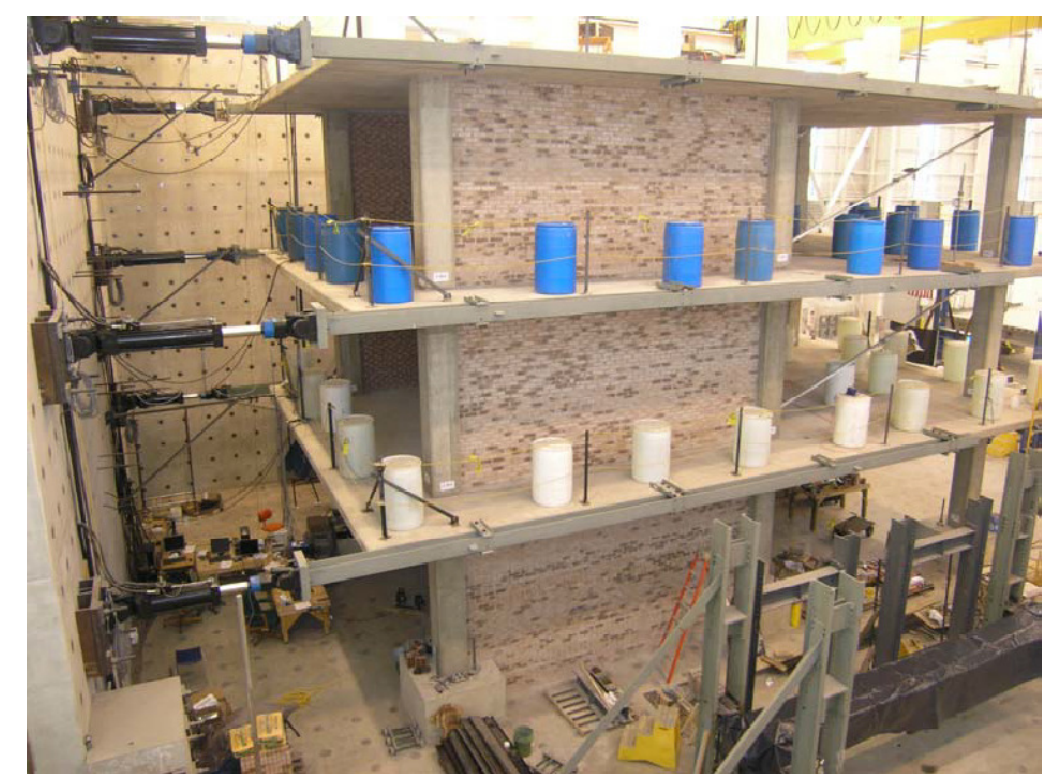

Figura 26 
Se trata de un prototipo de tres plantas a escala real, con altura entre planta de $3.05 \mathrm{~m}$ y una altura total de $9.15 \mathrm{~m}$. Los barriles simulan las cargas gravitatorias a los que estaría sometido según la normativa estadounidense.

En cuanto a la definición del comportamiento histerético de los elementos, y puesto que el trabajo que se desarrolla va enfocado a la rehabilitación sísmica de edificios dañados, se han adoptado como valores de los parámetros de control HC, HEB y HS los correspondientes a una degradación moderada de la rigidez, de la resistencia, y un efecto pinzamiento moderado. Para edificios de nueva construcción esta categoría depende del elemento y sus características constructivas. Por su parte para los muros de mampostería se han tomado los valores por defecto del programa, de acuerdo también con los ensayos experimentales [REF 1] en los que se calibraron.

\section{Implementación de los parámetros para el análisis dinámico en el programa}

El comportamiento sísmico de todos los edificios en estudio se ha obtenido mediante cálculos dinámicos directos empleando acelerogramas históricos.

Además de las cargas sísmicas se han considerado también las cargas gravitatorias lineales a las que están sometidas cada una de las vigas. La combinación de cargas sísmicas y gravitatorias utilizadas es establece el CTE para la situación sísmica, es decir:

$\sum \mathrm{G}_{\mathrm{i}}+\Psi_{2, \mathrm{i}} \sum \mathrm{Q}_{\mathrm{i}}$

siendo $\Psi_{2, i}=0.6$, coeficiente de simultaneidad, el valor de la carga $Q_{i}$ casi permanente

$\mathrm{G}_{k, j}$ son el peso propio de los elementos estructurales (vigas, columnas y forjados) y las acciones permanentes

$\mathrm{Q}_{k, 1}$ es la sobrecarga de uso para el caso de plantas distintas de la superior y la sobrecarga correspondiente a la cubierta para la planta superior

Los acelerogramas empleados en los cálculos dinámicos se han escalado a un valor prefijado de la aceleración pico horizontal. Dado que los modelos analizados son 2D, se ha empleado sólo una componente translacional horizontal del movimiento del suelo.

La fracción de amortiguamiento adoptada es del $5 \%$ respecto al crítico. EL tipo de amortiguamiento empleado es el de Rayleigh, que construye la matriz de amortiguamiento como una combinación lineal de la matriz de masa y de rigidez.

Los terremotos para los que se han evaluado los edificios son tomados de la norma japonesa y calibrados para una PGA máxima de $0.525 \mathrm{~g}$, tal y como se explica en el capítulo 6 de la memoria. 


\section{SELECCIÓN DE ACELEROGRAMAS Y CRITERIO DE ESCALADO}

El análisis del comportamiento de una estructura frente a cargas sísmicas puede estudiarse a través de cálculos dinámicos directos utilizando acelerogramas reales registrados durante terremotos históricos o sintéticos, mediante espectros elásticos de respuesta o a partir de espectros de energía introducida.

Este trabajo está basado cálculos dinámicos directos, en los cuales se aplica a cada uno de los prototipos de estructuras una serie de acelerogramas representativos. El inconveniente de este método es que el hecho de que una estructura responda bien ante la acción de un terremoto, histórico o sintético, no garantiza su buen funcionamiento ante cualquier otro que pueda ocurrir en su vida útil. La única manera de solventar esta deficiencia es someter a cada una de las estructuras a una amplia gama de terremotos o bien a algunos que sean especialmente representativos. Para investigar el comportamiento de estructuras existentes mediante cálculo dinámicos directos, en primer lugar se definen prototipos representativos y se dimensionan según la normativa aplicable a cada caso, en segundo lugar se seleccionan una serie de acelerogramas y se escalan con un determinado criterio y, finalmente se obtiene la respuesta mediante cálculos numéricos.

\section{Obtención de la aceleración de cálculo}

La forma de escalar los acelerogramas empleada en este estudio consiste en multiplicar la aceleración del registro por un factor de escala de forma que la aceleración máxima del suelo (Peak Ground Acceleration PGA) iguale un valor predeterminado. El valor de PGA que indica la aceleración máxima esperable en el suelo en un lugar determinado del territorio y para un terremoto de proyecto con un periodo de retorno determinado. En la Fig. 27 se muestra el mapa de peligrosidad sísmica mundial desarrollado en el marco del Global Seismic Hazard Assesment Program (GSHAP), donde se indica la aceleración máxima en suelo duro para una probabilidad de excedencia del $10 \%$ en 50 años, equivalente a un periodo de retorno de 500 años: 


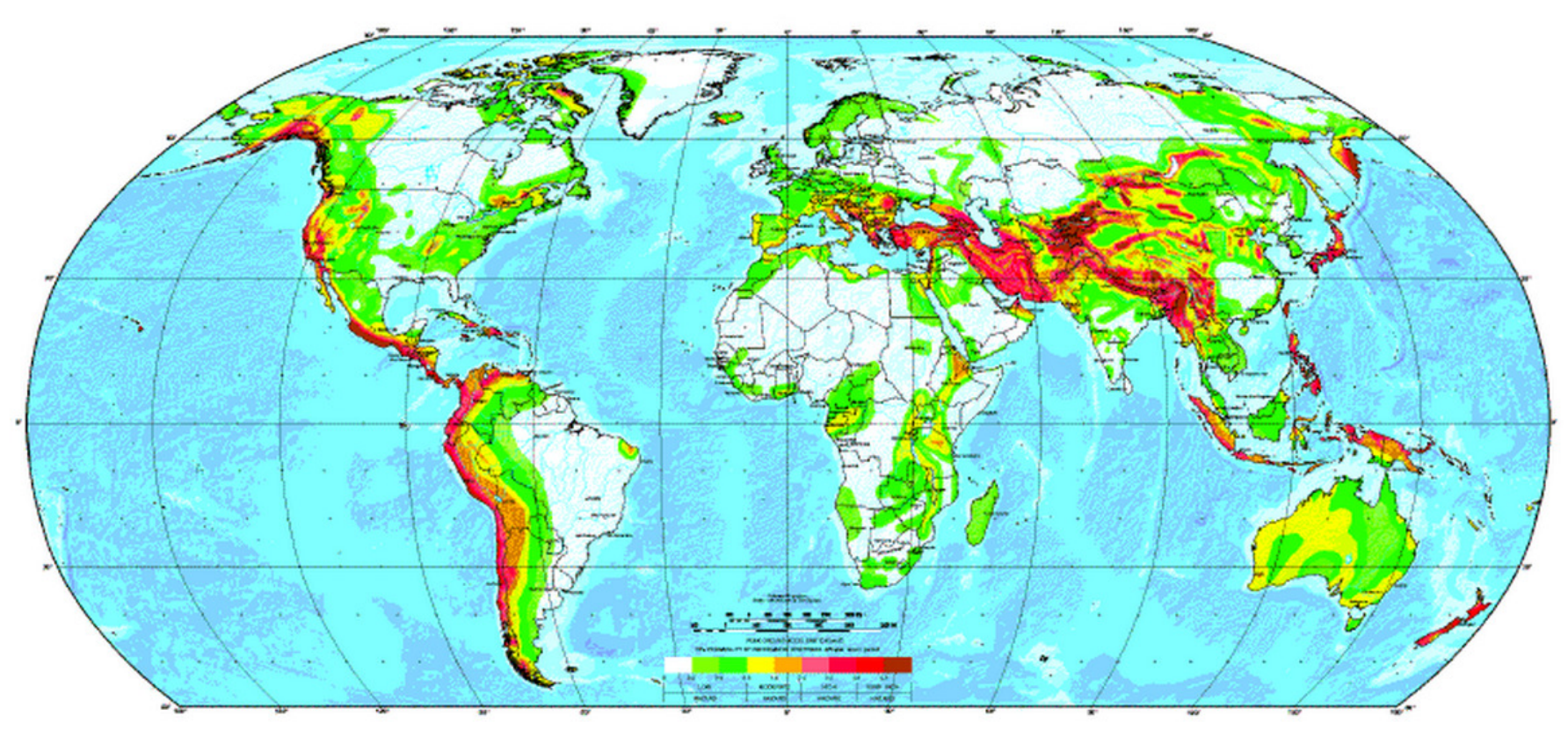

Figura 27. Marco de peligrosidad sísmica. Fuente GSAHP

$Y$ un zoom en los meridianos que delimitan el continente americano nos permite identificar la PGA para Haití y la República Dominicana, tal y como se observa en la Figura 28. 


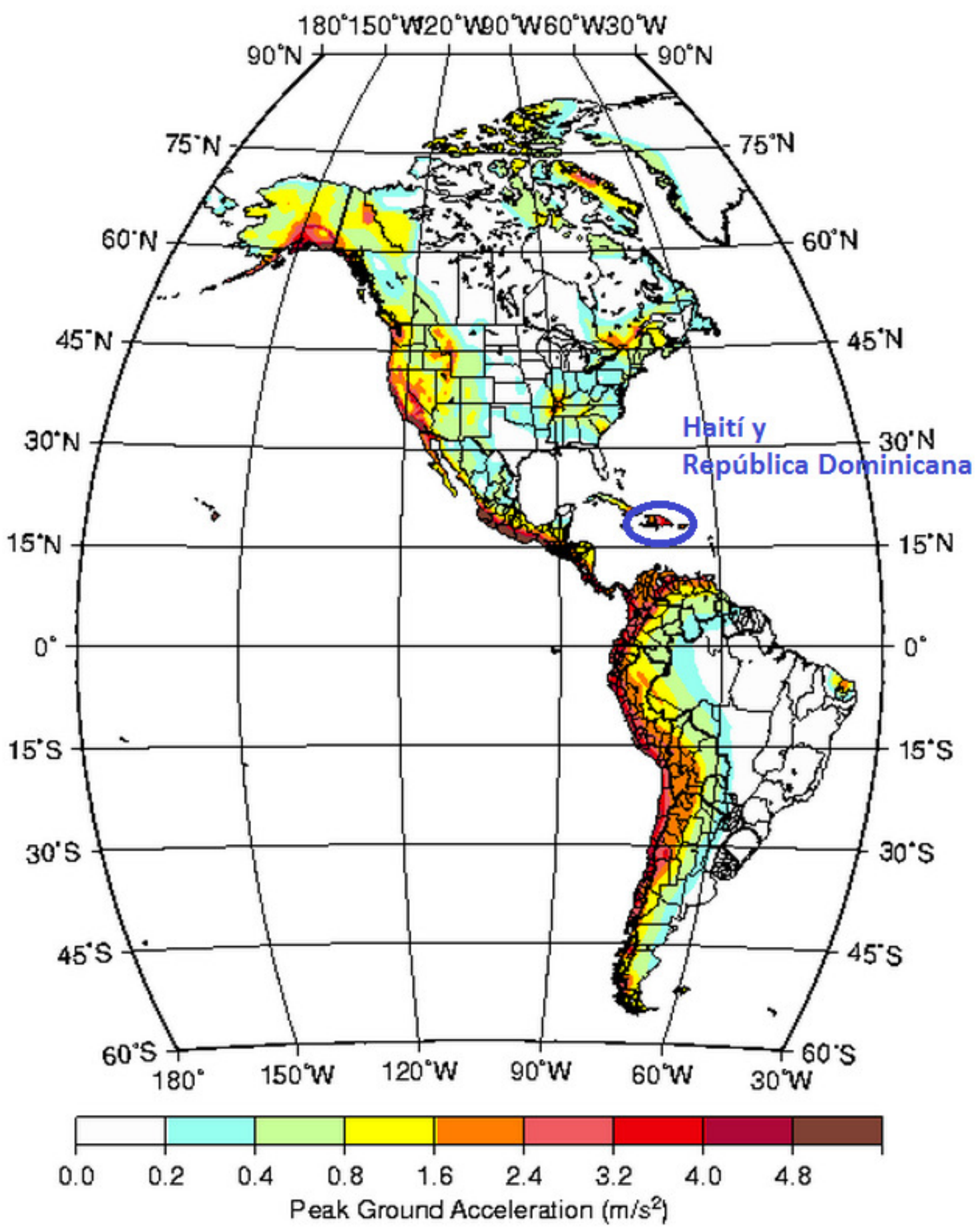

Figura 28

En el caso se Haití, este mapa de peligrosidad sísmica proporciona, para un terremoto con un periodo de retorno de 500 años, un valor de PGA entre 1.6-2.4 m/ $/ \mathrm{s}^{2}$ sobre suelo duro

A falta de una normativa sísmica en Haití, en este estudio se ha empleado el límite superior del PGA proporcionado por el mapa de peligrosidad sísmica anterior para suelo duro, es decir $2.4 \mathrm{~m} / \mathrm{s}^{2}$. Muchas de las estructuras investigadas están construidas sobre un suelo medio o blando. Del lado de la seguridad, para este estudio se ha supuesto que el suelo de cimentación 
en blando y el valor de la PGA anterior se ha corregido para tener en cuenta el tipo de suelo, aplicando las fórmulas de la normativa española NCSE-02 siguientes:

$$
a_{c}=a_{s} \cdot P \cdot S
$$

siendo $\mathrm{a}_{\mathrm{s}}$ la aceleración base, $\mathrm{P}$ un parámetro que indica la importancia del edificio y $\mathrm{S}$ el efecto de sitio.

Para $\mathrm{P}$ se ha adoptado el valor $\mathrm{P}=1$, aunque hubiese sido también razonable utilizar un valor algo mayor tratándose de edificios destinados a escuelas. A cambio, para

determinar el efecto de sitio, se ha considerado el terreno de peor calidad según la NCSE-02, que implica un valor de $\mathrm{C}=4$, y que proporciona el siguiente valor de $\mathrm{S}$ :

$$
S=\frac{C}{1.25}+3.33\left(\mathrm{P} \cdot \frac{a_{b}}{g}-0.1\right) \cdot\left(1-\frac{C}{1.25}\right)=2.14
$$

Quedando la aceleración máxima de cálculo:

$\mathrm{a}_{\mathrm{c}}=0.525 \mathrm{~g}$

\section{Elección de acelerogramas}

Dada la ausencia de registros del terremoto de Febrero de 2010 en Haití se decidido emplear tres terremotos históricos bien conocidos ampliamente empleados en cálculo de estructuras, y que son los que prescribe la norma sísmica japonesa para el cálculo de estructuras especiales: El Centro, Hachinohe y TAFT.

Cada uno de estos terremotos se caracteriza por:

- Amplitud máxima de aceleración del suelo (PGA) alcanzada

- Contenido en frecuencias, que se caracteriza por el número de veces que el acelerograma pasa por el valor cero en un segundo

- Duración del terremoto

Las figuras 29 a 31 siguientes muestran las historias de aceleración del suelo de los tres terremotos seleccionados, y la Tabla7 los valores de sus principales parámetros.

Tabla 7. Principales parámetros de los terremotos seleccionados

\begin{tabular}{|c|c|c|c|}
\hline Terremoto & Amplitud $\left(\mathrm{cm} / \mathrm{s}^{2}\right)$ & Contenido en frecuencias & Duración \\
\hline El Centro & 341.7 & 7 & 53.8 \\
\hline Hachinohe & 225 & 12 & 35.8 \\
\hline Taft & 152.7 & 10 & 53.8 \\
\hline
\end{tabular}




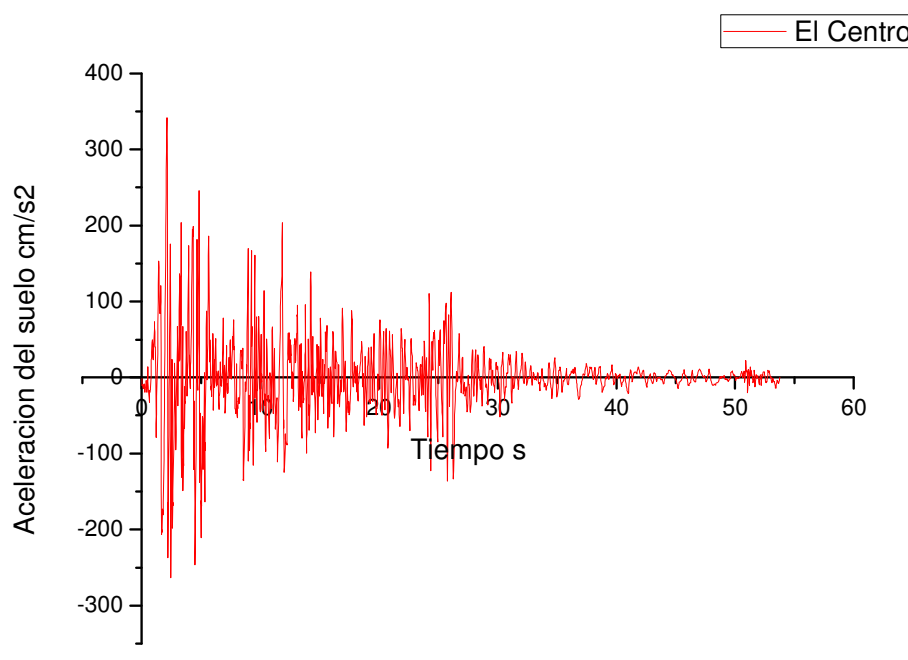

Figura 29. Acelerograma El Centro

Amplitud: $341.7 \mathrm{~cm} / \mathrm{s}^{2}$

Contenido en frecuencias: 7

Duración: $53.8 \mathrm{~s}$

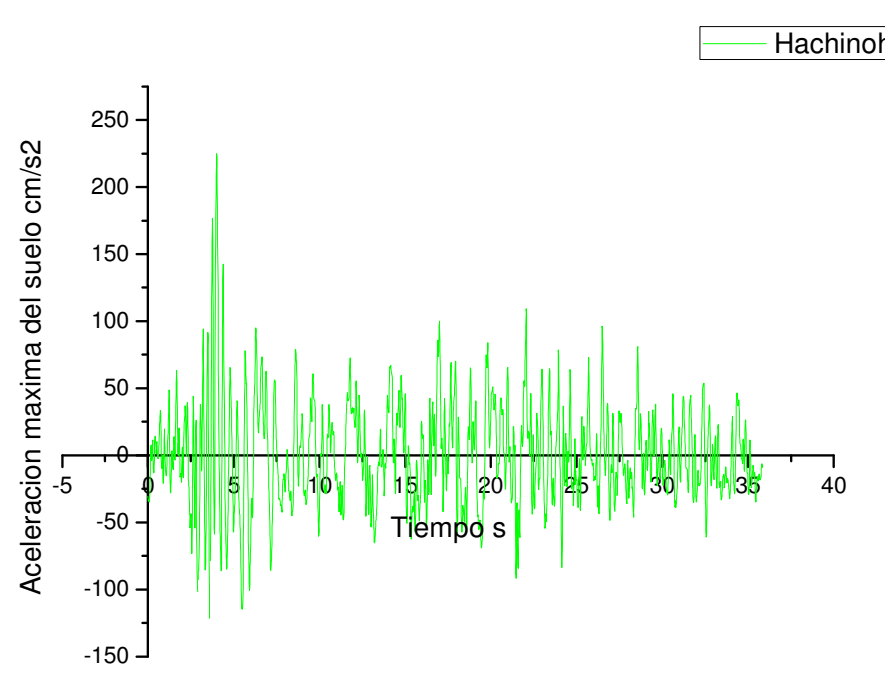

Figura 30. Acelerograma Hachinohe

Amplitud: $225 \mathrm{~cm} / \mathrm{s}^{2}$.

Contenido en frecuencias: 12

Duración: $35.8 \mathrm{~s}$ 


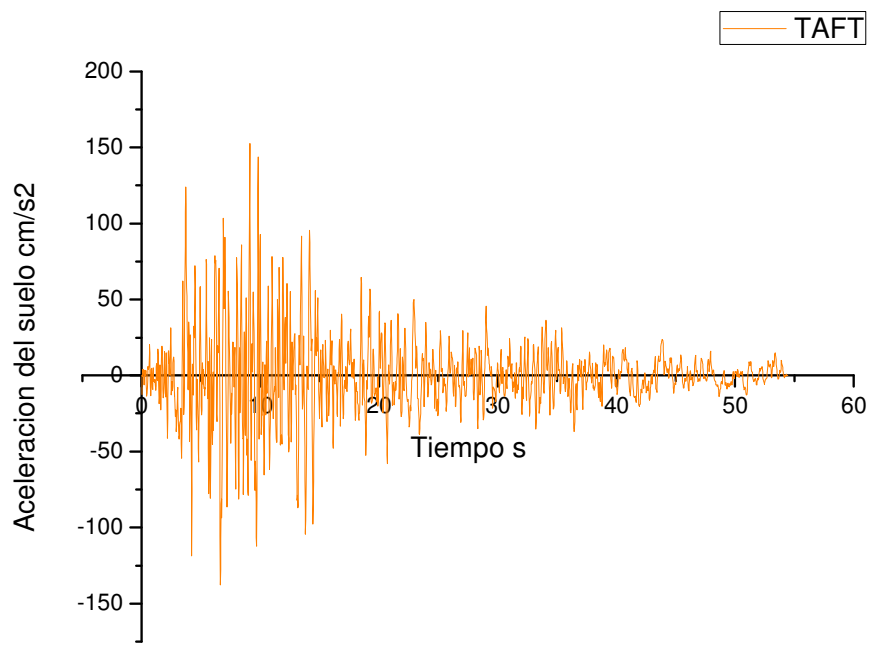

Figura 31. Acelerograma Taft

Amplitud: $152.7 \mathrm{~cm} / \mathrm{s}^{2}$

Contenido en frecuencias: 10

Duración: $54.38 \mathrm{~s}$

Los acelerogramas anteriores están sin escalar, y con ello pretende mostrar la naturaleza de éstos. Se observa que los terremotos de El Centro y TAFT son similares en cuanto a duración y contenido en frecuencias y difieren mucho en cuanto a la aceleración máxima del suelo.

Por su parte el terremoto de Hachinohe es mucho más corto, alcanza una duración intermedia entre los otros dos pero sin embargo su contenido en frecuencias es superior a ambos.

Se trata con estas diferencias entre los terremotos escogidos de cubrir la aleatoriedad de los efectos sísmicos en cada edificio.

A continuación, en las figuras 32 a 34 se muestran los espectros elásticos de respuesta en términos de aceleración absoluta y los espectros de energía introducida expresada en forma de velocidad equivalente Ve [REF. 13 ecuación 1.16], de estos tres terremotos. Estos espectros representan la respuesta de sistemas de un grado de libertad y amortiguamiento del $5 \%$ con respecto al amortiguamiento crítico. La velocidad equivalente se define como la raíz cuadrada de dos veces la energía introducida dividida por la masa total del sistema. Para la obtención de los espectros de respuesta se ha escalado cada uno de los acelerogramas a la PGA de cálculo. 


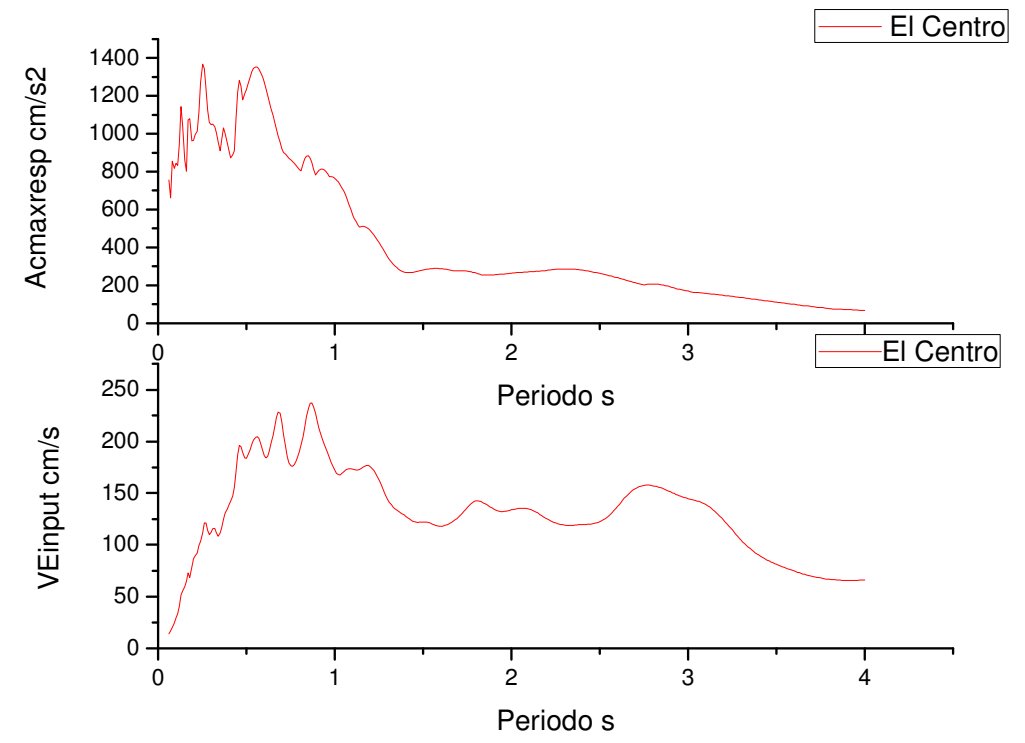

Ilustración 32. Espectro elástico de respuesta El Centro

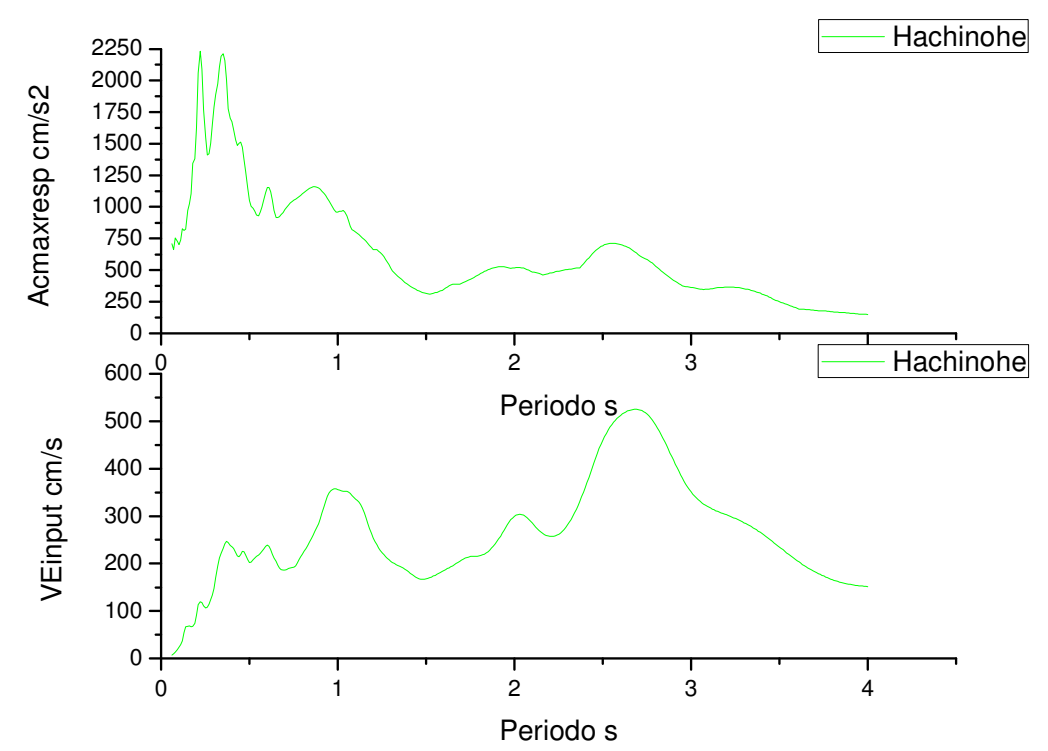

Ilustración 33. Espectro elástico de respuesta Hachinohe 


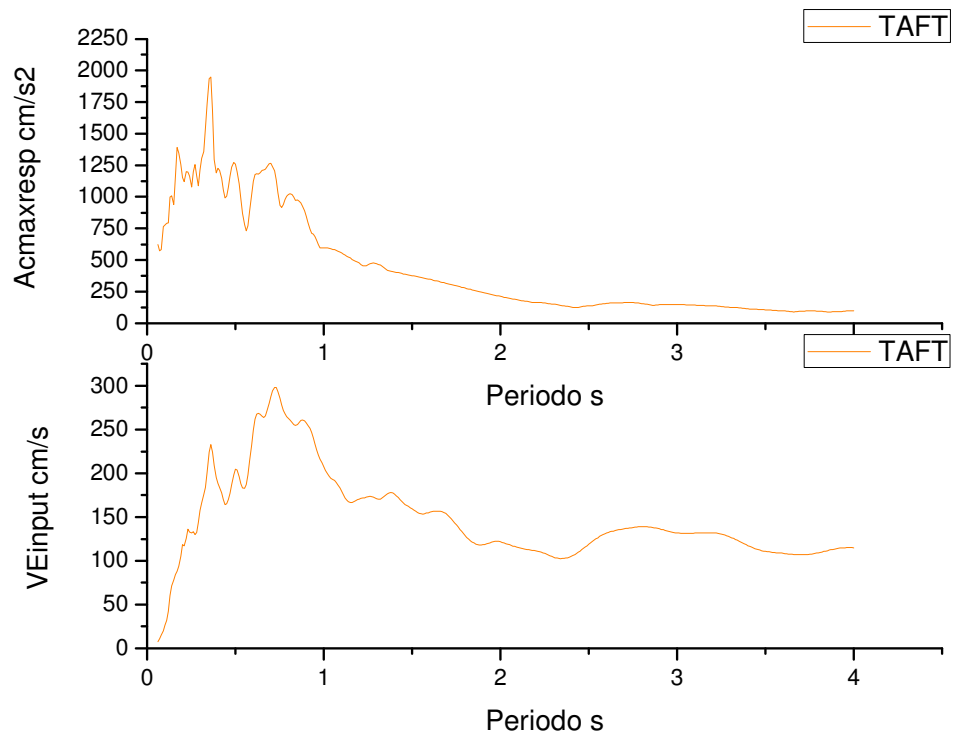

Ilustración 34. Espectro elástico de respuesta Taft

Al comparar los espectros elásticos de respuesta se observan la diferencia notable entre estos tres terremotos. Los prototipos objeto de este trabajo están en un rango de periodos bajos, entre 0.2 y 0.6 segundos donde se dan fuertes variaciones en los espectros, lo cual nos conducirá previsiblemente a resultados muy diferentes para cada uno de ellos. 


\section{CONFIGURACIONES DE MUROS EN LOS PÓRTICOS}

\section{Configuraciones de prototipos con muros de fábrica de ladrillo analizados}

Las configuraciones posibles para la ubicación de los muros de fábrica de ladrillo en los prototipos definidos en capítulos anteriores deben respetar las hipótesis mencionadas en el capítulo 5:

- Unión rígida entre pórticos en serie en un plano vertical: No se consideran los efectos de los muros de ladrillo perpendiculares a la dirección del sismo

- Empotramiento perfecto de los pilares en el arranque

- Simetría del edificio en la dirección del sismo: garantiza la ausencia de efectos de torsión e implica la ubicación de los muros de ladrillo en el centro y por parejas a ambos lados del eje de simetría

Además las configuraciones posibles deben respetar los usos arquitectónicos para los que está proyectado el edificio (uso escolar). Por tanto, los criterios seguidos para distribuir los muros en planta han sido: (i) salvo que sea estrictamente necesario no se colocarán muros de fábrica de ladrillo en dos pórticos continuos; (ii) se empezará siempre con el pórtico del centro si el número de pórticos es impar, y a continuación por los pórticos de los extremos alternativamente hasta llegar al centro.

Las configuraciones de rellenos de muros en los pórticos se han considerado adecuadas cuando el índice de daño global de Park \& Ang es inferior a 1 en al menos dos terremotos. Es decir, cuando la estructura con esa configuración de muros no colapsa para dos de los tres terremotos investigados. A efectos de denominación de los prototipos con distintas configuraciones de muros, se mantendrá el nombre inicial del prototipo añadiendo un término relativo al número de muros añadidos. Así, al prototipo inicial sin muros se añade al final del nombre del prototipo las letras M0; cuando se rellena con muros el vano central (caso de que el número de pórticos es impar) o cuando se rellenan los dos vanos centrales (caso de que el número de pórticos sea par) se denota como M1. Así se van rellenando sucesivamente vanos de los pórticos con muros de ladrillo. .

Así para el caso H01-2 (Fig. 35) obtendríamos todas las posibilidades de relleno de vanos que se ilustran en la Fig. 36:

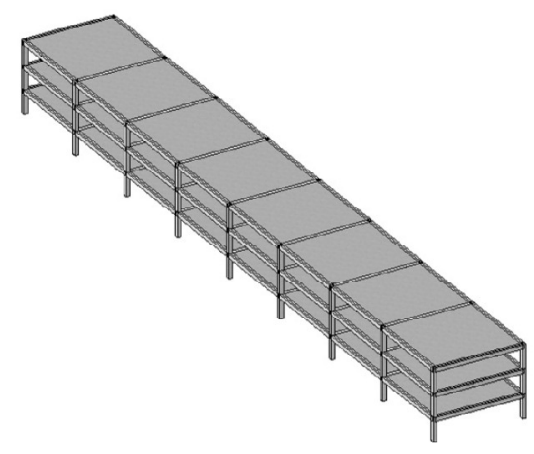

Figura 35 

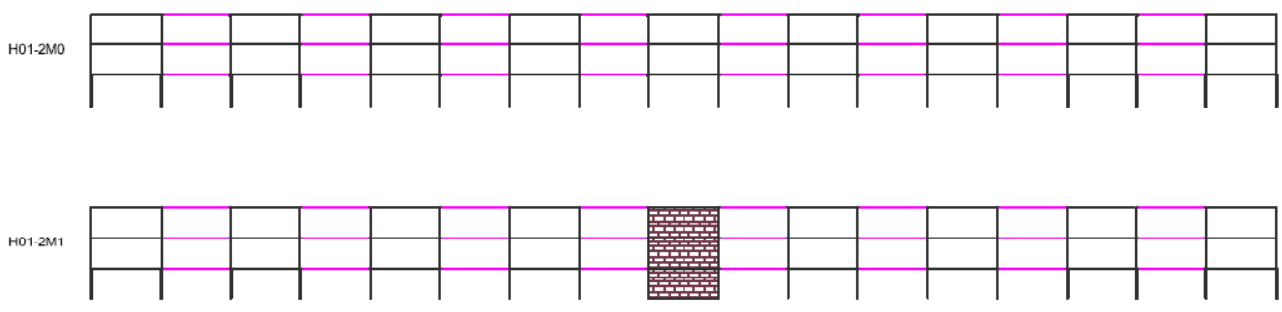

H01-2N2

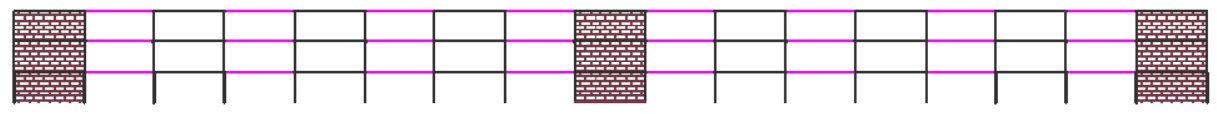

H01-2N3

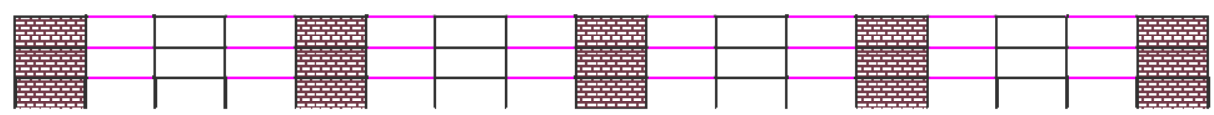

H01-2M4

El Centro 2.075

Hachinohe 2.870

TAFT 0.604

H01-2M5

El Centro 0.531

Hachinohe 1.287

TAFT 0.639
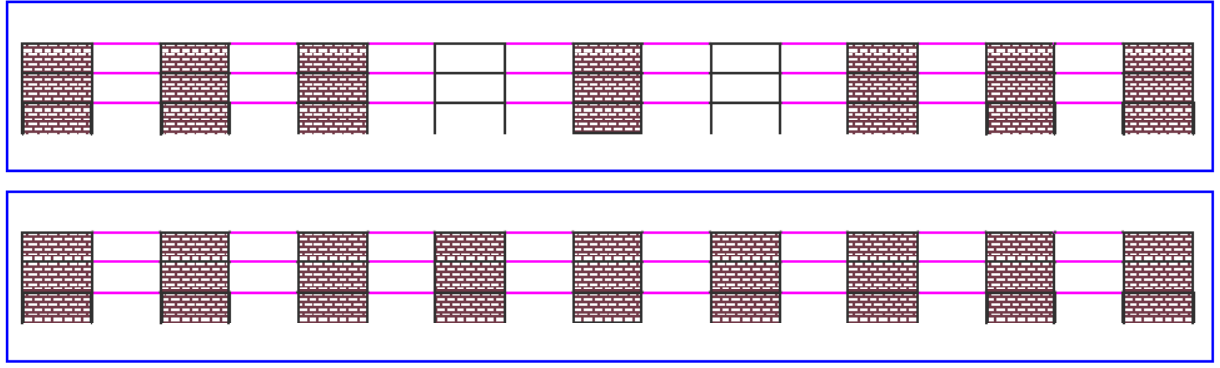

Figura 36

Como se ha indicado anteriormente, de todas las posibilidades de relleno de vanos posibles, sólo se muestran en este trabajo las configuraciones para las cuales el índice de daño de Park y Ang en el pórtico en al menos dos de los tres terremotos era inferior a 1. Un índice de daño superior a 1 significa colapso total del pórtico.

Las dos últimas configuraciones son las que se estudian para este edificio.

Para cada una de estas estructuras se ha calculado el periodo de vibración para poder ubicarlas en una región del espectro de respuesta elástico de cada terremoto.

Para el cálculo del periodo de cada edificio se ha realizado un análisis bajo cargas laterales monótamente crecientes para determinar las rigideces laterales de cada planta. El programa IDARC no permite realizar directamente este tipo de análisis cuando hay muros de fábrica, por lo que se ha recurrido al artificio de realizar un cálculo dinámico directo no lineal, asignando a la estructura un amortiguamiento nulo, y empleando un terremoto ficticio, cuya aceleración se va incrementando en $0.01 \mathrm{~cm} / \mathrm{s}^{2}$ cada $0.02 \mathrm{~s}$ hasta llegar a una aceleración de $1 \mathrm{~cm} / \mathrm{s}^{2}$.

A partir de los datos de rigidez y de masa de cada planta se ha procedido al cálculo del periodo mediante un programa de cálculo dinámico para modelos de masas concentradas llamado LUMPST. El periodo $T_{0}$ que proporciona este programa es el que tendría la estructura en régimen elástico. Para tener en cuenta que cuando la estructura plastifica el periodo se alarga, 
$T_{0}$ se ha corregido mediante la formulación que se muestra a continuación propuesta por Akiyama[REF. 13]

La ecuación del movimiento de un sistema elástico con amortiguamiento sometido a cargas dinámicas se puede escribir como:

$$
M \ddot{y}+C \dot{y}+F(y)=F_{e}
$$

Donde:

M masa

$C \dot{y}$ fuerza de amortiguamiento

$F(y)$ fuerza restauradora

$F_{e}$ fuerza sísmica(=-M $\left.\ddot{z}_{0}\right)$

$\ddot{z}_{0}$ movimiento horizontal del suelo

y desplazamiento horizontal relativo de la masa respecto del suelo

$$
\begin{gathered}
\text { Si denominamos } \quad \mathrm{s} \quad R=-M\left(\ddot{z}_{0}+\ddot{y}\right) \\
R=C \dot{y}+F(y)
\end{gathered}
$$

En el caso de que $\mathrm{C}=0$ (sistema elástico sin amortiguamiento), la relación entre $\mathrm{R}$ e y sería una recta,

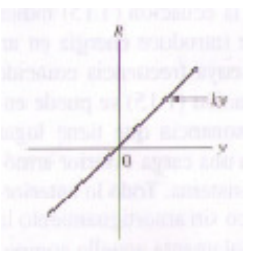

Figura 37. Sistema elástico sin amortiguamiento

Sin embargo, a medida que aumenta la constante $C$, la relación entre $\mathrm{R}$ e y se aleja de ser una recta

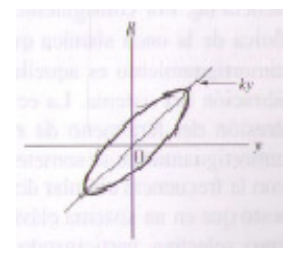

Figura 38. Sistema elástico con amortiguamiento

La ecuación del movimiento en vibración libre queda:

$\ddot{y}+2 h w_{0} \dot{y}+w_{0}^{2}=0$, 
Siendo $h=\frac{C}{2 M w_{0}}$ la fracción de amortiguamiento respecto al crítico

Suponiendo que las condiciones iniciales son $y=y_{0}$ e $\dot{y}=0$ la ecuación que expresa el movimiento en vibración libre quedará de la siguiente manera:

$y=\frac{y_{0}}{\sqrt{1-h^{2}}} e^{-h w_{0} t} \cos \left(\sqrt{1-h^{2}} w_{0} t-\varepsilon\right) ; \tan ^{-1} \varepsilon=\frac{h}{\sqrt{1-h^{2}}}$

Y el periodo de vibración libre vale

$$
T=\frac{2 \pi}{\sqrt{1-h^{2}}} \sqrt{\frac{M}{k}}
$$

En las estructuras convencionales, la fracción de amortiguamiento suele ser muy baja, y por tanto el periodo de vibración dado por (7.6) es muy próximo al correspondiente a una vibración libre elástica sin amortiguamiento, $2 \pi \sqrt{M / k}$. No ocurre lo mismo con la rigidez tangencial, $k_{t}$ representada por la pendiente de la recta tangente a la curva R-y en la figura 4, cuyo valor fluctúa de forma significativa alrededor de la rigidez $\mathrm{k}$ que es la pendiente de la recta que pasa por el origen en dicha figura. Definiendo un periodo instantáneo de vibración $T_{t}$ como:

$$
T_{t}=2 \pi \sqrt{\frac{M}{k_{t}}}
$$

El periodo $T_{t}$ está dentro del siguiente intervalo:

$T_{0}-\Delta T \leq T_{t} \leq T_{0}+\Delta T$

Siendo $\Delta \mathrm{T}$ el margen de fluctuación de $\mathrm{T}_{\mathrm{t}}$ que crece al aumentar $\mathrm{C}(\mathrm{o} \mathrm{h}) . \Delta \mathrm{T}$ y $\mathrm{h}$ se pueden relacionar de forma aproximada mediante la siguiente ecuación

$\Delta \mathrm{T}=1.5 \mathrm{hT}_{0}$

Se concluye que un sistema elástico con amortiguamiento se puede interpretar como un sistema elástico sin amortiguamiento con una gama de períodos dados por la ecuación 7.8. De esta manera, resulta razonable pensar que la existencia de amortiguamiento en un sistema dinámico lo que hace es aumentar el número de componentes armónicas del movimiento del suelo que suministran energía al sistema. Por ello, suponiendo que las componentes armónicas introducen energía sísmica de forma uniforme, el input de energía se puede estimar promediando como sigue:

$$
E=\frac{M \int_{T_{0}-\Delta T}^{T_{0}+\Delta T}{ }_{0} V_{E}(T)^{2} d T}{2 \cdot 2 \Delta T}
$$

$Y$ en el caso de que se tratase de un sistema elástico sin amortiguamiento, la variación del periodo vendría dada por la pérdida de rigidez, siendo únicamente posible su incremento. Para este sistema, el periodo instantáneo de vibración del sistema se encontraría en el intervalo:

$T_{0} \leq T_{t} \leq T_{0}+\Delta T$ 
Y el input de energía se podría expresar como en el caso de un sistema elástico con amortiguamiento como:

$$
E=\frac{M \int_{T_{0}}^{T_{0}+\Delta T}{ }_{0} V_{E}(T)^{2} d T}{2 \cdot 2 \Delta T}
$$

Por otra parte, a medida que crece el amortiguamiento (o valor de $h$ ) es espectro de input de energía se suaviza, pudiendo distinguir entre dos regiones:

$$
\begin{array}{ll}
V_{E}=a T=\frac{V_{E m}}{T_{G}} T & \mathrm{~T}_{0}<\mathrm{T}_{\mathrm{G}} \\
\mathrm{V}_{\mathrm{E}}=\mathrm{V}_{\mathrm{Em}} & \mathrm{T}_{0}>\mathrm{T}_{\mathrm{G}}
\end{array}
$$

$T_{G}$ periodo de vibración frontera entre dominios

$V_{E m}$ valor máximo del espectro de energía expresada en términos de pseudo velocidad equivalente

Llamando $T_{m}$ al valor máximo del periodo instantáneo de vibración del sistema elastoplástico, $\mathrm{T}_{\mathrm{m}}=\mathrm{T}_{0}+\Delta \mathrm{T}$, aplicando la ecuación 7.13 en 7.12 se obtiene:

$E=\frac{M}{2}\left(a \sqrt{\frac{T_{0}^{2}+T_{0} T_{m}+T_{m}^{2}}{3}}\right)^{2}=\frac{M\left(a T_{e}\right)^{2}}{2}$

Siendo

$T_{e}=\sqrt{\frac{T_{0}^{2}+T_{0} T_{m}+T_{m}^{2}}{3}}$

Siendo:

$\mathrm{T}_{0}$ el periodo de vibración inicial

$T_{e}$ el periodo efectivo de vibración,

$T_{m}$ el valor máximo del periodo instantáneo de vibración del sistema elastoplástico, $T_{m}=T_{0}+\Delta T$

Puesto que en el trabajo que se desarrolla se ha utilizado un valor de $h=0.05$, llegamos a que:

Así se obtiene que $\mathrm{T}_{\mathrm{e}}=1.256 \mathrm{~T}_{0}$.

Tabla 8. Resumen periodos iniciales y finales de las combinaciones analizadas

\begin{tabular}{|l|l|l|l|l|l|l|l|l|}
\hline Prototipo & $\mathrm{H} 01-1$ & $\mathrm{H} 01-2$ & $\mathrm{H} 02-1$ & $\mathrm{H} 02-2$ & $\mathrm{H} 03-1$ & $\mathrm{H} 03-2$ & $\mathrm{H} 04-1$ & $\mathrm{H} 04-2$ \\
\hline $\mathrm{T}_{0}(\mathrm{~s})$ & 0.5346 & 0.7976 & 0.3486 & 0.5097 & 0.3922 & 0.5743 & 0.3627 & 0.5214 \\
\hline Combinación & $\mathrm{H} 01-1 \mathrm{M} 5$ & $\mathrm{H} 01-2 \mathrm{M} 4$ & $\mathrm{H} 02-1 \mathrm{M} 2$ & $\mathrm{H} 02-2 \mathrm{M} 2$ & $\mathrm{H} 03-1 \mathrm{M} 2$ & $\mathrm{H} 03-2 \mathrm{M} 2$ & $\mathrm{H} 04-1 \mathrm{M} 2$ & $\mathrm{H} 04-2 \mathrm{M} 3$ \\
\hline $\mathrm{T}_{\mathrm{e}}(\mathrm{s})$ & 0.2766 & 0.4645 & 0.2289 & 0.4233 & 0.2807 & 0.4171 & 0.2787 & 0.2848 \\
\hline Combinación & $\mathrm{H} 01-1 \mathrm{M} 6$ & $\mathrm{H} 01-2 \mathrm{M} 5$ & $\mathrm{H} 02-1 \mathrm{M} 3$ & $\mathrm{H} 02-2 \mathrm{M} 3$ & $\mathrm{H} 03-1 \mathrm{M} 5$ & $\mathrm{H} 03-2 \mathrm{M} 3$ & $\mathrm{H} 04-1 \mathrm{M} 3$ & $\mathrm{H} 04-2 \mathrm{M} 4$ \\
\hline $\mathrm{T}_{\mathrm{e}}(\mathrm{s})$ & 0.2886 & 0.3906 & 0.192 & 0.2926 & 0.1959 & 0.3510 & 0.2384 & 0.2616 \\
\hline Combinación & $\mathrm{H} 01-1 \mathrm{M} 7$ & & & $\mathrm{H} 02-2 \mathrm{M} 4-2$ & & H03-2M4 & & \\
\hline $\mathrm{T}_{\mathrm{e}}(\mathrm{s})$ & 0.3043 & & & 0.3177 & & 0.3158 & & \\
\hline
\end{tabular}


En las Fig. 39 a 73 se representan los espectros elásticos de respuesta de los terremotos utilizados y con líneas verticales los periodos $T_{e}$ obtenidos para cada modelo analizado. Para mayor claridad, junto a los espectros se muestran también las configuraciones de muros y modelos a los que se hace referencia en las leyendas. 


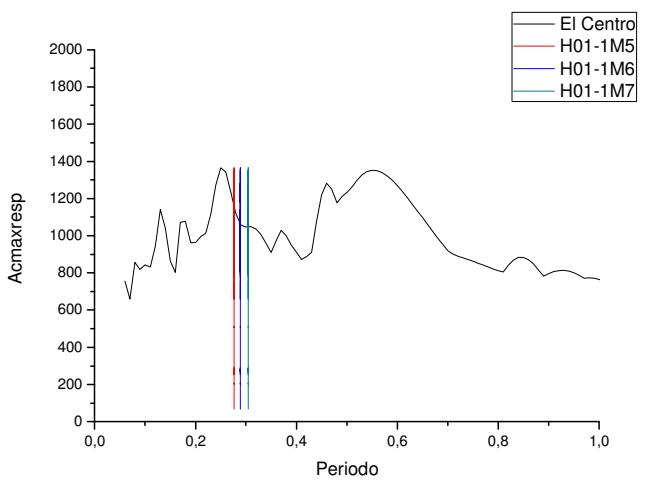

(a)

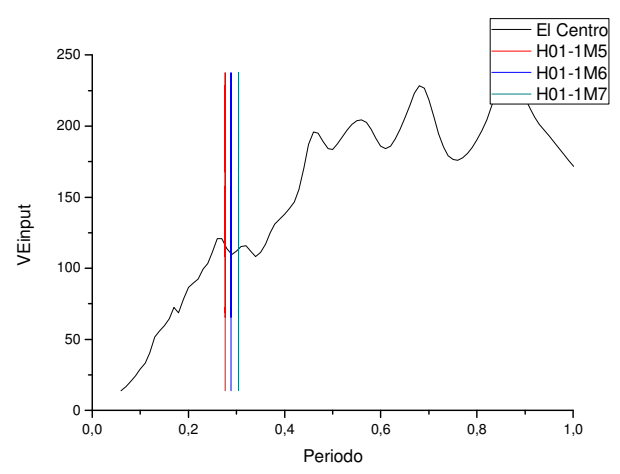

(b)

Figura 39. Espectro de respuesta elástico. El Centro. H01-1

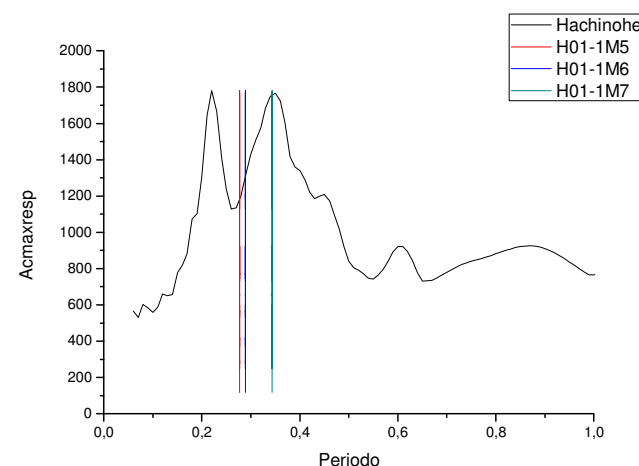

(5a)

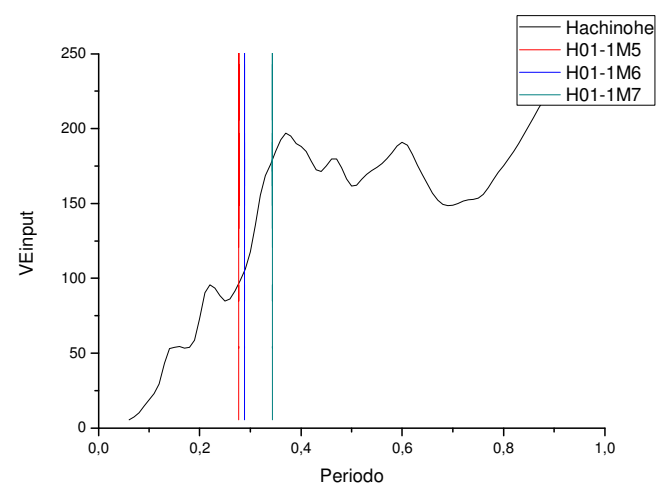

(5b)

Figura 40. Espectro elástico de respuesta. Hachinohe. H01-1

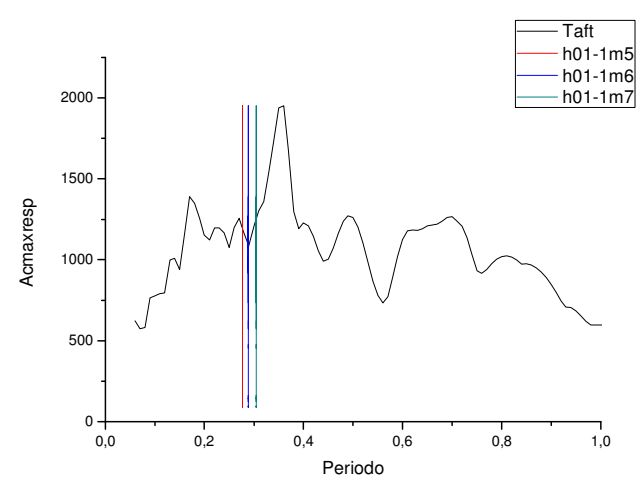

(6a)

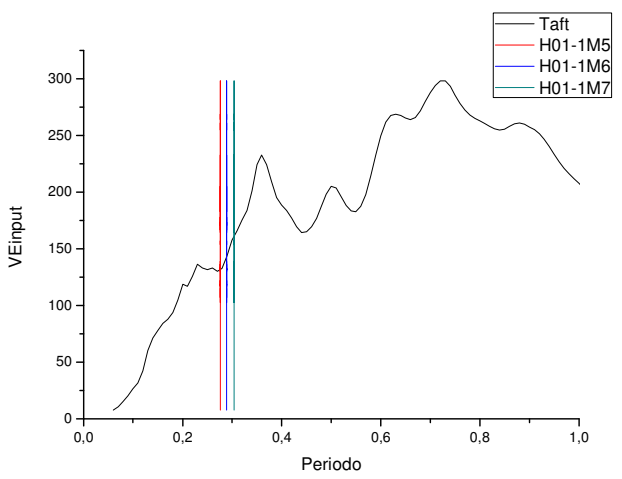

(6b)

Figura 41. Espectro de respuesta elástico. Taft. H01-1 
H01-1M5

El Centro 0.337

Hachinohe 0.753

Taft 1.015

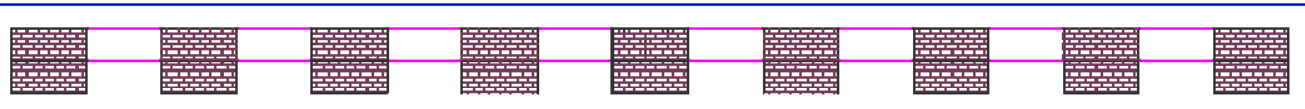

H01-1M6

El Centro 0.331

Hachinohe 1.369

Taft 0.948

\begin{tabular}{|c|c|c|c|c|c|c|c|c|}
\hline 覽虽蜀 & 覽虽睪 & 覽虽蜸 & & 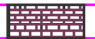 & & 覽虽 & 覽虽峑 & 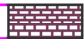 \\
\hline 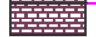 & 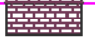 & 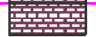 & 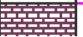 & 㯺覽 & 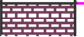 & 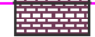 & 㯺啈 & 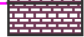 \\
\hline
\end{tabular}

H01-1M7

El Centro 1.496

Hachinohe 1.606

\begin{tabular}{|c|c|c|c|c|c|c|c|c|}
\hline 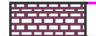 & 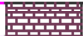 & 覽喃 & & 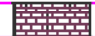 & & 覽堸 & 覽呚罢 & 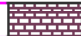 \\
\hline 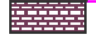 & & 覽覽 & 㯺聏䖯 & 籜監 & 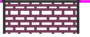 & 鑒垔 & & 覽聖蒂 \\
\hline
\end{tabular}

Taft 1.005

Figura 42. Modelo para IDARC de los prototipos e índices de daño

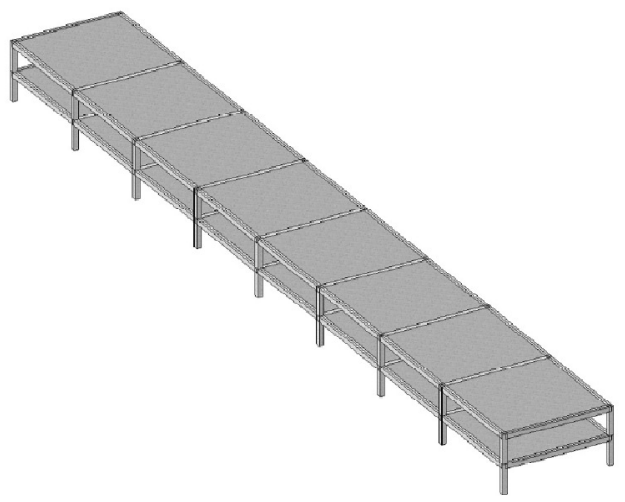

Figura 43. Prototipo H01-1

Se utilizarán para el análisis del prototipo H01-1 los resultados de las combinaciones correspondientes a:

- H01-1M5

- H01-1M6

En ellos se cumple el requisito de que el índice de daño de Park\&Ang es inferior a 1 para al menos dos terremotos. 


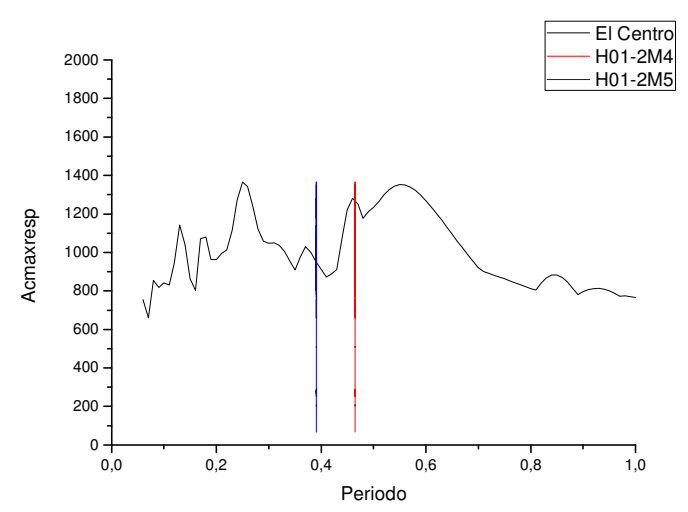

(a)

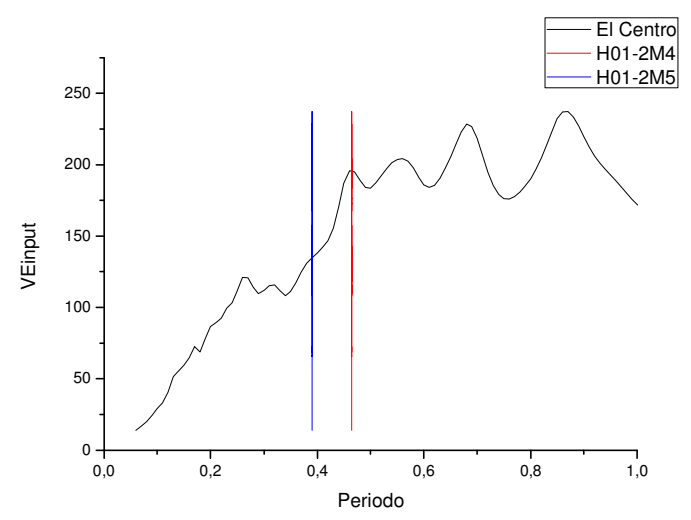

(b)

Figura 44. Espectro elástico de respuesta. El Centro. H01-2

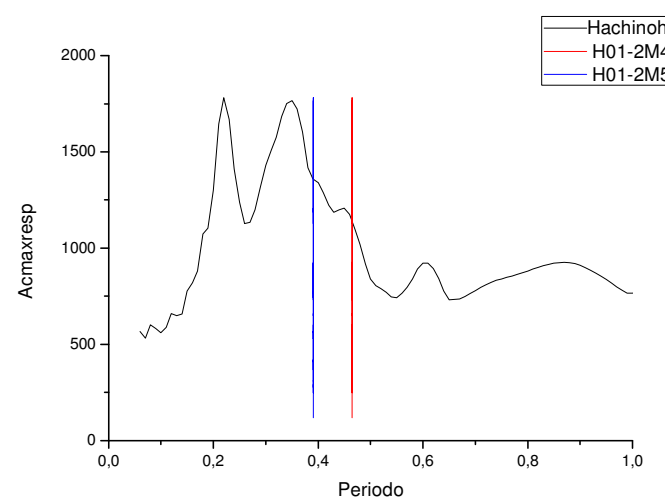

(a)

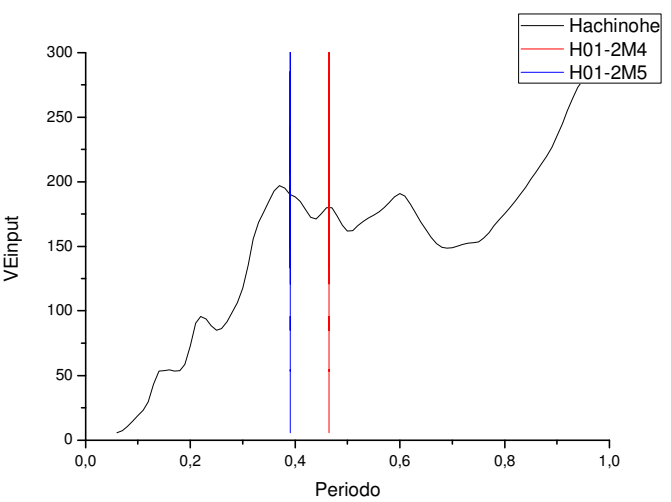

(b)

Figura 45. Espectro de respuesta elástico. Hachinohe. H01-2

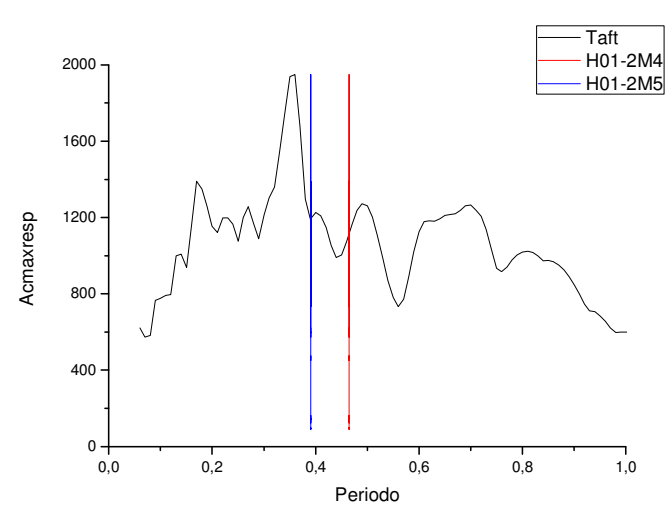

(a)

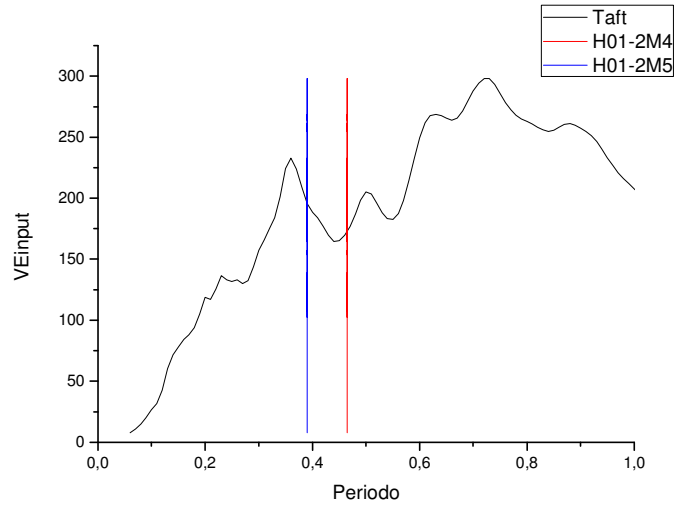

(b)

Figura 46. Espectro elástico de respuesta. Taft. H01-2 
$\mathrm{H} 01-2 \mathrm{M} 4$

El Centro 2.075

Hachinohe 2.870

TAFT 0.604

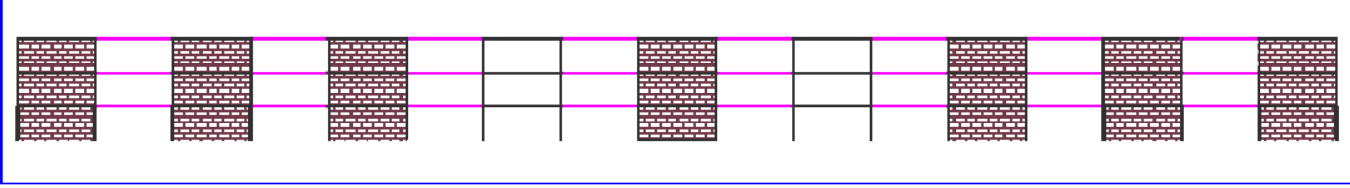

H01-2M5

El Centro 0.531

Hachinohe 1.287

TAFT 0.639

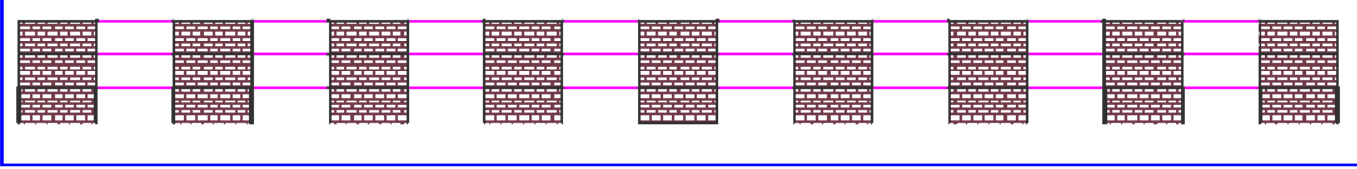

Figura 47. Modelo de los prototipos para IDARC y resultado del índice de daño

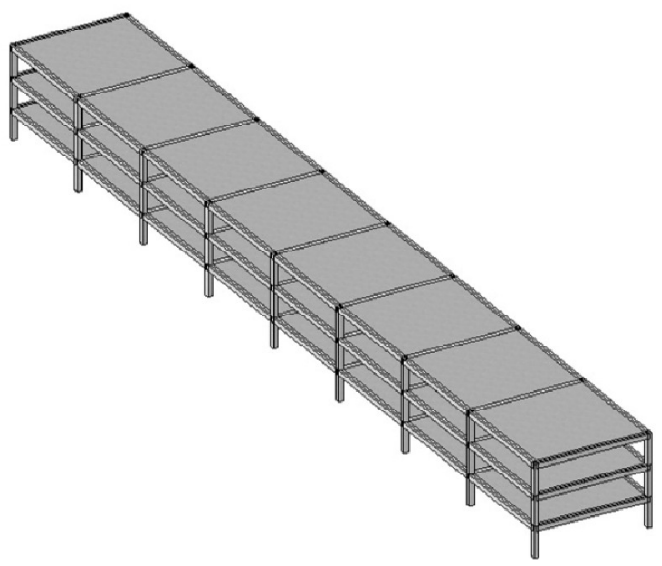

Figura 48. Prototipo H01-2

Se utilizarán para el análisis del prototipo H01-2 los resultados de las combinaciones correspondientes a:

- H01-2M4: Solamente los datos desprendidos del terremoto TAFT, ya que se observa que el input de energía es el mismo para los tres terremotos, sin embargo la componente de los desplazamientos laterales es más fuerte para los terremotos de El Centro y Hachinohe, lo que hace que sus índices de daño alcancen valores más elevados. Por esta razón los resultados pueden resultar engañosos. Sin embargo el terremoto de TAFT si es representativo ya que, como se ha mencionado, el input de energía equivalente es similar para los tres casos $\left(V_{e}=200 \mathrm{~cm} / \mathrm{s}\right)$ y su índice de daño es bastante inferior a 1 (0.604).

- H01-2M5: Ya que cumple el requisito de que, al menos para dos terremotos, el índice de daño de Park\&Ang es inferior a 1. 


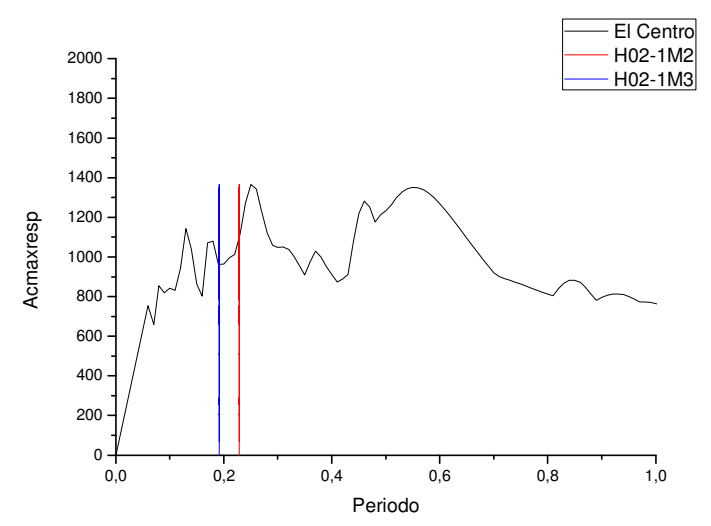

(a)

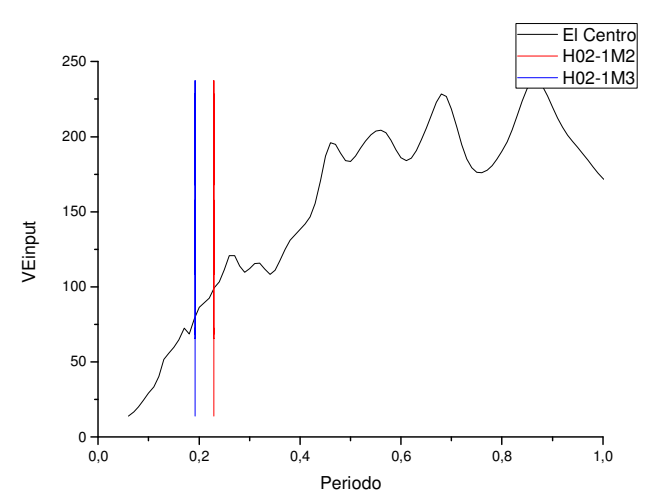

(b)

Figura 49. Espectro elástico de respuesta. El Centro. H02-1

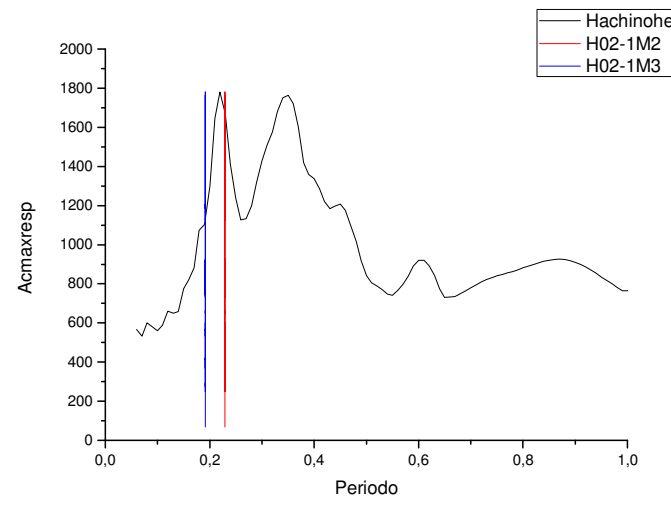

(a)

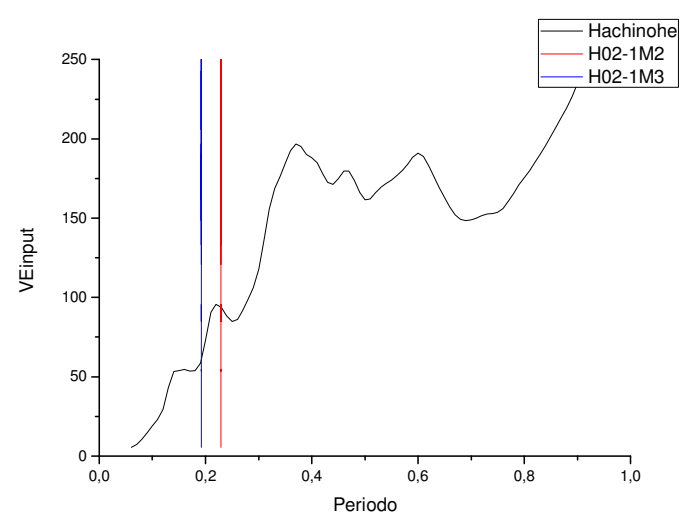

(b)

Figura 50. Espectro elástico de respuesta. Hachinohe. H02-1

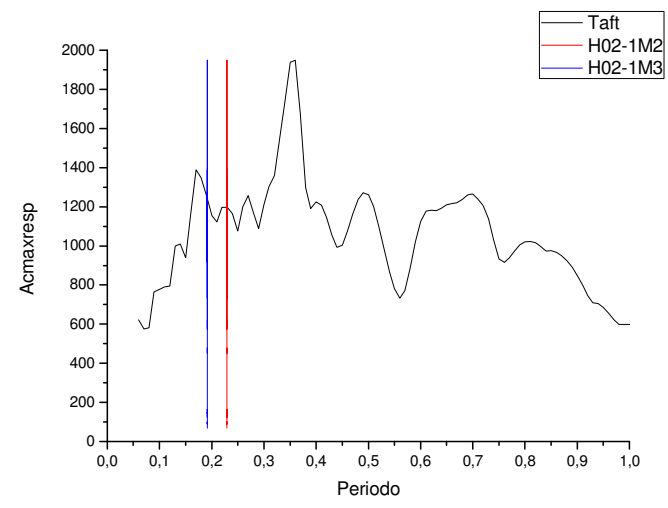

(a)

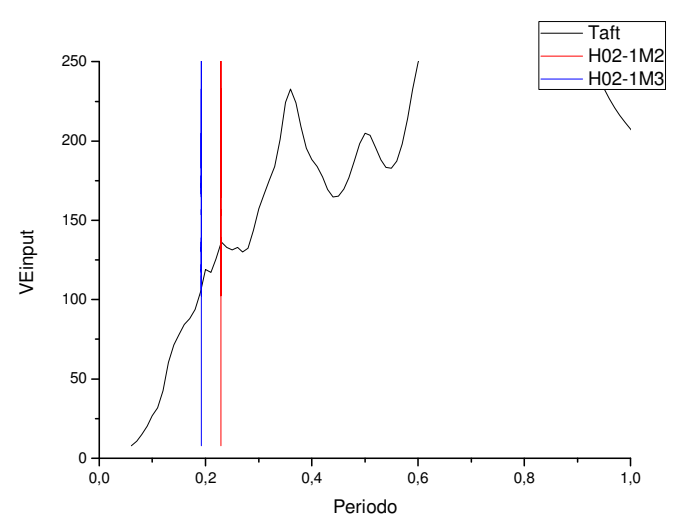

(b)

Figura 51. Espectro elástico de respuesta. Taft. H02-1 
$\mathrm{H} 02-1 \mathrm{M} 2$

EI Centro 0.246

Hachinohe 0.537

Taft 0.581

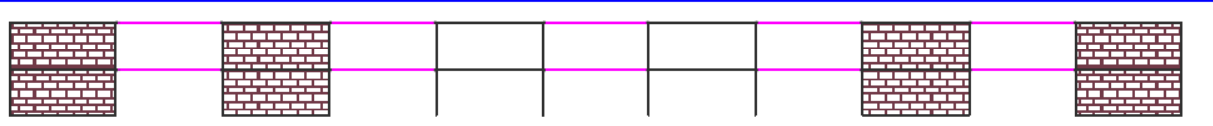

$\mathrm{H} 02-1 \mathrm{M} 3$

El Centro 0.176

Hachinohe 71.224

Taft 0.406

Figura 52. Modelo de los prototipos para IDARC y resultado del índice de daño

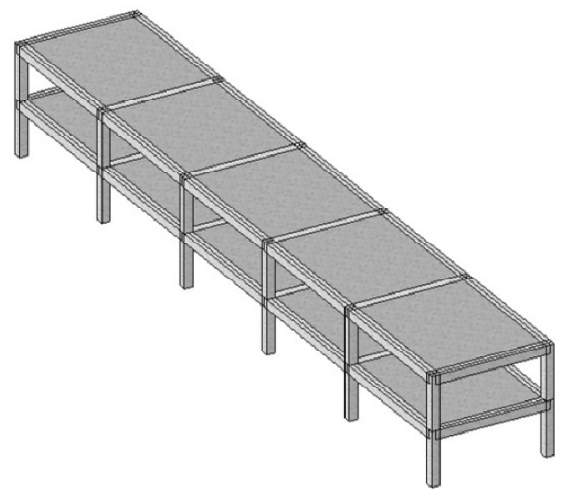

Figura 53. Prototipo H02-1

Se utilizarán para el análisis del prototipo H02-1 los resultados de las combinaciones correspondientes a:

- H02-1M2: Ya que el índice de daño de Park\&Ang al menos para dos terremotos es inferior a 1

- H02-1M3: Ya que el índice de daño de Park\&Ang al menos para dos terremotos es inferior a 1

NOTA: Tras los ensayos dinámicos directos, se desprende de los resultados de IDARC el siguiente gráfico (ejemplo utilizado H02-1M3):

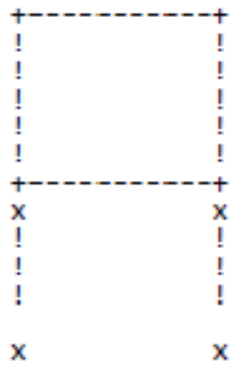

Se observa que para un índice de daño de 0.176 , en los pórticos de la planta baja ha comenzado a romper el hormigón (símbolo x). Si mostramos además el mismo prototipo, para una combinación que sí ha colapsado (H02-1M1) 
Universidad de Granada-Trabajo Fin de Máster

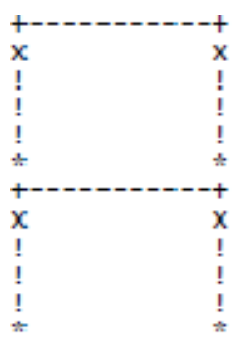

Se observa que igualmente ha comenzado a romper el hormigón y se ha producido fallo en los pilares (símbolo*), lo cual conduce al colapso del edificio. La concentración de daño es, para todos los casos analizados en este estudio, en los pilares casi exclusivamente. 


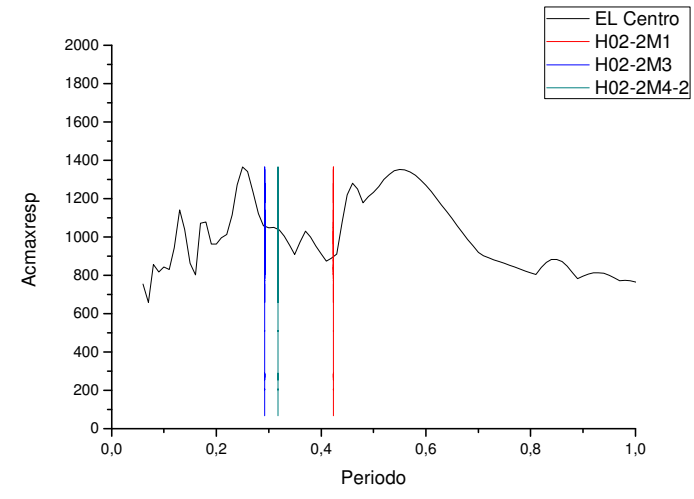

(a)

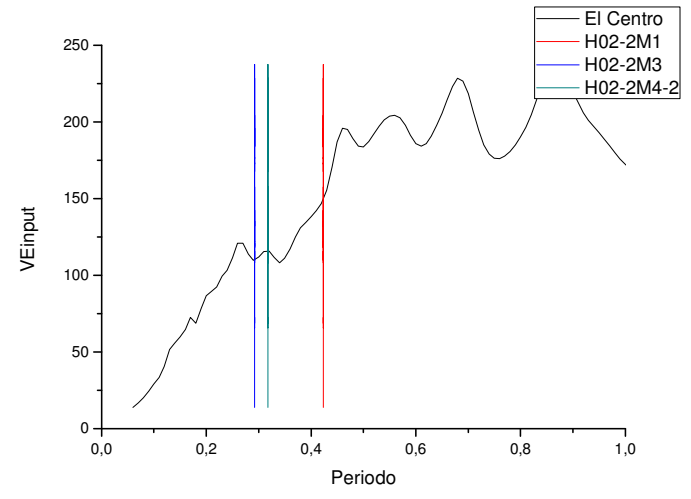

(b)

Figura 54. Espectro elástico de respuesta. El Centro. H02-2

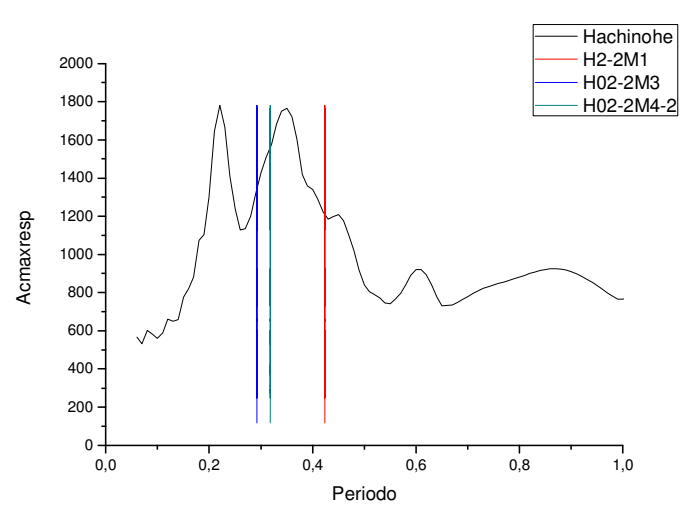

(a)

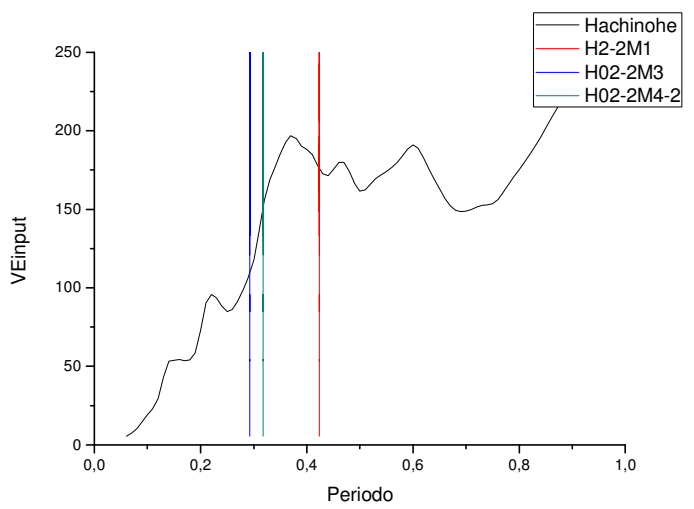

(b)

Figura 55. Espectro elástico de respuesta. Hachinohe. H02-2

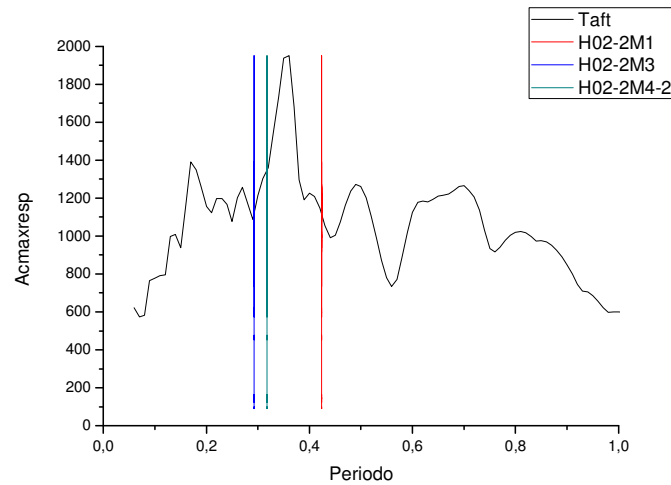

(a)

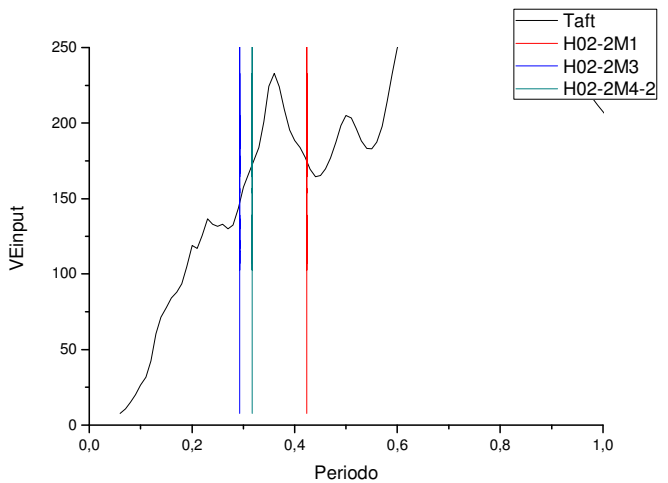

(b)

Figura 56. Espectro elástico de respuesta. Taft. H02-2 
$\mathrm{H} 02-2 \mathrm{M} 1$

EI Centro 2.796

Hachinohe 6.818

TAFT 0.690

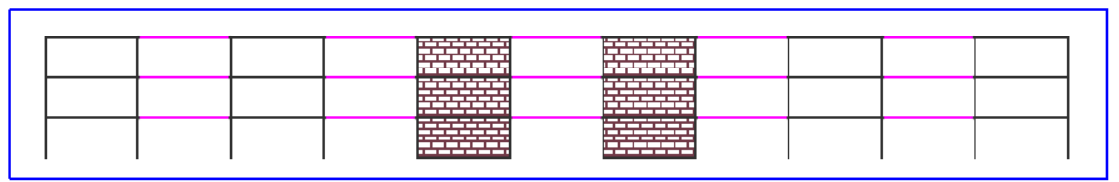

$\mathrm{H} 02-2 \mathrm{M} 3$

El Centro 0.875

Hachinohe 1.488

TAFT 3.330

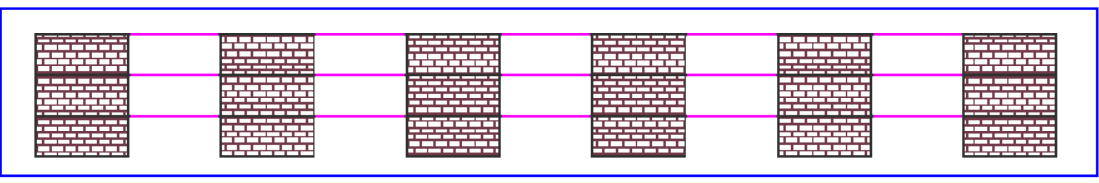

$\mathrm{H} 02-2 \mathrm{M} 4-2$

El Centro 0.363

Hachinohe 2.030

TAFT 0.572

\begin{tabular}{|c|c|c|c|c|c|}
\hline 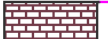 & & 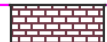 & 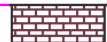 & & 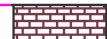 \\
\hline 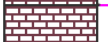 & & 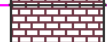 & 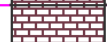 & & 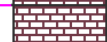 \\
\hline 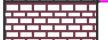 & 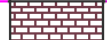 & 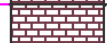 & 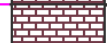 & 䁂 & 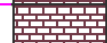 \\
\hline
\end{tabular}

Figura 57. Modelo de prototipos para IDARC y resultado del índice de daño

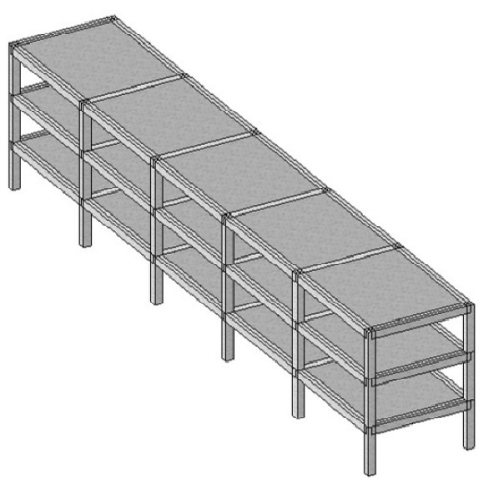

Figura 58. Prototipo H02-2

Para este caso no se considera representativo ninguno de las tres combinaciones presentadas, ya que:

- $\quad$ H02-2M2 y H02-2M3: En ningún caso cumplen el requisito de que el índice de daño de Park\&Ang sea inferior a 1 para al menos dos terremotos.

- H02-2M4-2: La eliminación de los muros de las plantas superiores de los pórticos 2 y 5 traslada el periodo del edificio a una zona del espectro a caballo entre las otras dos configuraciones, pero no hay ningún indicio de que esta configuración sea más estable que las otras dos, no puede generalizar por tanto el comportamiento de este edificio concreto. 


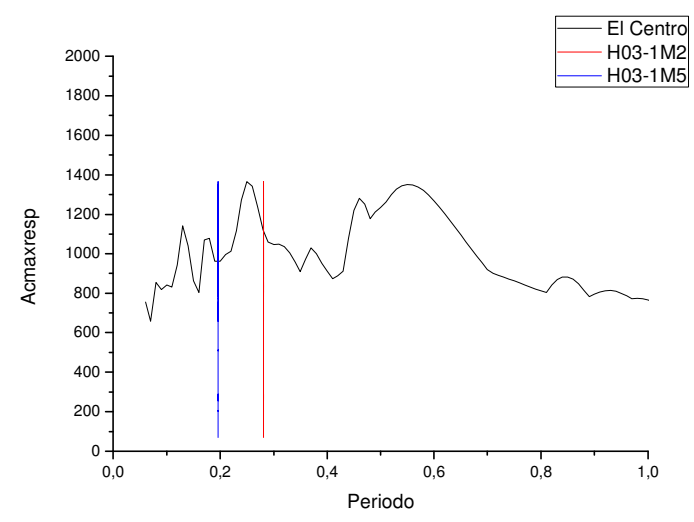

(a)

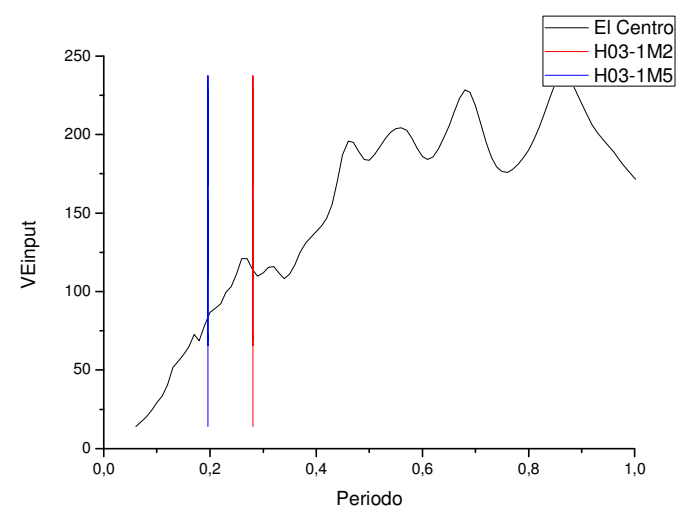

(b)

Figura 59. Espectro elástico de respuesta. El Centro. H03-1

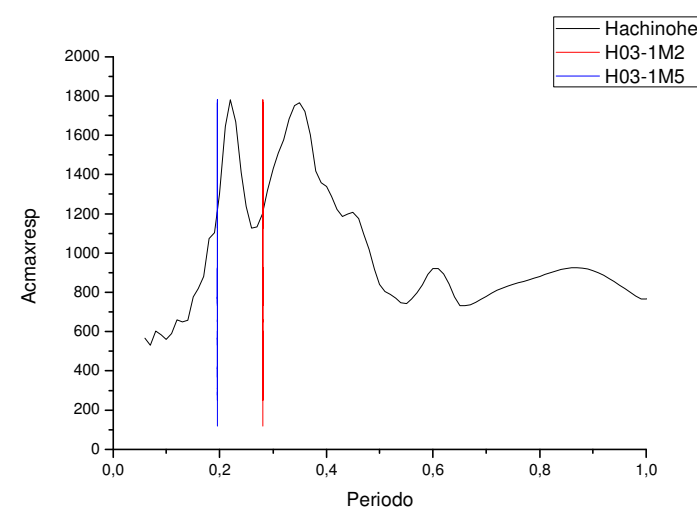

(a)

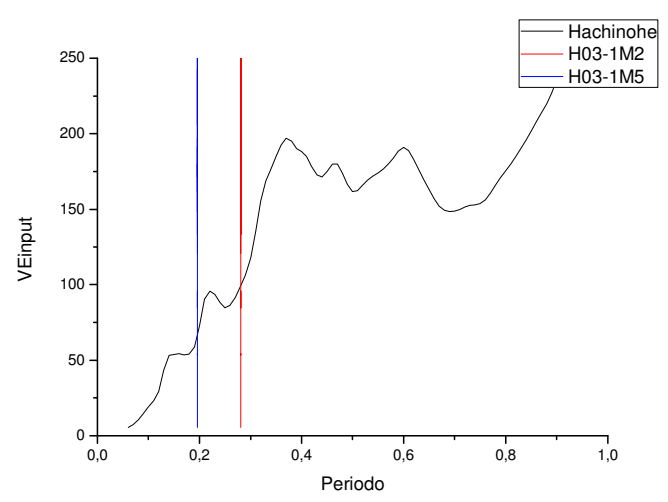

(b)

Ilustración 60. Espectro elástico de respuesta. Hachinohe. H03-1

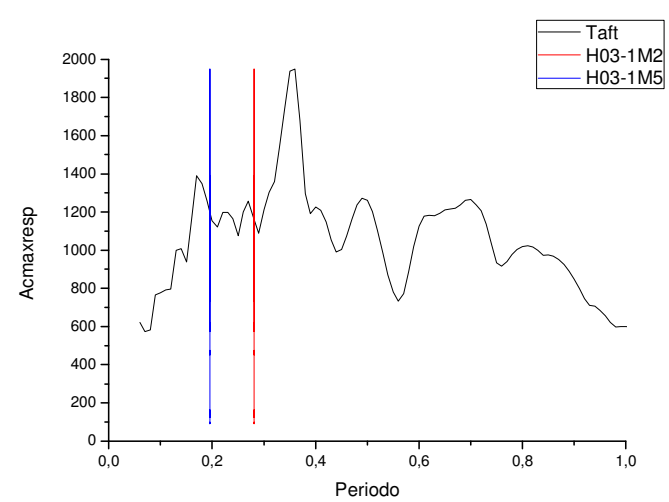

(a)

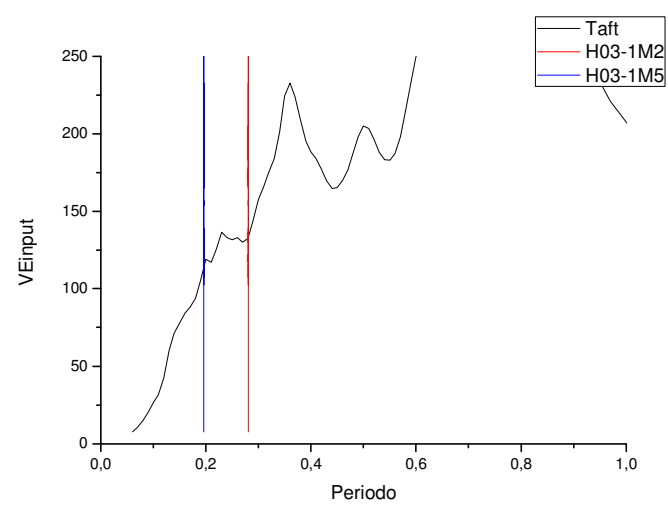

(b)

Figura 61. Espectro elástico de respuesta. Taft. H03-1 
$\mathrm{H} 03-1 \mathrm{M} 2$

El Centro 1.199

Hachinohe 45.837

TAFT 0.889

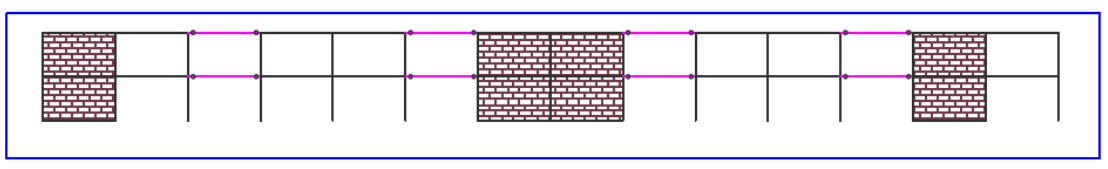

H03-1M5

El Centro 1.564

Hachinohe 0.766

TAFT 0.386

Figura 62. Modelo de prototipos para IDARC y resultado del índice de daño

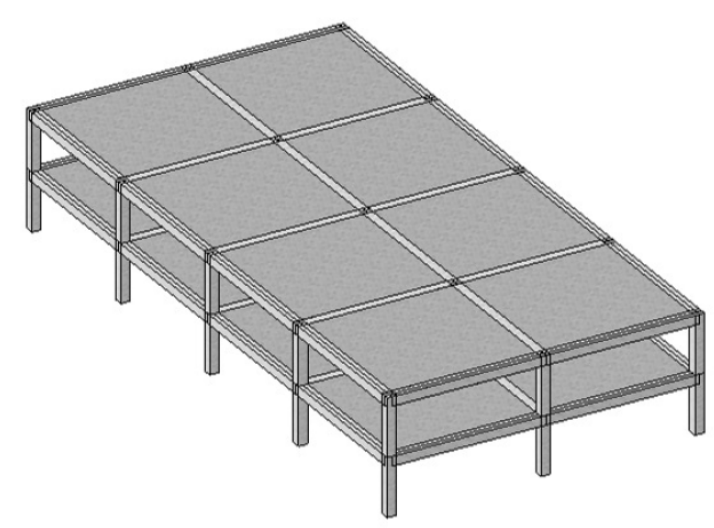

Figura 63. Prototipo H03-1

Se utilizarán para el análisis del prototipo H03-1 los resultados de las combinaciones correspondientes a:

- H03-1M2: Pese a que aparentemente no cumple la restricción de índice de daño inferior a 1para al menos dos terremotos, una fluctuación de un $20 \%$ para el terremoto de El Centro se considera apta teniendo en cuenta que para Taft el índice de daño es inferior a 1

- H03-1M5: Cumple el requisito de que el índice daño, para al menos dos terremotos, es inferior a 1. 


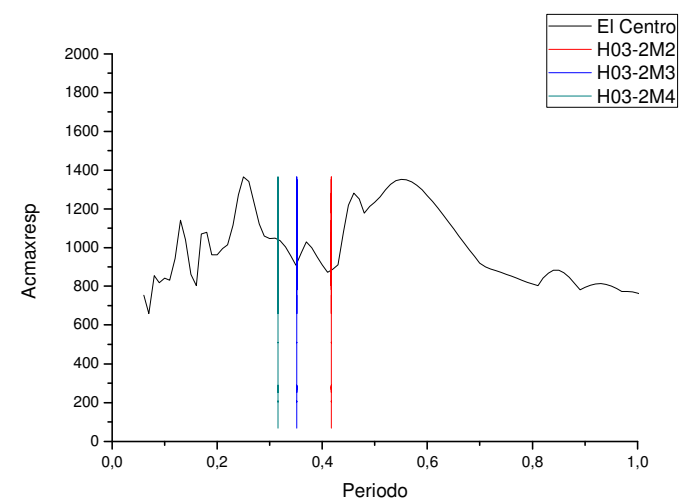

(a)

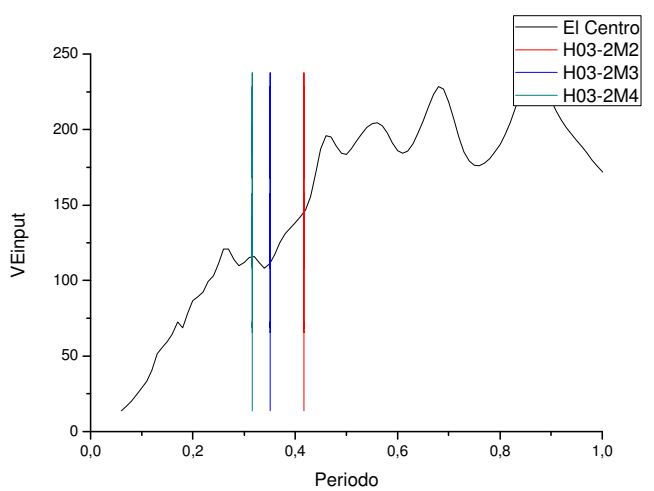

(b)

Ilustración 64. Espectro elástico de respuesta. El Centro. H03-2

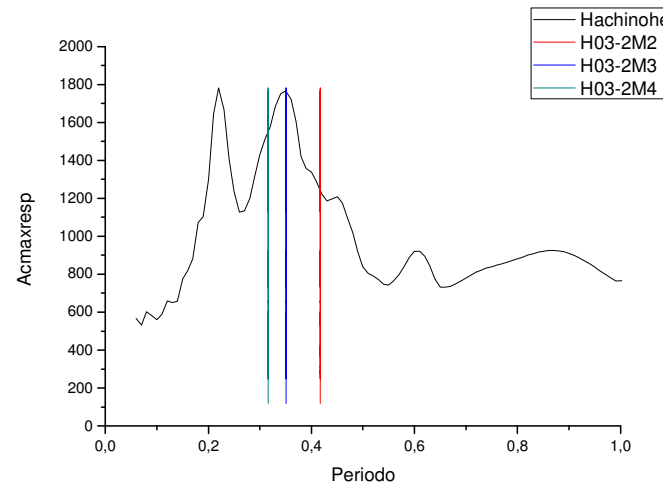

(a)

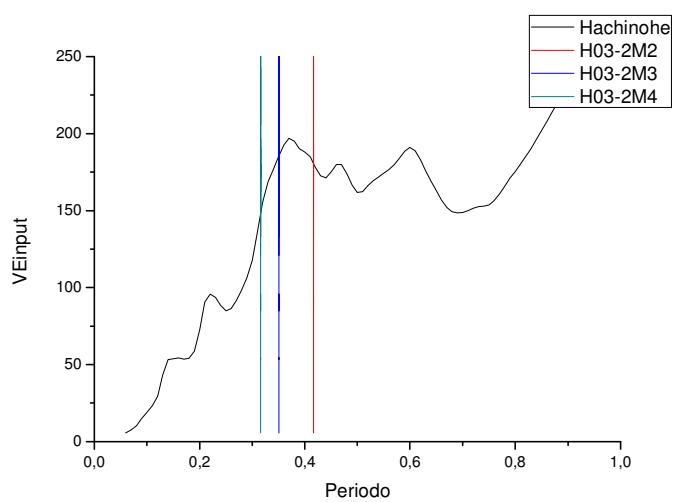

(b)

Ilustración 65. Espectro elástico de respuesta. Hachinohe. H03-2

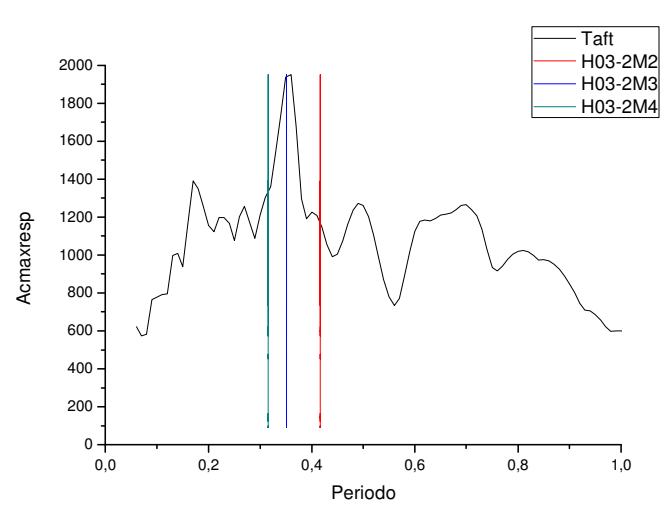

(a)

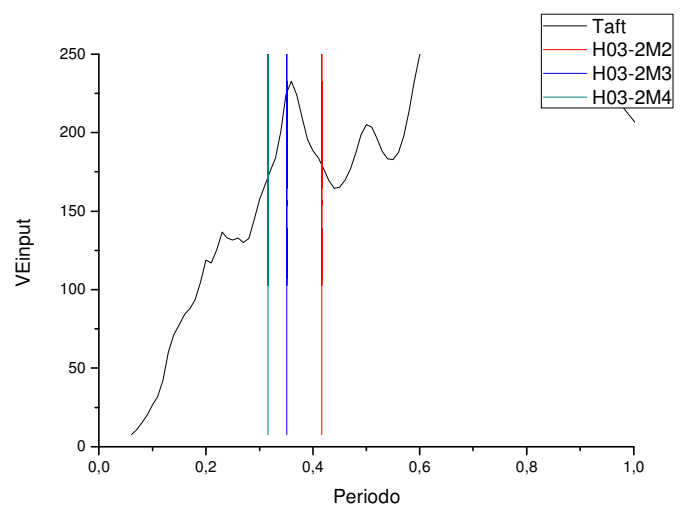

(b)

Ilustración 66. Espectro elástico de respuesta. Taft. H03-2 
H03-2M3

El Centro 0.725

Hachinohe 4.222

TAFT 0.767

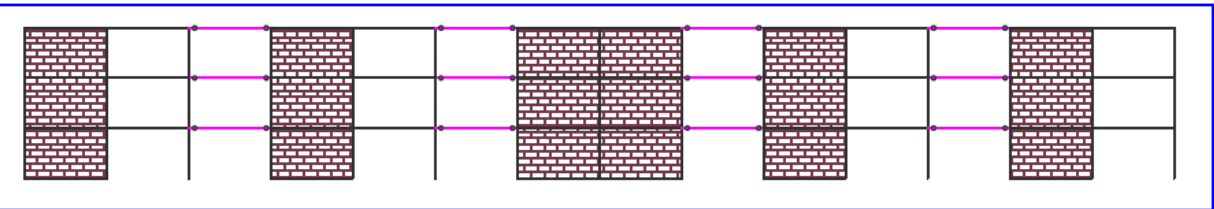

H03-2M4

El Centro 1.393

Hachinohe 0.518

TAFT 0.876

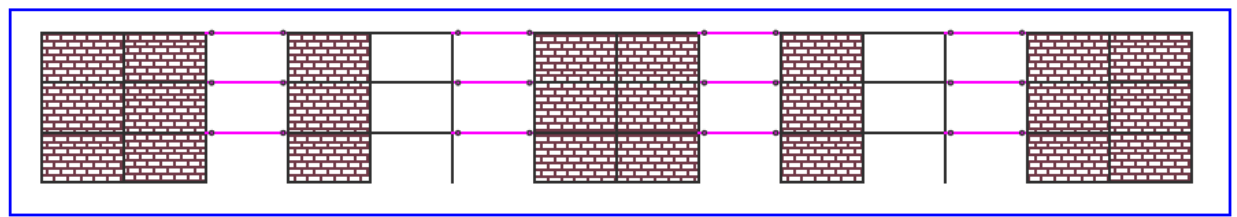

Figura 67. Modelo de prototipos para IDARC y resultado del índice de daño

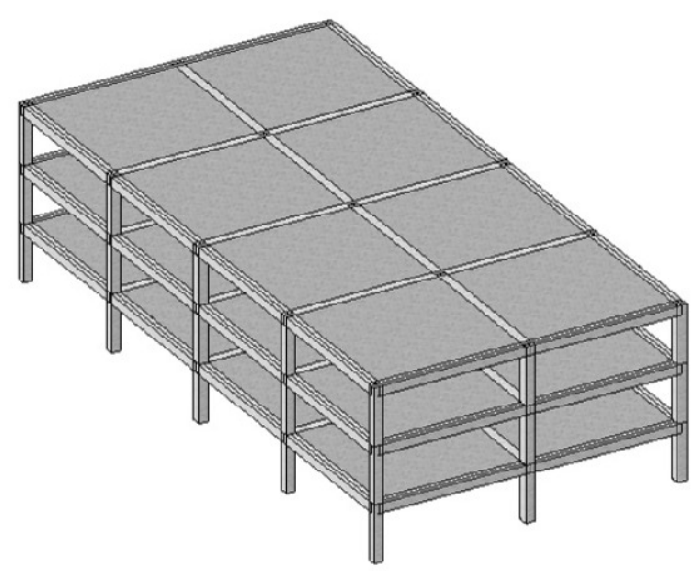

Figura 68. Prototipo H03-2

Se utilizarán para el análisis del prototipo H03-2 los resultados de las combinaciones correspondientes a:

H03-2M3: Cumple el requisito de que el índice de daño, para al menos dos terremotos, es inferior a 1.

H03-2M4: Cumple el requisito de que el índice de daño, para al menos dos terremotos, es inferior a 1. 


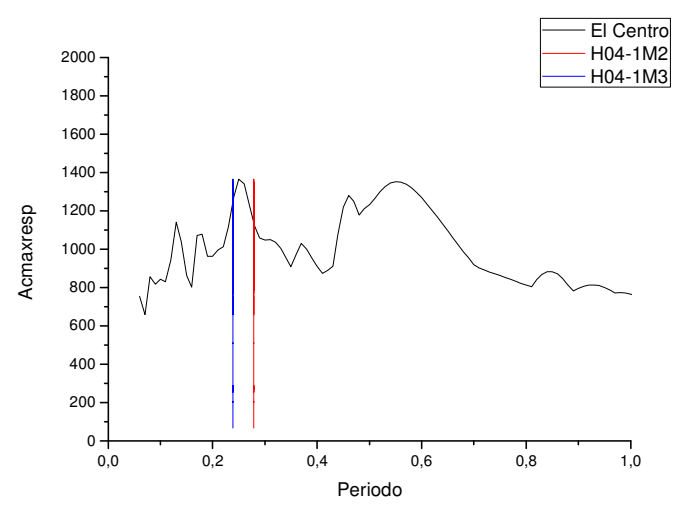

(a)

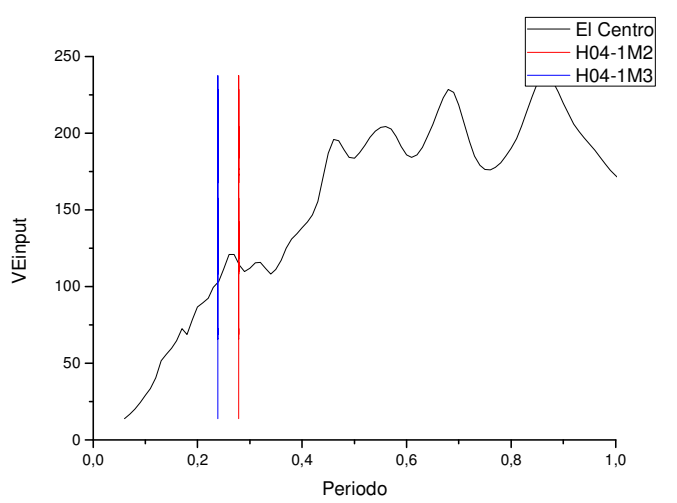

(b)

Figura 69. Espectro elástico de respuesta. El Centro. H04-1

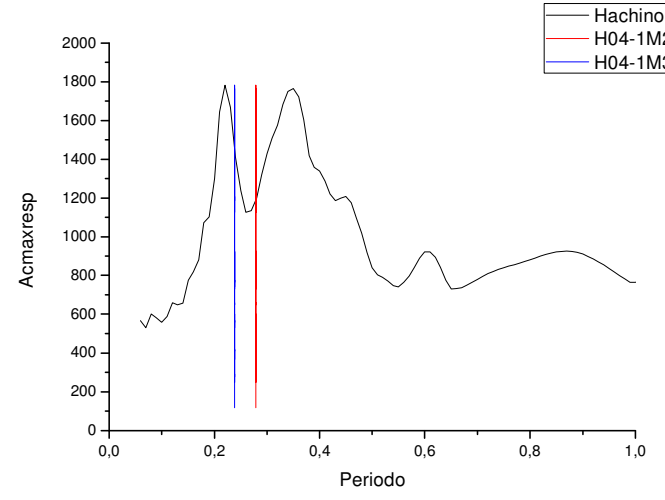

(a)

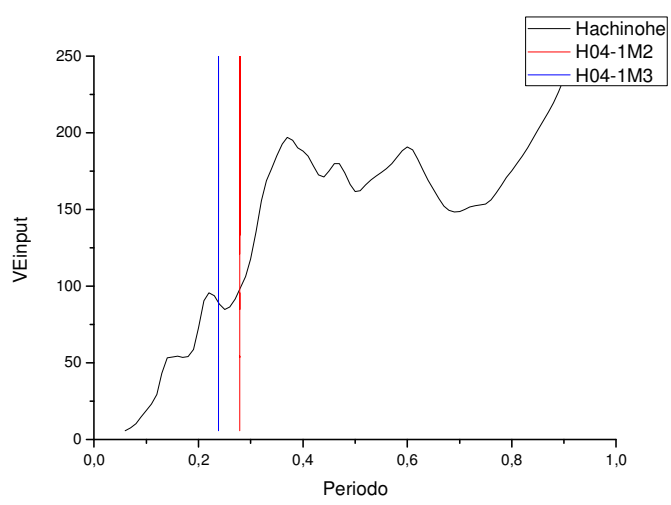

(b)

Figura 70. Espectro elástico de respuesta. Hachinohe. H04-1

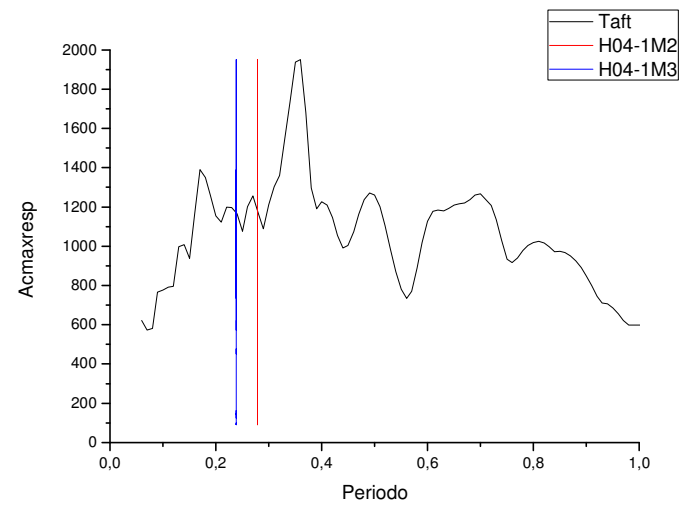

(a)

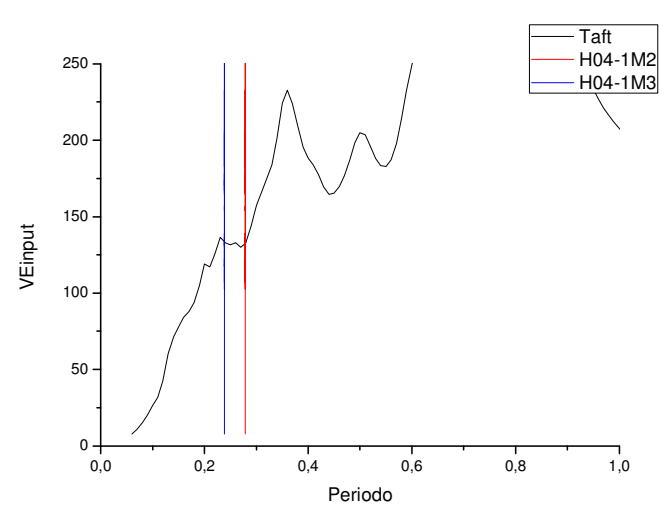

(b)

Figura 71. Espectro elástico de respuesta. Taft. H04-1 
$\mathrm{H} 04-1 \mathrm{M} 2$

El Centro 1.853

Hachinohe 1.465

TAFT 0.574

$\mathrm{H} 04-1 \mathrm{M} 3$

El Centro 0.375

Hachinohe 0.290

TAFT 1.514
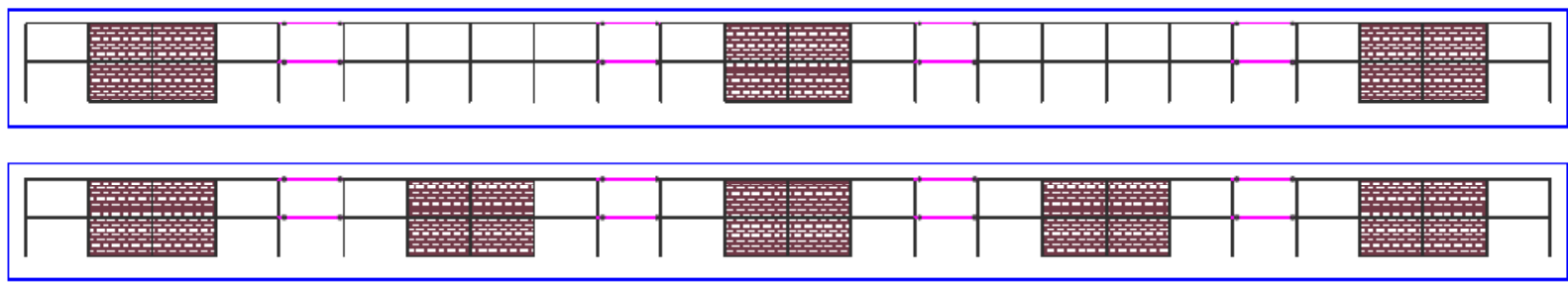

Figura 72. Modelo de prototipos para IDARC y resultado del índice de daño

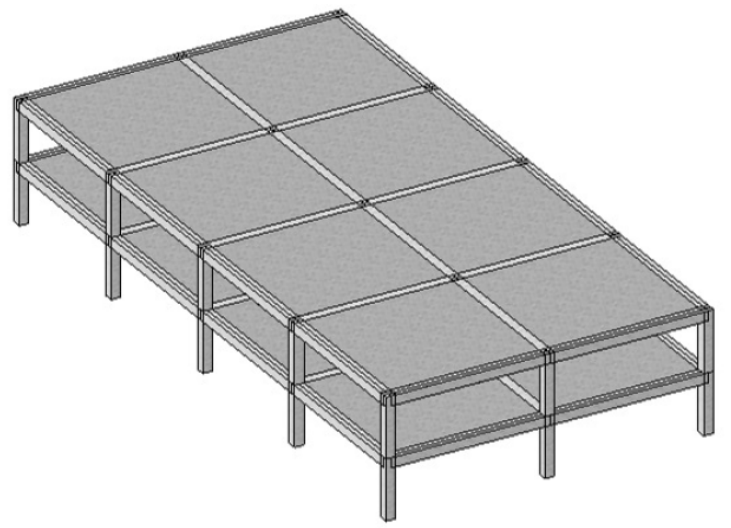

Figura 73. Prototipo H04-1

Se utilizarán para el análisis del prototipo H04-1 los resultados de las combinaciones correspondientes a:

H04-1M2: Sólo los datos correspondientes al terremoto de TAFT. Este caso es representativo porque el input de energía en este edificio para este terremoto es superior que en el caso de los terremotos de El Centro y Hachinohe, por tanto la única justificación posible para que el índice de daño sea superior en estos últimos es que el desplazamiento lateral haya sido demasiado elevado, y por ello, los resultados podrían ser engañosos

H04-1M3: Cumple el requisito de que, al menos para dos terremotos, el índice de daño de Park\&Ang es inferior a 1 


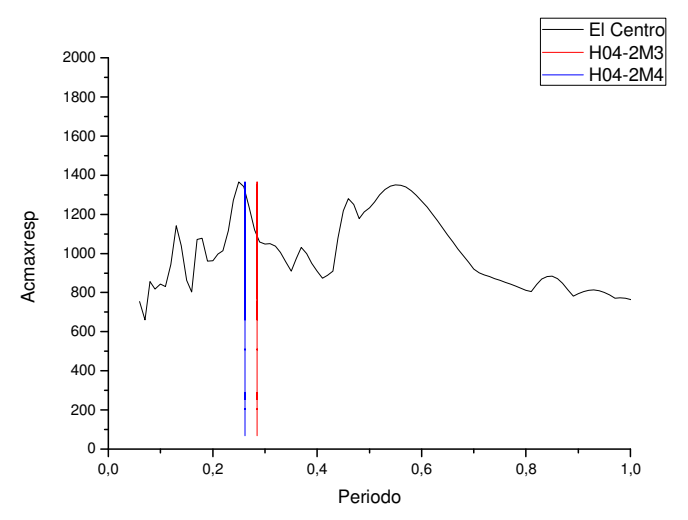

(a)

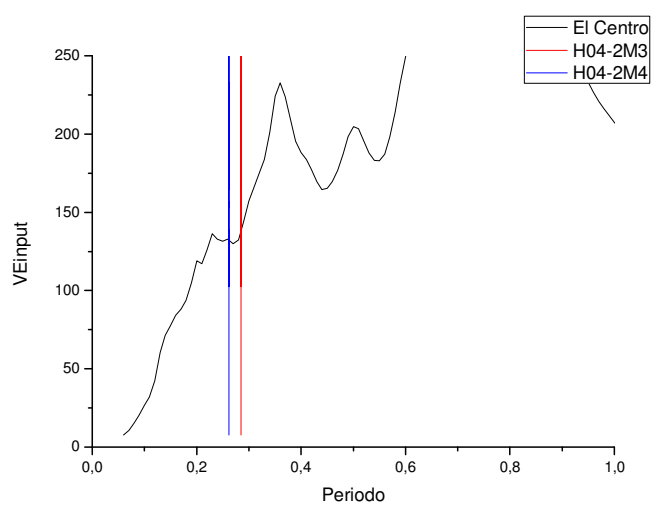

(b)

Figura 74. Espectro elástico de respuesta. El Centro. H04-2

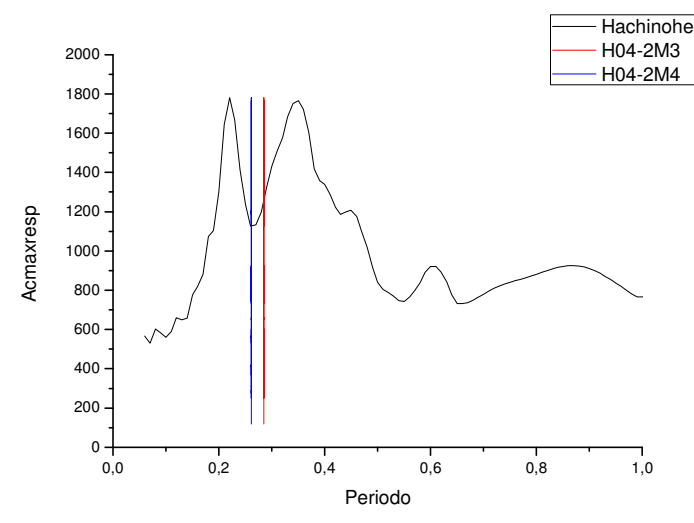

(a)

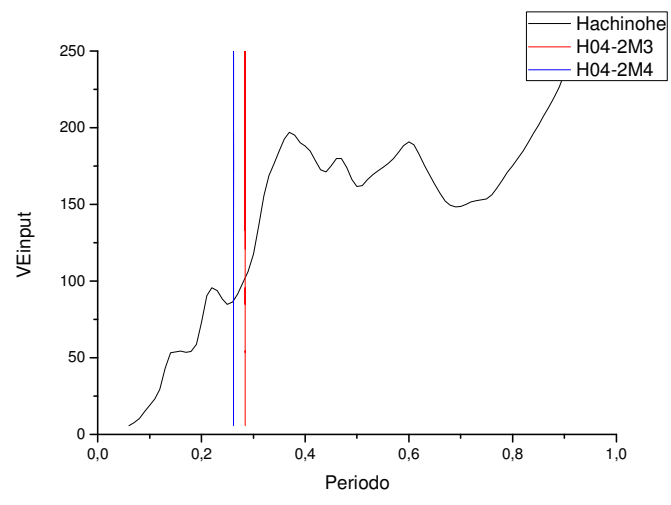

(b)

Figura 75. Espectro elástico de respuesta. Hachinohe. H04-2

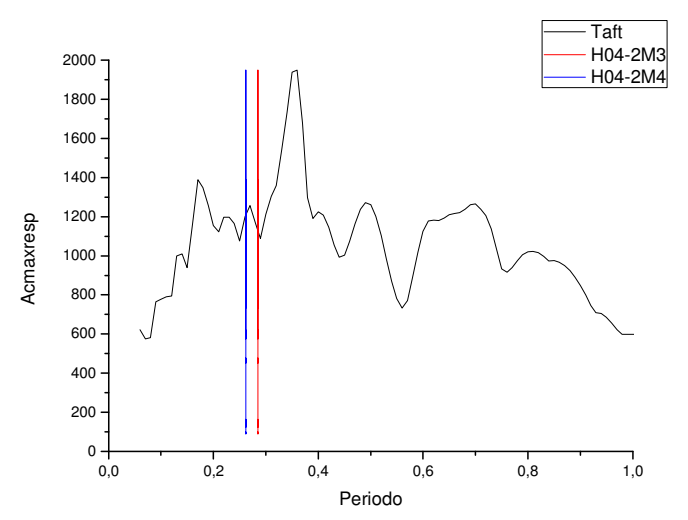

(a)

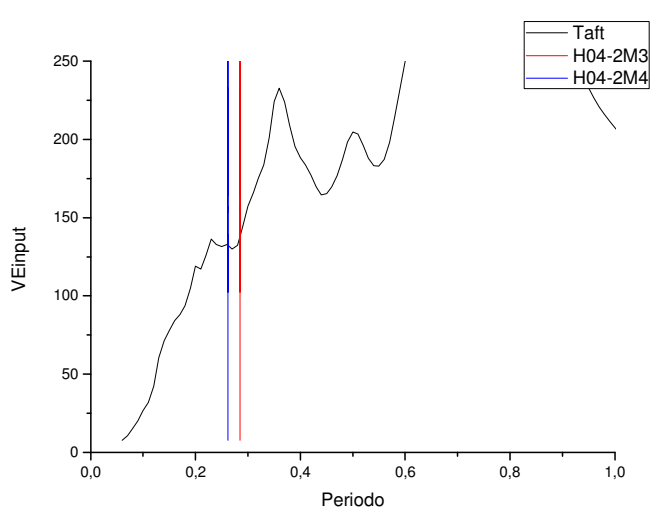

(b)

Figura 76. Espectro elástico de respuesta. Taft. H04-2 
H04-2M3

El Centro 0.593

Hachinohe 1.726

TAFT 0.594

\begin{tabular}{|c|c|c|c|c|}
\hline 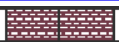 & & 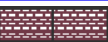 & & 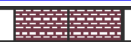 \\
\hline 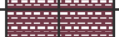 & & 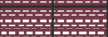 & & 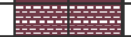 \\
\hline 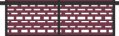 & 霜箇 & 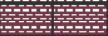 & 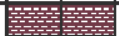 & 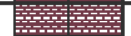 \\
\hline & 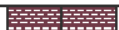 & 墿監 （ & $E^{2}$ & 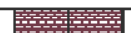 \\
\hline 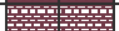 & 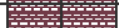 & 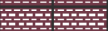 & 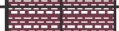 & 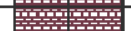 \\
\hline 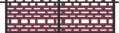 & 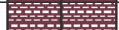 & 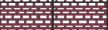 & 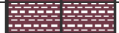 & 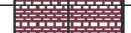 \\
\hline
\end{tabular}

$\mathrm{H} 04-2 \mathrm{M} 4$

El Centro 1.013

HAchinohe 1.179

TAFT 1.007

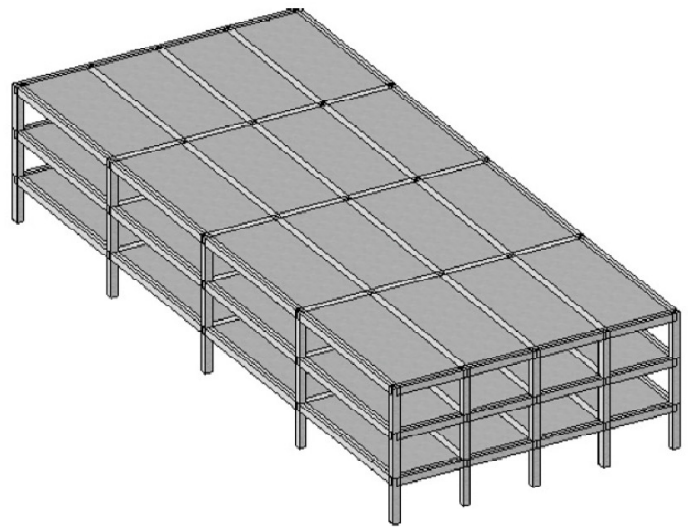

Ilustración 78. Prototipo H04-2

Se utilizarán para el análisis del prototipo H04-2 los resultados de las combinaciones correspondientes a:

- H04-2M3: Cumple el requisito de que, al menos para dos terremotos, el índice de daño de Park\&Ang es inferior a 1

- H04-2M4: Cumple el requisito de que, al menos para dos terremotos, el índice de daño de Park\&Ang es inferior a 1 


\section{CARACTERIZACIÓN DEL DAÑO EN LA ESTRUCTURA Y DEL AREA DE MUROS. RESULTADOS DE LOS CÁLCULOS DINÁMICOS DIRECTOS}

\section{CALCULOS DINÁMICOS DIRECTOS}

El cálculo dinámico directo en régimen no lineal paso a paso se ha realizado aplicando el método paso a paso de integración de las ecuaciones del movimiento con el programa IDARC. Como datos de partida al programa se le introducen:

- Identificación de cada elemento estructural y su ubicación en el espacio

- Características mecánicas de cada elemento estructural

- Tipo de ley histerética y parámetros que la gobiernan para cada elemento

- Tipo de análisis a realizar (cálculo dinámico directo), así como acelerograma y parámetros de escalado

- Tipo de amortiguamiento, que para este estudio se ha utilizado el amortiguamiento de Rayleigh

En el análisis no lineal paso a paso se va ajustado la matriz de rigidez de cada uno de los elementos estructurales y por ende la del edificio entero, siguiendo las leyes histeréticas descritas en capítulos anteriores. Los terremotos a utilizar son escalados para que se ajusten a una determinada aceleración máxima del suelo obtenida de la norma NCSE-02 y de los mapas de peligrosidad de la GSAHP.

En este capítulo se describen los detalles del cálculo dinámico directo aplicando métodos paso a paso que tiene implementado el programa IDARC. Partiendo de unas condiciones iniciales introducidas por el usuario, el cálculo dinámico directo trata de resolver el sistema de ecuaciones que se muestra a continuación:

$[M]\{\Delta \ddot{u}\}+[C]\{\Delta \dot{u}\}+\left[K_{t}\right]\{\Delta u\}=-[M]\left(\left\{L_{h}\right\} \Delta \ddot{x}_{g h}+\left\{L_{v}\right\} \Delta \ddot{x}_{g v}\right)-\left\{\Delta P_{I W}\right\}+c_{c o r r}\left\{\Delta F_{\text {err }}\right\}$

Donde:

[M] es la matriz de masas generalizada de la estructura

[C] es la matriz de amortiguamiento de la estructura

$\left[K_{\mathrm{t}}\right]$ es la matriz de rigidez secante

$\{\Delta u\},\{\Delta \dot{u}\}$ y $\{\Delta \ddot{u}\}$ son los vectores de incremento de desplazamiento, velocidad y aceleración

$\left\{L_{h}\right\}$ y $\left\{L_{v}\right\}$ son los vectores asignados a las aceleraciones del suelo horizontal y vertical respectivamente

$\Delta \ddot{x}_{g h}, \Delta \ddot{x}_{g v}$ son los incrementos de aceleración vertical y horizontal

$\left\{\Delta P_{I W}\right\}$ son las fuerzas restauradoras correspondientes a los muros de ladrillo

$c_{c o r r}$ es un coeficiente de correlación (normalmente igual a 1)

$\left\{\Delta F_{e r r}\right\}$ es el vector de fuerzas que hay que añadir para garantizar el equilibrio en la estructura 
Para obtener la solución a este sistema se recurre al algoritmo de Newmark-Beta que se base en las siguientes aproximaciones: $\{\dot{u}\}_{t+\Delta t}=\{\dot{u}\}_{t}+\Delta t\left[(1-\gamma)\{\ddot{u}\}_{t}+\gamma\{\ddot{u}\}_{t+\Delta t}\right]$

$\{u\}_{t+\Delta t}=\{u\}_{t}+\Delta t\{\ddot{u}\}_{t}+(\Delta t)^{2}\left[(0.5-\beta)\{\ddot{u}\}_{t}+\beta\{\ddot{u}\}_{t+\Delta t}\right.$

Donde $\beta$ y $\gamma$ son dos parámetros cuyo valor influye en la estabilidad del método y el amortiguamiento artificial que éste introduce en el cálculo. Para evitar este amortiguamiento artificial Newmark recomienda adoptar $\gamma=1 / 2$. En cuanto a $\beta$, si se toma $\beta=1 / 4$ la variante del método resultado se le denomina "método de la aceleración media constante" y si $\beta=1 / 6$ "método de la aceleración lineal". La primera versión es incondicionalmente estable pero menos eficiente numéricamente que la segunda.

Reordenando las ecuaciones anteriores obtenemos:

$$
\begin{aligned}
& \{\dot{u}\}_{t+\Delta t}=\left(1-\frac{\gamma}{2 \beta}\right) \Delta t\{\dot{u}\}_{t}-\frac{\gamma}{\beta}\{\dot{u}\}_{t}+\frac{\gamma}{\beta \Delta t}\{\Delta u\}_{t+\Delta t} \\
& \{\Delta \ddot{u}\}_{t+\Delta t}=\frac{1}{\gamma \Delta t}\{\dot{u}\}_{t+\Delta t}-\frac{1}{\gamma}\{\ddot{u}\}_{t}
\end{aligned}
$$

Sustituyendo estas expresiones en la ecuación de equilibrio se obtiene:

$$
\left[K_{D}\right]\{\Delta u\}_{t+\Delta t}=\left\{\Delta F_{D}\right\}
$$

Donde $\left[K_{D}\right]$ y $\left\{\Delta F_{D}\right\}$ son la rigidez dinámica equivalente y el vector de cargas

$$
\begin{gathered}
{\left[K_{D}\right]=\frac{1}{\beta(\Delta t)^{2}}[M]+\frac{\gamma}{\beta \Delta t}[C]+\left[K_{t}\right]} \\
\left\{\Delta F_{D}\right\}=-[M]\left(\left\{L_{h}\right\} \Delta \ddot{x}_{g h}+\left\{L_{v}\right\} \Delta \ddot{x}_{g v}\right)-\left\{\Delta P_{I W}\right\}+c_{c o r r}\left\{\Delta F_{e r r}\right\} \\
+\left(\frac{1}{2 \beta}[M]+\left(\frac{\gamma}{2 \beta}-1\right) \Delta t[C]\right)\{\ddot{u}\}_{t}+\left(\frac{1}{\beta \Delta t}[M]+\frac{\gamma}{\beta}[C]\right)\{\dot{u}\}_{t}
\end{gathered}
$$

$\left\{\Delta F_{D}\right\}$ es el vector de fuerzas que se aplicará en el paso siguiente.

Al final del paso $t+\Delta t$ la diferencia entre la fuerza restauradora para cada elemento calculada usando el modelo histerético $(\{R\})$, y la fuerza restauradora considerando que no hubiese cambios en la rigidez en ese paso concreto del análisis $\left(\left\{R^{\prime}\right\}\right)$ nos permite calcular la fuerza de desequilibrio:

$$
\left\{\Delta F_{\text {err }}\right\}=(\{R\})-\left(\left\{R^{\prime}\right\}\right)
$$

Esta fuerza correctiva se aplica al siguiente paso del análisis. Además

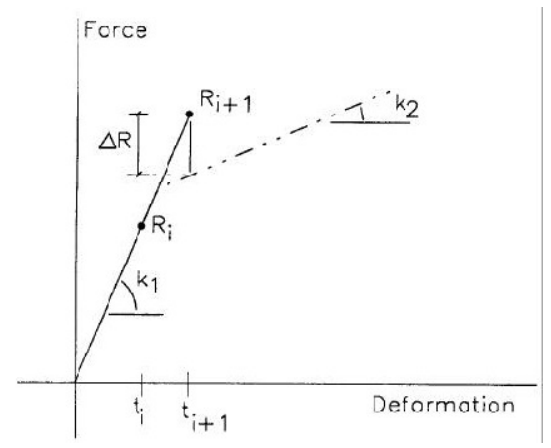
permite determinar si ha habido cambios en la matriz de rigidez de cada elemento. En caso de que esto haya ocurrido para al menos un elemento, se modifica la matriz de rigidez global de la estructura.

Para obtener el incremento de desplazamientos se resuelve el sistema dado por:

$$
\left[K_{D}\right]\{\Delta u\}_{t+\Delta t}=\left\{\Delta F_{D}\right\}
$$




\section{CARACTERIZACIÓN DEL DAÑO EN LA ESTRUCTURA DE HORMIGÓN ARMADO}

El programa IDARC permite también obtener el daño que ha sufrido cada elemento, cada planta y la estructura global mediante un índice muy empleado en estructuras de hormigón armado que es el índice de daño de Park y Ang. El índice de daño de Park y Ang para un elemento estructural viene dado por:

$$
D I_{P \& A}=\frac{\delta_{m}}{\delta_{u}}+\frac{\beta}{\delta_{u} P_{y}} \int d E_{h}
$$

Donde:

$\delta_{m}$ es la deformación máxima experimentada

$\delta_{u}$ es la deformación última del elemento

$P_{y}$ es el esfuerzo de rotura para el elemento

$\int d E_{h}$ es la energía histerética absorbida por el elemento durante la respuesta.

$\beta$ es un parámetro constante del modelo, para el que se sugiere 0.1 (Park et al. 1987)

Este índice de daño está por tanto constituido por dos sumandos, el primero depende del desplazamiento máximo experimentado por el elemento estructural o por la estructura en rango inelástico, mientras que el segundo sumando tiene en cuenta la energía de deformación plástica acumulada.

El índice de daño de Park y Ang se calcula para cada elemento por separado (columnas y vigas), para cada planta y para el global de la estructura.

El daño de un elemento se define como sigue:

$D I=\frac{\theta_{m}-\theta_{r}}{\theta_{u}-\theta_{r}}+\frac{\beta}{M_{y} \theta_{u}} E_{h}$

Donde $\theta_{m}$ es el giro máximo de la sección en el ciclo de carga correspondiente

$\theta_{u}$ es la capacidad última de rotación de la sección

$\theta_{r}$ es el giro recuperable tras la descarga

$M_{y}$ es el momento de rotura

$E_{h}$ es la energía de deformación plástica disipada por la sección

IDARC calcula el índice de daño en las dos secciones extremas de la barra y adopta como índice de daño de la misma el de mayor valor. El índice de daño de una planta o de toda la estructura se define ponderando el índice de daño $I D_{i}$ de cada planta o de toda la estructura por unos factores de ponderación $\lambda$ que se definen como sigue:

$$
D I_{\text {story }}=\sum\left(\lambda_{i}\right)_{\text {component }}\left(D I_{i}\right)_{\text {component }} ; \quad\left(\lambda_{i}\right)_{\text {component }}=\left(\frac{E_{i}}{\sum E_{i}}\right)_{\text {component }}
$$




$$
D I_{\text {overall }}=\sum\left(\lambda_{i}\right)_{\text {story }}\left(D I_{i}\right)_{\text {storyt }} ; \quad\left(\lambda_{i}\right)_{\text {story }}=\left(\frac{E_{i}}{\sum E_{i}}\right)_{\text {story }}
$$

Donde $E_{i}$ es la energía total absorbida por el componente o la planta i.

Los valores del índice de daño de Park y Ang han sido calibrados en trabajos anteriores con el daño observado en estructuras de hormigón armado tal como se indica en la Tabla 8:

Tabla 9

\begin{tabular}{|c|c|c|c|}
\hline Grado de daño & Apariencia física & Índice de daño & Estado del edificio \\
\hline Colapso & $\begin{array}{c}\text { Parcial o total } \\
\text { colapso del edificio }\end{array}$ & $>1$ & Pérdida del edificio \\
\hline Severo & $\begin{array}{c}\text { Grandes roturas en } \\
\text { el hormigón, } \\
\text { problemas en los } \\
\text { estribos }\end{array}$ & $0.4-1.0$ & Irreparable \\
\hline Moderado & $\begin{array}{c}\text { Grietas extensas, } \\
\text { desprendimiento del } \\
\text { hormigón en } \\
\text { elementos débiles }\end{array}$ & $<0.4$ & Reparable \\
\hline Menor & $\begin{array}{l}\text { Pequeñas grietas, } \\
\text { molida parcial del } \\
\text { hormigón en } \\
\text { columnas }\end{array}$ & & \\
\hline Leve & Rotura esporádica & & \\
\hline
\end{tabular}

Un índice de daño superior a 1 indica el colapso de la estructura, ocurrida bien por desplazamientos laterales excesivos, o por demandas de disipación de energía excesivas, o por una combinación de ambas.

El estudio que nos ocupa aborda estructuras porticadas, que incorporan muros de ladrillo confinados en los elementos estructurales, es decir, con en los pórticos formados por vigas y columnas de hormigón armado.

\section{CARACTERIZACIÓN DE LA CANTIDAD DE MUROS DE FÁBRICA DE LADRILLO}

Para caracterizar de una forma sencilla la cantidad de muros de fábrica de ladrillo que se han incorporado a la estructura porticada de hormigón armado en una determinada planta, en este trabajo define el ratio siguiente:

$$
R m=\frac{\text { Suma de las area de las sección transversales de los muros de la planta }}{\text { Area total de la planta }}
$$

En este trabajo el valor de este parámetro $R_{m}$ se relaciona con parámetros de respuesta de la estructura bajo diferentes terremotos. Concretamente, los parámetros de respuesta evaluados son:

- Desplazamiento entre plantas como porcentaje de la altura entre planta

- Índice de daño global de la estructura 
- Índice de daño de la planta

- Energía disipada por las rótulas plásticas de la planta

La energía disipada por las rótulas plásticas de una determinada planta se obtiene sumando el área de las curvas momento rotación de las rótulas plásticas de los pilares de esa planta y del $50 \%$ del área de las curvas momento rotación de las vigas de techo y de suelo de la planta.

En Fig. 79 se muestra una síntesis de los resultados obtenidos. En ellas han evaluado la relación entre los cuatro parámetros descritos anteriormente y el parámetro $R_{m}$. Para ello se han representado sus valores para cada una de las plantas y para cada terremoto. En las gráficas se indica con asteriscos los casos en los que los muros de dicha planta alcanzaron su capacidad última y colapsaron.

En la Fig. 79 se muestran también unas curvas que se proponen tentativamente en este trabajo como aproximación de la relación entre los parámetros de respuesta investigados y el parámetro $R_{m}$ Para la determinación de estas curvas se han utilizado los datos correspondientes al límite superior de las respuestas obtenidas y los correspondientes al $85 \%$ de las respuestas máximas obtenidas, interpolando por el método de los mínimos cuadrados, con el que obtenemos la ecuación de la curva cuya distancia a cada uno de los puntos utilizados al cuadrado es mínima. Con estas curvas se puede determinar fácilmente el área de muros por planta que habría que añadir a la estructura de hormigón armado para que, bajo el terremoto de proyecto, no se rebasase un determinado valor del parámetro de respuesta (desplazamiento máximo entre plantas, índice de daño en una planta, en todo el edificio, o energía de deformación plástica disipada). 


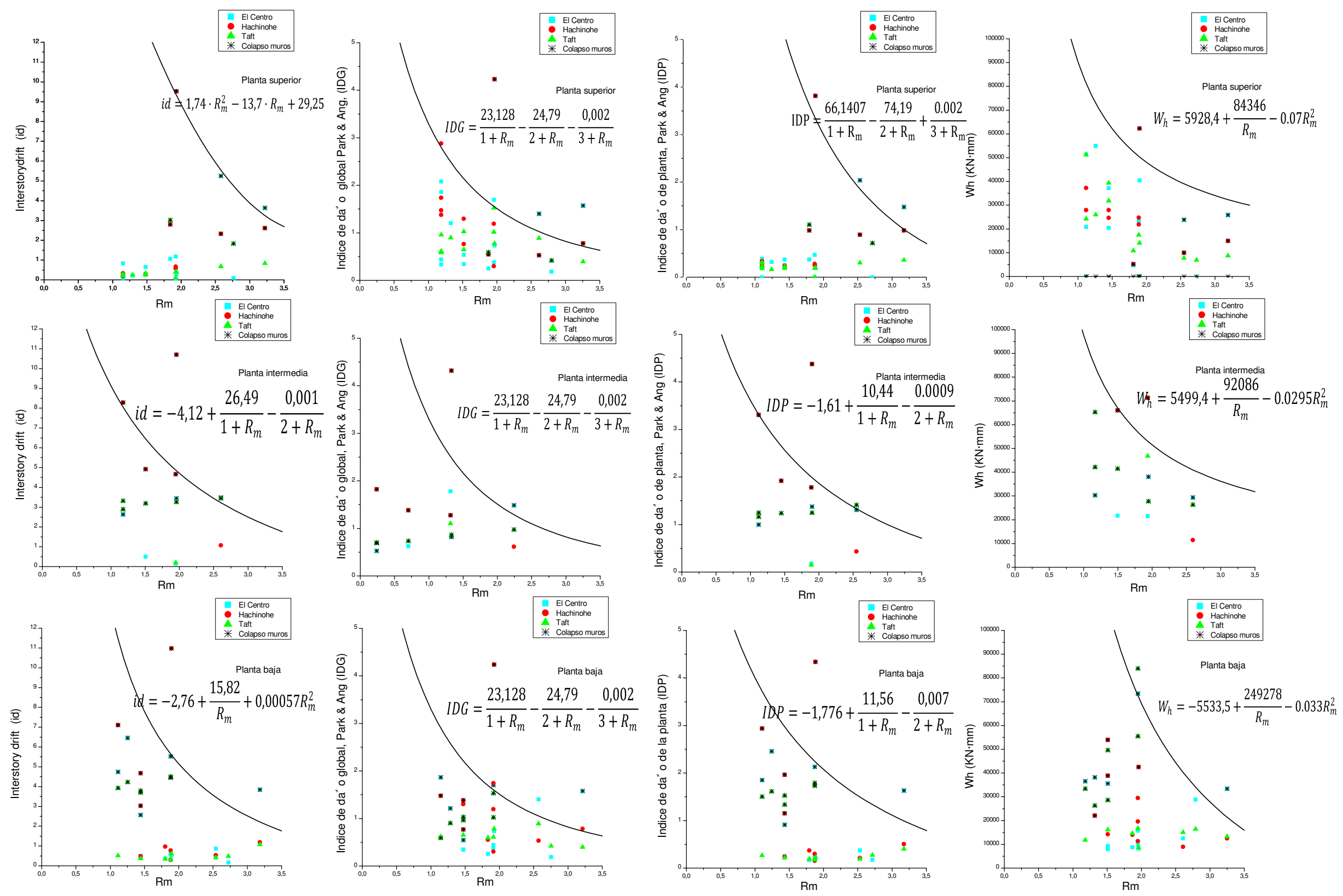




\section{CONCLUSIONES}

En este trabajo se estudia un sistema de bajo coste para el reacondicionamiento sísmico de estructuras porticadas de hormigón armado dañadas por terremotos, consistente en el relleno de vanos con muros de fábrica de ladrillo. El sistema está pensado para países en vías de desarrollo, por tanto con bajos recursos económicos, y el estudio se ha centrado en los edificios de escuelas de Haití que sufrieron daños muy importantes en el último terremoto de febrero de 2010. Estas escuelas están resueltas con estructuras cuya construcción no ha sido especialmente cuidada, y dimensionadas únicamente para cargas verticales. El trabajo conduce a una serie de recomendaciones y conclusiones de fácil interpretación sobre cómo reacondicionar sísmicamente el tipo de estructura investigadas con muros de fábrica de ladrillo.

Las ventajas del sistema de muros de fábrica da ladrillo investigado para países con bajo nivel económico, frente a otras soluciones existentes son las siguientes:

- Su bajo coste económico frente a otras soluciones viables como son el uso de disipadores de energía o de aisladores de base. Que en estos países pueden llegar a ser inviables

- Facilidad de puesta en obra sin necesidad de especialización

- Accesibilidad de la población a la materia base (bloques de ladrillo y cemento)

En nuestra opinión, este estudio es conveniente, oportuno y necesario por las siguientes razones:

- Se ha comprobado mediante simulaciones numéricas llevadas a cabo en este estudio, y a partir de la revisión de la bibliografía basada en los últimos estudios y ensayos de laboratorio, que ninguno de los prototipos representativos de los colegios de Haití soportaría otro terremoto de características similares al de febrero de 2010 en Haití sin colapsar. Este hecho es de extrema gravedad teniendo en cuenta el uso del edificio (escolar) y su elevada probabilidad de ocupación ante un nuevo sismo.

- Hasta el momento, Haití es un país que no cuenta con normativa de construcción. Tras el terremoto de Febrero de 2010 el MTPTC (Ministère des Travaux Publics Transports et Communication) ha divulgado dos documentos entre la población, "Guide Bonnes Pratiques" (Guía de buenas prácticas) y "Guide de réparations" (Guía de reparaciones). En la primera de ellas contempla la necesidad de los muros de fábrica de ladrillo en las obras de nueva construcción y la considera efectiva para edificios de máximo dos plantas, estableciendo los siguientes porcentajes de área de muro con respecto a la superficie total de la planta: 
Tabla 10

\begin{tabular}{|c|c|c|c|}
\hline \multirow{2}{*}{ Type de sol } & \multirow{2}{*}{ Description } & \multicolumn{2}{|c|}{$\begin{array}{c}\text { Surface minimale } \\
\text { au sol }\end{array}$} \\
\hline & & 1 niveau & 2 niveaux \\
\hline Dur & $\begin{array}{c}\text { Roc } \\
\text { Gravier }\end{array}$ & $1.5 \%$ & $3.0 \%$ \\
\hline Intermédiaire & $\begin{array}{c}\text { Sable compacté } \\
\text { Argile dure }\end{array}$ & $2.0 \%$ & $4.0 \%$ \\
\hline $\begin{array}{c}\text { Mou ou } \\
\text { non compacté }\end{array}$ & $\begin{array}{l}\text { Sable lâche } \\
\text { Argile molle }\end{array}$ & $2.5 \%$ & $5.0 \%$ \\
\hline
\end{tabular}

A priori los valores de la Tabla 2 no son comparables con los obtenidos en este estudio puesto que no están pensadas para reacondicionar sísmicamente estructuras existentes, sino para estructuras de nueva planta. La relación entre el área de muro necesaria según si el edificio es de 1 o 2 plantas es de 2 (es decir, a los edificios de 2 plantas se les exige el doble de área de muros que laos de 1 planta). Se establece que el porcentaje de área de muro orientado en cada dirección varía entre $1.5 \%$ y $2.5 \%$ con respecto a la superficie total de la planta. Curiosamente, en la "Guía de reparaciones", en ningún caso se hace referencia al reacondicionamiento sísmico de edificios con muros de fábrica de ladrillo. El documento se limita a indicar en qué casos es necesario reparar los muros de bloques de ladrillo y en qué casos es necesario reponerlos por unos nuevos. Se entiende, aunque la "Guía de reparaciones" no lo dice explícitamente, que cuando hay que reponer los muros se debe respetar el ratio área de muro por superficie de planta que se fija en la "Guía de buenas prácticas" para edificios de nueva planta, y que como ya se ha mencionado anteriormente, varía entre $1.5 \%$ y $2.5 \%$ en cada dirección y en cada planta.

En este contexto, las principales conclusiones y recomendaciones de este estudio que se derivan de los resultados numéricos obtenidos son los siguientes:

1.- Es recomendable que la distribución de los rellenos de muros en planta se haga manteniendo la simetría del edificio original (si la tiene), y en todo procurando que la adición de muros consiga acercar lo máximo posible el centro de masas de cada planta con el centro de torsión de la misma, para minimizar los efectos de torsión.

2.- Las estructuras objeto de estudio (pórticos de hormigón armado), por sus luces y por el hecho de haber sido proyectadas sólo para resistir cargas gravitatorias (con lo cual las secciones de las barras son relativamente pequeñas), son bastante flexibles. Sus periodos sin relleno de muros oscilan entre $0.3486 \mathrm{~s}$ y $0.7976 \mathrm{~s}$.

3. A falta de estudios de peligrosidad sísmica más específicos para Haití, en ausencia de registros de terremotos históricos medidos en la zona y de datos detallados del tipo de suelo sobre el que se cimentan los edificios de escuelas investigados, en este estudio se ha considerado a efectos de reacondicionamiento sísmico, un nivel de peligrosidad sísmica expresada en forma de aceleración máxima del suelo (PGA) de $0.525 \mathrm{~g}$. Este valor se ha basado en: (i) los mapas de peligrosidad sísmica propuestos en el "Global Seismic Hazard Assesment 
Program" que dan la PGA asociadas a un periodo de retorno de 500 años y sobre suelo duro, $y$ (ii) las fórmulas de la norma española que permiten estimar la PGA para diferentes tipos de suelo. El terremoto de provecto considerado se ha caracterizado por lo tanto con una $\underline{P G A}=0.525 \mathrm{~g}, \mathrm{y}$ a este valor se han escalado los tres acelerogramas empleados para los cálculos dinámicos directos: El Centro, Hachinohe y Taft.

4.- Teniendo en cuenta la flexibilidad lateral de las estructuras analizadas, se podrían admitir desplazamientos laterales entre plantas (id) de hasta aproximadamente $1.5 \%$ bajo la acción del terremoto de proyecto considerado. Para garantizar este desplazamiento máximo entre plantas, en este estudio se ha concluido que es necesario disponer, en la dirección del sismo, una cantidad de muros cuya sección transversal total en planta sea del 3.5\% del área total de la planta. En este estudio, a este porcentaje de área de muros en planta en relación al área total de la planta se le ha denominado $R_{m}$. Este valor de $R_{m}$ es coherente (un poco inferior) al valor del $4 \%$ que recomienda la reciente "Guía de buenas prácticas" elaborada por el gobierno de Haití en edificios de nueva planta sobre suelos de tipo intermedio.

5.- Según los cálculos numéricos realizados, este valor de $\mathrm{R}_{m}=3.5 \%$ también garantizaría que el índice de daño de Park y Ang de cada planta $(I D P)$ en el pórtico de hormigón armado se mantuviese por debajo de 1 (aproximadamente se quedaría en 0.75 ), lo cual indica daños importantes en los elementos de estructurales de hormigón armado pero sin colapsar.

6.- La distribución de daño en los pórticos de hormigón armado obtenidas de los cálculos dinámicos indica que éstos se concentran en los pilares. Es decir, el valor índice de daño de Park y Ang referido anteriormente proviene principalmente (casi exclusivamente) de las deformaciones plásticas producidas en los extremos de los pilares.

7.- Los cálculos numéricos realizados indican que este valor de $R_{m}=3.5 \%$ también garantizaría que el índice de daño de Park y Ang global para todo el edificio (IDG) se mantuviese por debajo de 1 (aproximadamente se quedaría en 0.75 ), lo cual indica daños importantes en el pórtico de hormigón armado pero sin colapsar.

8.- La energía total disipada mediante deformaciones plásticas por el pórtico de hormigón armado $W_{h}$ expresada en forma de velocidad equivalente $V_{D}=S Q R T\left(2 W_{h} / M\right)$ donde $M$ es la masa total del edificio, para $R_{m}=3.5 \%$ es aproximadamente de $250 \mathrm{~mm} / \mathrm{s}$

9.- A partir de regresiones con los datos obtenidos del los cálculos dinámicos directos, en estudio se proponen tentativamente (a falta de más datos con un número mayor de acelerogramas) unas curvas que permiten relacionar el índice $R_{m}$ con el desplazamiento máximo entre plantas (id), con el índice de daño de Park y Ang a nivel de planta (IDP) y global de todo el pórtico (IDG), y la energía de deformación histerética disipada $\left(W_{h}\right.$ ). Las fórmulas que definen estas curvas son las siguientes: 
Universidad de Granada-Trabajo Fin de Máster
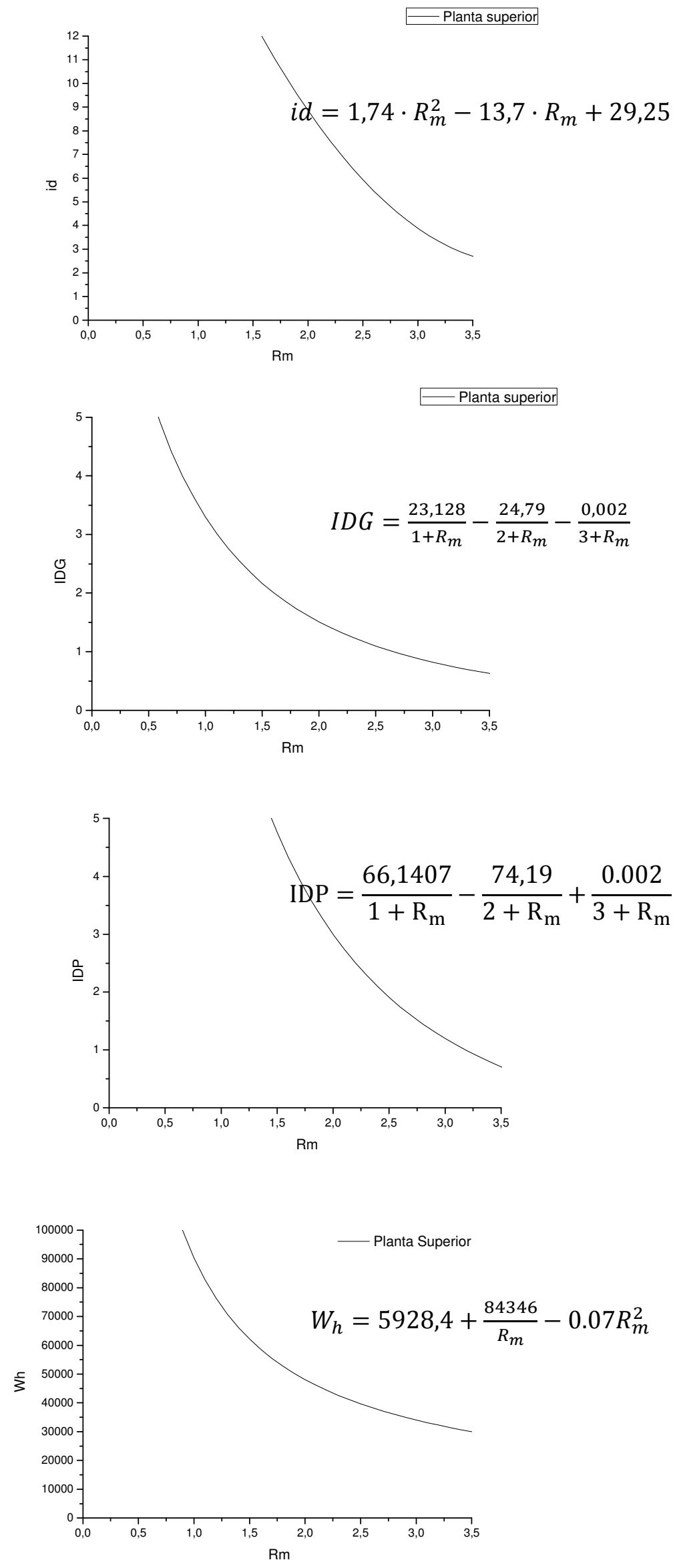

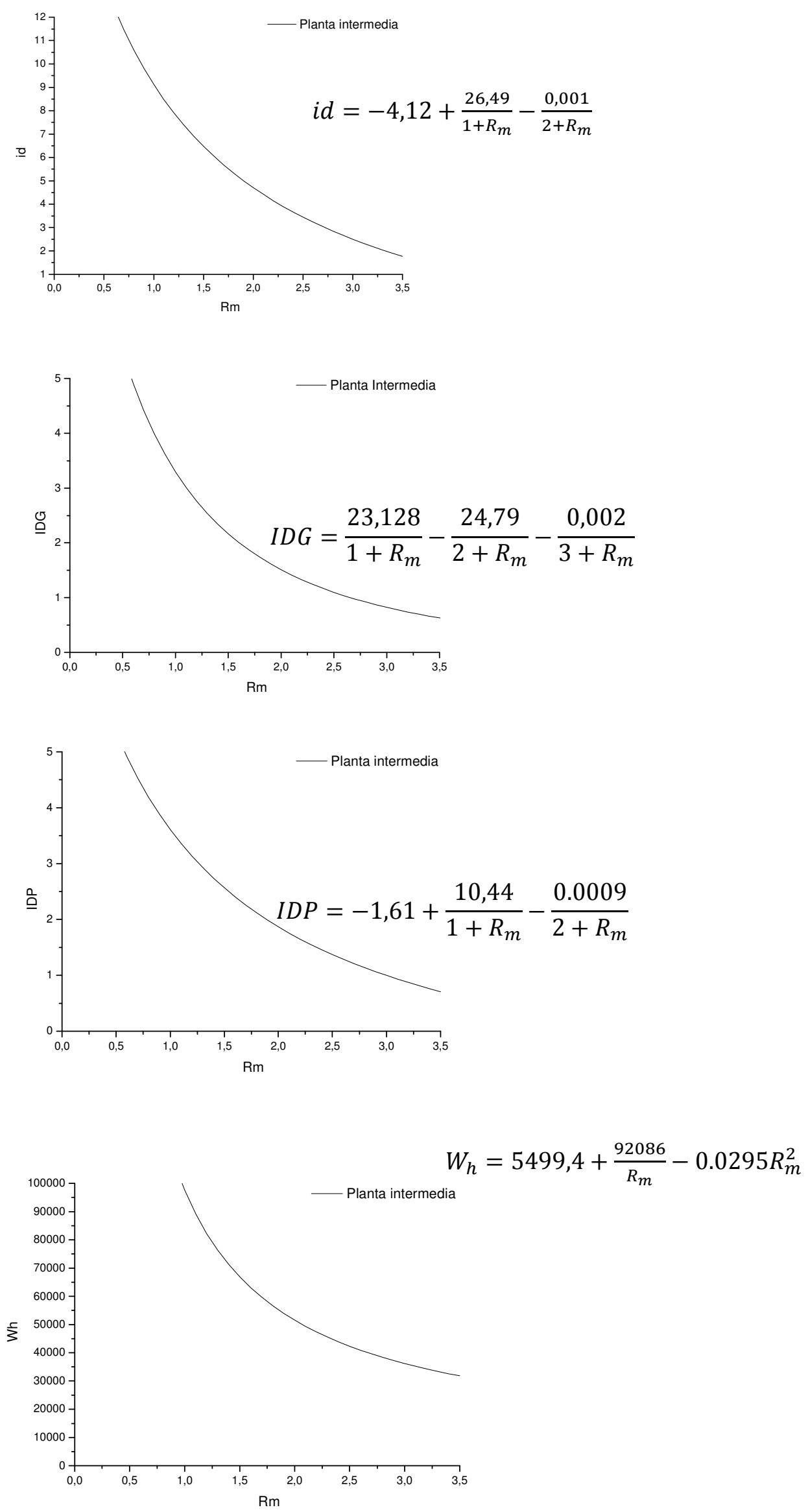

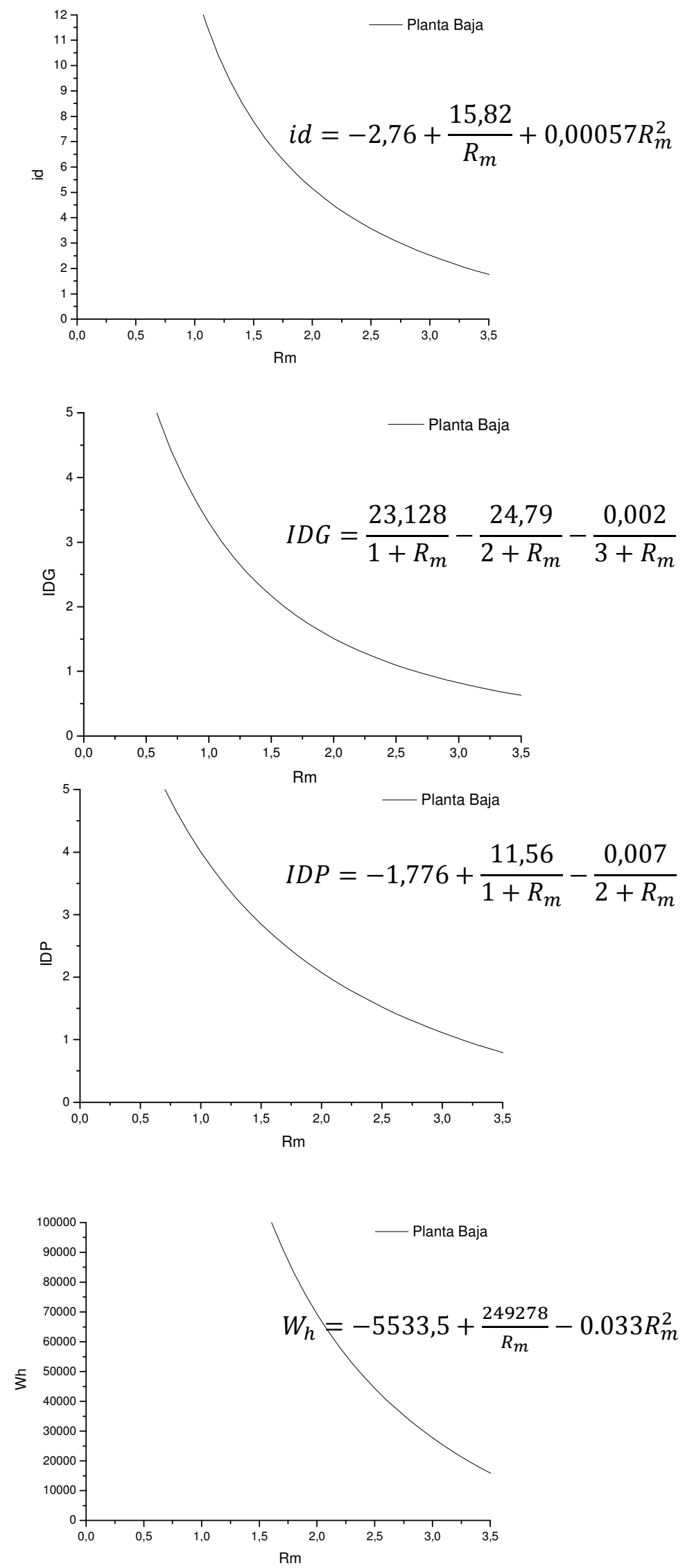


\section{LIMITACIONES DE LA INVESTIGACIÓN Y TRABAJO FUTURO}

El presente estudio investiga una solución de bajo coste para el reacondicionamiento sísmico de estructuras de hormigón armado del tipo empleado en Haití para edificios de escuelas, basado en el relleno de vanos con muros de fábrica de ladrillo. El estudio se ha basado en cálculos dinámicos directos con acelerogramas históricos. Sin embargo no se han tenido en cuenta todas las variables del problema. Entre ellas se destaca:

- Efectos de torsión en el edificio

- Posibles giros en las zapatas y consecuentemente en los arranques de los pilares

- La influencia de los muros transversales, es decir, perpendiculares a la dirección del sismo considerada.

Otra limitación del estudio es el limitado número de acelerogramas empleado (3).

En futuras investigaciones se debería contemplar diferentes leyes histeréticas para los muros (asociadas a distintos tipos de mortero, diferentes tipos de ladrillo, y distintas proporciones de juntas respecto al área total del muro), así como nuevas soluciones de encuentro entre muro y marco perimetral que forman pilares y vigas del pórtico y la influencia de la cimentación en el aumento de rigidez y resistencia del edificio.

También es muy importante validar la respuesta dinámica obtenida de las simulaciones numéricas con evidencias experimentales (ensayos con mesa sísmica).

El estudio de todos estos aspectos así como la realización de ensayos dinámicos con mesa sísmica es algo que pretende se abordar en un futuro próximo, en el marco de una Tesis Doctoral. 


\section{BIBLIOGRAFÍA}

[REF.1] Santiago Pujol, Amadeo Benavent-Climent, Mario E. Rodríguez, J. Paul Smith Pardo (2008); "Masonry infill walls: an effective alternative for seismic strengthening of low-rise reinforced concrete buildings"; The 14TH World Conference on Earthquake Engineering, October 12-17, 2008, Beijing, China

[REF. 2] G. Michael Calvi, Davide Bolognini, Andrea Penna (2004); "Seismic performance of masonry-infilled R.C. frames: Benefits of slight reinforcements"; SÍSMICA 2004, 60 Congresso Nacional de Sismología e Engenharia Sísmica

[REF. 3] Armin B. Meharabi, P. Benson Shing, Michael P.Schuller, James L. Noland (1996); "Experimental evaluation on masonry-infilled RC frames"; 228 Journal of Structural engineering, March 1996

[REF. 4] Paolo Negro, Guido Verzeletti (1996); "Effects of infills on the global behavior of R/C frames: energy considerations from pseudodynamic test"; Earthquake Engineering and Structural Dynamics, VOL 25, 753-773 (1996)

[REF. 5] Alidad Hashemi, Khalid M. Mosalam (2006); "Shake table experiment on reinforced concrete structure containing masonry infill wall"; Earthquake Engineering and Structural Dynamics, VOL 35, 1827-1852 (2006)

[REF. 8] J Song, A D. Kiureghian (2006); "Generalized Bouc-Wen model for highly asymmetric hysteresis"; Journal of Engineering Mechanics-ASCE. 1336 (6), pp. 610-618 (2006)

[REF. 7] A.M. Reinhorn, R. E. Valles (1995); "Damage evaluation in Inelastic Response of Structures: A Deterministic Approach", Report No. NCEER-95-XXXX, National Centre of Earthquake Engineering Research, State University at New York at Buffalo

[REF. 8] Thomas T. Baber, Mohammad N. Noori(1985); "Random Vibration of Degrading, Pinching Systems"; Journal on Engineering Mechanics, No 8, August 1985, pp 1010-1026

[REF. 9] Özgür Anil, Sinan Altin (2007), "An experimental study on reinforced concrete partially infilled frames"; Engineering Structures, 29 (2007) 449-460

[REF. 10] Matjaz Dolsek, Peter Fajfar(2005); "Simplified non-linear seismic analysis of infilled reinforced concrete frames", Earthquake Engineering and Structural Dynamics, VOL 34, 49-66

[REF. 11] Matjaz Dolsek, Peter Fajfar(2002);"Mathematical modeling of an infilled RC frame structure based on the results of pseudo-dynamic test"; Earthquake Engineering and Structural Dynamics, VOL 31, 1215-1230

[REF. 12] Matjaz Dolsek, Peter Fajfar (2004), "Inelastic spectra for infilled reinforced concrete frames"; Earthquake Engineering and Structural Dynamics, VOL 33, 1395-1416

[REF. 13] Hiroshi Akiyama, "Metodología del proyecto sismorresistente de edificios basada en el balance energético", Editorial Reverté, S. A.

[REF. 14] Amadeo Benavent-Climent, "Estructuras sismorresistentes"; Editorial Maia 
[REF. 15] Alain Pecker, "Dynamique des structures et des ouvrages"; Écoles des Ponts, ParisTech, Edition 2009

[REF. 16] Abolghasem Saneinejad, Brian Hobbs (1993); "Inelastic Design of infilled frames" ; Journal of Structural Engineering, 634-650

[REF. 17] Manual de instrucciones. Tricalc 6.2. Cálculo Especial de Estructuras Tridimensionales Capítulo 5. Aktec

[REF. 18] R. E. Valles, A. M. Reinhorn, S. K. Kunnath, C. Li, A, Madan (1996) ; "IDARC 2D Version 4.0:A Program for the Inelastic Damage Analysis of Buidings " National Center for Earthquake Engineering Research, State University of New York at Buffalo ; NCEER task Numbers 943103A and $943101 \mathrm{~A}$ 


\section{PLANOS DE ESTRUCTURA}



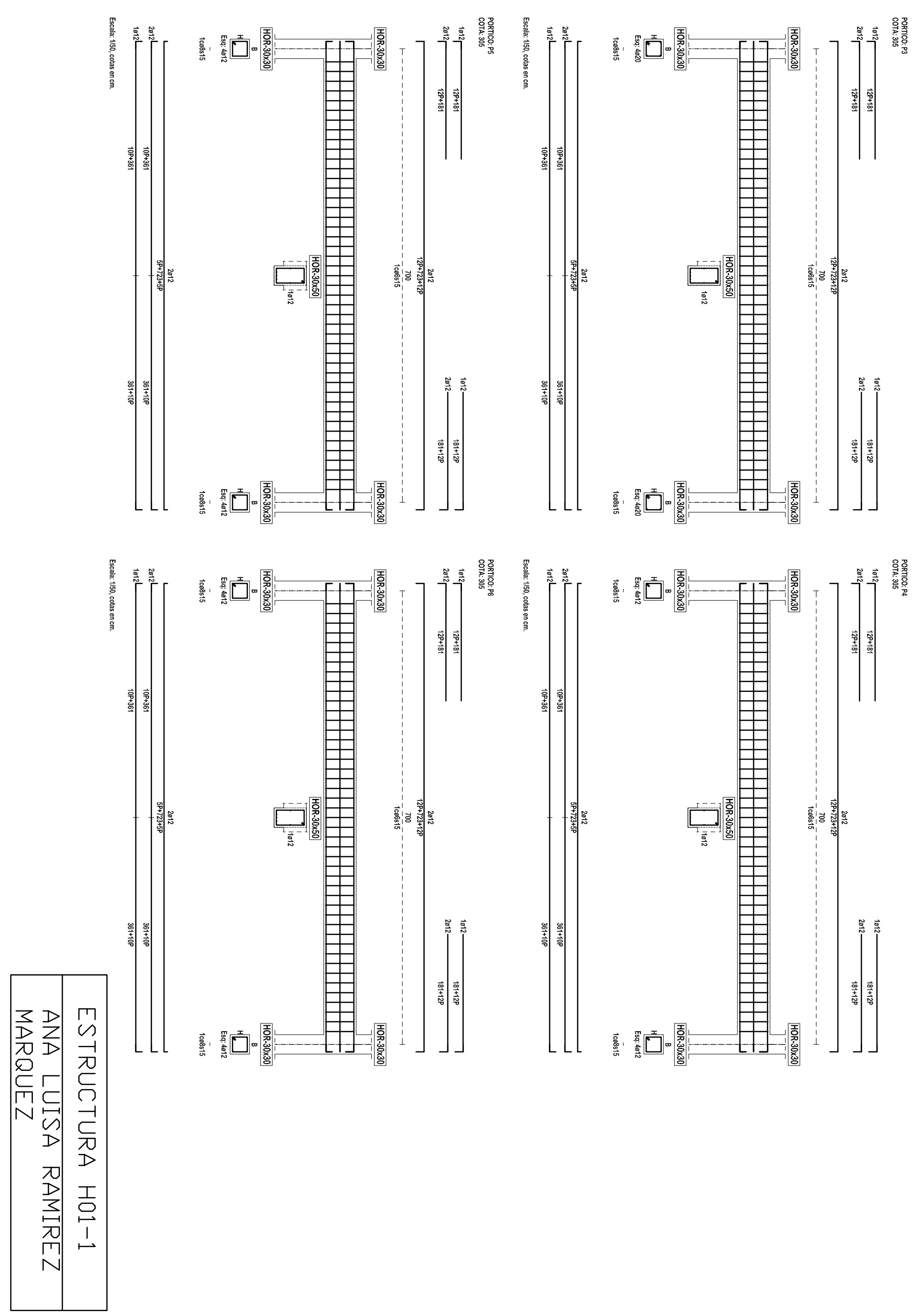

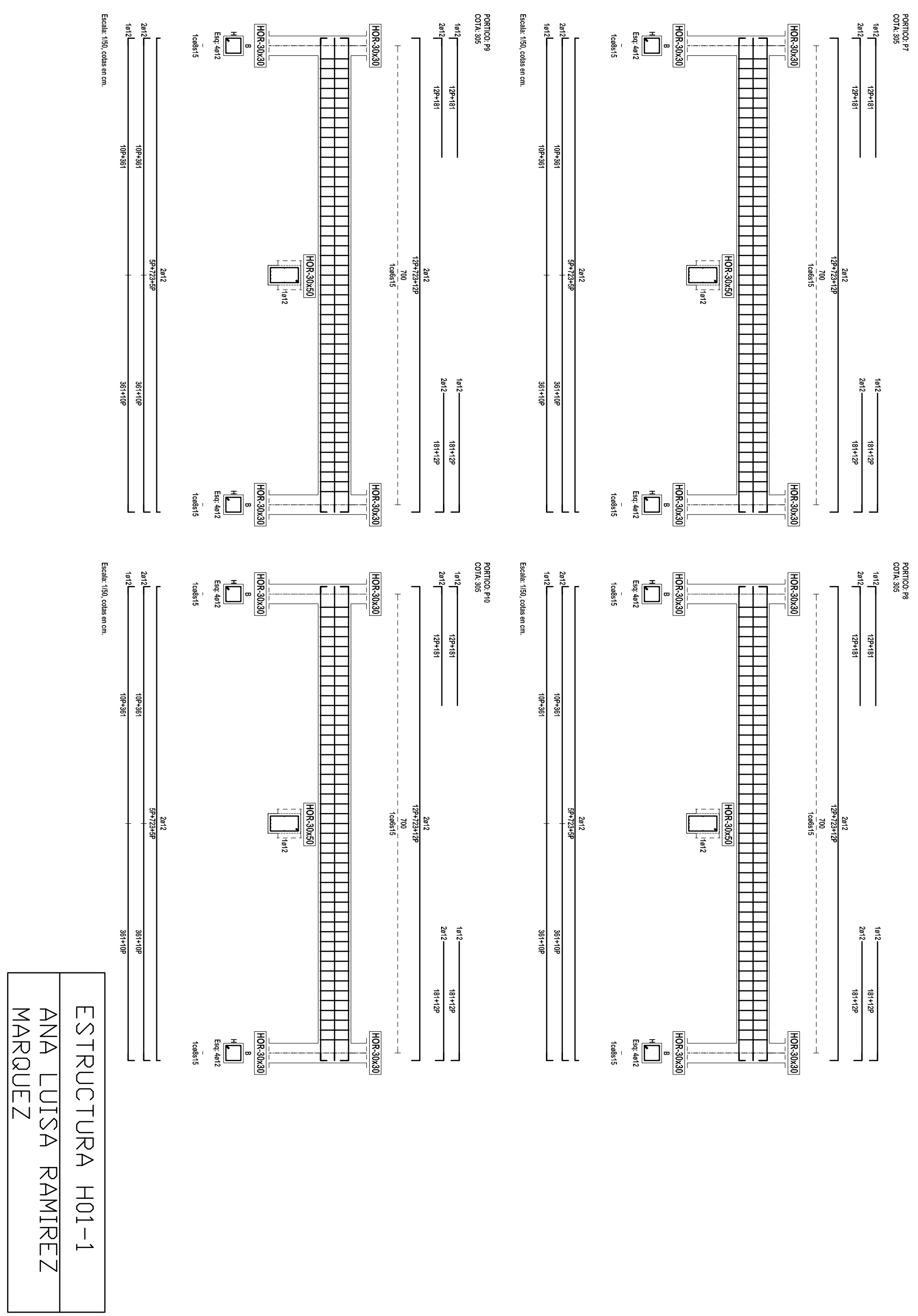


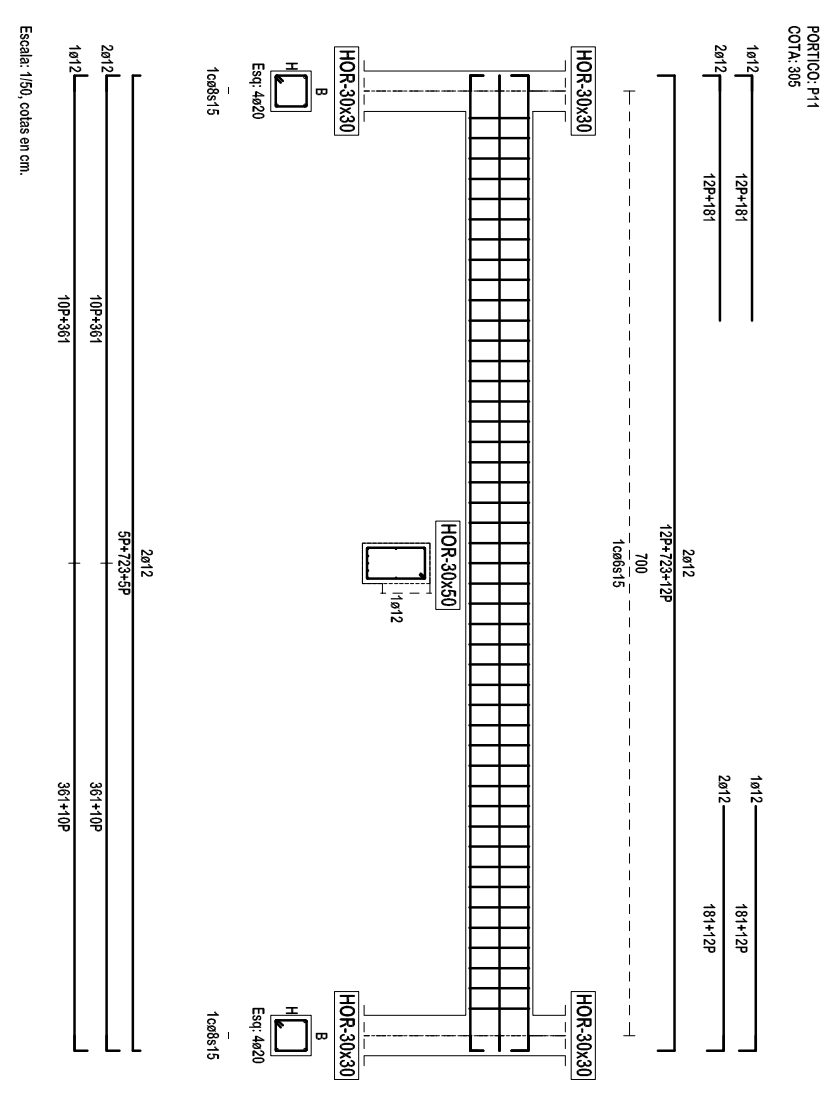

\begin{tabular}{ll|l}
3 & $D$ & $\Pi$ \\
$D$ & $Z$ & $\ddots$ \\
\hdashline & $D$ & -1
\end{tabular}

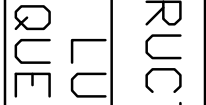
N

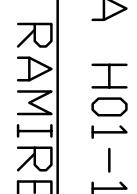
N 

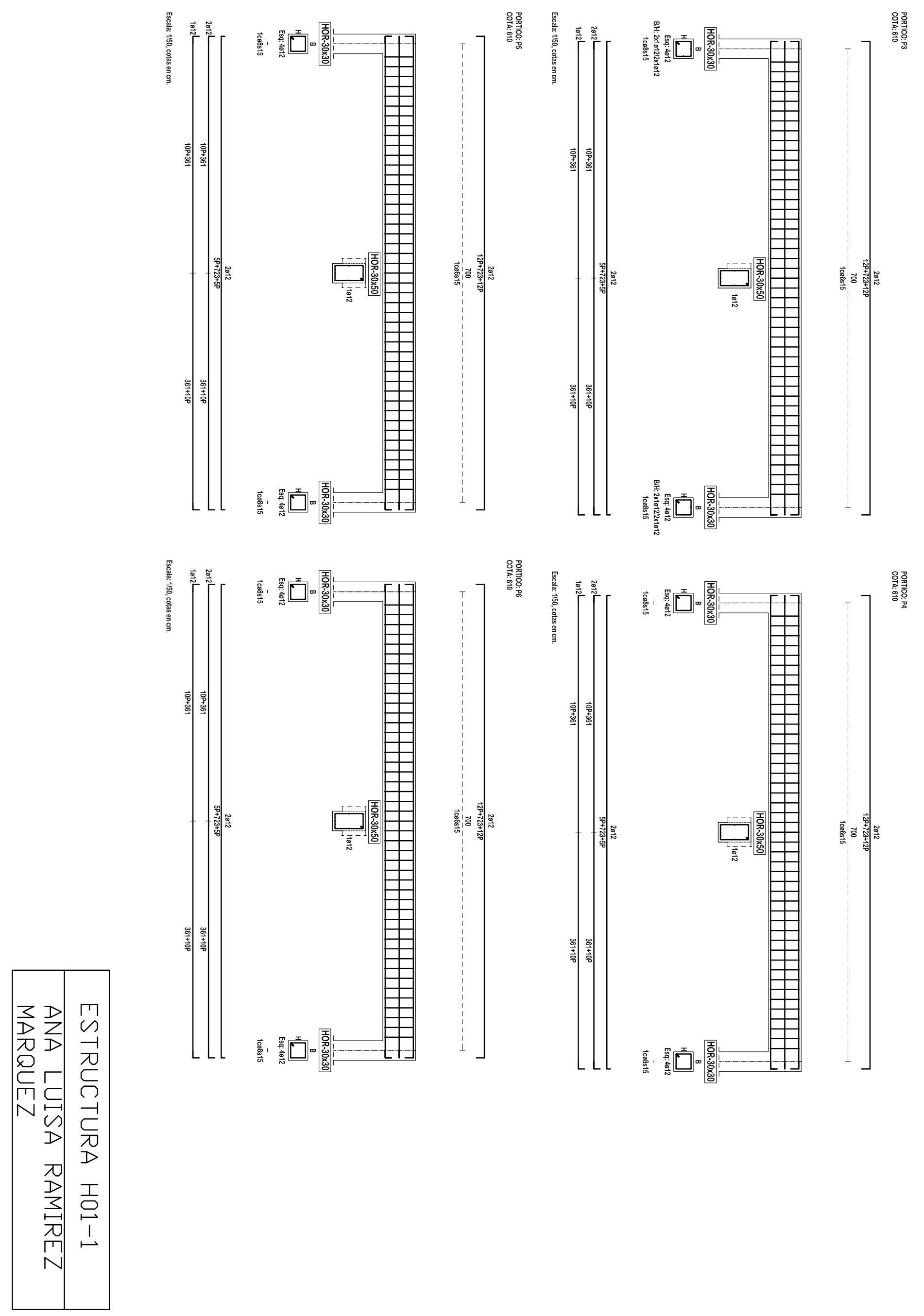

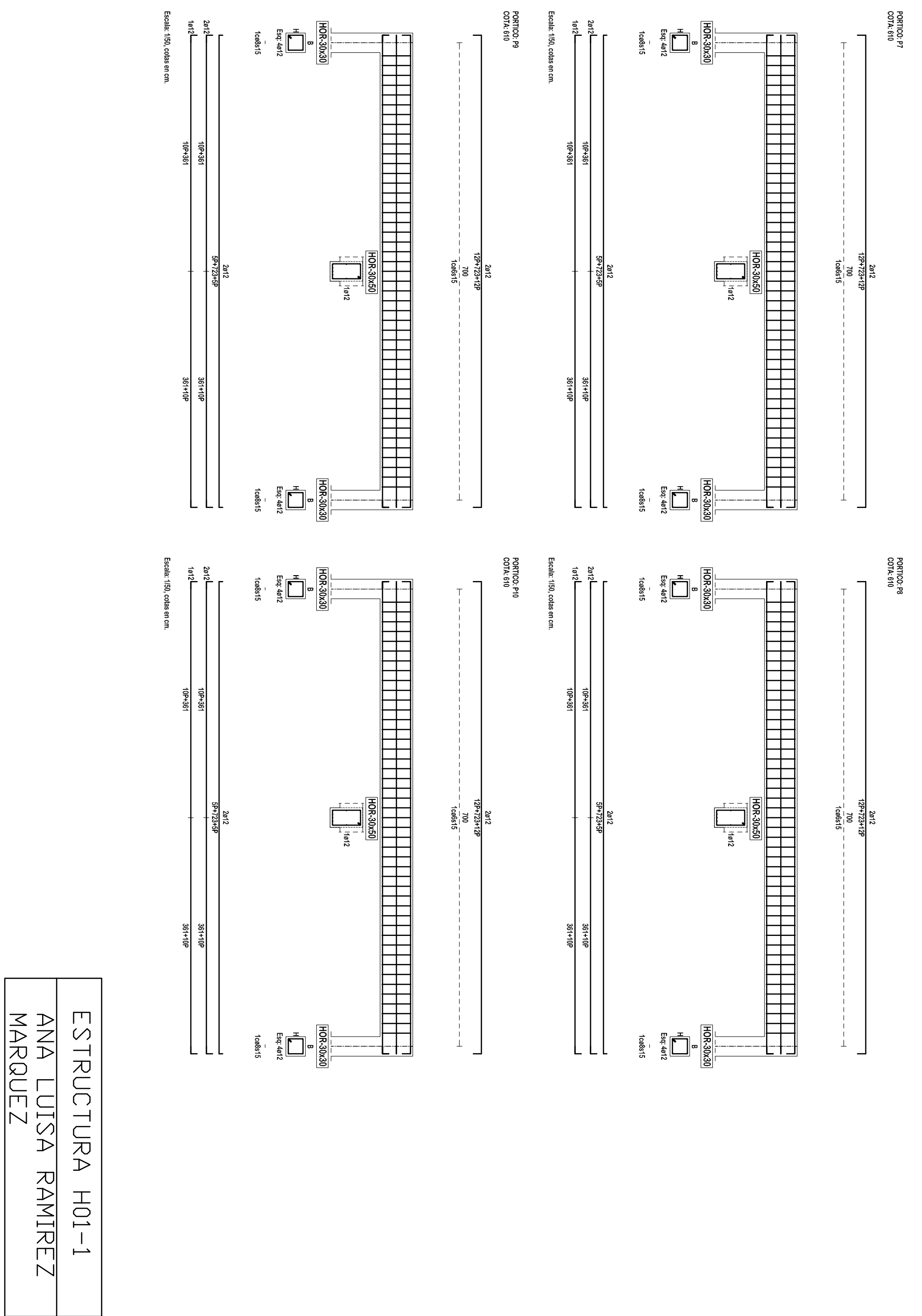


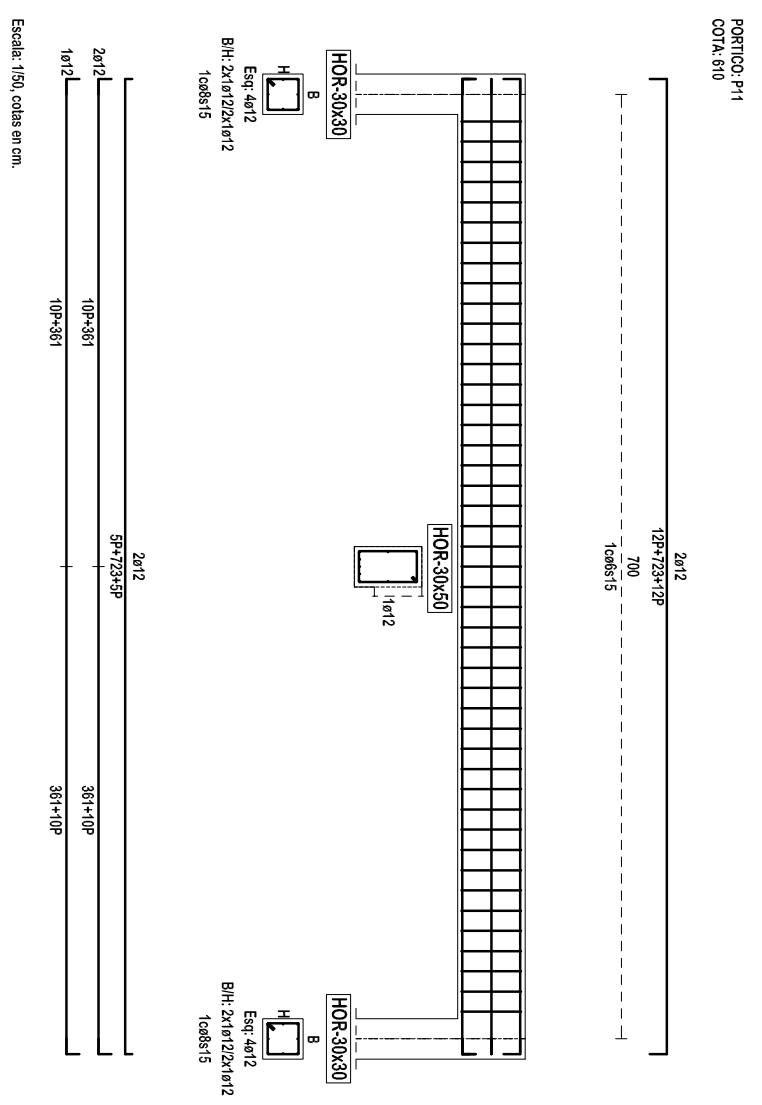

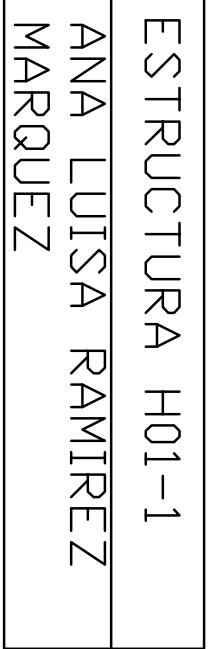



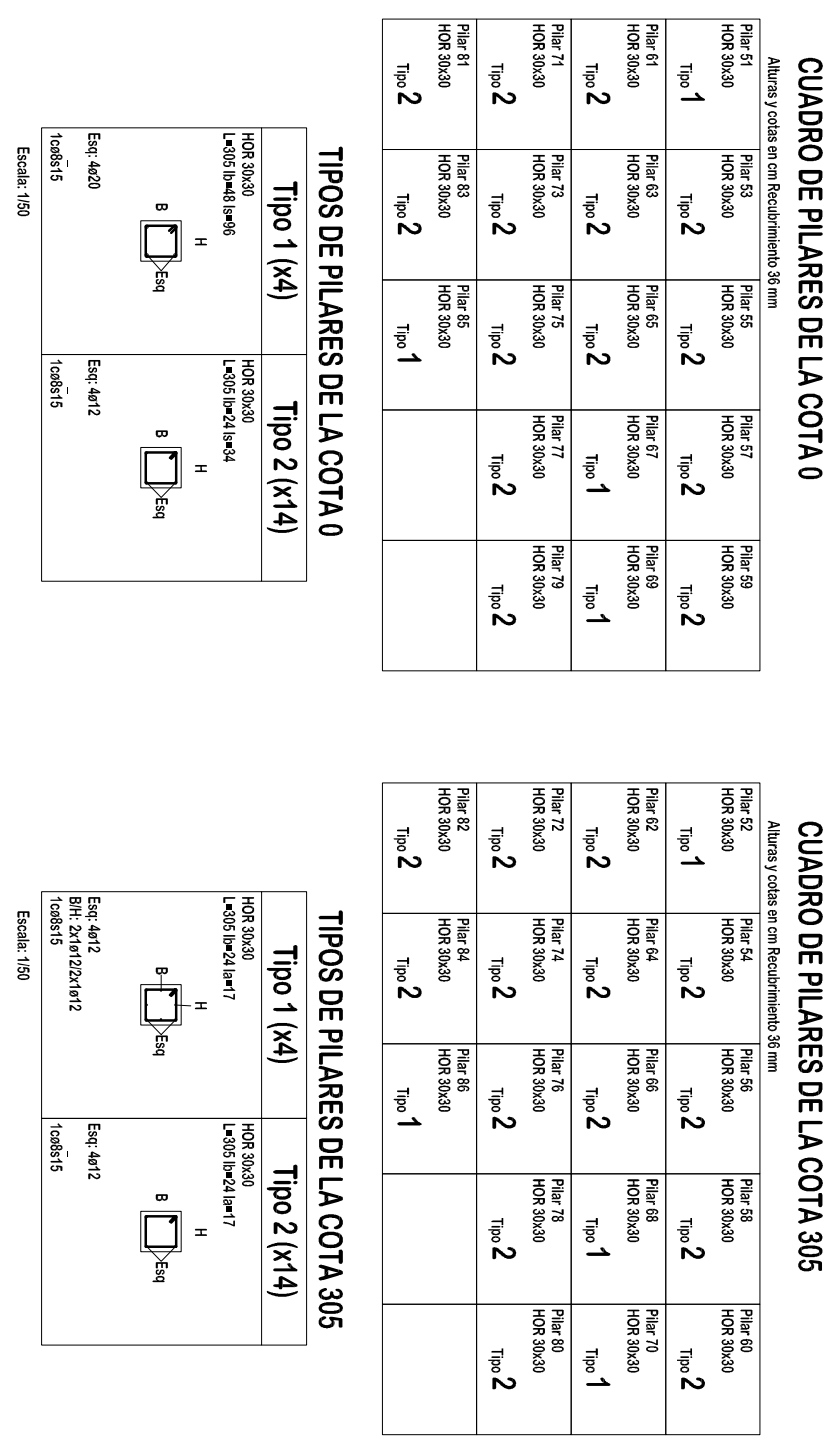

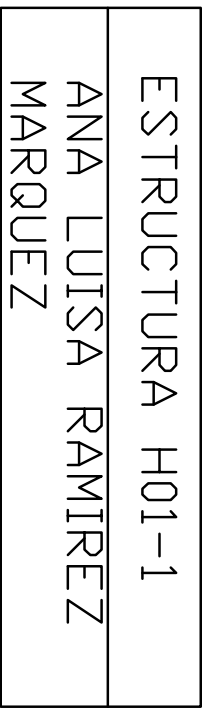




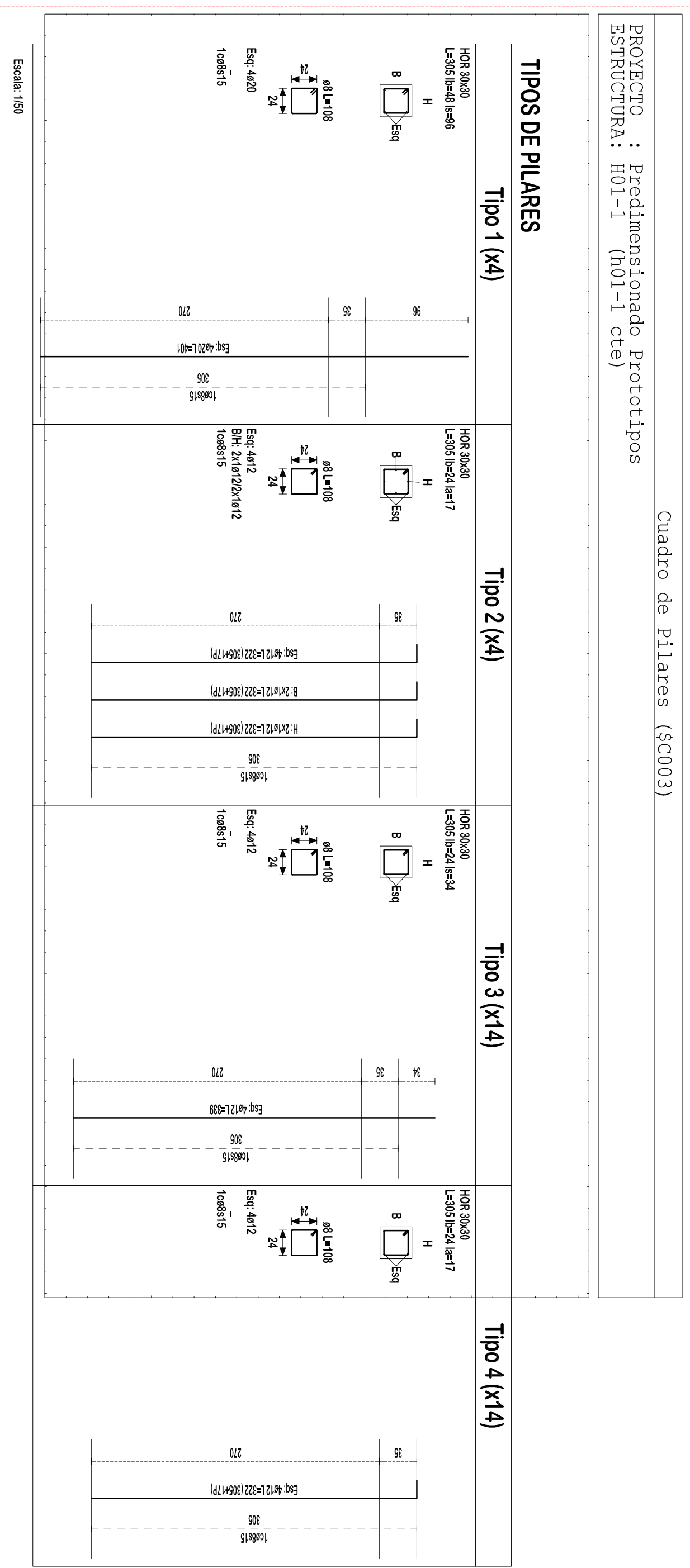



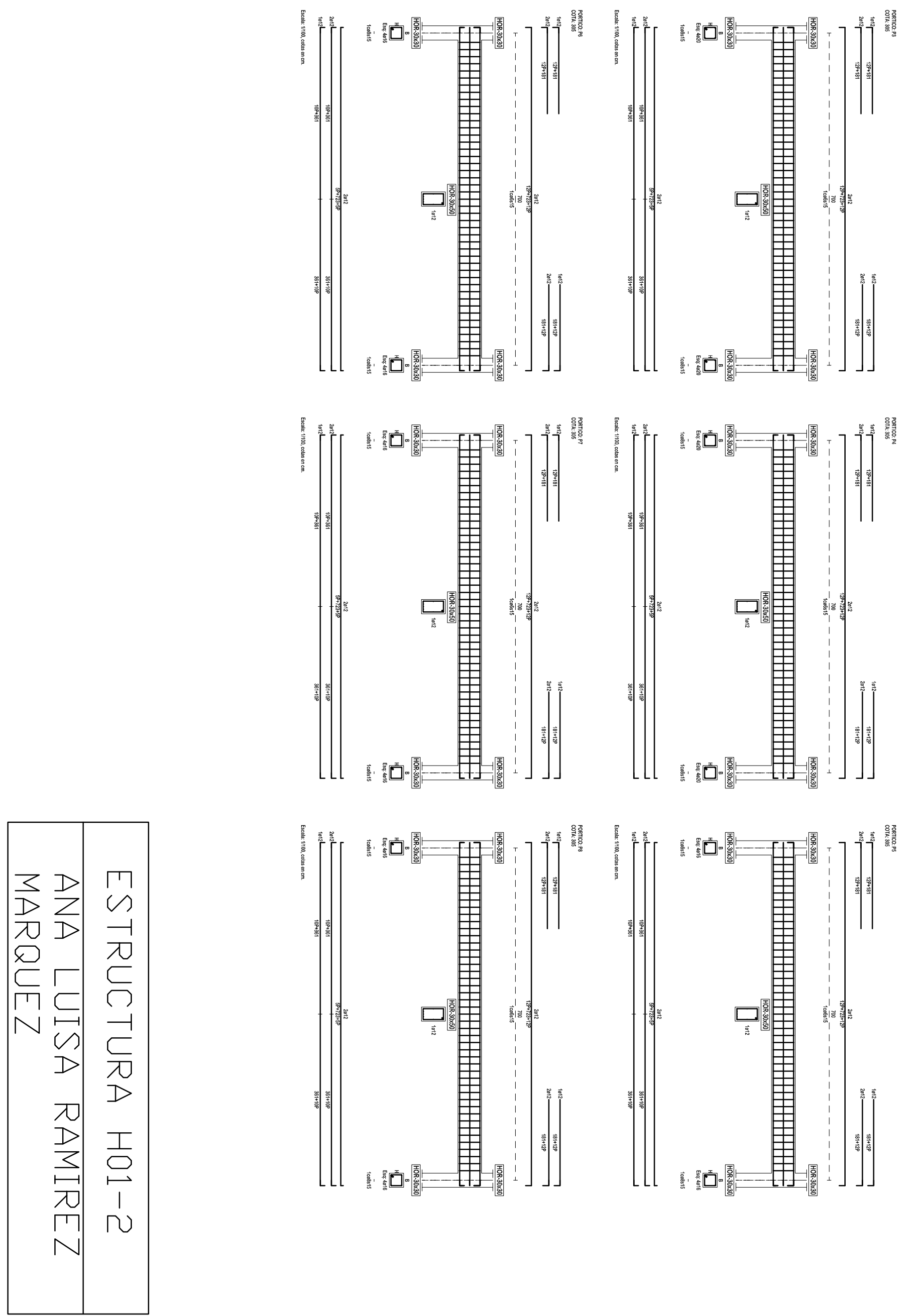

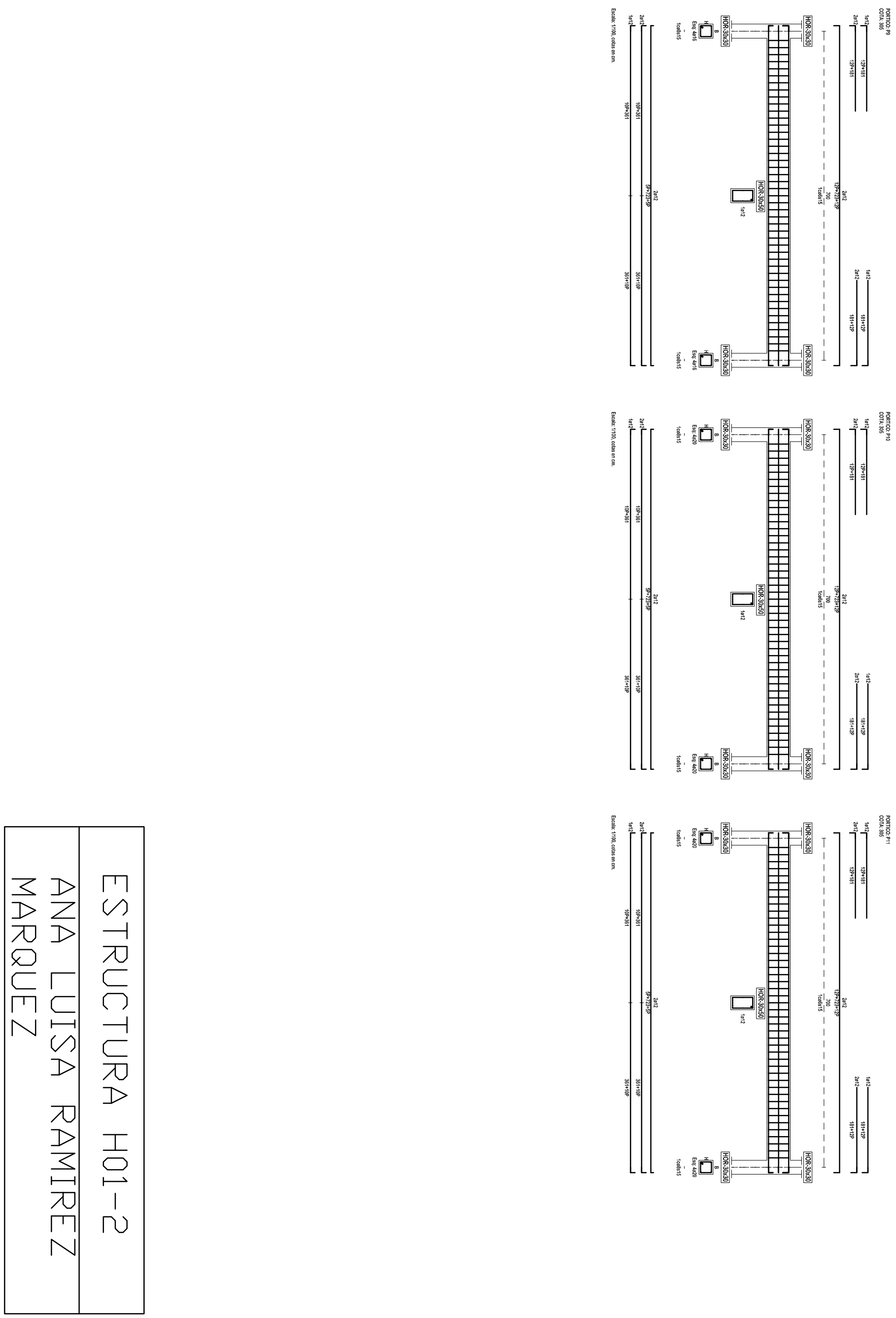

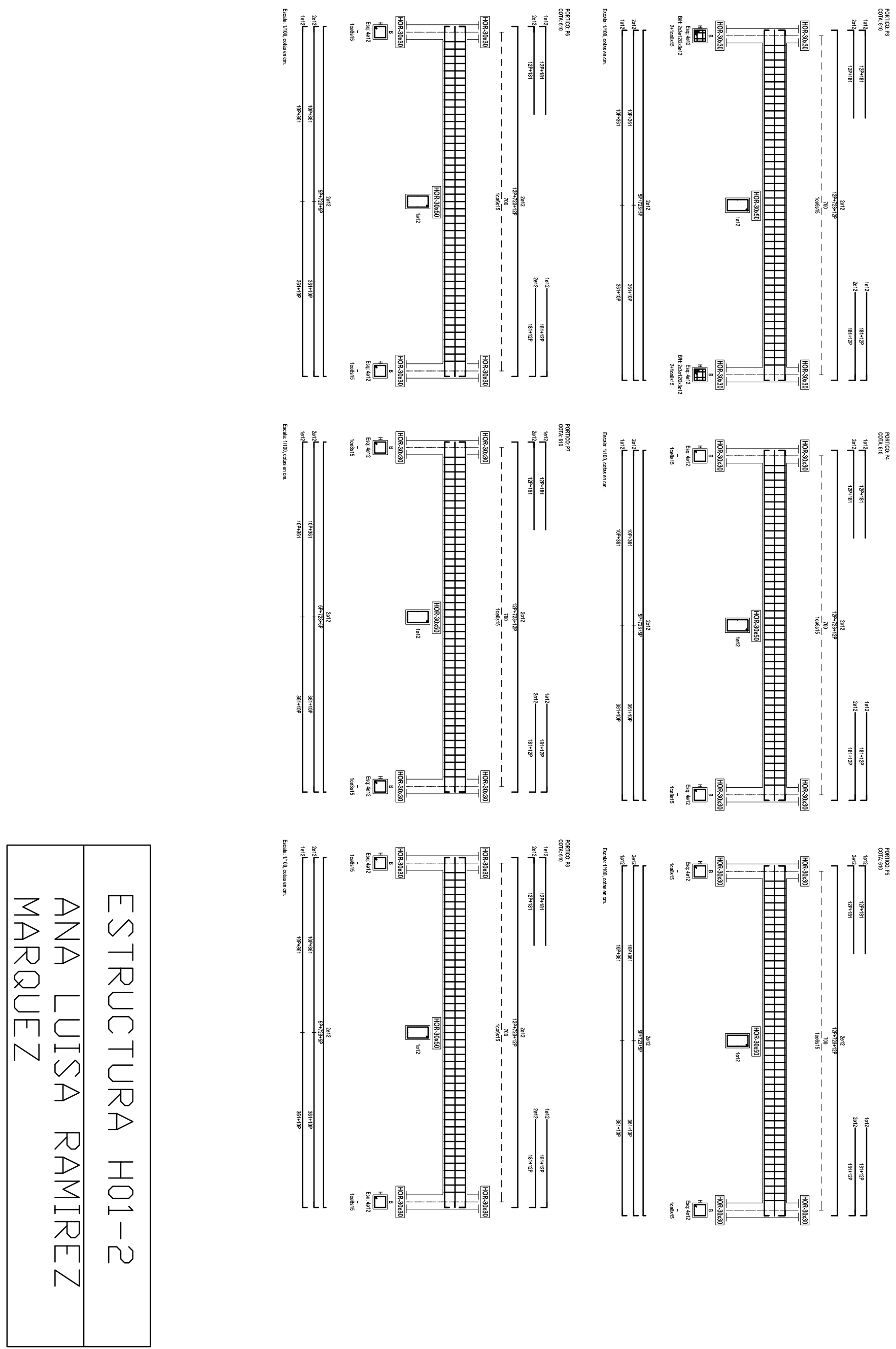

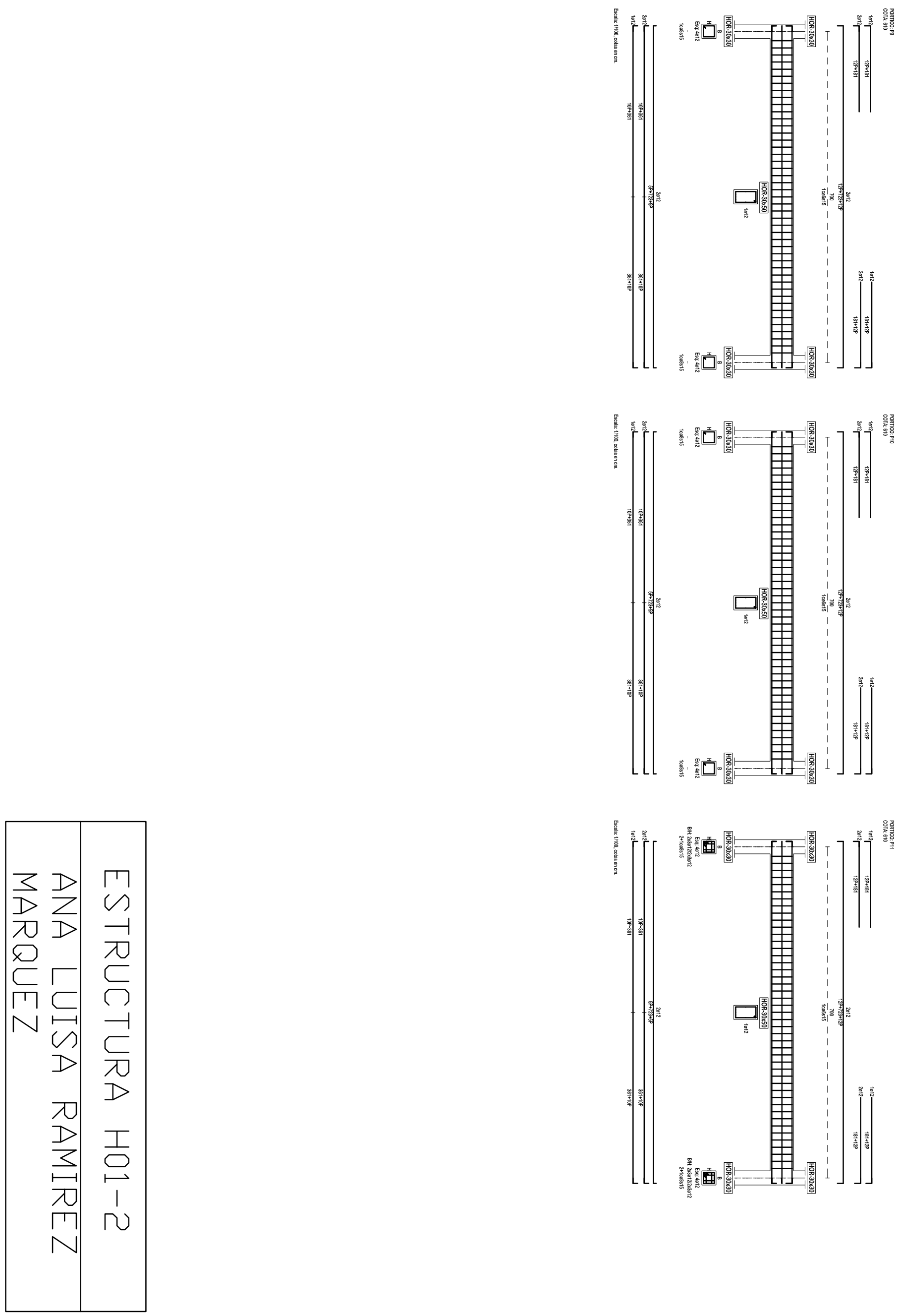

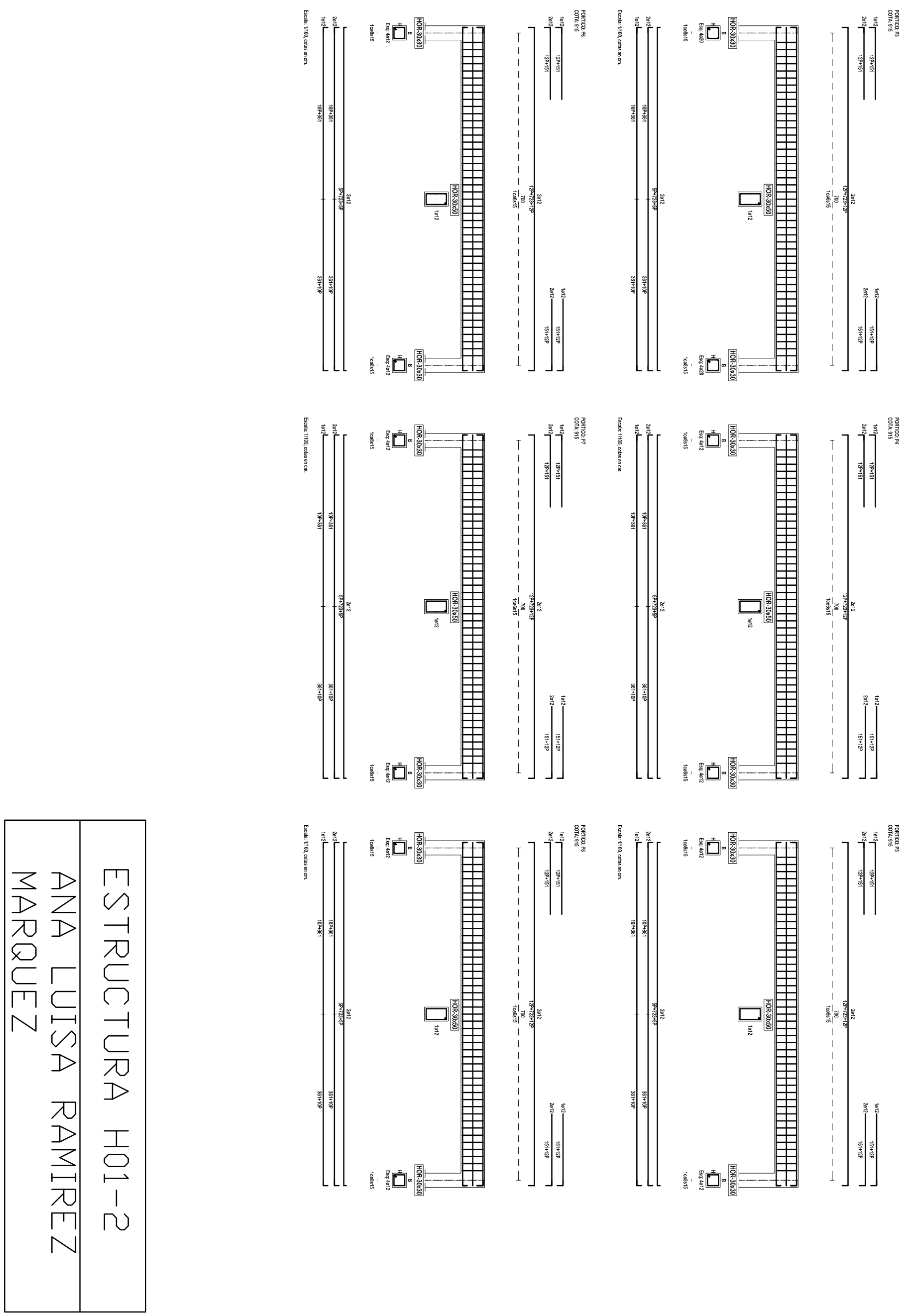

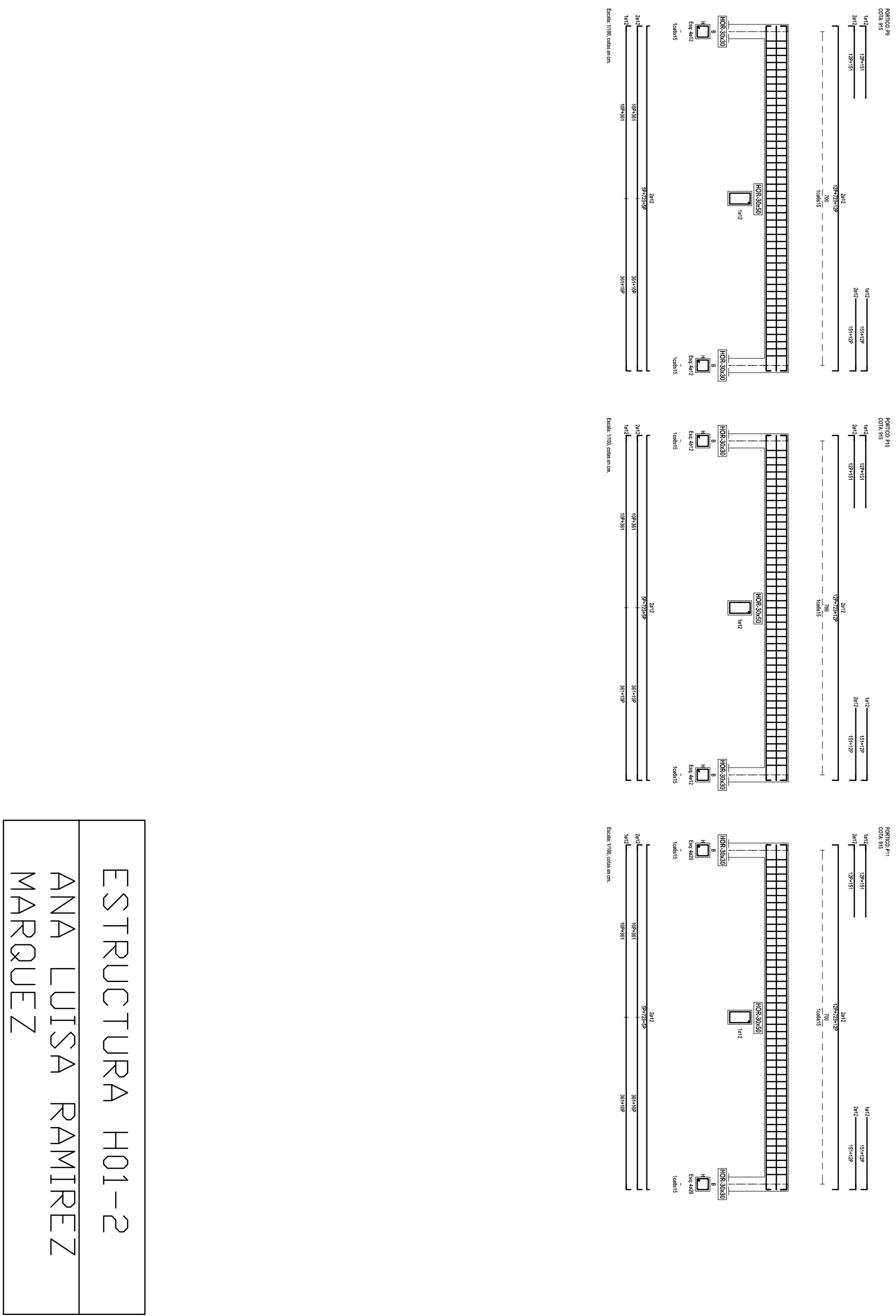

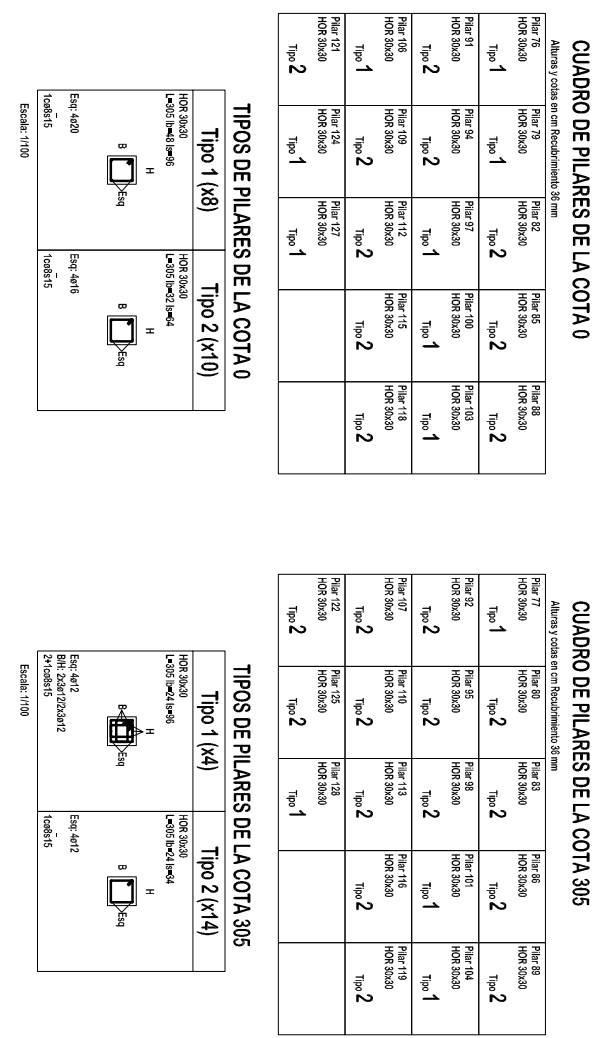

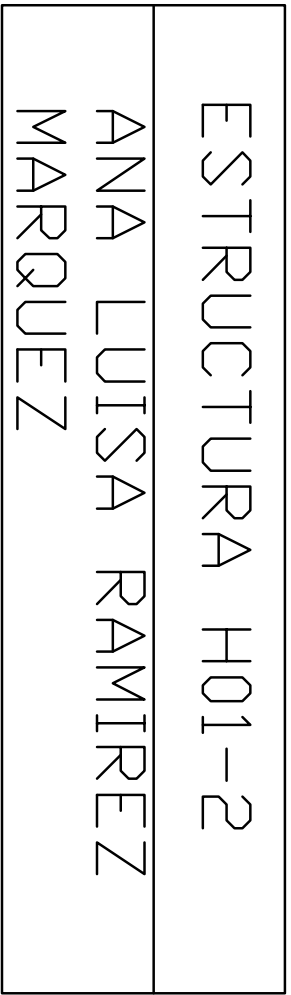

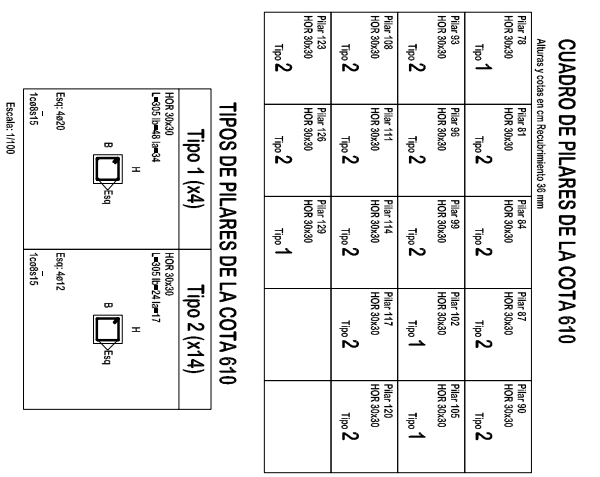



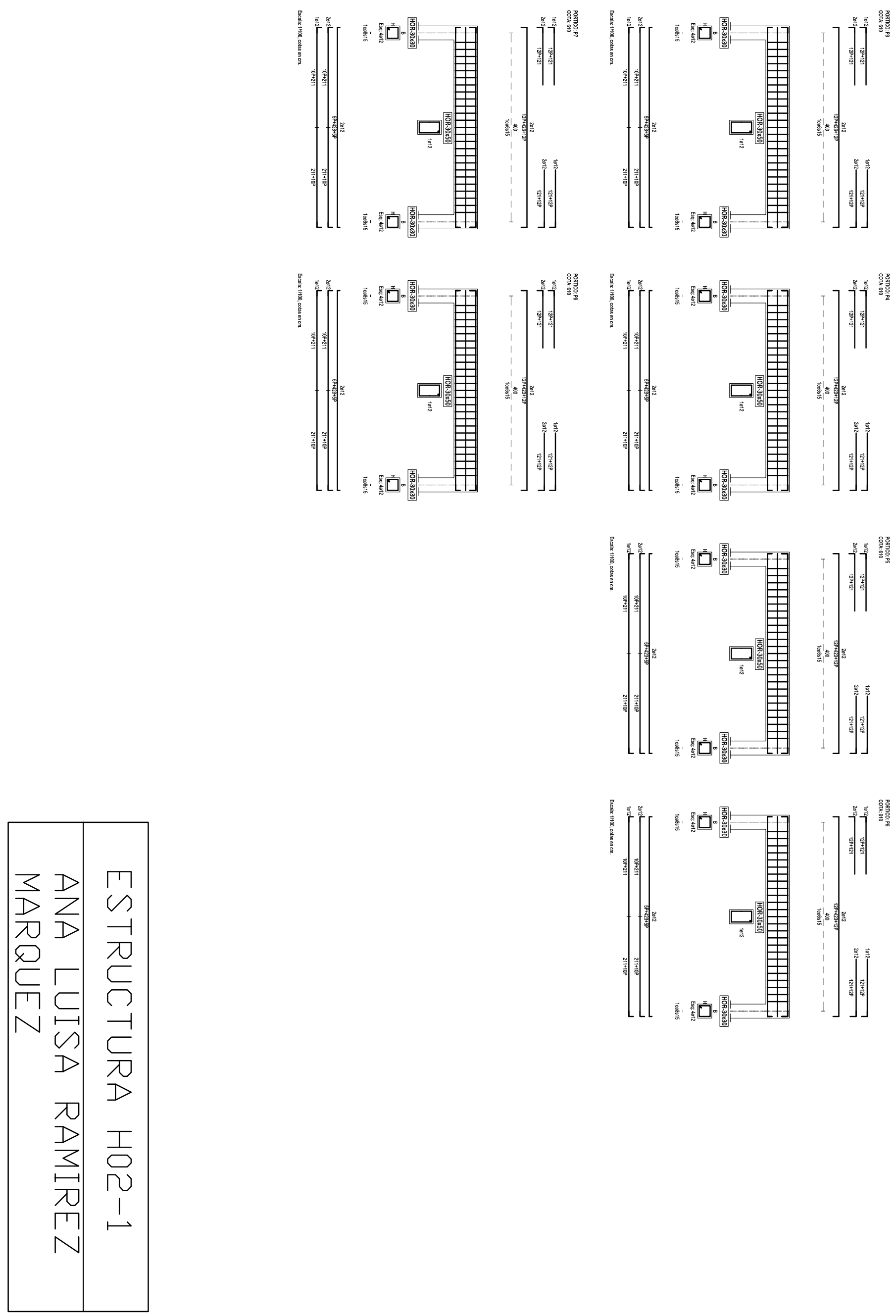

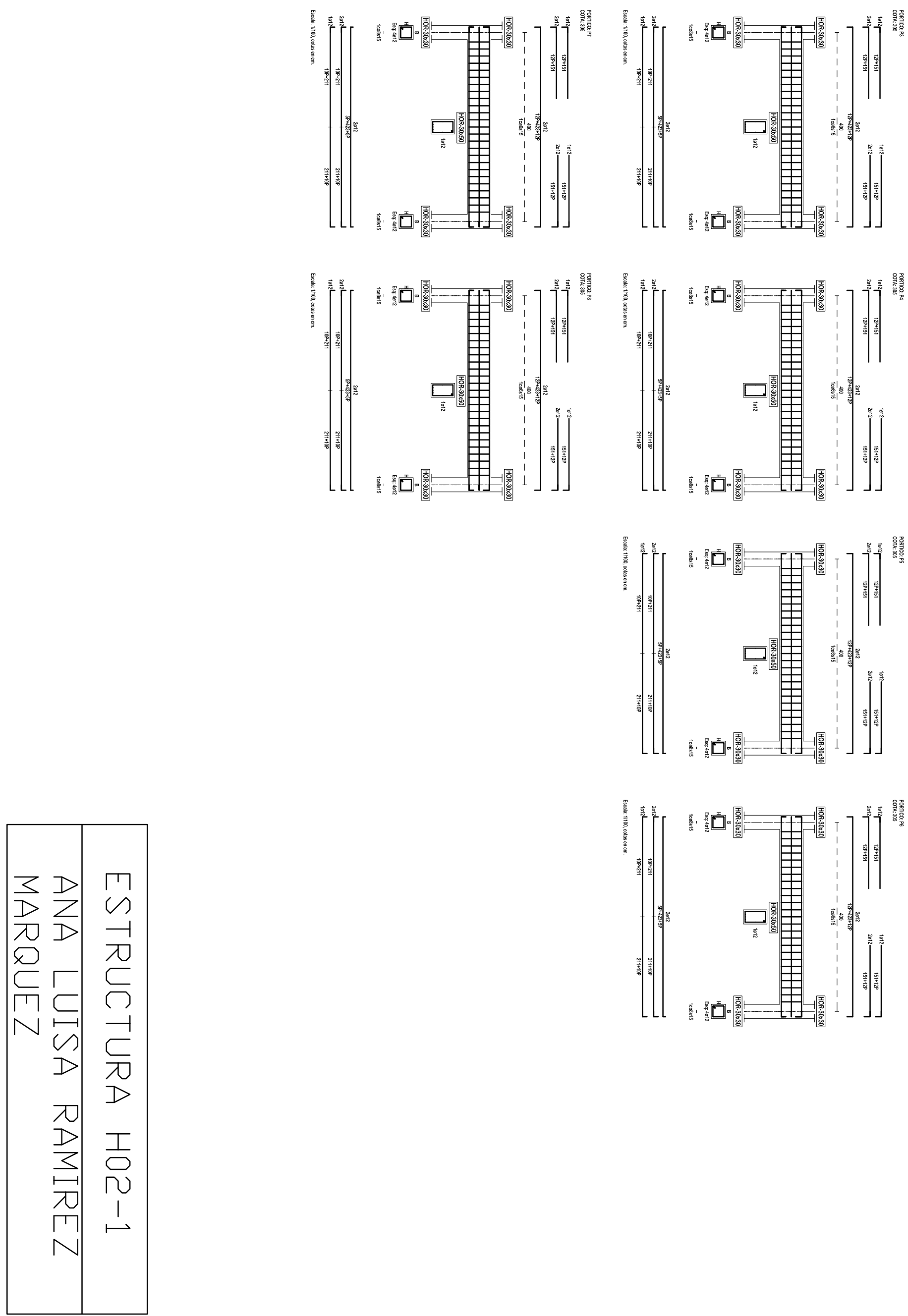


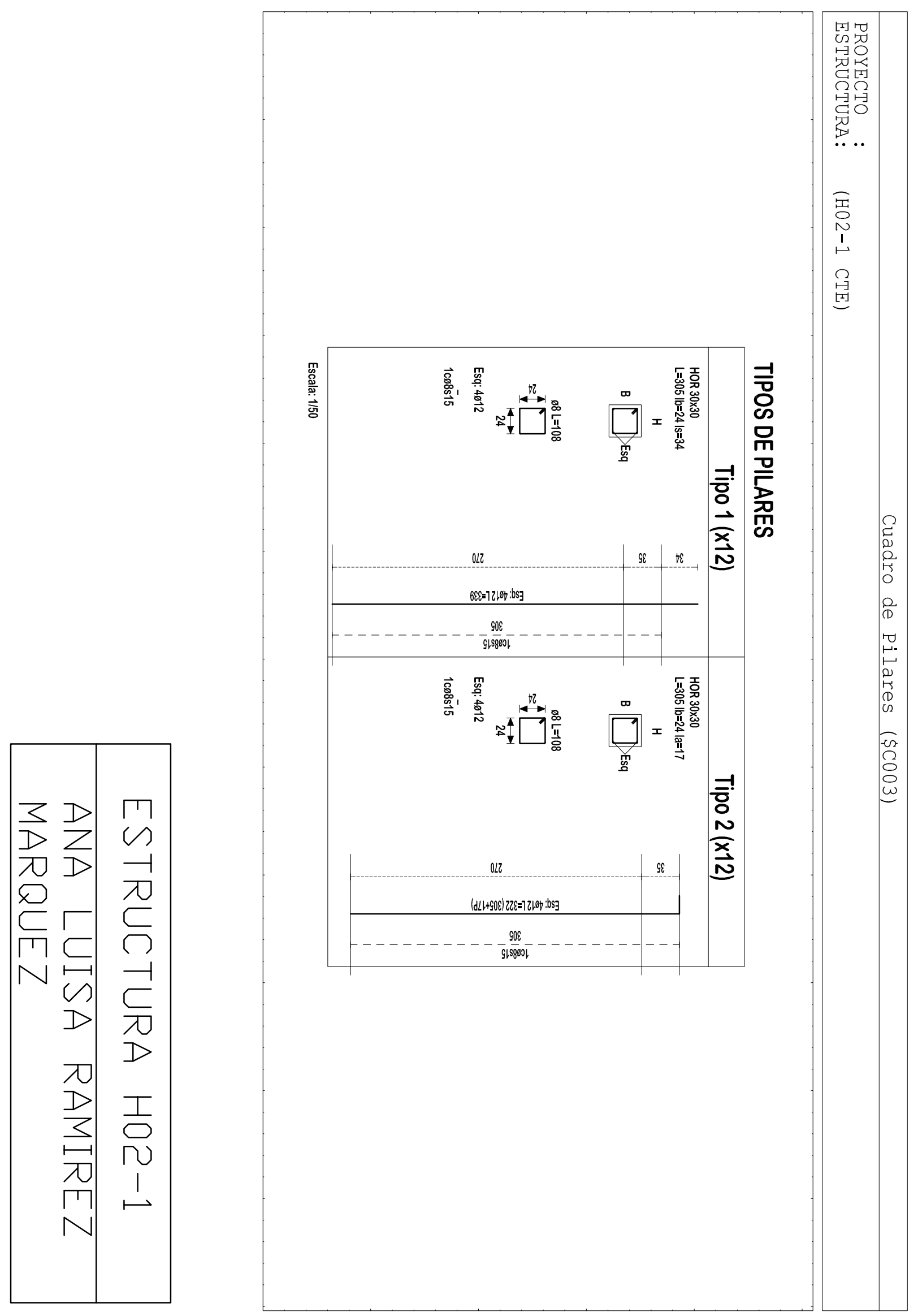




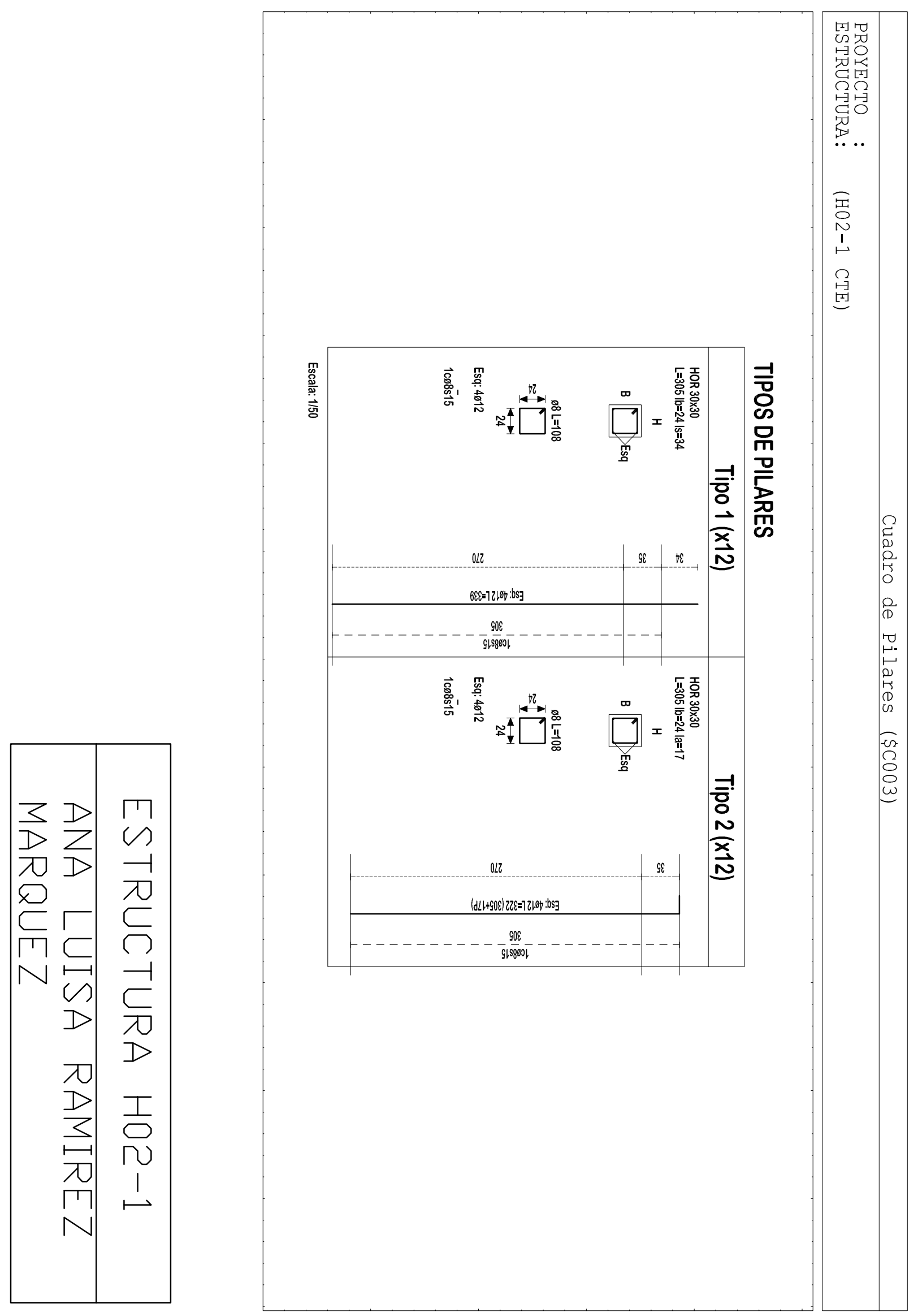



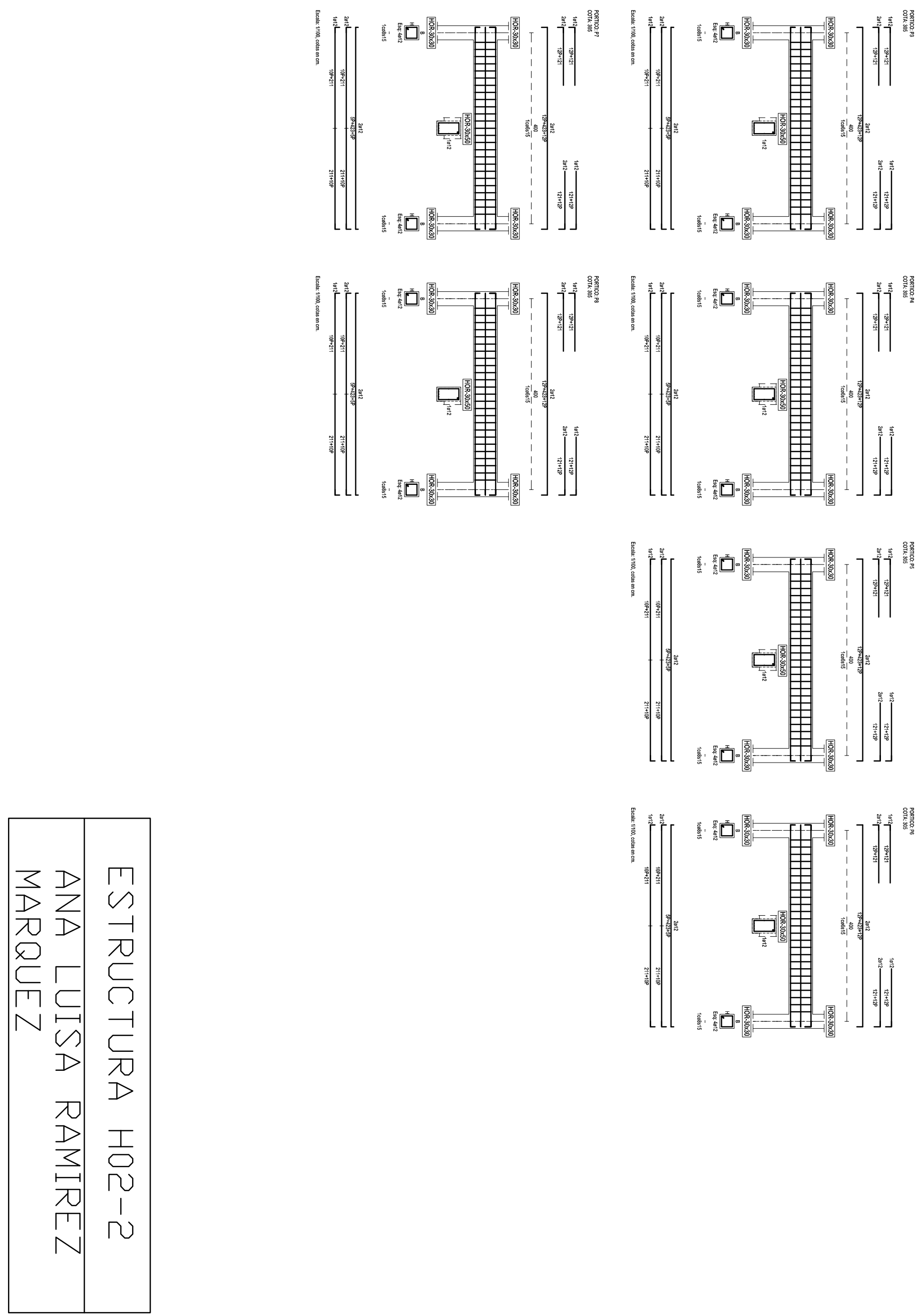

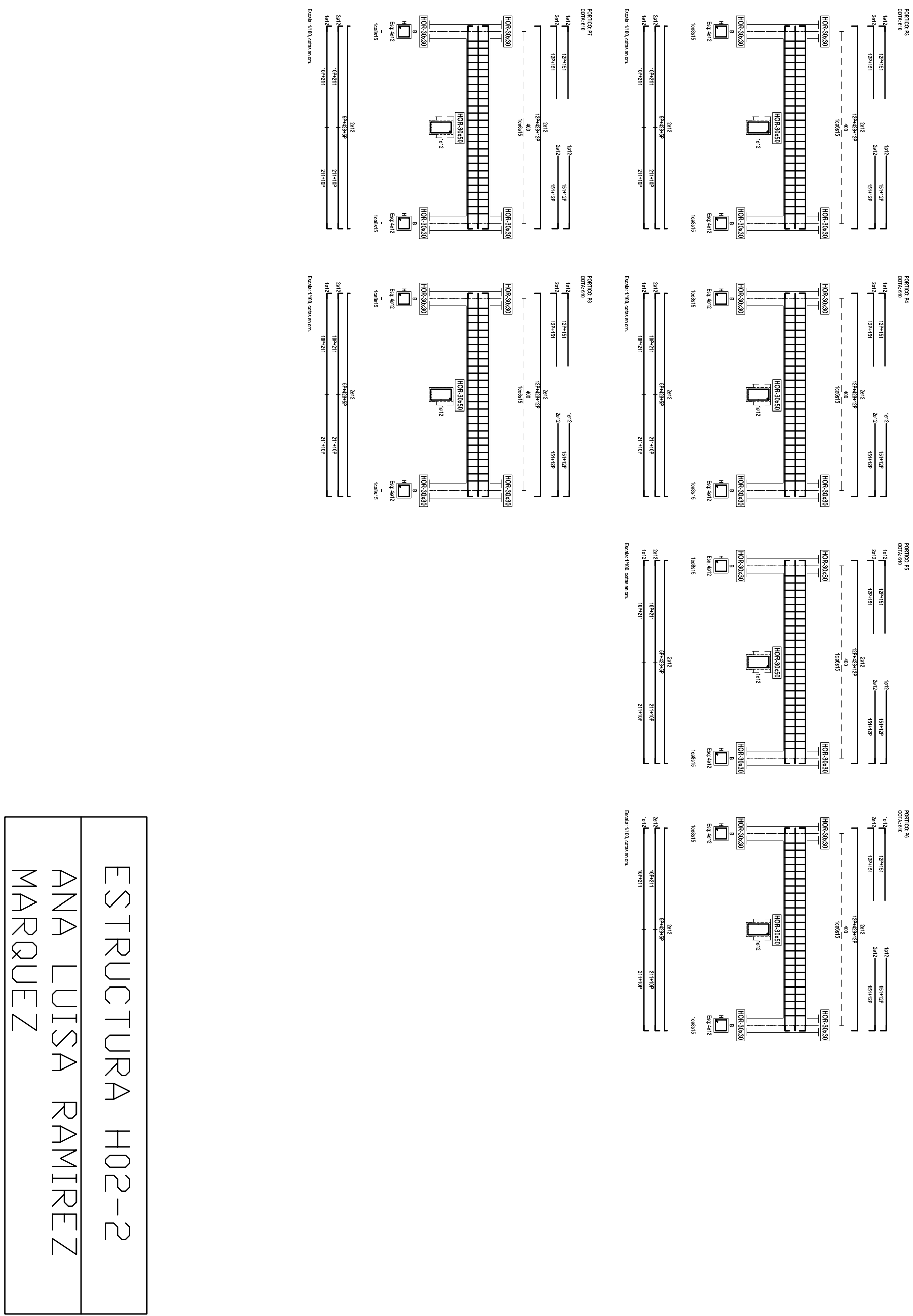

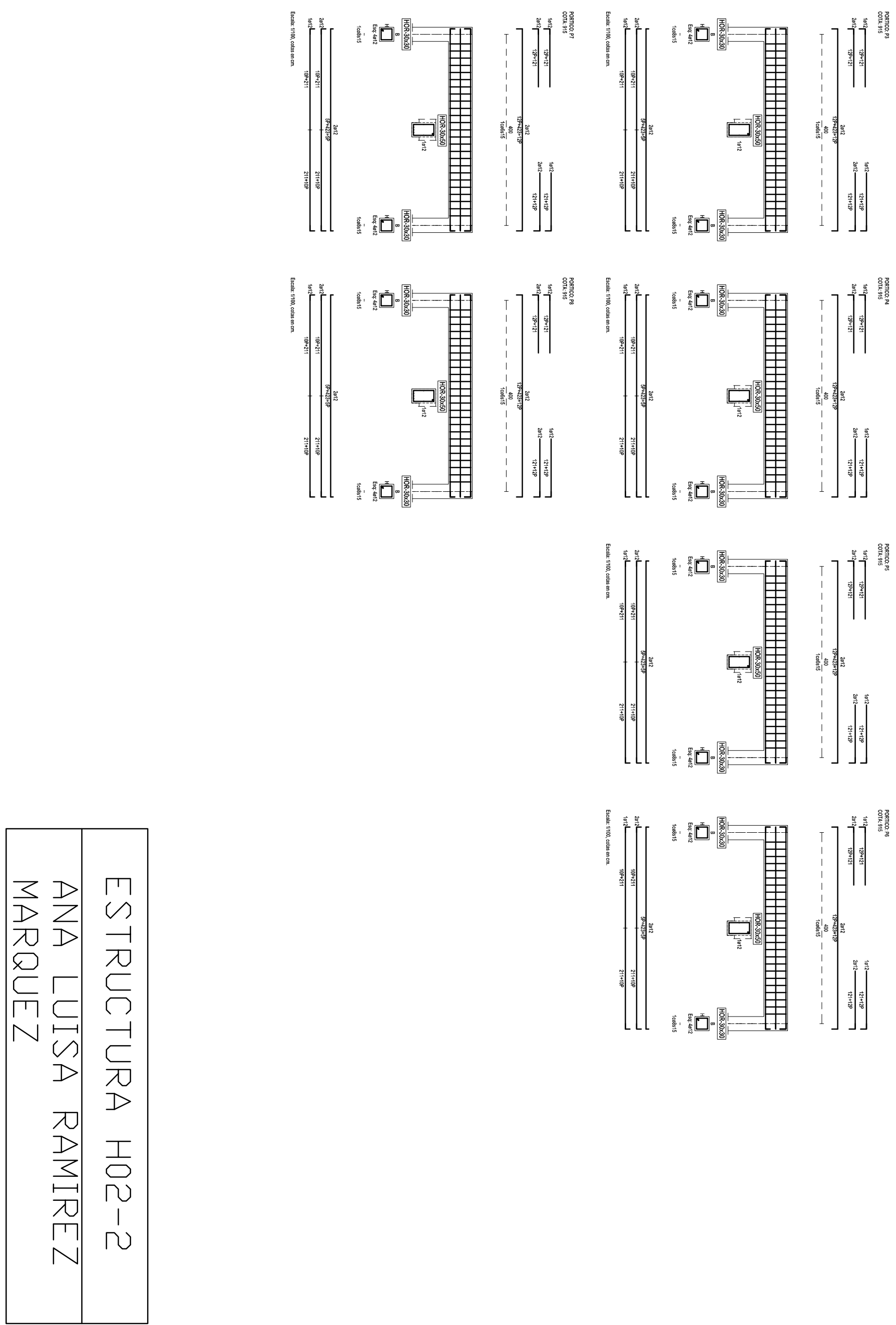


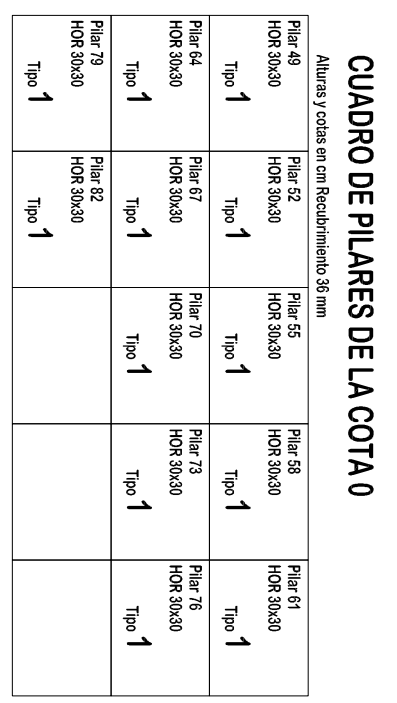

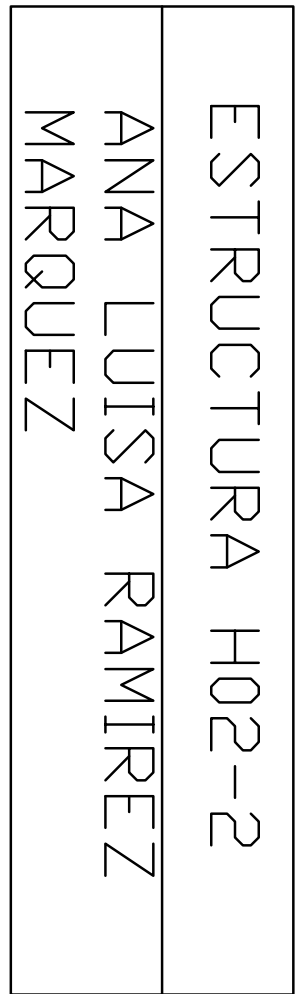

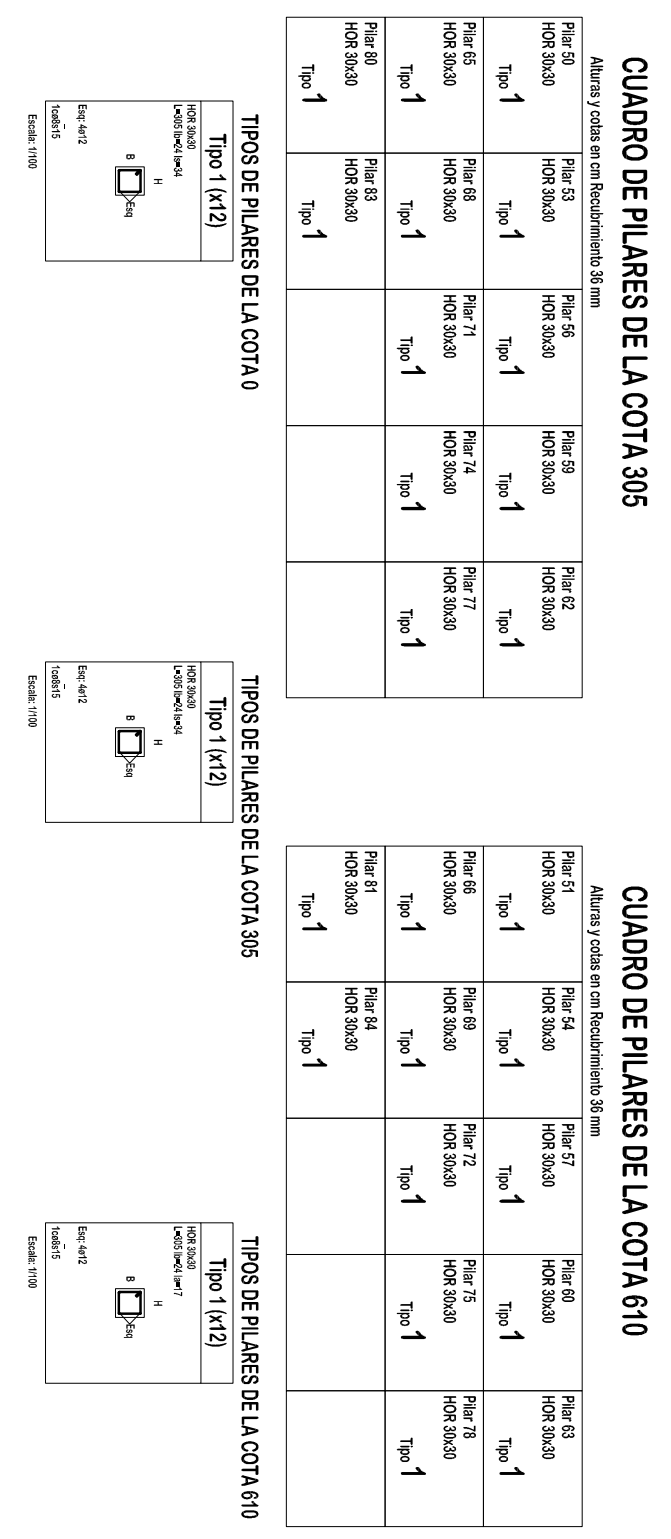




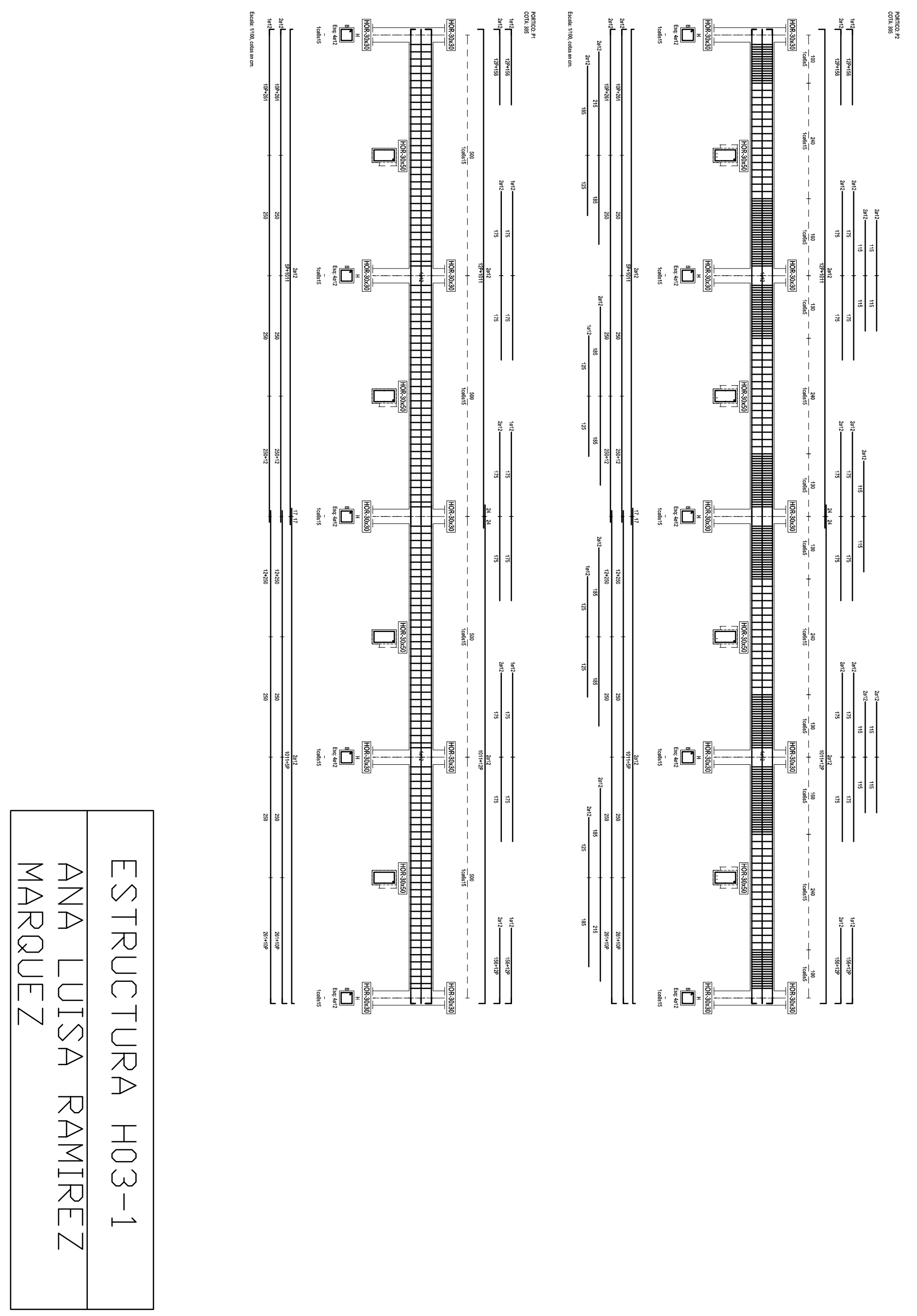




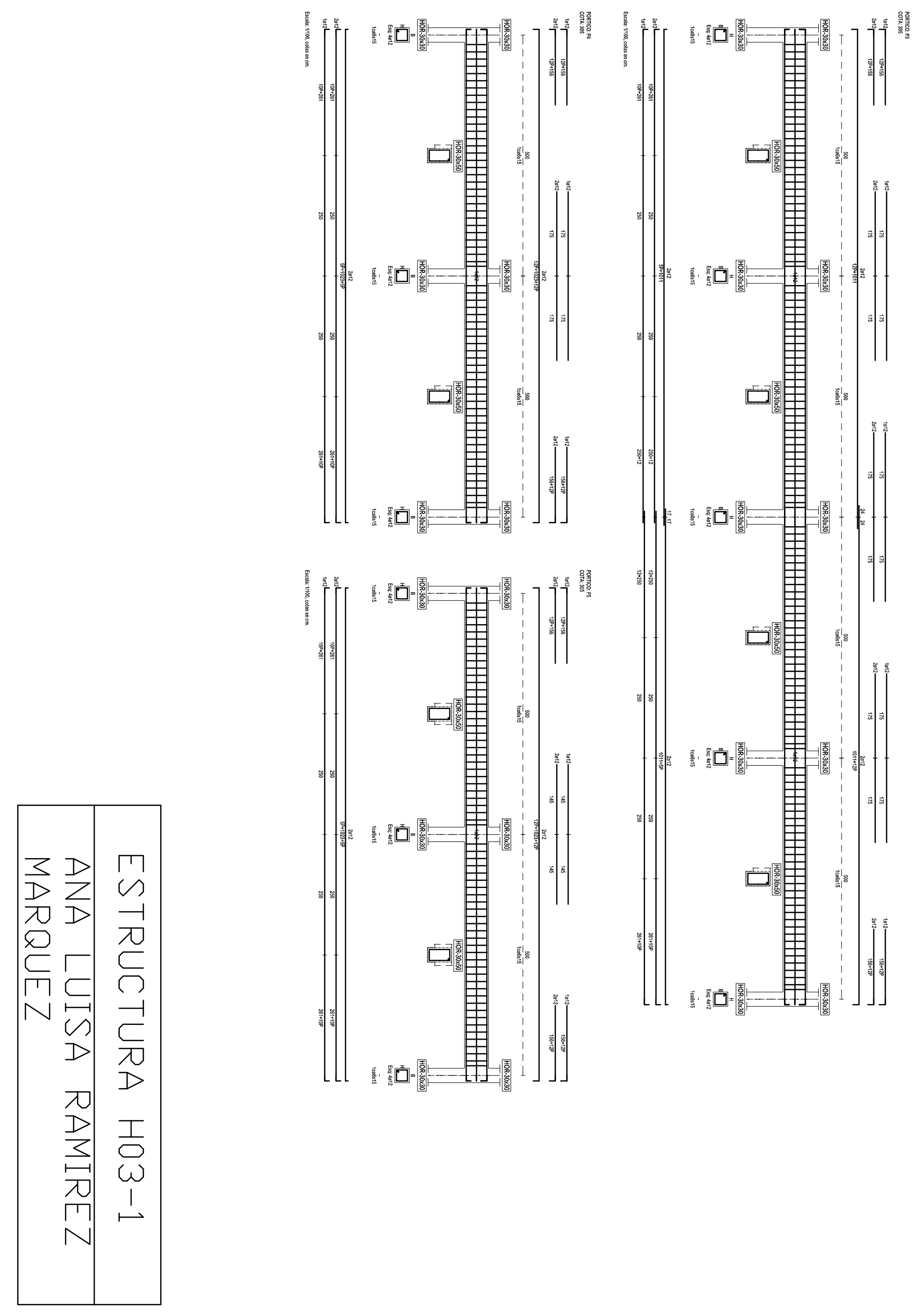



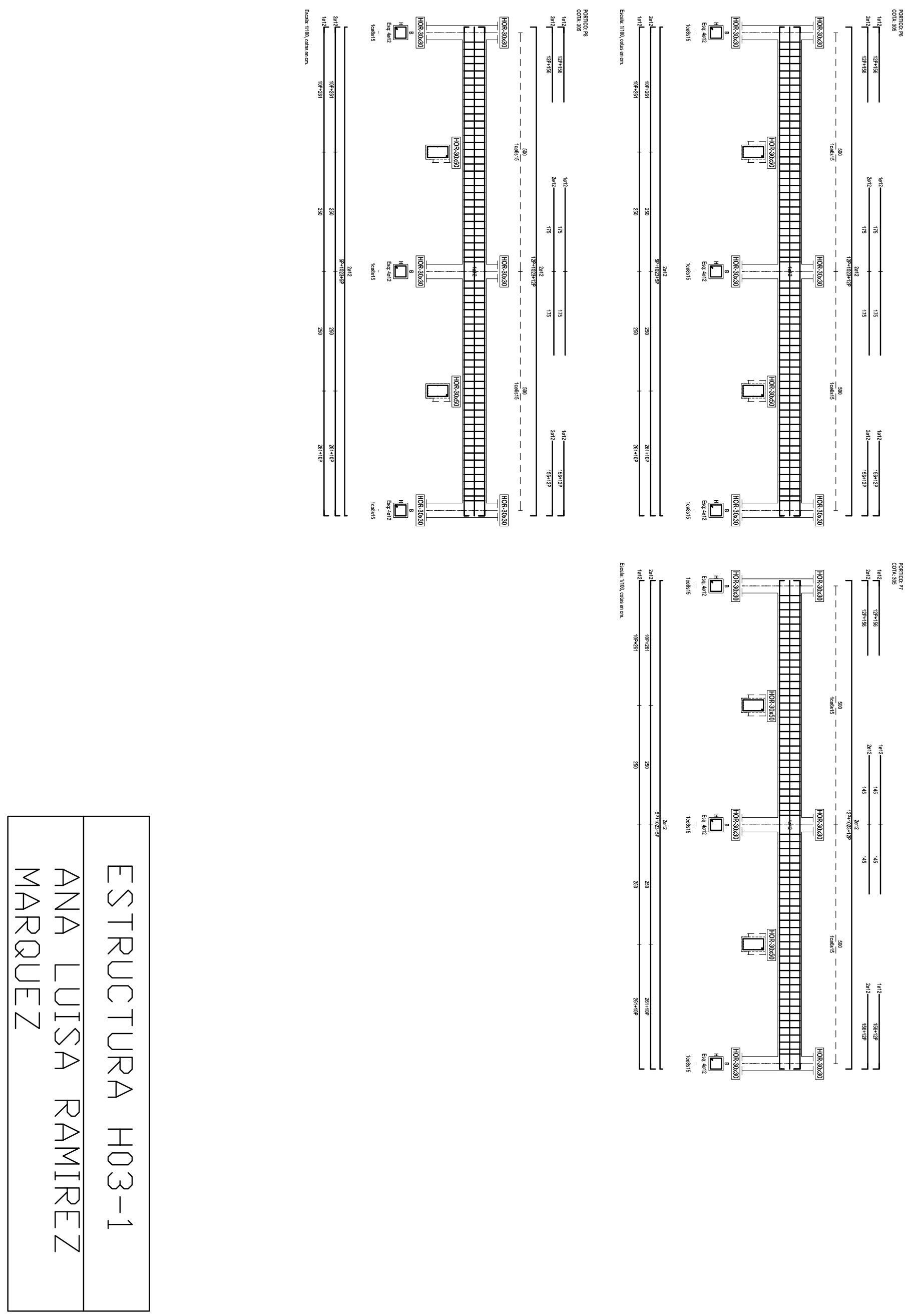

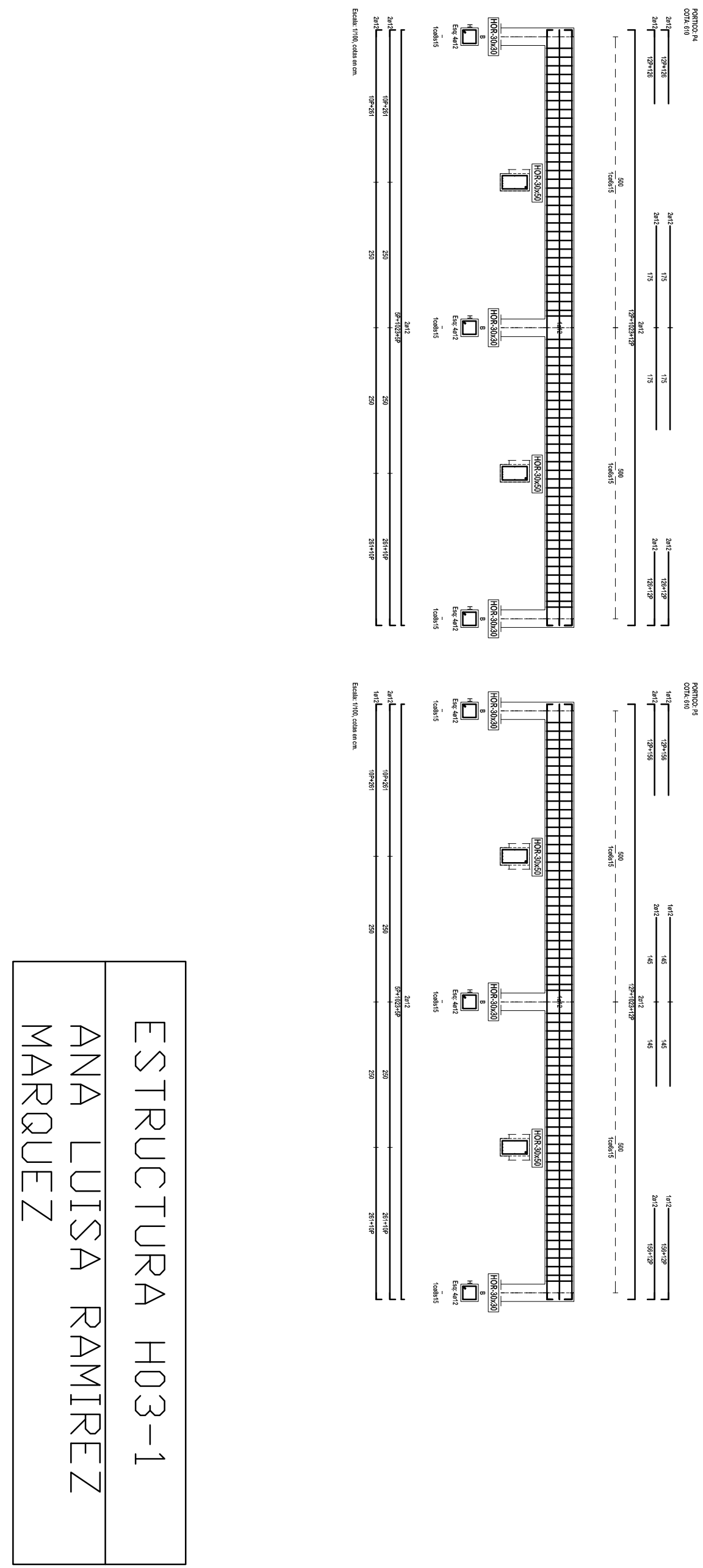

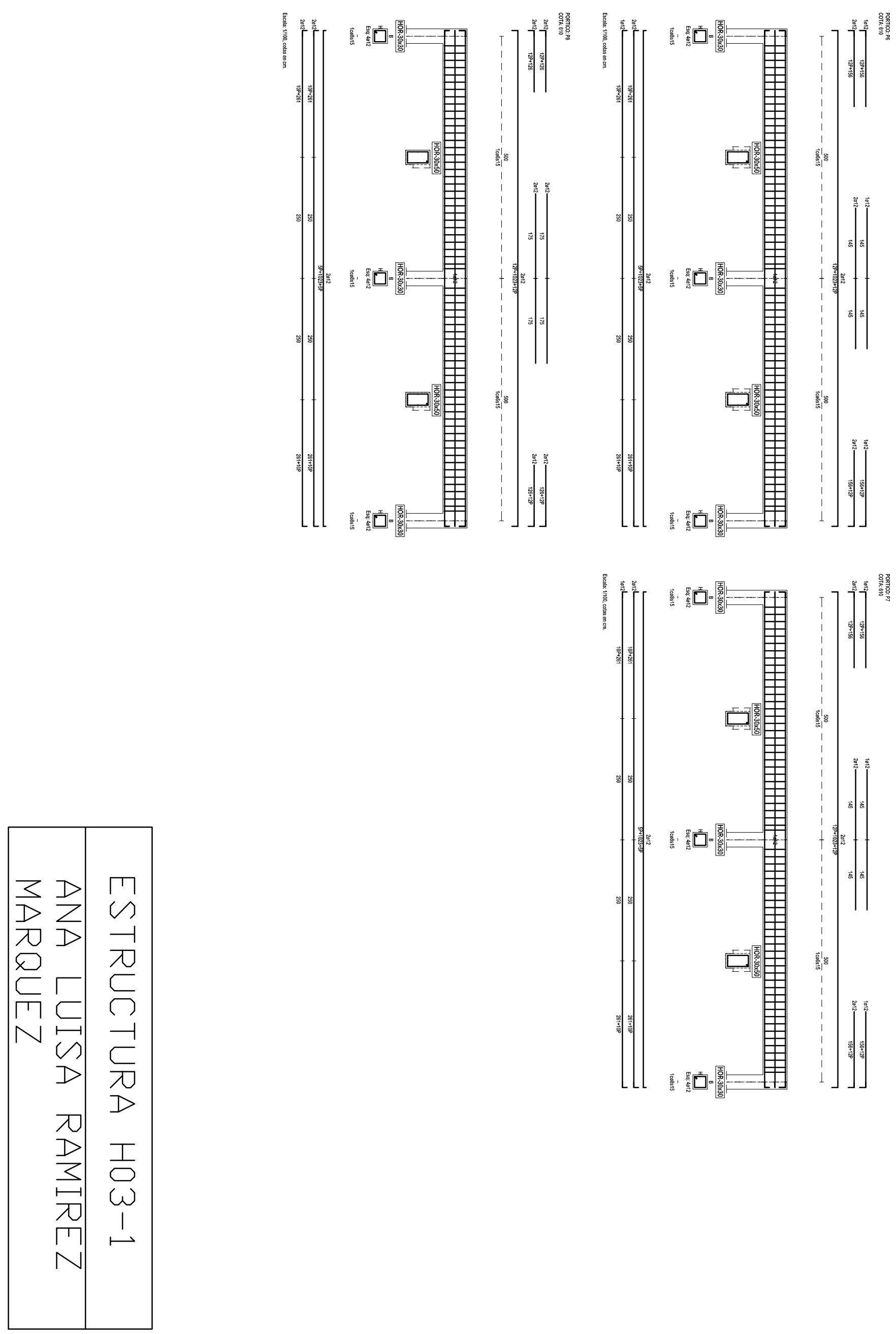

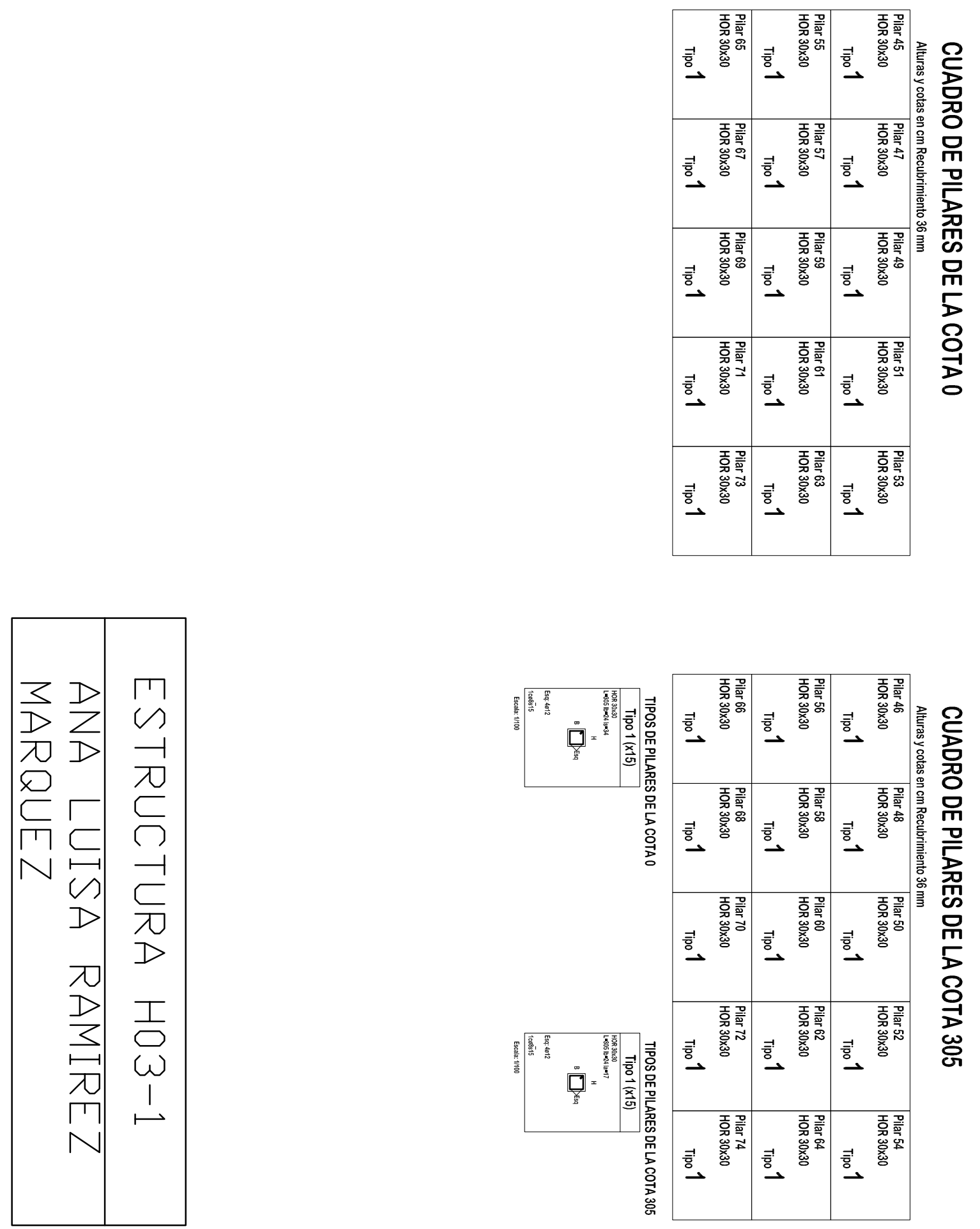


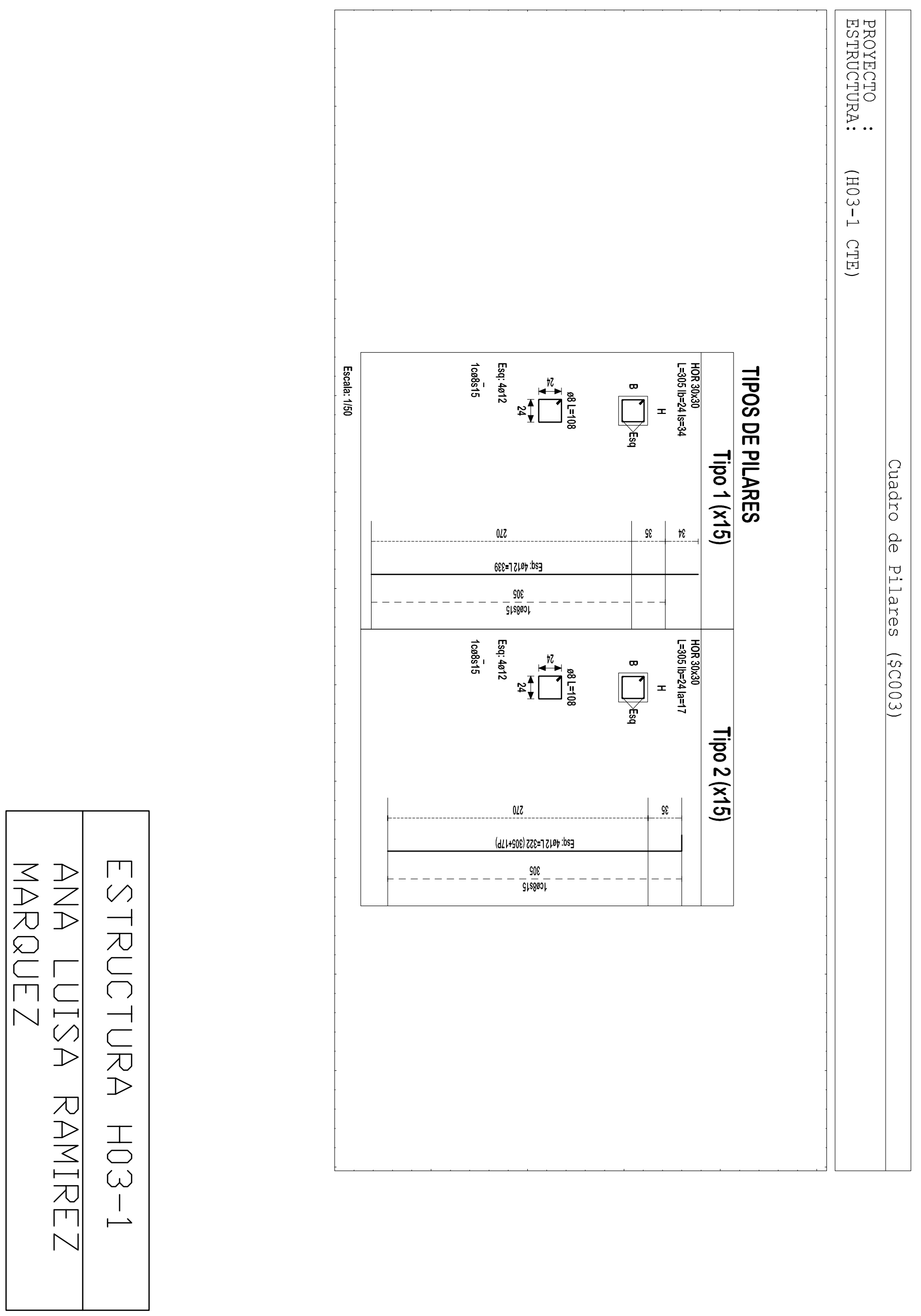



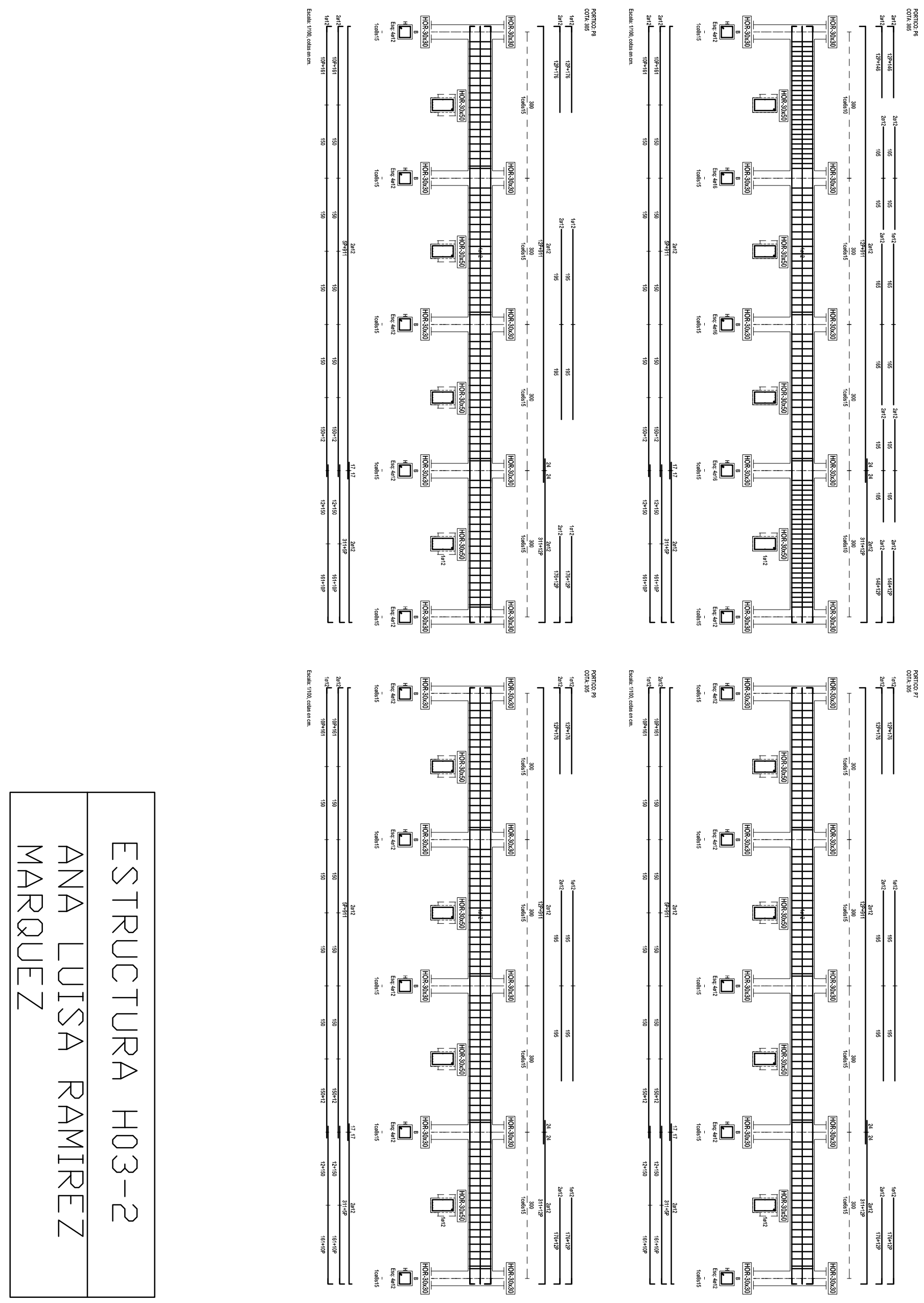


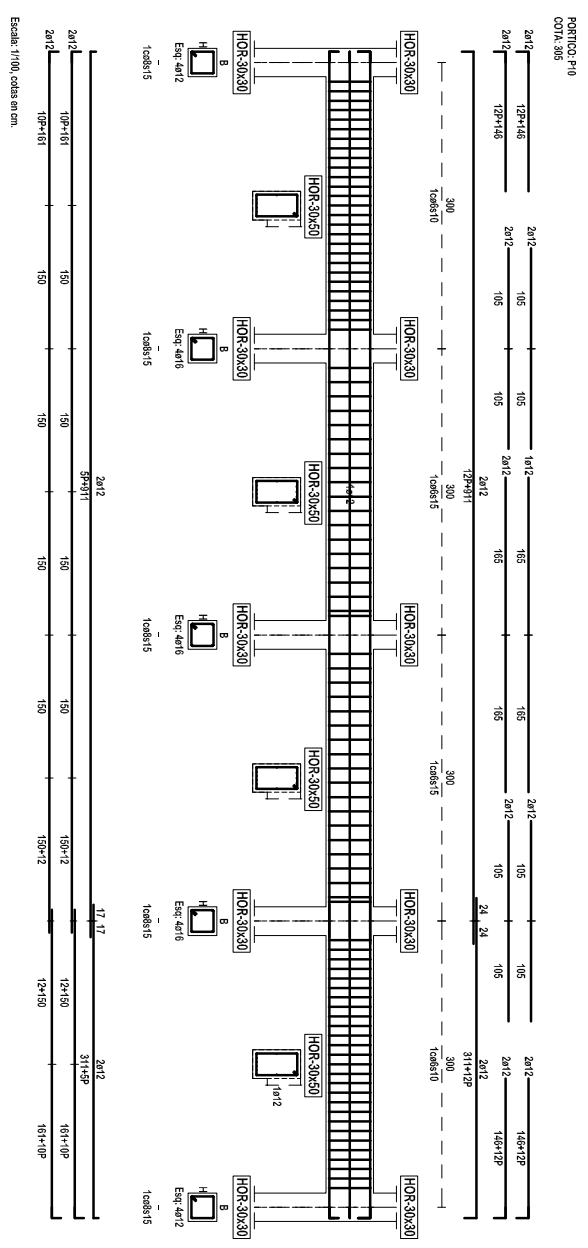

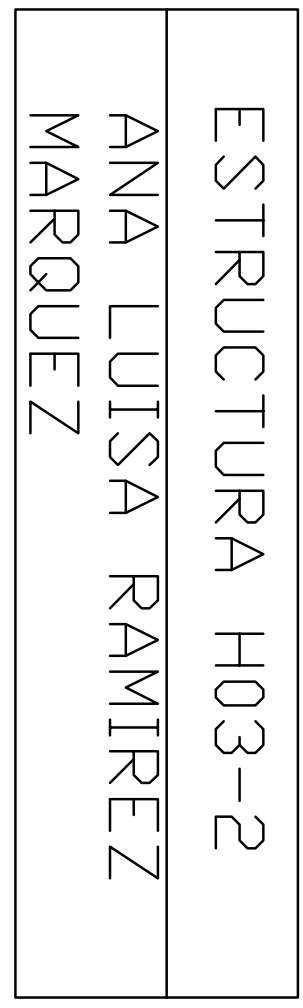



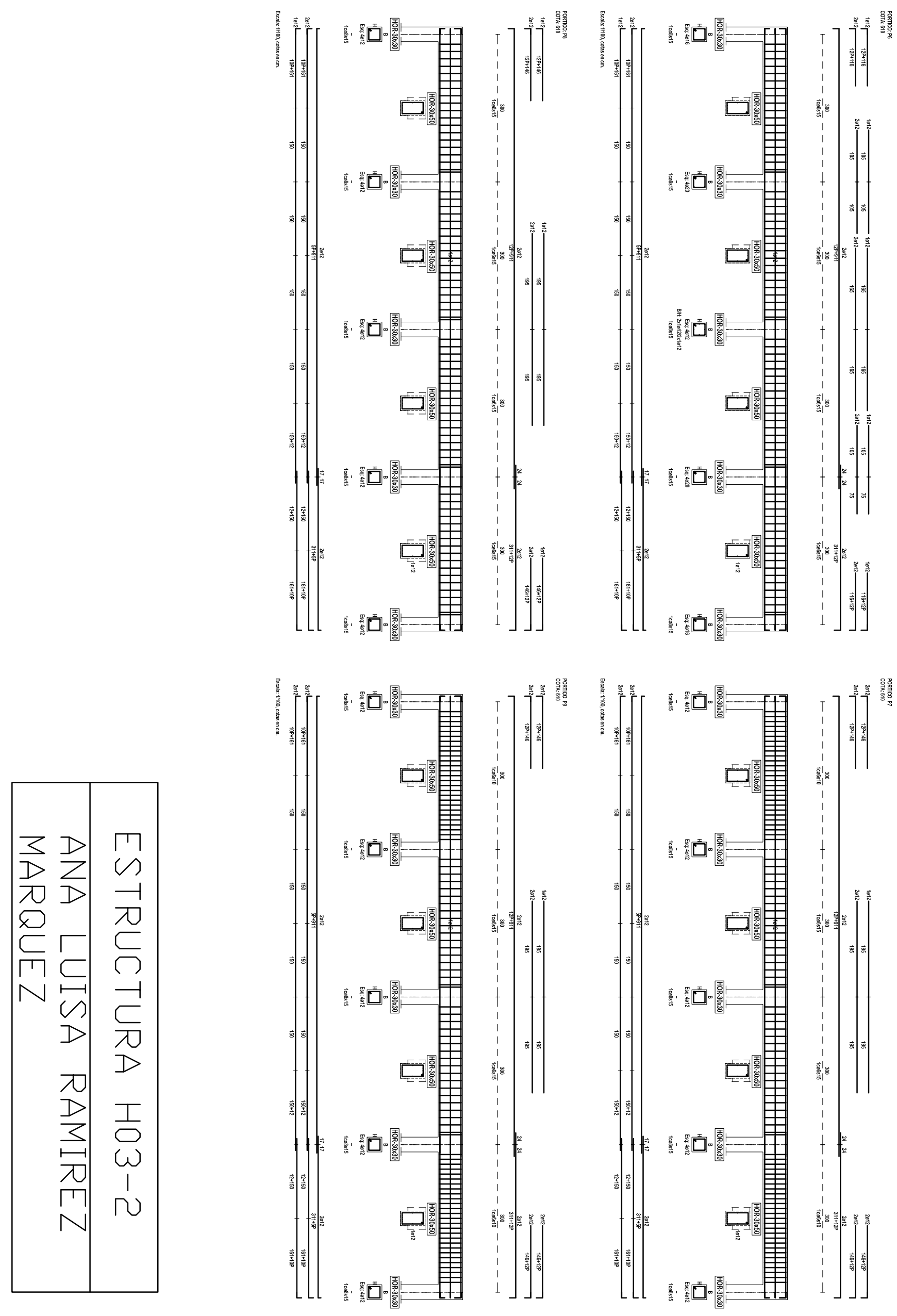

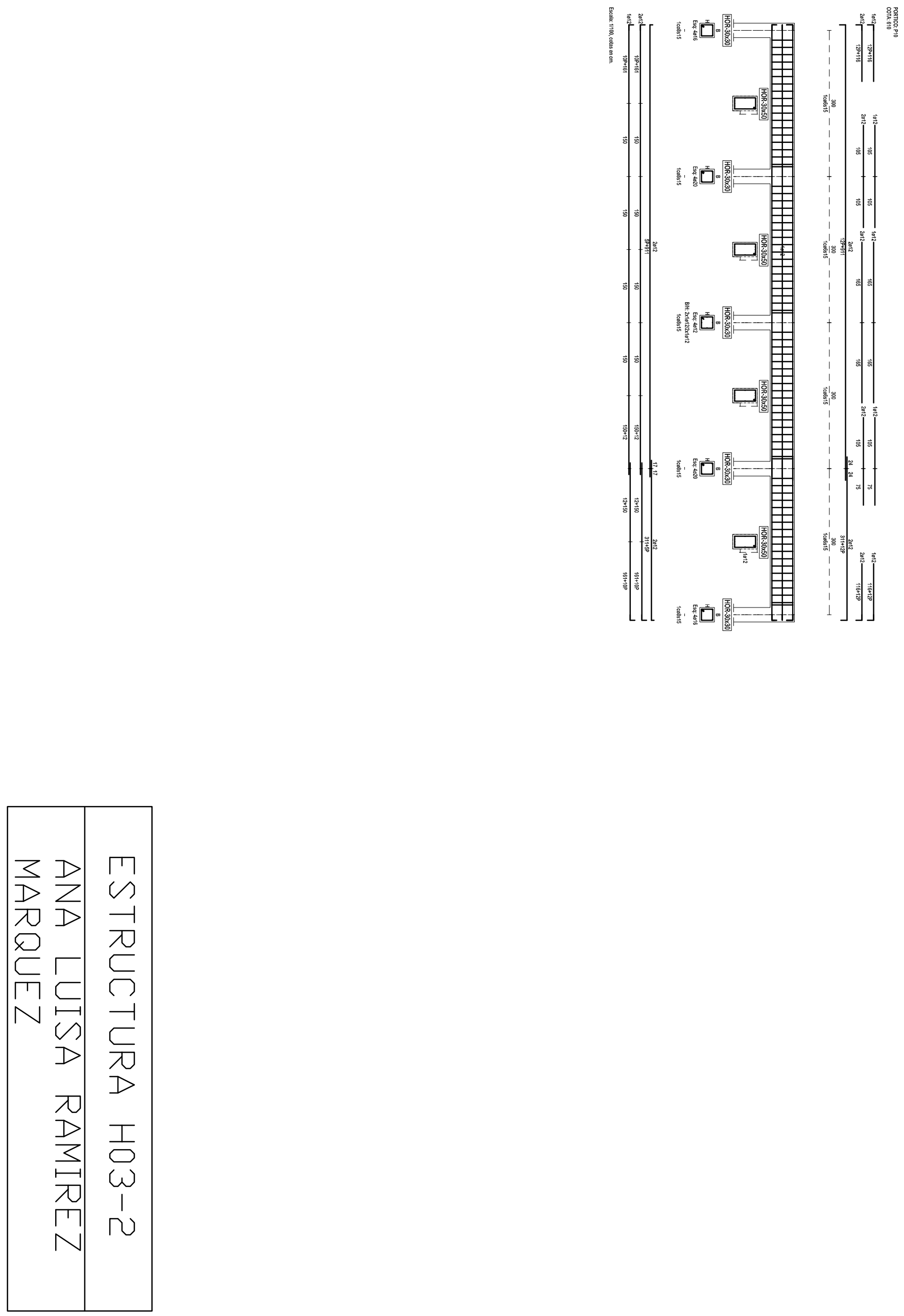

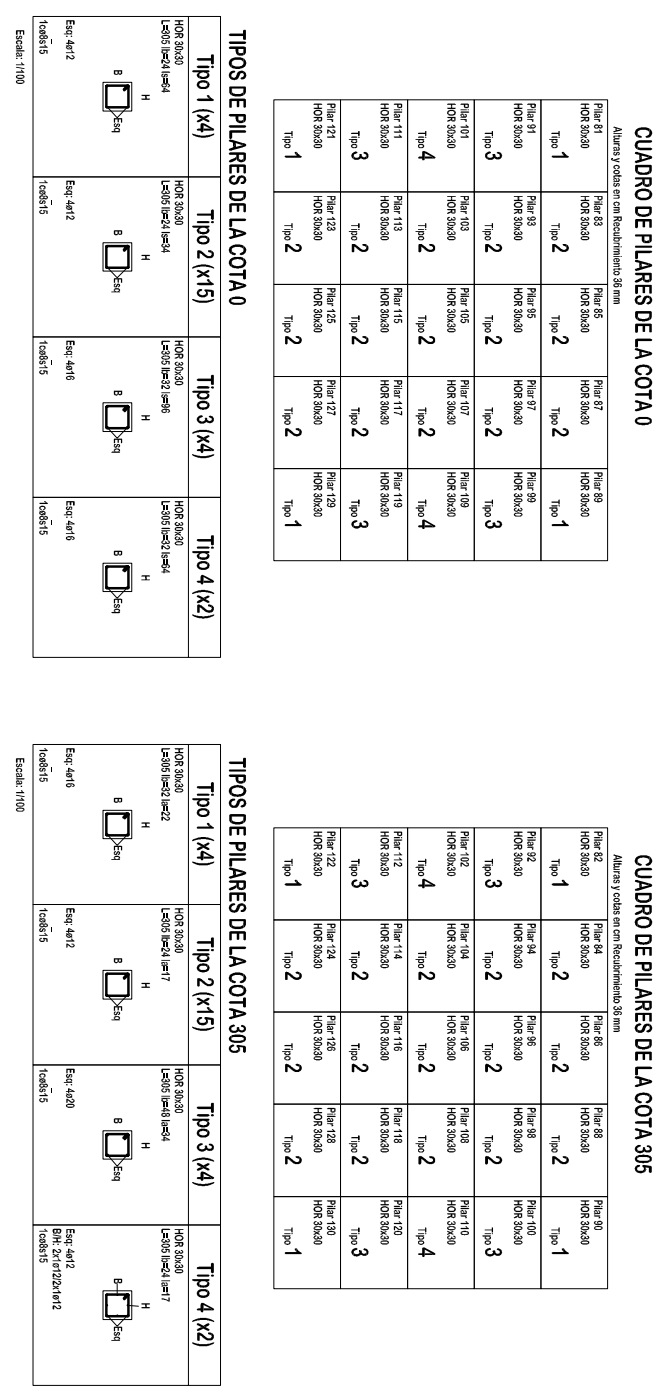

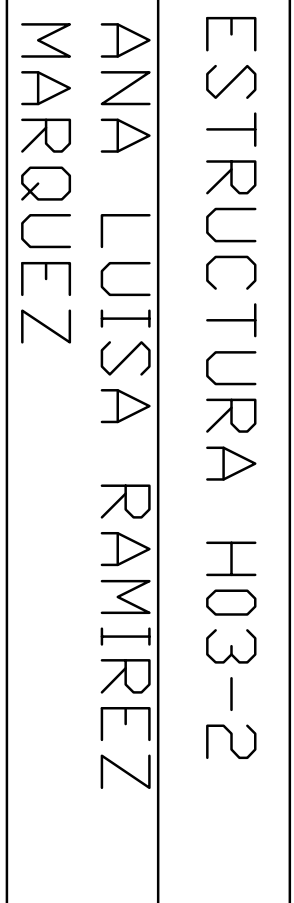




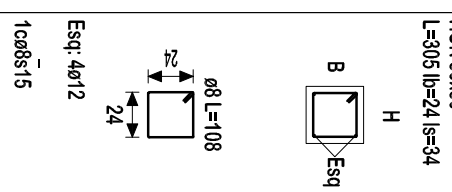

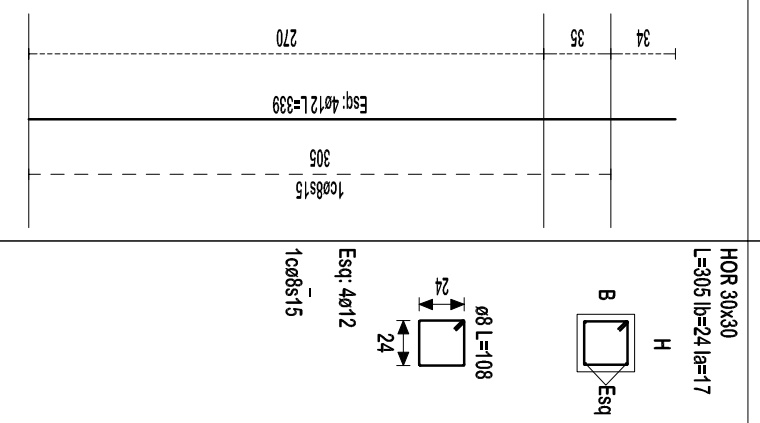



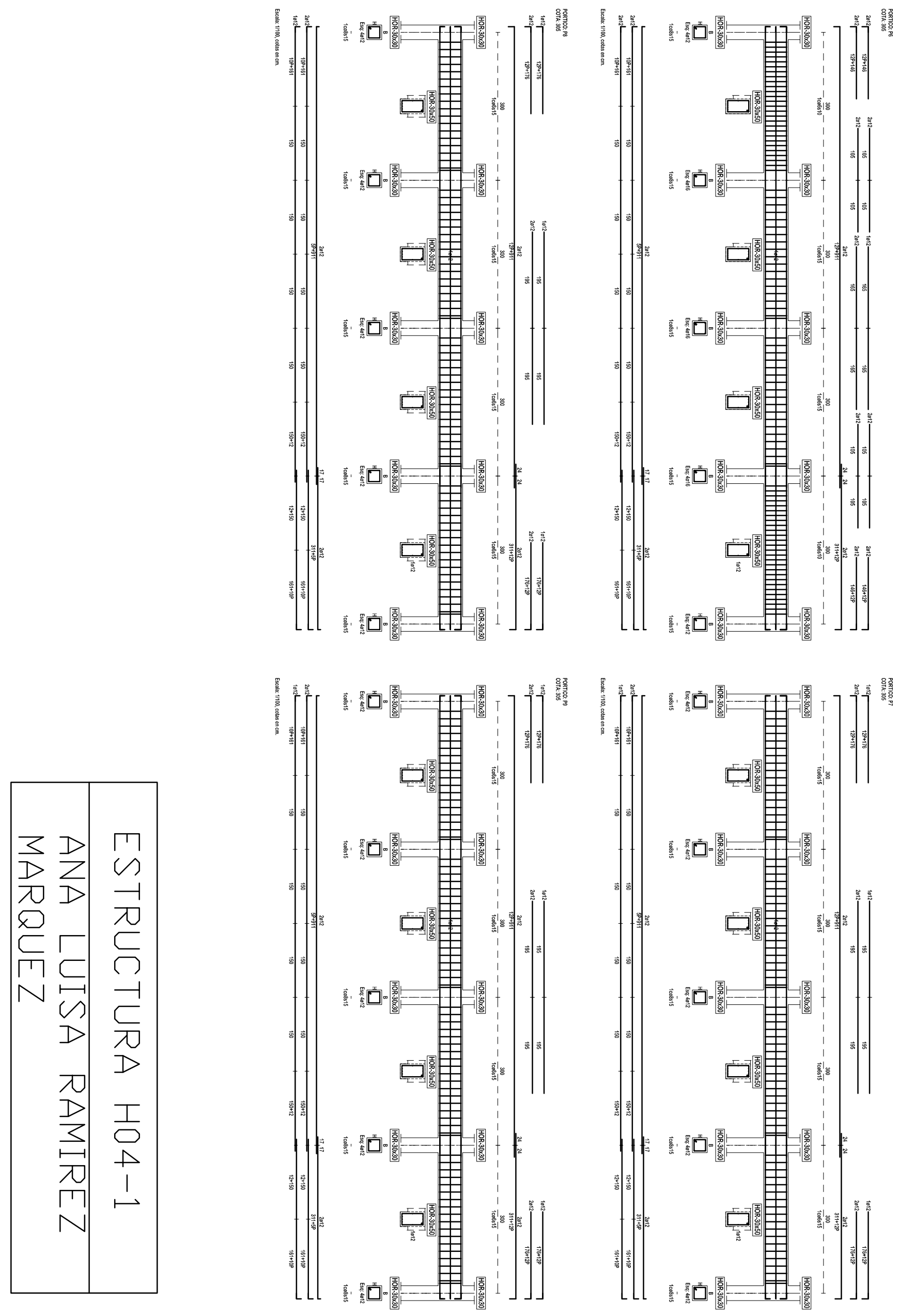

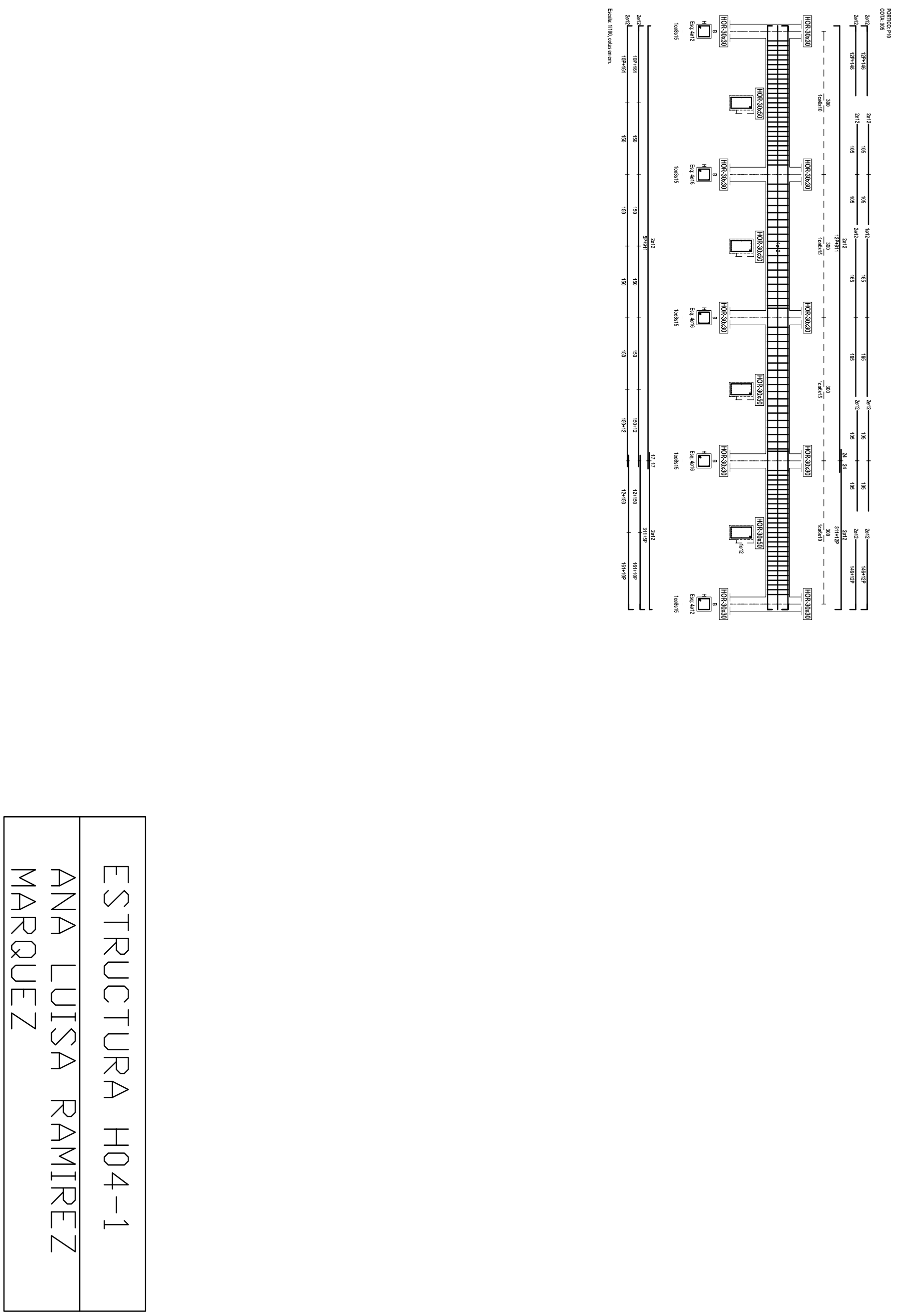

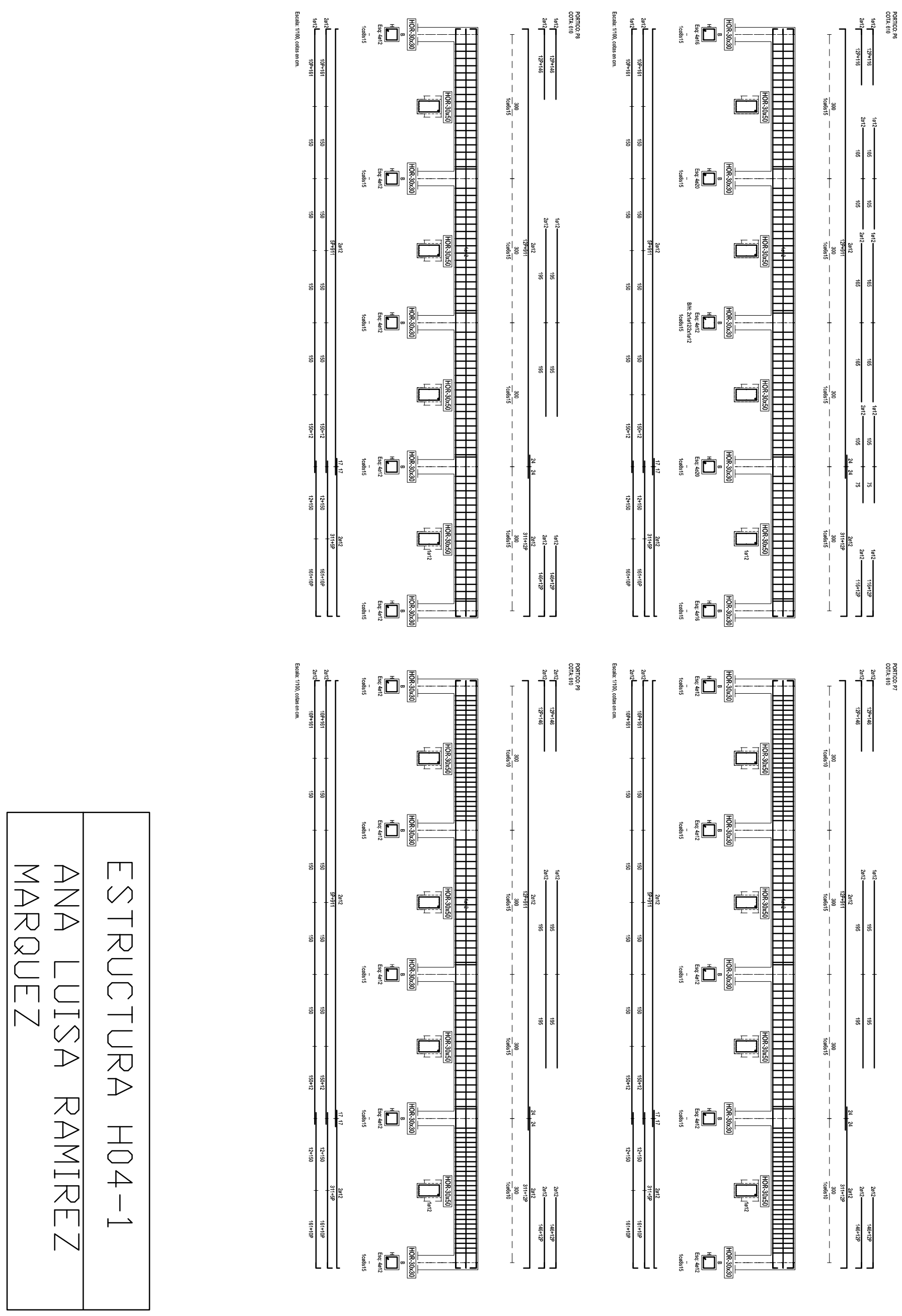

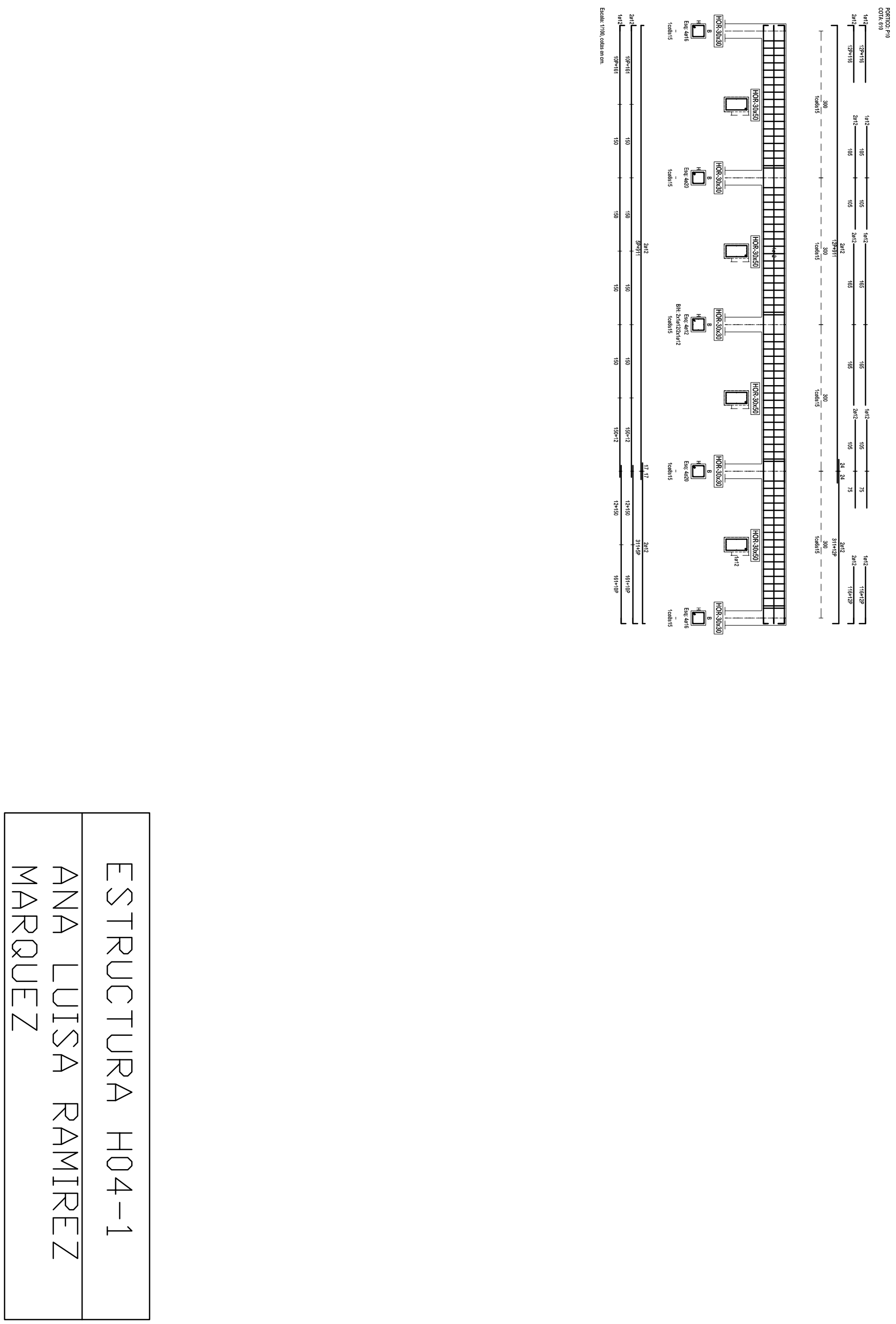

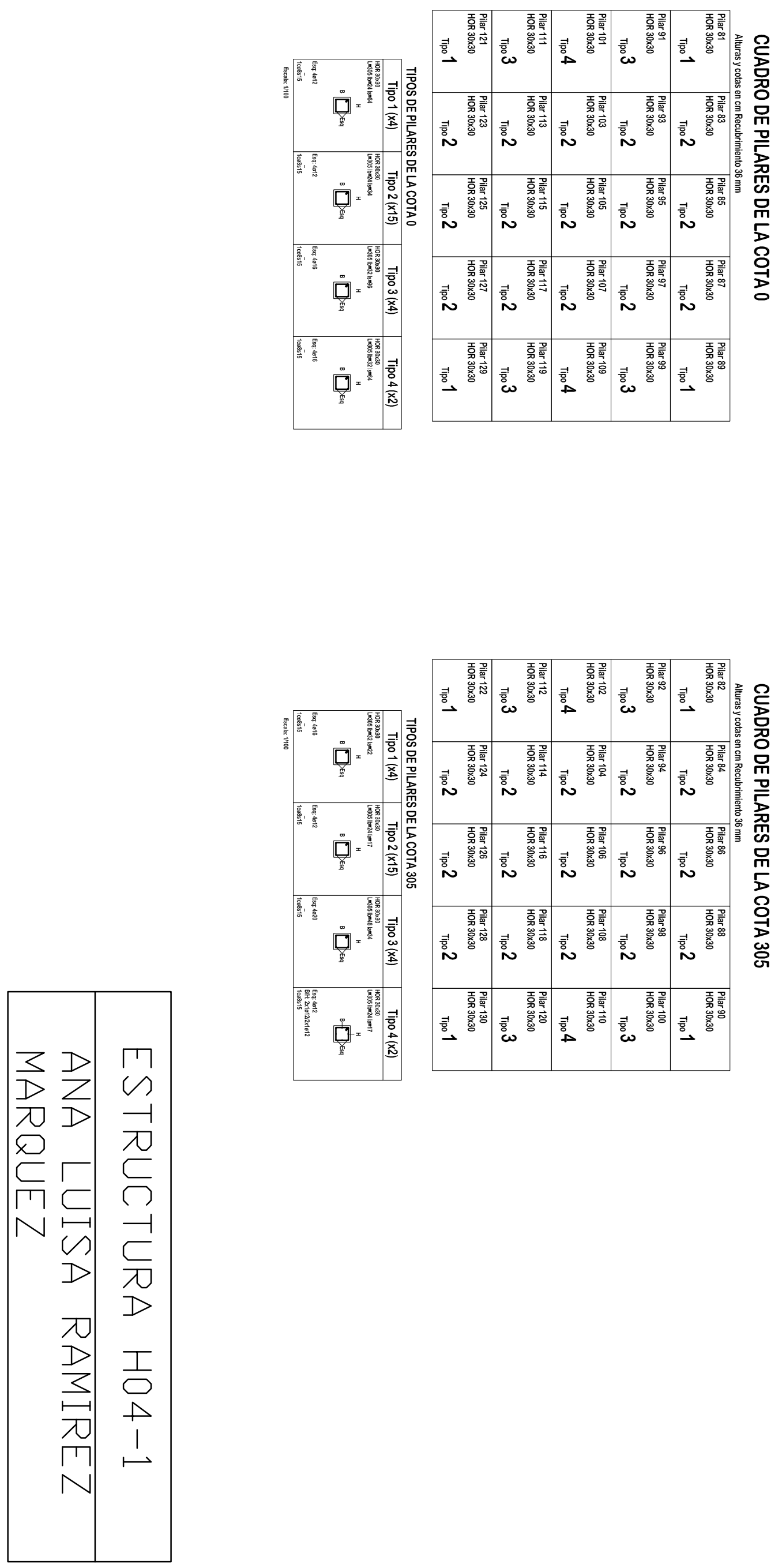


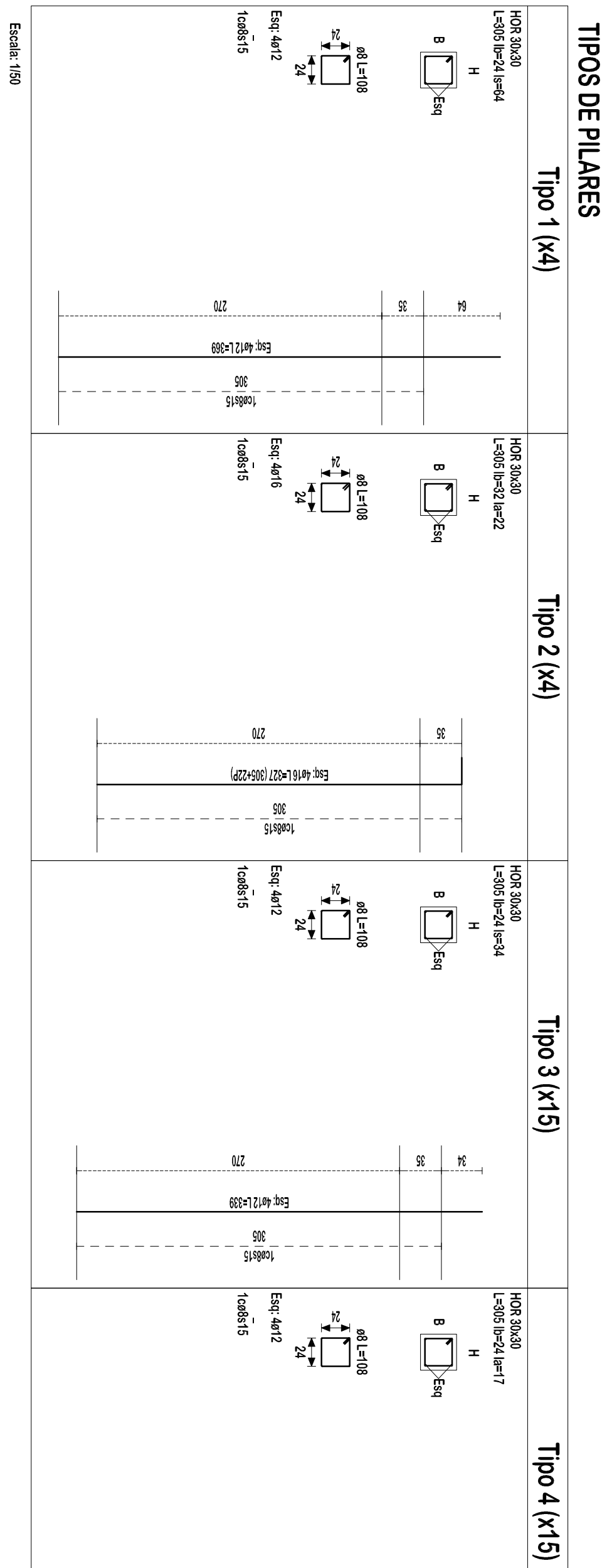



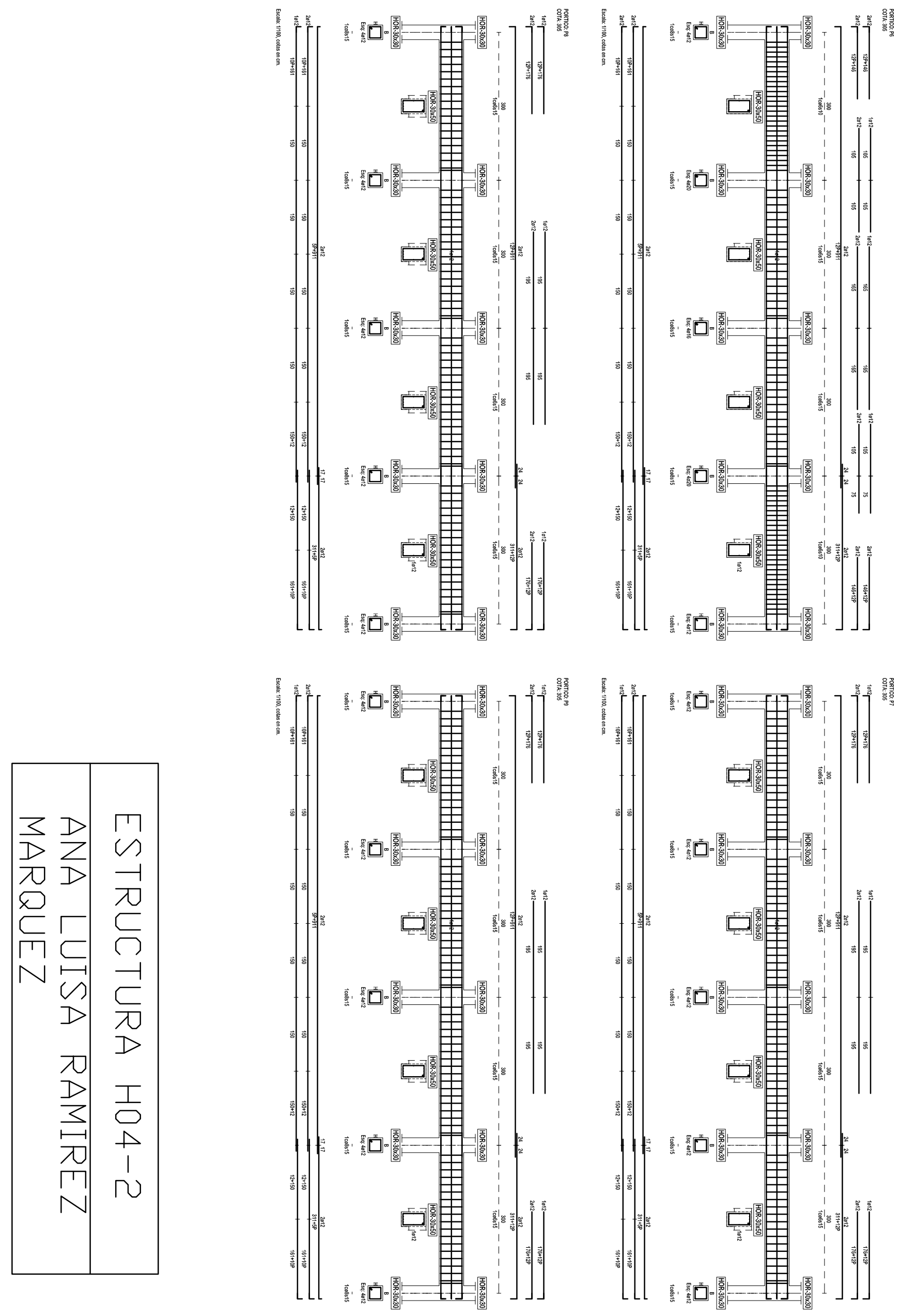


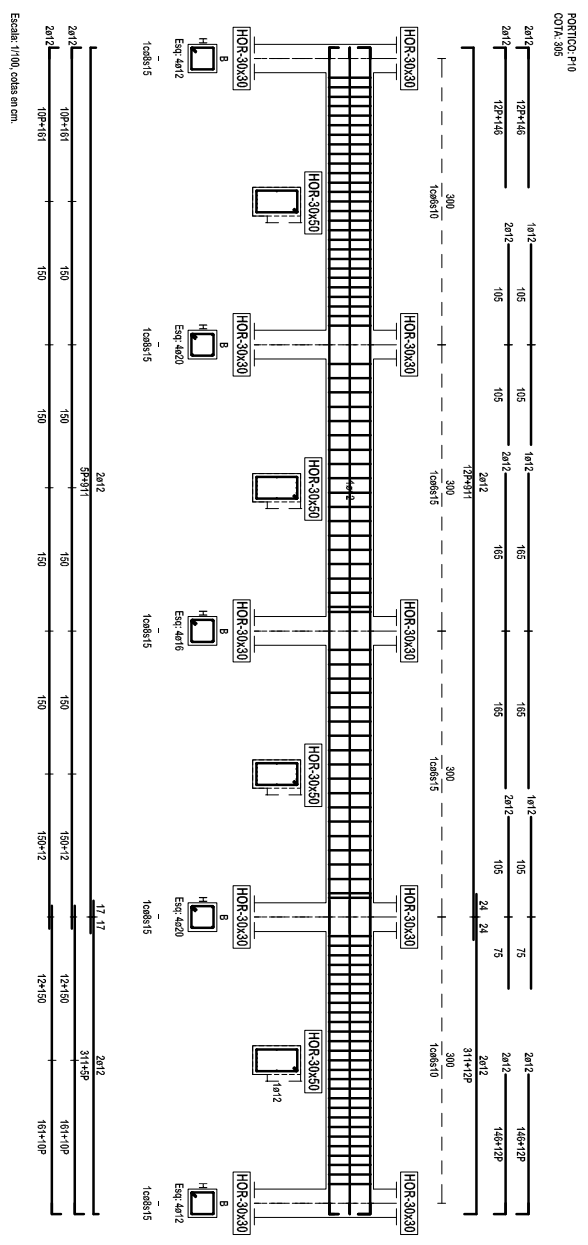

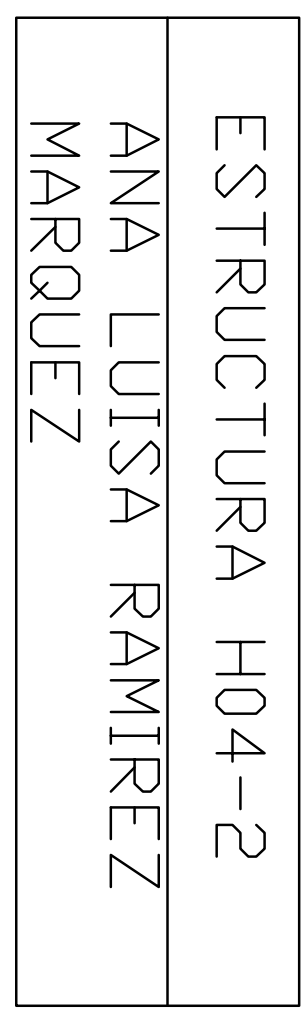



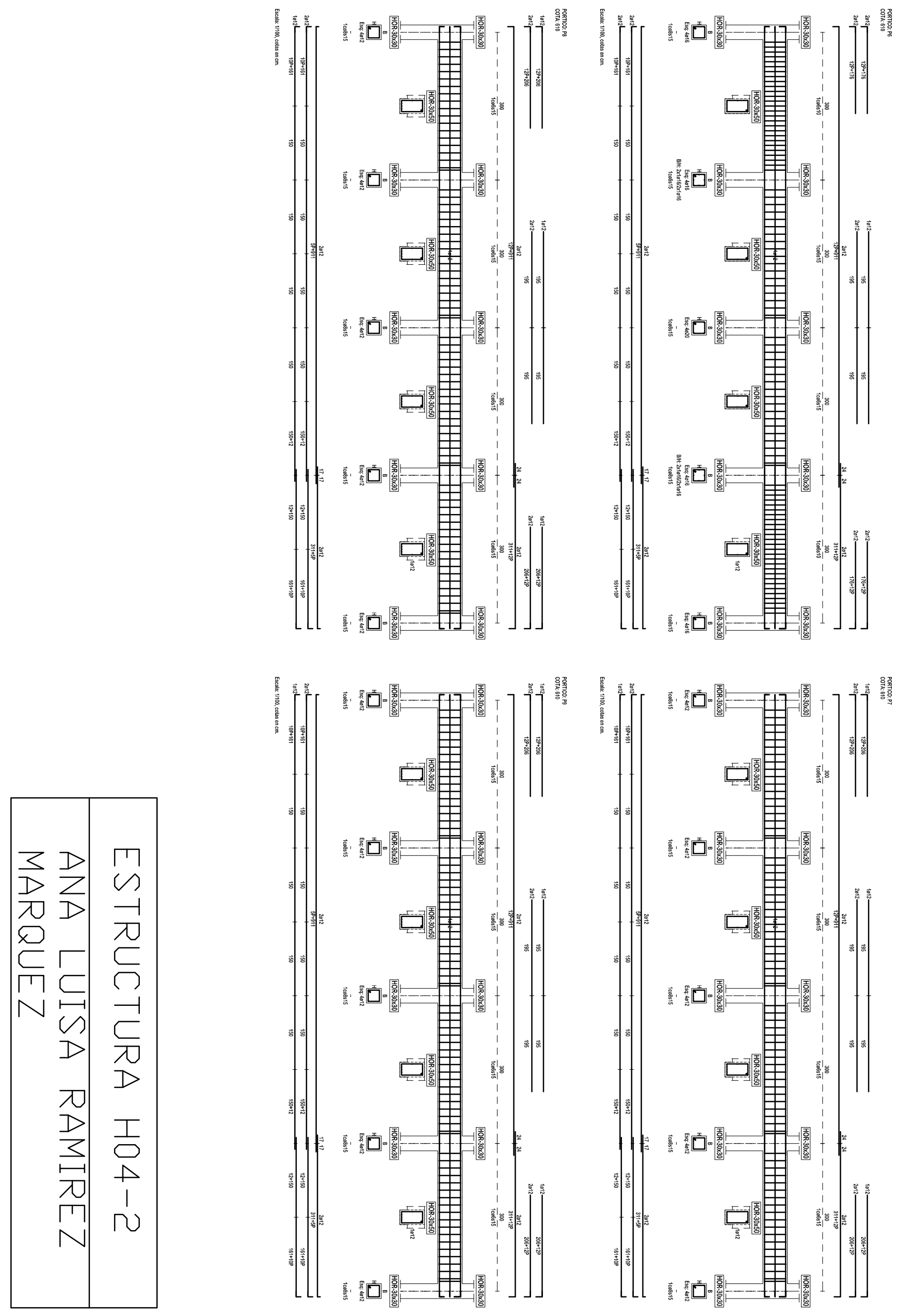

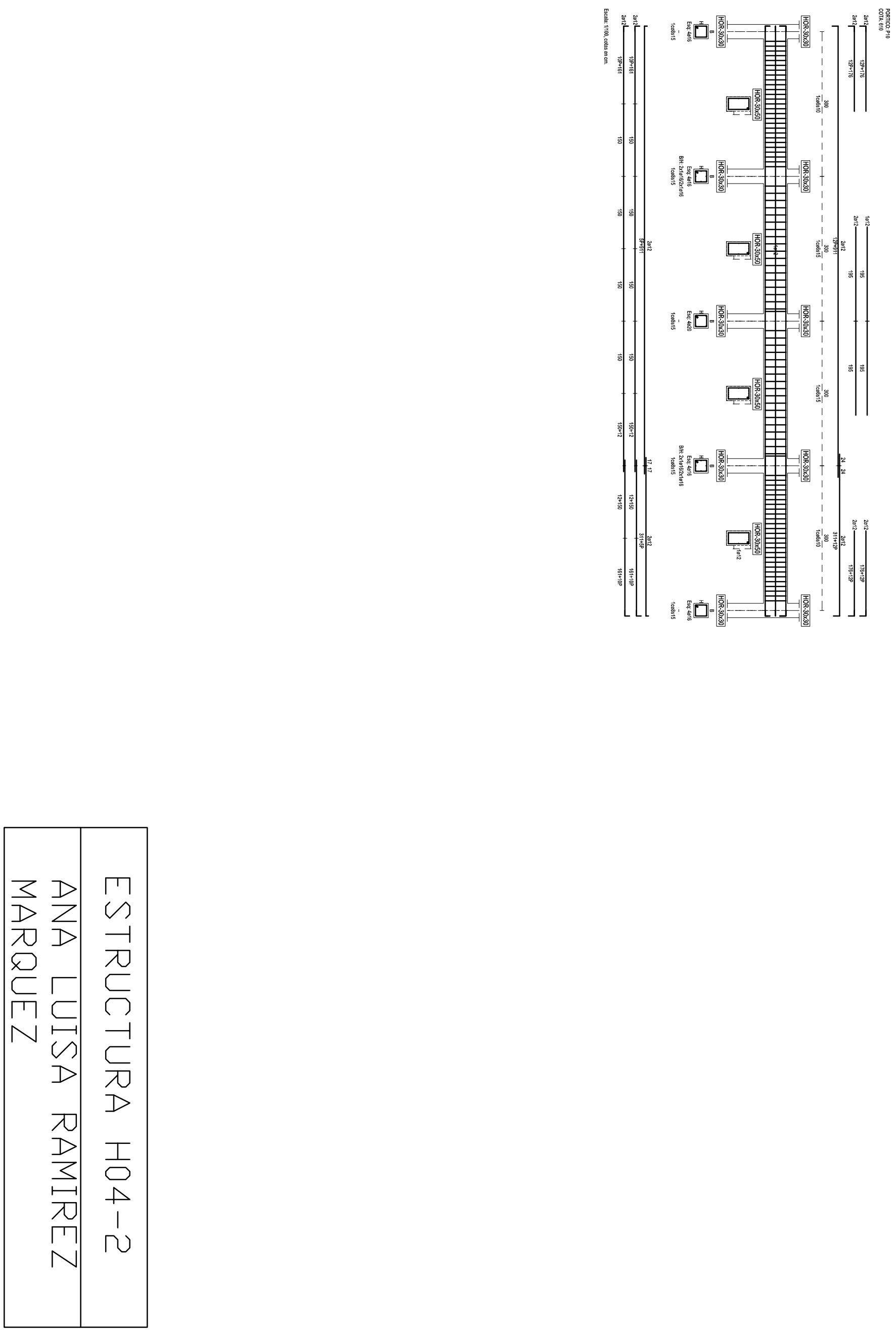

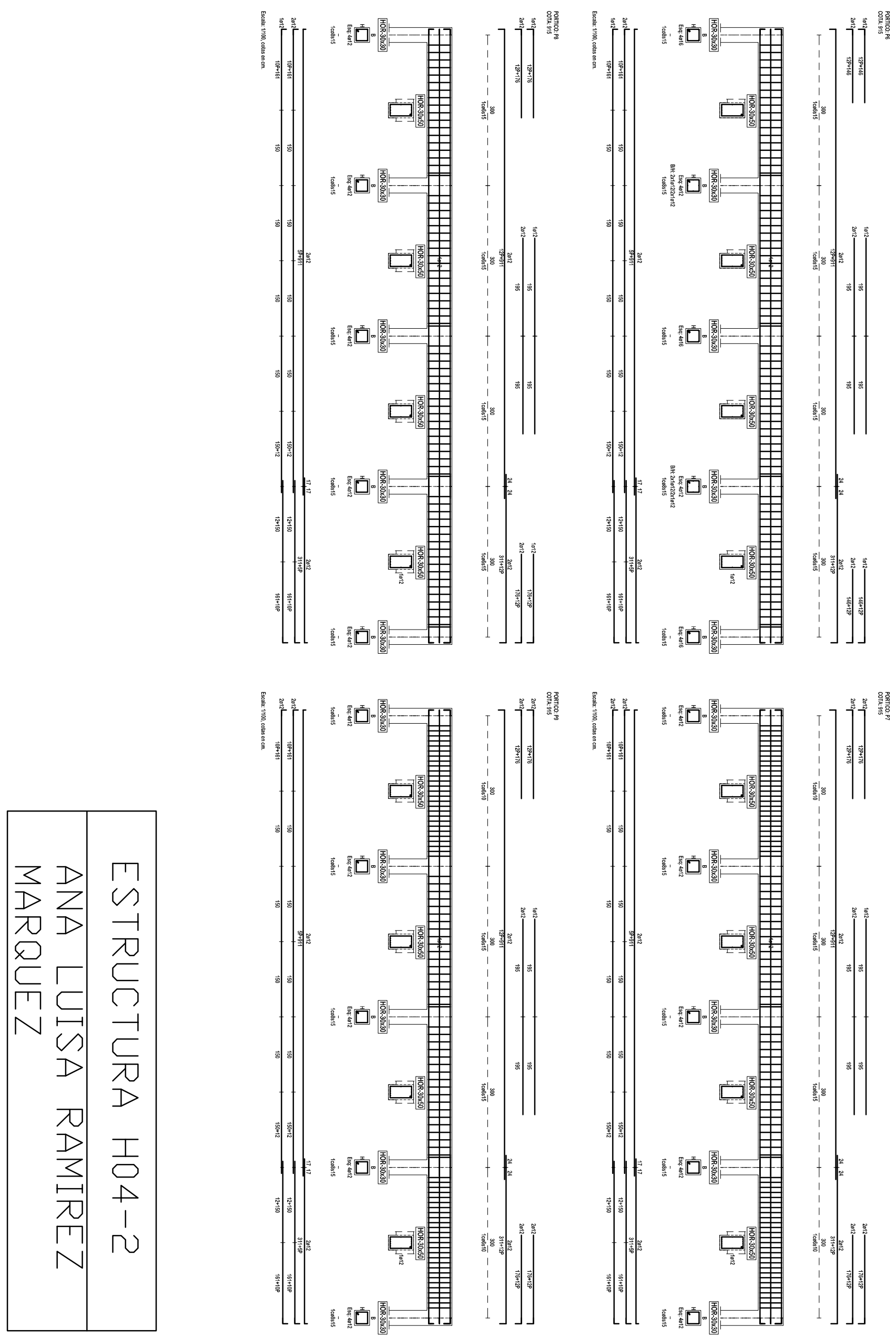


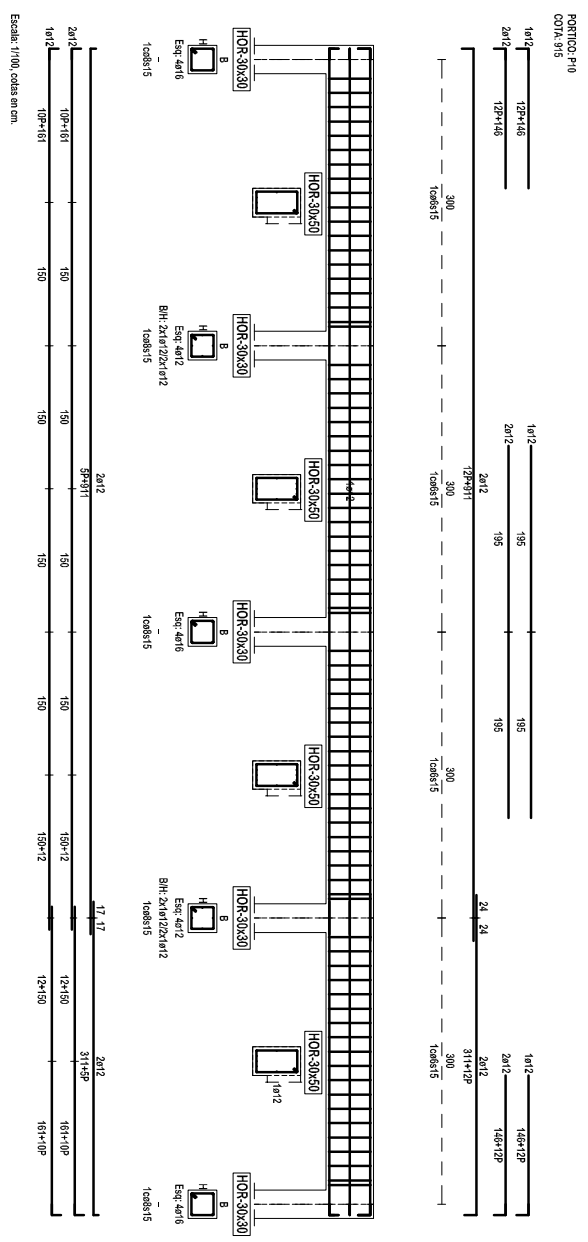

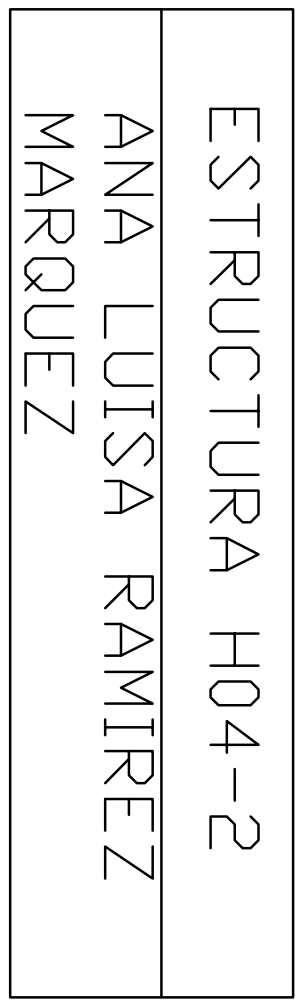



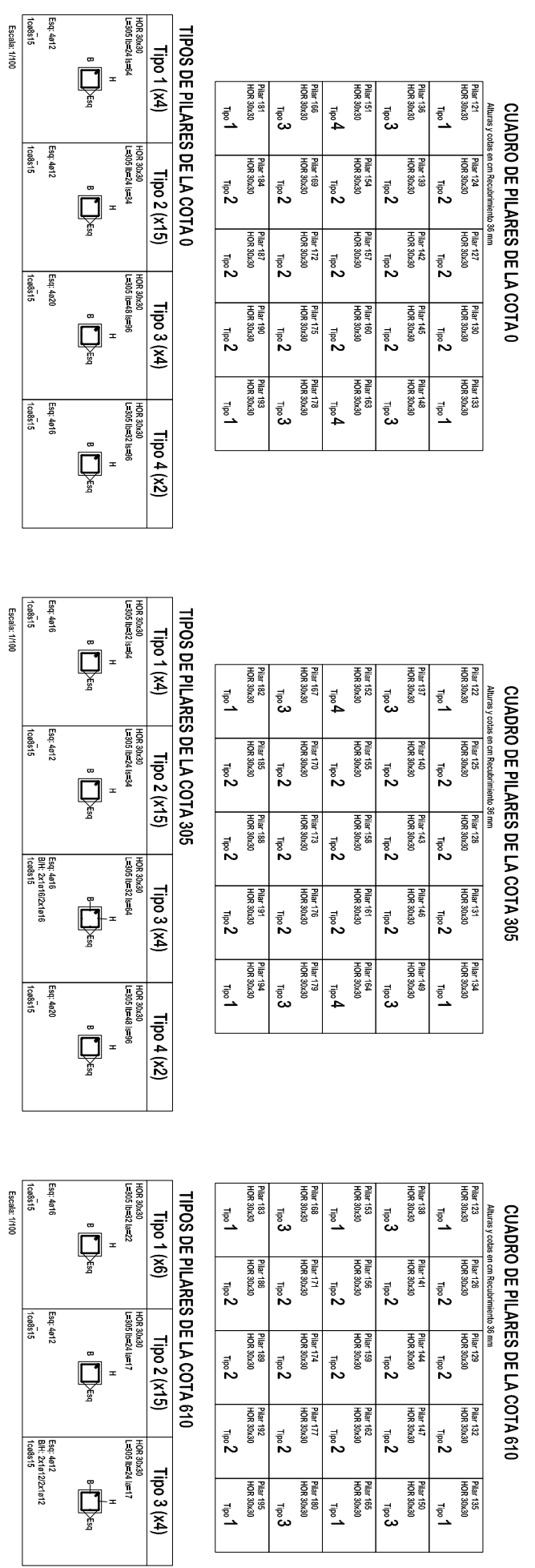